\title{
A FLEXIBLE CONSTRUCTION OF EQUIVARIANT FLOER HOMOLOGY AND APPLICATIONS
}

\author{
KRISTEN HENDRICKS, ROBERT LIPSHITZ, AND SUCHARIT SARKAR
}

\begin{abstract}
Seidel-Smith and Hendricks used equivariant Floer cohomology to define some spectral sequences from symplectic Khovanov homology and Heegaard Floer homology. These spectral sequences give rise to Smith-type inequalities. Similar-looking spectral sequences have been defined by Lee, Bar-Natan, Ozsváth-Szabó, Lipshitz-Treumann, Szabó, Sarkar-Seed-Szabó, and others. In this paper we give another construction of equivariant Floer cohomology with respect to a finite group action and use it to prove some invariance properties of these spectral sequences; prove that some of these spectral sequences agree; improve Hendricks's Smith-type inequalities; give some theoretical and practical computability results for these spectral sequences; define some new spectral sequences conjecturally related to Sarkar-Seed-Szabó's; and introduce a new concordance homomorphism and concordance invariants. We also digress to prove invariance of Manolescu's reduced symplectic Khovanov homology.
\end{abstract}

\section{Contents}

1. Introduction 2

2. Background on equivariant cohomology 8

3. The equivariant Floer complex via non-equivariant complex structures $\quad 10$

3.1. Hypotheses and statement of result 10

3.2. The extended space of eventually cylindrical almost complex structures 11

3.3. Homotopy coherent diagrams of almost complex structures 13

$\begin{array}{ll}\text { 3.4. Building the equivariant cochain complex } & 19\end{array}$

3.5. Invariance 22

3.6. Non-transverse intersections 26

3.7. Other groups 27

4. Equivariant Floer homology via equivariant complex structures 28

4.1. The case of involutions 28

4.2. General finite groups 30

5. Spectral sequences for periodic knots and branched double covers 31

5.1. First invariance statements 31

5.2. Computing spectral sequences from equivariant diagrams 33

5.3. Equivariantly destabilizing basepoints and invariance of the spectral sequences 34

5.4. Equivalence of the Hendricks and Lipshitz-Treumann spectral sequences 38

5.5. Invariants of covering spaces 40

6. New spectral sequences from the branched double cover 41

Date: April 18, 2017.

KH was supported by NSF grant DMS-1506358.

RL was supported by NSF grant DMS- 1149800 .

SS was supported by NSF grant DMS-1350037. 
6.1. A concordance homomorphism from the $\mathbb{Z} / 2$-action on $\widehat{C F}(\Sigma(K)) \quad 41$

6.2. Many concordance invariants from the $\mathbb{Z} / 2$-action on $C F^{-}(\Sigma(K)) \quad 53$

6.3. Computations $\quad 57$

7. Spectral sequences from symplectic Khovanov homology 63

7.1. A brief review of symplectic Khovanov homology 64

7.2. A brief review of cyclic and dihedral groups $\quad 68$

7.3. A localization result $\quad 71$

7.4. Equivariant invariance $\quad 72$

7.5. Invariance of reduced symplectic Khovanov homology 79

7.6. Some conjectures $\quad 81$

Appendix A. Instructive Examples $\quad 83$

$\begin{array}{ll}\text { References } & 87\end{array}$

\section{INTRODUCTION}

Given a space $X$ with a $\mathbb{Z} / 2$-action $\tau: X \rightarrow X$, one can form the Borel equivariant cohomology $H_{\mathbb{Z} / 2}^{*}(X)=H^{*}\left(X \times_{\mathbb{Z} / 2} E \mathbb{Z} / 2\right)$. (Here and throughout, we always work over the field $\mathbb{F}_{2}$ with two elements.) The Serre spectral sequence for the fibration $X \rightarrow X \times_{\mathbb{Z} / 2}$ $E \mathbb{Z} / 2 \rightarrow B \mathbb{Z} / 2$ and the fact that the cellular chain complex of $B \mathbb{Z} / 2=\mathbb{R} P^{\infty}$ satisfies $C^{*}\left(B \mathbb{Z} / 2 ; \mathbb{F}_{2}\right) \cong \mathbb{F}_{2}[\theta]$ combine to give a spectral sequence $H^{*}(X) \otimes \mathbb{F}_{2}[\theta] \Rightarrow H_{\mathbb{Z} / 2}^{*}(X)$ which is, transparently, an invariant of the pair $(X, \tau)$. The $E_{2}$-page is the group cohomology of $\mathbb{Z} / 2$ with coefficients in $H^{*}(X), H^{*}\left(\mathbb{Z} / 2, H^{*}(X)\right)$. If $X$ is a finite CW complex then Smith theory relates $H_{\mathbb{Z} / 2}^{*}(X)$ with the homology of the fixed set $X^{f i x}$ of $\tau$.

Given a chain complex $C_{*}$ with a $\mathbb{Z} / 2$ action, there is a purely algebraic construction of the equivariant cohomology $H_{\mathbb{Z} / 2}^{*}\left(C_{*}\right)$, which is a module over $\mathbb{F}_{2}[\theta]$, and of the spectral sequence from $H^{*}\left(C_{*}\right) \otimes \mathbb{F}_{2}[\theta]$ to $H_{\mathbb{Z} / 2}^{*}\left(C_{*}\right)$, with $E_{2}$-page equal to $H^{*}\left(\mathbb{Z} / 2, H^{*}\left(C_{*}\right)\right)$; see Section 2 for a brief review of this homological algebra. Further, up to isomorphism, $H_{\mathbb{Z} / 2}^{*}\left(C_{*}\right)$, as an $\mathbb{F}_{2}[\theta]$-module, and every page of the spectral sequence $H^{*}\left(C_{*}\right) \otimes \mathbb{F}_{2}[\theta] \Rightarrow H_{\mathbb{Z} / 2}^{*}\left(C_{*}\right)$ depends only on the quasi-isomorphism type of $C_{*}$ as a chain complex over the group ring $\mathbb{F}_{2}[\mathbb{Z} / 2]$.

Seidel-Smith gave an analogue of this story in Lagrangian intersection Floer homology. In particular, given a symplectic manifold $(M, \omega)$, a pair of Lagrangians $L_{0}, L_{1} \subset M$, and a symplectic involution $\tau: M \rightarrow M$ satisfying certain geometric assumptions, they construct a Floer complex $C F\left(L_{0}, L_{1}\right)$ with an action of $\mathbb{Z} / 2$, and from it an equivariant Floer cohomology $H F_{\mathbb{Z} / 2}\left(L_{0}, L_{1}\right)$ and a spectral sequence $H F^{*}\left(L_{0}, L_{1}\right) \otimes \mathbb{F}_{2}[\theta] \Rightarrow H F_{\mathbb{Z} / 2}\left(L_{0}, L_{1}\right)$. Again, the $E_{2}$-page is the group cohomology $H^{*}\left(\mathbb{Z} / 2, H F\left(L_{0}, L_{1}\right)\right)$ of $\mathbb{Z} / 2$ with coefficients in $H F^{*}\left(L_{0}, L_{1}\right){ }^{1}$ More remarkably, they relate $H F_{\mathbb{Z} / 2}\left(L_{0}, L_{1}\right)$ to the Floer cohomology of the fixed parts of $L_{0}$ and $L_{1}$, in the fixed set of $M$.

Seidel-Smith's construction starts by choosing a large Hamiltonian isotopy of $L_{0}$ and $L_{1}$ with certain properties. In particular, it is not immediately obvious that $H F_{\mathbb{Z} / 2}\left(L_{0}, L_{1}\right)$ is an invariant of the equivariant isotopy class of $L_{0}$ and $L_{1}$. Proving invariance is the first goal of this paper:

\footnotetext{
${ }^{1}$ We will use $H F$ to denote Floer homology, $H F^{*}$ to denote Floer cohomology, and $H F_{\mathbb{Z} / 2}$ to denote equivariant Floer cohomology.
} 
Theorem 1.1. The quasi-isomorphism type of the chain complex $C F\left(L_{0}, L_{1}\right)$ over $\mathbb{F}_{2}[\mathbb{Z} / 2]$ is an invariant of $\left(L_{0}, L_{1}\right)$ up to Hamiltonian isotopy through $\mathbb{Z} / 2$-equivariant Lagrangians. In particular, the equivariant Floer cohomology $H F_{\mathbb{Z} / 2}\left(L_{0}, L_{1}\right)$ and the spectral sequence $H F^{*}\left(L_{0}, L_{1}\right) \otimes \mathbb{F}_{2}[\theta] \Rightarrow H F_{\mathbb{Z} / 2}\left(L_{0}, L_{1}\right)$ are invariants of the equivariant Hamiltonian isotopy type of $\left(L_{0}, L_{1}\right)$.

In fact, the equivariant Floer cohomology is often invariant under even non-equivariant isotopies; see Proposition 3.25.

The techniques involved in the proof of Theorem 1.1 are suggested by Seidel-Smith's discussion of Morse theory [SS10, Section 2(a)], and the result would presumably have appeared in Seidel-Smith's paper had they a use for it. (Indeed, some version seems implicit in the discussion around [SS10, Formula (97)].) Seidel-Smith's definition of $H F_{\mathbb{Z} / 2}\left(L_{0}, L_{1}\right)$ requires equivariant transversality, which is guaranteed only after the large Hamiltonian isotopy. The strategy in proving Theorem 1.1 is to define $H F_{\mathbb{Z} / 2}\left(L_{0}, L_{1}\right)$ without using equivariant complex structures, making transversality easier to achieve. This construction has a simple extension to any finite group, though we continue to work with coefficients in $\mathbb{F}_{2}$ to avoid discussing orientations of moduli spaces; see Section 3.7. (Since we are working with coefficients in $\mathbb{F}_{2}$, the construction depends only on the 2-subgroups of the finite group, and is therefore not interesting for odd-order groups.)

Seidel-Smith apply their construction to their symplectic Khovanov homology, to obtain a spectral sequence

$$
K h_{\text {symp }}(K) \otimes \mathbb{F}_{2}\left[\theta, \theta^{-1}\right] \Rightarrow \widehat{H F}_{\nabla}^{*}(\Sigma(K)) \otimes \mathbb{F}_{2}\left[\theta, \theta^{-1}\right]
$$

from Seidel-Smith's symplectic Khovanov homology of $K$ [SS06] to a variant of the Heegaard Floer cohomology of the branched double cover of the mirror of $K$. (Seidel-Smith denote $\widehat{H F}_{\nabla}^{*}$ by $K h_{\text {symp, inv }}(K)$; we choose the notation $\widehat{H F}_{\nabla}^{*}(\Sigma(K))$ because Seidel-Smith conjecture that the invariant is isomorphic to $\widehat{H F}^{*}\left(\Sigma(K) \#\left(S^{2} \times S^{1}\right)\right)$.) Given a 2-periodic knot $\widetilde{K}$ with quotient $K$, they also obtain a spectral sequence

$$
K h_{\text {symp }}(\widetilde{K}) \otimes \mathbb{F}_{2}\left[\theta, \theta^{-1}\right] \Rightarrow K h_{\text {symp }}(K) \otimes \mathbb{F}_{2}\left[\theta, \theta^{-1}\right] .
$$

As a corollary of Theorem 1.1 we obtain:

Corollary 1.4. Every page of Seidel-Smith's spectral sequence (1.2) is an invariant of $K$.

A version of Corollary 1.4 was observed by Seidel-Smith [SS10, p. 1496], though they do not spell out the details of the argument. Note that we are not asserting that the isomorphism between the $E_{\infty}$-page of the sequence (1.2) and $\widehat{H F}_{\nabla}^{*} \otimes \mathbb{F}_{2}\left[\theta, \theta^{-1}\right]$ is itself a knot invariant; see also Seidel-Smith's discussion [SS10, Section 4.4]. The fact that $\widehat{H F}_{\nabla}^{*}(\Sigma(K))$ is an invariant of $K$ was also proved by Tweedy [Twe14]; a sketch of a proof was also given by Seidel-Smith [SS10, Section 4.4]. (In fact, there is a spectral sequence $\widehat{H F}_{\nabla}^{*}(\Sigma(K)) \Rightarrow$ $\widehat{H F}^{*}\left(\Sigma(K) \#\left(S^{2} \times S^{1}\right)\right)[\mathrm{SS} 06$, Section $4 \mathrm{~d}]$, and Tweedy showed that this spectral sequence is a knot invariant.)

Corollary 1.5. Every page of Seidel-Smith's spectral sequence (1.3) is an invariant of the knot $\widetilde{K}$ together with its free period, or equivalently of the pair $(K, A)$ of the knot $K$ and the axis $A$ of the period.

Using Seidel-Smith's localization theorem, Hendricks constructed four spectral sequences in Heegaard Floer cohomology: 
- Given a knot $K$ in $S^{3}$ and a sufficiently large integer $n$, a spectral sequence

$$
\widehat{H F K}^{*}(\Sigma(K), \widetilde{K}) \otimes V^{\otimes n} \otimes \mathbb{F}_{2}\left[\theta, \theta^{-1}\right] \Rightarrow \widehat{H F K}^{*}\left(S^{3}, K\right) \otimes V^{\otimes n} \otimes \mathbb{F}_{2}\left[\theta, \theta^{-1}\right]
$$

from the knot Floer cohomology of the preimage $\widetilde{K}$ of $K$ in its branched double cover to the knot Floer cohomology of $K$ in $S^{3}$ and a spectral sequence

$$
\widehat{H F}^{*}(\Sigma(K)) \otimes V^{\otimes n} \otimes \mathbb{F}_{2}\left[\theta, \theta^{-1}\right] \Rightarrow V^{\otimes n} \otimes \mathbb{F}_{2}\left[\theta, \theta^{-1}\right] .
$$

- Given a 2-periodic knot $\widetilde{K}$ in $S^{3}$ with axis $\widetilde{A}$ whose quotient knot $K$ has axis $A$, and a sufficiently large integer $n$, a spectral sequence

$$
\widehat{H F L}^{*}(\widetilde{K} \cup \widetilde{A}) \otimes V_{1}^{\otimes(2 n-1)} \otimes \mathbb{F}_{2}\left[\theta, \theta^{-1}\right] \Rightarrow \widehat{H F L}^{*}(K \cup A) \otimes V_{1}^{\otimes(n-1)} \otimes \mathbb{F}_{2}\left[\theta, \theta^{-1}\right]
$$

from the link Floer cohomology of $\widetilde{K} \cup A$ to the link Floer cohomology of $K \cup A$, and a spectral sequence

$$
\widehat{H F K}^{*}(\widetilde{K}) \otimes V^{\otimes(2 n-1)} \otimes W \otimes \mathbb{F}_{2}\left[\theta, \theta^{-1}\right] \Rightarrow \widehat{H F K}^{*}(K) \otimes V^{\otimes(n-1)} \otimes W \otimes \mathbb{F}_{2}\left[\theta, \theta^{-1}\right] .
$$

(Here, $V, V_{1}$, and $W$ are 2-dimensional graded vector spaces. In all cases there are similar, albeit more complex, statements for links.) Theorem 1.1 also implies:

Corollary 1.10. Every page of the spectral sequence (1.6) is an invariant of the pair $(K, n)$.

Corollary 1.11. Every page of the spectral sequences (1.8) and (1.9) is an invariant of the integer $n$, the knot $\widetilde{K}$, and the 2-period $\tau:\left(S^{3}, \widetilde{K}\right) \rightarrow\left(S^{3}, \widetilde{K}\right)$ or, equivalently, of the triple $(K, A, n)$.

Typically, direct computations of Floer cohomology take advantage of symmetries of the Lagrangians. A side effect of Seidel-Smith's construction of $H F_{\mathbb{Z} / 2}\left(L_{0}, L_{1}\right)$ is that one tends to lose such symmetries, making computations challenging. The techniques used to prove Theorem 1.1 mean that often one can compute the equivariant Floer cohomology without perturbing the Lagrangians. A precise statement is given in Proposition 4.3; here are some consequences:

Corollary 1.12. Let $K$ be a knot in $S^{3}$ and let $\mathcal{H}=\left(S^{2}, \boldsymbol{\alpha}, \boldsymbol{\beta}, \mathbf{z}, \mathbf{w}\right)$ be a genus-0 Heegaard diagram for $K$ with $|\boldsymbol{\alpha}|=n$. Let $\widetilde{\mathcal{H}}=(\Sigma, \widetilde{\boldsymbol{\alpha}}, \widetilde{\boldsymbol{\beta}}, \widetilde{\mathbf{z}}, \widetilde{\mathbf{w}})$ be the double cover of $\mathcal{H}$ branched along $\mathbf{z} \cup \mathbf{w}$ so that $\widetilde{\mathcal{H}}$ represents $(\Sigma(K), K)$. Then for a generic one-parameter family $\widetilde{J}$ of $\mathbb{Z} / 2$-invariant almost complex structures on $\operatorname{Sym}^{2 n}(\Sigma)$, the spectral sequence (1.6) is induced by the double complex

$$
0 \longrightarrow\left(C F\left(T_{\widetilde{\alpha}}, T_{\widetilde{\beta}}\right), \partial_{\widetilde{J}}\right) \stackrel{\mathbb{I}+\tau^{\#}}{\longrightarrow}\left(C F\left(T_{\widetilde{\alpha}}, T_{\widetilde{\beta}}\right), \partial_{\widetilde{J}}\right) \stackrel{\mathbb{I}+\tau^{\#}}{\longrightarrow} \cdots
$$

An analogous statement holds for the spectral sequence (1.8) and any genus-0 Heegaard diagram $\mathcal{H}$ for $K \cup A$ and the induced diagram for $\widetilde{K} \cup \widetilde{A}$.

Corollary 1.13. For any knot $K$ in $S^{3}$, the spectral sequences (1.6), (1.8), and (1.9) can be computed combinatorially from a genus-0 nice Heegaard diagram.

For the spectral sequences (1.6) and (1.8), any genus-0 nice diagram, such as Beliakova's planar grid diagrams [Bel10], suffices. For the spectral sequence (1.9), we need a specially adapted diagram, like those constructed in Section 5.2.

Using the fact that we can compute the spectral sequences from an equivariant Heegaard diagram, we can also understand the behavior under changing $n$ : 
Theorem 1.14. For any knot $K$ in $S^{3}$ there is a spectral sequence $\widehat{H F K}^{*}(\Sigma(K), \widetilde{K}) \otimes$ $\mathbb{F}_{2}\left[\theta, \theta^{-1}\right] \Rightarrow \widehat{H F K}^{*}\left(S^{3}, K\right) \otimes \mathbb{F}_{2}\left[\theta, \theta^{-1}\right]$ so that the spectral sequence (1.6) is obtained by tensoring with $V^{\otimes n}$.

A consequence of Hendricks' spectral sequence (1.6) was a rank inequality

$$
\operatorname{dim}\left(\widehat{H F K}\left(\Sigma(K), \widetilde{K}, \mathfrak{s}_{0}\right) \geq \operatorname{dim}\left(\widehat{H F K}\left(S^{3}, K\right)\right)\right.
$$

where $\mathfrak{s}_{0}$ is the unique spin structure on $\Sigma(K)$, and the same rank inequality for the restriction of the theory to the extremal Alexander gradings $\pm g(K)$, to wit

$$
\operatorname{dim}\left(\widehat{H F K}\left(\Sigma(K), \widetilde{K}, \mathfrak{s}_{0}, \pm g(K)\right) \geq \operatorname{dim}\left(\widehat{H F K}\left(S^{3}, K, \pm g(K)\right) .\right.\right.
$$

Theorem 1.14 allows us to extract this inequality for all Alexander gradings.

Corollary 1.15. For any knot $K$ in $S^{3}$, let $\mathfrak{s}_{0}$ be the unique spin structure on $\Sigma(K)$ and let $i$ be any Alexander grading. There is a rank inequality

$$
\operatorname{dim}\left(\widehat{H F K}\left(\Sigma(K), \widetilde{K}, \mathfrak{s}_{0}, i\right)\right) \geq \operatorname{dim}\left(\widehat{H F K}\left(S^{3}, K, i\right)\right)
$$

To state the analogue of Theorem 1.14 for the spectral sequences (1.8) and (1.9) we need a little more notation. Let $X=\mathbb{F}_{2}\langle x x, x y, y x, y y\rangle$ with differential $d_{1}(x x)=d_{1}(y y)=0$ and $d_{1}(x y)=d_{1}(y x)=x y+y x$. Let $Y$ be the homology of $X$.

Theorem 1.16. For any 2-periodic link $\widetilde{K}$ in $S^{3}$ with axis $\widetilde{A}$ and quotient link $K$ there is a spectral sequence

$$
\widehat{H F L}^{*}(\widetilde{K} \cup \widetilde{A}) \otimes \mathbb{F}_{2}\left[\theta, \theta^{-1}\right] \otimes V_{1} \Rightarrow \widehat{H F L}^{*}(K \cup A) \otimes \mathbb{F}_{2}\left[\theta, \theta^{-1}\right]
$$

so that the spectral sequence (1.8) is obtained by tensoring with $X^{\otimes n-1}$. That is, let $E_{i}(K)$ be the spectral sequence (1.8) and let $\widetilde{E}_{i}(K)$ be the spectral sequence (1.17). Then:

- $E_{1}(K) \cong \widetilde{E}_{1}(K) \otimes X^{\otimes n-1}$, where the differential on the right hand side is the tensor product differential.

- For $i>1, E_{i}(K) \cong \widetilde{E}_{i}(K) \otimes Y^{\otimes n-1}$, where the differential on the right hand side is induced by the differential on $\widetilde{E}_{i}(K)$.

Similarly, there is a spectral sequence

$$
\widehat{H F K}^{*}(\widetilde{K}) \otimes V \otimes W \otimes \mathbb{F}_{2}\left[\theta, \theta^{-1}\right] \Rightarrow \widehat{H F K}^{*}(K) \otimes W \otimes \mathbb{F}_{2}\left[\theta, \theta^{-1}\right]
$$

so that the spectral sequence (1.9) is obtained by tensoring with $X^{\otimes n-1}$.

(There is a version of Theorem 1.16 for links, but the notation is more cumbersome, so we omit it.) Using the spectral sequence (1.17), we prove the following corollary.

Corollary 1.19. For any 2-periodic link $\widetilde{K}$ in $S^{3}$ with axis $\widetilde{A}$ and quotient link $K$, there is a rank inequality

$$
\operatorname{dim}\left(\widehat{H F L}\left(S^{3}, \widetilde{K} \cup \widetilde{A}\right)\right) \geq \operatorname{dim}\left(\widehat{H F L}\left(S^{3}, K \cup A\right)\right)
$$

Notice that there is no factor of two in this rank inequality, despite the presence of the vector space $V_{1}$ in Formula (1.17).

Using Hochschild homology and bordered Floer homology, Lipshitz-Treumann also constructed spectral sequences similar to Formulas (1.6) and (1.8) [LT16]. In particular, for a genus $\leq 2$, null-homologous knot $K \subset Y^{3}$, they give a spectral sequence $\widehat{H F K}(\Sigma(K), K) \otimes$ 
$\mathbb{F}_{2}\left[\theta, \theta^{-1}\right] \Rightarrow \widehat{H F K}(Y, K) \otimes \mathbb{F}_{2}\left[\theta, \theta^{-1}\right][\mathrm{LT} 16$, Theorem 2]. In the case that the Hendricks and Lipshitz-Treumann spectral sequences are both defined, we prove they agree:

Theorem 1.20. Let $K$ be a genus $\leq 2$ knot in $S^{3}$. Then the spectral sequence from Theorem 1.14 agrees with the spectral sequence from [LT16, Theorem 2].

Remark 1.21. To be more precise, [LT16, Theorem 2] worked with equivariant Floer homology, not cohomology, so the identification is between Theorem 1.14 for $(Y, K)$ and [LT16, Theorem 2] for $(-Y, K)$. In this paper, when we refer to complexes from [LT16], we mean the duals of the complexes presented there. The paper [LT16] typically focuses on the Tate version of equivariant cohomology, but here we mean the Borel variant.

It also follows from the techniques in this paper that the spectral sequences constructed by Lipshitz-Treumann are invariants of the corresponding topological objects, and that equivariant Floer cohomology can be computed using bordered Floer homology. Rather than further trying the reader's patience, we leave most cases as an exercise, and spell out only one:

Theorem 1.22. For any $\mathbb{Z} / 2$-cover $\pi: \widetilde{Y} \rightarrow Y$ and torsion $\operatorname{spin}^{c}$-structure $\mathfrak{s}$ on $Y$, there is an equivariant Heegaard Floer homology $\widehat{H F}_{\mathbb{Z} / 2}\left(\widetilde{Y}, \pi^{*}(\mathfrak{s})\right)$ and a spectral sequence $\widehat{H F}^{*}\left(\tilde{Y}, \pi^{*}(\mathfrak{s})\right) \otimes$ $V \otimes \mathbb{F}_{2}[\theta] \Rightarrow \widehat{H F}_{\mathbb{Z} / 2}\left(\widetilde{Y}, \pi^{*}(\mathfrak{s})\right)$ which are invariants of the pair $(\pi: \widetilde{Y} \rightarrow Y, \mathfrak{s})$. This spectral sequence can be computed combinatorially using nice diagrams. If $\widetilde{Y} \rightarrow Y$ is induced by a $\mathbb{Z}$-cover then the equivariant Floer cohomology and the spectral sequence can be computed combinatorially using bordered Floer homology.

A $\mathbb{Z} / 2$ cover is induced by a $\mathbb{Z}$-cover if there is a pullback square

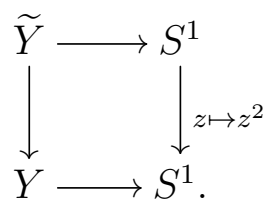

If $\widetilde{Y} \rightarrow Y$ is a $\mathbb{Z} / 2$-cover induced by a $\mathbb{Z}$-cover and $\mathfrak{s}$ is a torsion spin ${ }^{c}$-structure on $Y$ then the localized equivariant Floer cohomology $\theta^{-1} \widehat{H F}_{\mathbb{Z} / 2}\left(\widetilde{Y}, \pi^{*} \mathfrak{s}\right)$ is isomorphic to $\widehat{H F}^{*}(Y, \mathfrak{s}) \otimes$ $\mathbb{F}_{2}\left[\theta, \theta^{-1}\right]\left[\right.$ LT16, Theorem 3]. The restriction to torsion $\operatorname{spin}^{c}$-structures is to ensure that the relevant objects are $\mathbb{Z}$-graded.

(In [LT16], the localized equivariant cohomology is referred to as the Tate equivariant cohomology. The statement of [LT16, Theorem 3] combines the spectral sequence $\widehat{H F}^{*}\left(\widetilde{Y}, \pi^{*} \mathfrak{s}\right) \otimes$ $V \otimes \mathbb{F}_{2}\left[\theta, \theta^{-1}\right] \Rightarrow \theta^{-1} \widehat{H F}_{\mathbb{Z} / 2}^{*}\left(\widetilde{Y}, \pi^{*} \mathfrak{s}\right)$, which exists simply because $\widehat{C F}\left(\widetilde{Y}, \pi^{*} \mathfrak{s}\right) \otimes V$ is a module over $\mathbb{F}_{2}[\mathbb{Z} / 2]$, and the more subtle localization isomorphism $\theta^{-1} \widehat{H F}_{\mathbb{Z} / 2}^{*}\left(\widetilde{Y}, \pi^{*} \mathfrak{s}\right) \cong \widehat{H F}^{*}(Y, \mathfrak{s}) \otimes$ $\mathbb{F}_{2}\left[\theta, \theta^{-1}\right]$, to state a spectral sequence $\widehat{H F}^{*}\left(\widetilde{Y}, \pi^{*} \mathfrak{s}\right) \otimes V \otimes \mathbb{F}_{2}\left[\theta, \theta^{-1}\right] \Rightarrow \widehat{H F}^{*}(Y, \mathfrak{s}) \otimes \mathbb{F}_{2}\left[\theta, \theta^{-1}\right]$.)

Theorem 1.22 extends to finite covering spaces; see Theorem 5.5.

Finally, we discuss two spectral sequences which were partial inspiration for work of SarkarSeed-Szabó [SSS14]. First, in Section 6.1 we observe that applying Seidel-Smith's localization theorem to the involution on the branched double cover $\Sigma(L)$ of a based link $(L, p)$ in $S^{3}$ gives a spectral sequence

$$
\widehat{H F}^{*}(\Sigma(L)) \otimes \mathbb{F}_{2}\left[\theta, \theta^{-1}\right] \Rightarrow\left(\mathbb{F}_{2} \oplus \mathbb{F}_{2}\right)^{\otimes(|L|-1)} \otimes \mathbb{F}_{2}\left[\theta, \theta^{-1}\right] .
$$


Theorem 1.24. The spectral sequence (1.23) is an invariant of the based link $(L, p)$.

From this spectral sequence, we extract a numerical invariant $q_{\tau}(K) \in \mathbb{Z}$, and prove:

Theorem 1.25. The invariant $q_{\tau}(K)$ is a homomorphism from the smooth concordance group.

There are analogous sequences for $H F^{-}(\Sigma(K)$ ) (Theorem 6.13), from which we extract a sequence of integer-valued concordance invariants $d_{\tau}(K, i), i>0 \in \mathbb{Z}$. The invariant $d_{\tau}(K, 1)$ is the $d$-invariant of the spin structure on $\Sigma(K)$, denoted $2 \delta(K)$ by Manolescu-Owens [MO07]. The invariants $d_{\tau}$ satisfy

$$
d_{\tau}(K, 1) \leq d_{\tau}(K, 2) \leq d_{\tau}(K, 3) \leq \cdots
$$

Explicit computations (Section 6.3) show that the invariants $q_{\tau}$ and $d_{\tau}(K, 2)$ do not agree with previously defined concordance invariants.

In the setting of symplectic Khovanov homology, there is an action of the dihedral group $D_{2^{m}}$ for any $m$; see Section 7 . The techniques of Section 3 give a chain complex $\mathcal{C}_{K h}^{\text {symp free }}(L)$, quasi-isomorphic to the symplectic Khovanov cochain complex and is a module over the group ring $\mathbb{F}_{2}\left[D_{2^{m}}\right]$.

Theorem 1.26. The quasi-isomorphism type of $\mathcal{C}_{K h}^{\text {symp free }}(L)$ over $\mathbb{F}_{2}\left[D_{2^{m}}\right]$ is an invariant of the link $L$.

Theorem 1.26 gives several spectral sequences from $\mathcal{C}_{K h \text {,symp }}(L)$. Restricting attention to the $\mathbb{Z} / 2$ subgroup of $D_{2^{m}}$ corresponding to a (particular) reflection gives Seidel-Smith's spectral sequence (1.2). Restricting attention to the cyclic group $\mathbb{Z} / 2 \subset D_{2^{m}}$ generated by rotation by $\pi$ gives a spectral sequence

$$
K h_{\text {symp }}(L) \otimes \mathbb{F}_{2}\left[\theta, \theta^{-1}\right] \Rightarrow\left(\mathbb{F}_{2} \oplus \mathbb{F}_{2}\right)^{\otimes|L|} \otimes \mathbb{F}_{2}\left[\theta, \theta^{-1}\right]
$$

while restricting to the cyclic group $\mathbb{Z} / 2^{m}, m>1$, gives a spectral sequence

$$
K h_{\text {symp }}(L) \otimes \mathbb{F}_{2}\left[\eta, \eta^{-1}\right] \Rightarrow\left(\mathbb{F}_{2} \oplus \mathbb{F}_{2}\right)^{\otimes|L|} \otimes \mathbb{F}_{2}\left[\eta, \eta^{-1}\right],
$$

and these spectral sequences are invariants of $L$. (Formulas (1.27) and (1.28) are proved in Theorem 7.16, though with slightly different variable names.)

Additional context. Other versions of equivariant Lagrangian intersection Floer homology substantially predate Seidel-Smith's work. In particular, Khovanov-Seidel [KS02, Section 5c] consider Floer homology in the presence of an involution, under hypotheses which allow one to achieve equivariant transversality. Equivariant versions of the Fukaya category of a symplectic manifold with an involution were studied by Seidel [Sei08, Chapter 14], again under restrictions on the Lagrangians which allow equivariant transversality. Equivariant Fukaya categories with respect to finite group actions were further developed by ChoHong [CH13] with coefficients in fields of characteristic 0, by averaging over multi-valued perturbations to avoid equivariant transversality issues. In a slightly different direction, the case of free actions has been exploited by Seidel [Sei15b, Section 8b] and Wu [Wu15].

Organization. This paper is organized as follows. In Section 2 we recall a little background about classical equivariant cohomology. In Section 3 we give a definition of the equivariant Floer complex which does not require equivariant transversality. When equivariant transversality can be achieved, we identify the new construction with the more obvious definition of equivariant Floer homology in Section 4, and prove Theorem 1.1. In Section 5 we turn to the first Heegaard Floer applications, to the spectral sequences (1.6) and (1.8), 
and prove Corollaries 1.12 and 1.13 and Theorems 1.14, 1.16, and 1.20, Corollaries 1.15 and 1.19, and Proposition 1.22. We conclude Section 3 by proving that equivariant Floer homology gives an invariant of $2^{m}$-fold covers. In Section 6 we construct the spectral sequence (1.23) and the concordance invariants $q_{\tau}(K)$ and $d_{\tau}(K, n)$ and study their basic properties. In Section 7 we turn to the Seidel-Smith spectral sequence, constructing the spectral sequences (1.27) and (1.28) proving Theorem 1.26, as well as Corollaries 1.4 and 1.5 .

Since some of the notions, like homotopy coherence, may be unfamiliar to some readers, we have added a number of explicit, elementary examples. To avoid interrupting the narrative flow of the paper, we have moved these examples to Appendix A, which the reader familiar with homotopy coherence is discouraged from reading. Other useful introductions to homotopy coherence include the books by Kamps-Porter [KP97, Chapter 5] and Lurie [Lur09].

Throughout this paper, all chain complexes are over the field $\mathbb{F}_{2}$ with two elements. Involutions will usually be denoted $\tau$.

Acknowledgments. We thank the contributors to nLab, which has been invaluable in sorting through the (to us unfamiliar) higher category theory literature. We also thank Mohammed Abouzaid, Aliakbar Daemi, Ailsa Keating, Tyler Lawson, Tye Lidman, Ciprian Manolescu, Davesh Maulik, Steven Sivek, Ivan Smith, Stephan Wehrli, and Jingyu Zhao for helpful conversations. Finally, we thank the referees for many helpful suggestions and corrections.

\section{BACKGROUND ON EQUIVARIANT COHOMOLOGY}

We briefly recall some homological algebra behind equivariant cohomology. Recall that our base ring is always the field $\mathbb{F}_{2}$ with two elements. Let $C_{*}$ be a chain complex (over $\mathbb{F}_{2}$ ) with a $\mathbb{Z} / 2$-action, or equivalently a chain complex over $\mathbb{F}_{2}[\mathbb{Z} / 2]$. Further assume that $C_{*}$ is bounded below, and is finite dimensional in each homological grading. The equivariant cohomology of $C_{*}$ is

$$
H_{\mathbb{Z} / 2}^{*}\left(C_{*}\right):=\operatorname{Ext}_{\mathbb{F}_{2}[\mathbb{Z} / 2]}\left(C_{*}, \mathbb{F}_{2}\right),
$$

where $\mathbb{Z} / 2$ acts trivially on $\mathbb{F}_{2}$. So, to compute $H_{\mathbb{Z} / 2}^{*}\left(C_{*}\right)$, one starts by taking, say, a projective resolution $\widetilde{C}_{*}$ of $C_{*}$ over $\mathbb{F}_{2}[\mathbb{Z} / 2]$ and then computing the homology of the complex

$$
\operatorname{RHom}_{\mathbb{F}_{2}[\mathbb{Z} / 2]}\left(C_{*}, \mathbb{F}_{2}\right):=\operatorname{Hom}_{\mathbb{F}_{2}[\mathbb{Z} / 2]}\left(\widetilde{C}_{*}, \mathbb{F}_{2}\right) .
$$

Writing $\mathbb{Z} / 2=\{1, \tau\}$, a particularly efficient projective resolution $\widetilde{C}_{*}$ is the total complex of the bicomplex

$$
\widetilde{C}_{*}:=\left(0 \longleftarrow C_{*} \otimes \mathbb{F}_{2}[\mathbb{Z} / 2] \stackrel{1+\tau}{\longleftarrow} C_{*} \otimes \mathbb{F}_{2}[\mathbb{Z} / 2] \stackrel{1+\tau}{\longleftarrow} \cdots\right) .
$$

for which the complex $\mathrm{RHom}_{\mathbb{F}_{2}[\mathbb{Z} / 2]}\left(C_{*}, \mathbb{F}_{2}\right)$ takes the form

$$
0 \longrightarrow C^{*} \stackrel{1+\tau}{\longrightarrow} C^{*} \stackrel{1+\tau}{\longrightarrow} C^{*} \stackrel{1+\tau}{\longrightarrow} \cdots .
$$

(Here by an abuse of notation $\tau$ also denotes the automorphism of $C^{*}$ induced by the $\mathbb{Z} / 2$ action.)

In the special case that $C_{*}$ is the singular chain complex of a space $X$ with a $\mathbb{Z} / 2$-action, the complex $C_{*}(X \times E \mathbb{Z} / 2)$ is a projective resolution of $C_{*}(X)$, so the equivariant cohomology as defined above agrees with the Borel equivariant cohomology of $X$. 
Given a module $M$ over $\mathbb{F}_{2}$, the $(\bmod 2)$ group cohomology of $\mathbb{Z} / 2$ with coefficients in $M$ is

$$
H^{*}(\mathbb{Z} / 2, M):=\operatorname{Ext}_{\mathbb{F}_{2}[\mathbb{Z} / 2]}\left(\mathbb{F}_{2}, M\right) .
$$

The special case $M=\mathbb{F}_{2}$ is simply called the group cohomology of $\mathbb{Z} / 2$ and written $H^{*}(\mathbb{Z} / 2):=H^{*}\left(\mathbb{Z} / 2, \mathbb{F}_{2}\right)=H_{\mathbb{Z} / 2}^{*}\left(\mathbb{F}_{2}\right)$. Composition gives a product on $H^{*}(\mathbb{Z} / 2)$ and an action of $H^{*}(\mathbb{Z} / 2)$ on $H_{\mathbb{Z} / 2}^{*}\left(C_{*}\right)$ for any complex $C_{*}$. An easy computation shows that $H^{*}(\mathbb{Z} / 2) \cong \mathbb{F}_{2}[\theta]$ where $\theta$ lies in degree 1 ; the action of $\theta$ on the complex $(2.2)$ is shifting it one unit to the right.

The horizontal filtration (or equivalently, the $\theta$-action) on the bicomplex (2.2) induces a spectral sequence with $E_{1}$-page given by

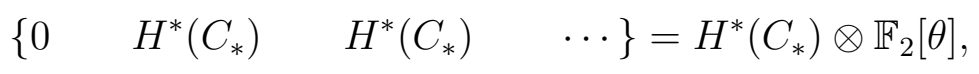

$E_{2}$-page given by $H^{*}\left(\mathbb{Z} / 2, H^{*}\left(C_{*}\right)\right)$, and $E_{\infty}$-page given by $H_{\mathbb{Z} / 2}^{*}\left(C_{*}\right)$.

One can recover the dual of the original chain complex from the equivariant version by simply setting $\theta=0$, namely, $C^{*} \cong \mathrm{RHom}_{\mathbb{F}_{2}[\mathbb{Z} / 2]}\left(C_{*}, \mathbb{F}_{2}\right) /(\theta=0)$. To be more precise, $C^{*}$ fits into a short exact sequence with the RHom complex from (2.2),

$$
0 \rightarrow \operatorname{RHom}_{\mathbb{F}_{2}[\mathbb{Z} / 2]}\left(C_{*}, \mathbb{F}_{2}\right) \stackrel{\cdot \theta}{\longrightarrow} \operatorname{RHom}_{\mathbb{F}_{2}[\mathbb{Z} / 2]}\left(C_{*}, \mathbb{F}_{2}\right) \stackrel{\pi}{\longrightarrow} C^{*} \rightarrow 0
$$

where $\pi$ is the projection to the leftmost $C^{*}$ in (2.2).

If $C_{*}^{\prime}$ is a chain complex over $\mathbb{F}_{2}[\mathbb{Z} / 2]$, and if $C_{*} \rightarrow C_{*}^{\prime}$ is a quasi-isomorphism over $\mathbb{F}_{2}[\mathbb{Z} / 2]$, we get an induced map of bicomplexes (2.2) so that the map of associated graded complexes is a quasi-isomorphism. Thus, we get induced isomorphisms between the entire spectral sequences $H^{*}\left(C_{*}^{\prime}\right) \otimes \mathbb{F}_{2}[\theta] \Rightarrow H_{\mathbb{Z} / 2}^{*}\left(C_{*}^{\prime}\right)$ and $H^{*}\left(C_{*}\right) \otimes \mathbb{F}_{2}[\theta] \Rightarrow H_{\mathbb{Z} / 2}^{*}\left(C_{*}\right)$. Furthermore, the induced map RHom $\mathbb{F}_{\mathbb{T}_{2}[\mathbb{Z} / 2]}\left(C_{*}^{\prime}, \mathbb{F}_{2}\right) \rightarrow \mathrm{RHom}_{\mathbb{F}_{2}[\mathbb{Z} / 2]}\left(C_{*}, \mathbb{F}_{2}\right)$ gives an isomorphism $H_{\mathbb{Z} / 2}^{*}\left(C_{*}^{\prime}\right) \stackrel{\cong}{\longrightarrow}$ $H_{\mathbb{Z} / 2}^{*}\left(C_{*}\right)$ on homology, as modules over $\mathbb{F}_{2}[\theta]$. These quasi-isomorphisms further produce quasi-isomorphisms between short exact sequences

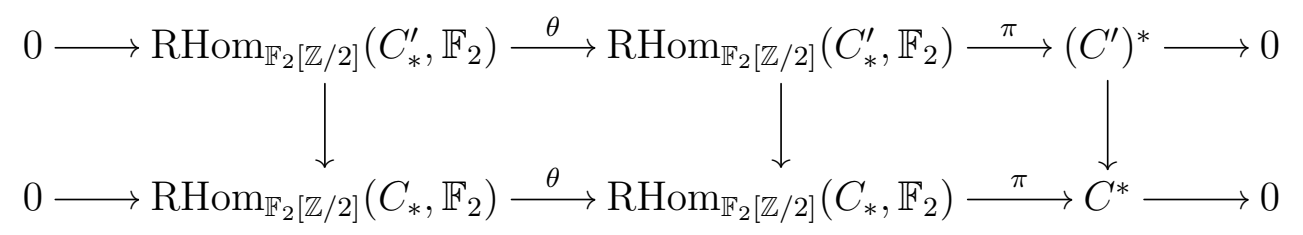

where the rightmost arrow is the dual of the original quasi-isomorphism between $C_{*}$ and $C_{*}^{\prime}$.

Similar constructions apply when $K$ is any finite group: given a chain complex $C_{*}$ with an action of $K$, we define

$$
\begin{aligned}
H_{K}^{*}\left(C_{*}\right) & :=\operatorname{Ext}_{\mathbb{F}_{2}[K]}\left(C_{*}, \mathbb{F}_{2}\right) \\
H^{*}(K, M) & :=\operatorname{Ext}_{\mathbb{F}_{2}[K]}\left(\mathbb{F}_{2}, M\right) \\
H^{*}(K) & :=H^{*}\left(K, \mathbb{F}_{2}\right)=H_{K}^{*}\left(\mathbb{F}_{2}\right) ;
\end{aligned}
$$

and $H_{K}^{*}\left(C_{*}\right)$ comes with an action of the algebra $H^{*}(K)$, by composition. There is no longer as nice a resolution as Formula (2.1), but choosing any particular free resolution $R_{*}$ of $\mathbb{F}_{2}$ over $\mathbb{F}_{2}[K]$ (for instance, the bar resolution) we get a resolution $C_{*} \otimes_{\mathbb{F}_{2}} R_{*}$ of $C_{*}$, and the grading on $R_{*}$ induces a filtration on this resolution. The $E_{1}$-page now depends on $R_{*}$, but the $E_{2}$-page is $H^{*}\left(K, H^{*}\left(C_{*}\right)\right)$. Also, there is no longer an easy way to recover the original chain complex from the equivariant version, but there is a spectral sequence $\operatorname{Tor}_{H^{*}(K)}\left(\mathbb{F}_{2}, H_{K}^{*}\left(C_{*}\right)\right) \Rightarrow H^{*}\left(C_{*}\right)$. 
Given a chain complex $C_{*}^{\prime}$ quasi-isomorphic to $C_{*}$ over $\mathbb{F}_{2}[K]$, the invariants $H_{K}^{*}\left(C_{*}\right)$ and $H_{K}^{*}\left(C_{*}^{\prime}\right)$ are isomorphic $H^{*}(K)$-modules, and each page (from the second on) of the spectral sequences $H^{*}\left(K, H^{*}\left(C_{*}\right)\right) \Rightarrow H_{K}^{*}\left(C_{*}\right)$ and $H^{*}\left(K, H^{*}\left(C_{*}^{\prime}\right)\right) \Rightarrow H_{K}^{*}\left(C_{*}^{\prime}\right)$ is isomorphic.

The upshot is that to prove equivariant cohomology, with its module structure, and the spectral sequence converging to it, is an invariant it suffices to prove that the original complex over $\mathbb{F}_{2}[K]$ is well-defined up to quasi-isomorphism over $\mathbb{F}_{2}[K]$.

\section{The Equivariant FloER COMPlEX VIA NON-EQUIVARIANT COMPLEX STRUCTURES}

Fix a symplectic manifold $(M, \omega)$, Lagrangians $L_{0}, L_{1} \subset M$, and a symplectic involution $\tau: M \rightarrow M$ preserving the $L_{i}$. In this section we discuss how to define the (Borel) equivariant Floer cohomology of $\left(L_{0}, L_{1}\right)$ without the need for equivariant transversality. Other constructions with similar invariance properties are given in [SS10, Section 2(a)] (written in the context of Morse theory) and [Sei15a, Section 3(b)] (in the context of fixed-point Floer homology).

Roughly, the idea is that the space of almost complex structures is contractible and admits an action by $\mathbb{Z} / 2$, and we will construct Floer cohomology parameterized by points in this space. There are many ways to formulate precisely what one means by Floer cohomology with this parameter space. In [SS10, Sei15a], the authors choose a Morse function on their parameter space and couple the $\bar{\partial}$ equation to the Morse flow equation on the space. Here, we take an approach more along the lines of simplicial sets, building a homotopy coherent functor from a category $\mathscr{E} \mathbb{Z} / 2$ (see Section 3.3) to the category of chain complexes, using an intermediary category of almost complex structures. One feature of this construction is that it generalizes easily from $\mathbb{Z} / 2$ to other finite groups.

3.1. Hypotheses and statement of result. While we do not require equivariant transversality, we do make assumptions which allow us to construct a $\mathbb{Z}$-graded Floer complex with coefficients in $\mathbb{F}_{2}$ (rather than a Novikov ring). Because we want Heegaard Floer invariants to fall under this rubric, we give fairly weak but intricate assumptions.

Convention 3.1. For the rest of Section 3, fix a collection $\eta$ of homotopy classes of paths from $L_{0}$ to $L_{1}$; by the Floer complex we mean the summand of the Floer complex spanned by intersection points which, viewed as constant paths from $L_{0}$ to $L_{1}$, lie in one of the homotopy classes in $\eta$; in Heegaard Floer terminology, this corresponds to fixing a collection of $\operatorname{spin}^{c}$ structures.

The hypotheses we require are:

Hypothesis 3.2. (J-1) For any loop of paths

$$
v:\left([0,1] \times S^{1},\{0\} \times S^{1},\{1\} \times S^{1}\right) \rightarrow\left(M, L_{0}, L_{1}\right)
$$

such that the homotopy class of the path $\left[\left.v\right|_{[0,1] \times\{p t\}}\right]$ is in $\eta$, the area of $v$ (with respect to $\omega$ ) and the Maslov index of $v$ both vanish. (Compare [WW12, Theorem 1.0.1].)

(Note that this condition holds for the hat version of the Heegaard Floer complex for admissible Heegaard diagrams; compare [OSz04b, Lemma 4.12].)

(J-2) The manifold $M$ is either compact or else is convex at infinity [EG91, Section 1.7.1]. Further, the Lagrangians $L_{0}$ and $L_{1}$ are either compact or else $M$ has finite type and $L_{0}$ and $L_{1}$ are conical at infinity and disjoint outside a compact set (see, e.g., [KS02, Section 5] or [Hen16, Section 2.1] and the references there). 
(J-3) There is an action of a finite group $H$ on $M$ by symplectomorphisms which preserve $L_{0}, L_{1}$, and the collection of homotopy classes of paths $\eta$ set-wise.

Under these hypotheses, we will define a model $\widetilde{C F}\left(L_{0}, L_{1}\right)$ for the Lagrangian Floer complex, which we will call the freed Floer complex (Definition 3.15 for $H=\mathbb{Z} / 2$ and Section 3.7 for arbitrary finite groups). The freed Floer complex is a module over $\mathbb{F}_{2}[H]$; regarded as simply a complex over $\mathbb{F}_{2}$ (by restriction of scalars), $\widetilde{C F}\left(L_{0}, L_{1}\right)$ is quasi-isomorphic to the usual Lagrangian Floer complex $C F\left(L_{0}, L_{1}\right)$. The construction depends on choices of (many) almost complex structures, but the resulting quasi-isomorphism type, over $\mathbb{F}_{2}[H]$, is independent of these choices (Propositions 3.23 and 3.28). Further, the equivariant quasiisomorphism type of $\widetilde{C F}\left(L_{0}, L_{1}\right)$ is invariant under Hamiltonian isotopies through equivariant Lagrangians (Proposition 3.24 and 3.28). Under additional hypotheses, $\widetilde{C F}\left(L_{0}, L_{1}\right)$ is even invariant under non-equivariant Hamiltonian isotopies (Propositions 3.25 and 3.29). Conveniently, $\widetilde{C F}\left(L_{0}, L_{1}\right)$ is a free module over $\mathbb{F}_{2}[H]$, so the equivariant Floer cochain complex $C F_{\mathbb{Z} / 2}\left(L_{0}, L_{1}\right)$ is simply $\operatorname{Hom}_{\mathbb{F}_{2}[H]}\left(\widetilde{C F}\left(L_{0}, L_{1}\right), \mathbb{F}_{2}\right)$, and the equivariant Floer cohomology $H F_{\mathbb{Z} / 2}\left(L_{0}, L_{1}\right)$ is $\operatorname{Ext}_{\mathbb{F}_{2}[H]}\left(\widetilde{C F}\left(L_{0}, L_{1}\right), \mathbb{F}_{2}\right)$. It follows from homological algebra that the isomorphism type of $H F_{\mathbb{Z} / 2}\left(L_{0}, L_{1}\right)$ and, in the case $H=\mathbb{Z} / 2$, the pages of the spectral sequence $H F^{*}\left(L_{0}, L_{1}\right) \otimes \mathbb{F}_{2}[\theta] \Rightarrow H F_{\mathbb{Z} / 2}\left(L_{0}, L_{1}\right)$, are invariants of $\left(L_{0}, L_{1}\right)$ up to equivariant Hamiltonian isotopy.

3.2. The extended space of eventually cylindrical almost complex structures. By a cylindrical complex structure on $(M, \omega)$ we mean a smooth, one-parameter family $J=J(t)$, $t \in[0,1]$, of smooth almost complex structures on $M$ so that each $J(t)$ is compatible with $\omega$. Let $\mathcal{J}_{\text {cyl }}$ denote the space of cylindrical almost complex structures with the $C^{\infty}$ topology. By an eventually cylindrical almost complex structure on $(M, \omega)$ we mean a smooth map $\widetilde{J}: \mathbb{R} \rightarrow \mathcal{J}_{\text {cyl }}$ which is constant outside some compact set, modulo the equivalence relation that for any $s_{0} \in \mathbb{R}, \widetilde{J}(\cdot) \sim \widetilde{J}\left(\cdot+s_{0}\right.$ ) (i.e., modulo translation in the source). Let $\mathcal{J}$ denote the set of eventually cylindrical almost complex structures.

Define a topology on $\mathcal{J}$ as follows. Given a compact interval $I \subset \mathbb{R}$ and an open set $V$ in the space of smooth maps $\mathbb{R} \times[0,1] \times T M \rightarrow T M$ (with the $C^{\infty}$ topology), let $U(I, V)$ be the set of $\widetilde{J} \in \mathcal{J}$ so that there is a representative for $\widetilde{J}$ which is constant on $\mathbb{R} \backslash I$ and which, when viewed as a map $\mathbb{R} \times[0,1] \times T M \rightarrow T M$, lies in $V$. Define the topology on $\mathcal{J}$ to be generated by the sub-basis consisting of all such sets $U(I, V)$. In particular, a sequence $\left\{\widetilde{J}_{i}\right\}$ converges if, for some representatives of the equivalence classes of the $\widetilde{J}_{i}$, there is a compact set $I \subset \mathbb{R}$ so that all of the $\widetilde{J}_{i}$ are constant on each component of $\mathbb{R} \backslash I$ and the $\left.\widetilde{J}_{i}\right|_{I}$ converge in the $C^{\infty}$ topology.

An eventually cylindrical almost complex structure $\widetilde{J}$ specifies a cylindrical almost complex structure $J_{-\infty}$ (respectively $\left.J_{+\infty}\right)$ near $-\infty$ (respectively $\left.+\infty\right)$. Let $\mathcal{J}\left(J_{-\infty}, J_{+\infty}\right)$ be the subspace of $\mathcal{J}$ specifying $J_{ \pm \infty}$ near $\pm \infty$, respectively. (The $\mathcal{J}\left(J_{-\infty}, J_{+\infty}\right)$ 's are the path components of $\mathcal{J}$.) If $\widetilde{J} \in \mathcal{J}\left(J_{-\infty}, J_{+\infty}\right)$ then we may write $\left.\widetilde{J}\right|_{-\infty}=J_{-\infty}$ and $\left.\widetilde{J}\right|_{+\infty}=J_{+\infty}$. Note that $\mathcal{J}_{\text {cyl }} \subset \mathcal{J}$ as the constant maps $\mathbb{R} \rightarrow \mathcal{J}_{\text {cyl }}$.

It will be convenient to add some extra points to $\mathcal{J}$, corresponding to multi-story almost complex structures. Specifically, let $\overline{\overline{\mathcal{J}}}\left(J, J^{\prime}\right)$ denote the set of finite, non-empty sequences

$$
\left(\widetilde{J}^{1}, \widetilde{J}^{2}, \ldots, \widetilde{J}^{n}\right) \in \mathcal{J}\left(J=J_{0}, J_{1}\right) \times \mathcal{J}\left(J_{1}, J_{2}\right) \times \cdots \times \mathcal{J}\left(J_{n-1}, J_{n}=J^{\prime}\right)
$$


of eventually cylindrical complex structures so that $\left.\widetilde{J}^{i}\right|_{+\infty}=\left.\widetilde{J}^{i+1}\right|_{-\infty}$, starting at $J$ and ending at $J^{\prime}$. Define an equivalence relation $\sim$ on $\overline{\overline{\mathcal{J}}}$ be declaring that

$$
\left(\widetilde{J}^{1}, \ldots, \widetilde{J}^{i-1}, \widetilde{J}^{i}, \widetilde{J}^{i+1}, \ldots \widetilde{J}^{n}\right) \sim\left(\widetilde{J}^{1}, \ldots, \widetilde{J}^{i-1}, \widetilde{J}^{i+1}, \ldots \widetilde{J}^{n}\right)
$$

if $\widetilde{J}^{i} \in \mathcal{J}_{\text {cyl }}$. Let $\overline{\mathcal{J}}$ be the quotient of $\overline{\overline{\mathcal{J}}}$ by the equivalence relation $\sim$.

There is a composition operation $\circ: \overline{\mathcal{J}}\left(J_{2}, J_{3}\right) \times \overline{\mathcal{J}}\left(J_{1}, J_{2}\right) \rightarrow \overline{\mathcal{J}}\left(J_{1}, J_{3}\right)$ defined by $\widetilde{J}^{2} \circ \widetilde{J}^{1}=$ $\left(\widetilde{J}^{1}, \widetilde{J}^{2}\right)$.

Next, we define a topology on $\overline{\mathcal{J}}$. Fix a (one-story) cylindrical-at-infinity almost complex structure $\widetilde{J}$ (or rather, a representative of the equivalence class $\widetilde{J}$ ) and real numbers $a_{1}<$ $b_{1}<a_{2}<b_{2}<\cdots<a_{k}<b_{k}$ so that $\widetilde{J}$ is locally constant in the $\mathbb{R}$ coordinate except on the intervals $\left[a_{i}, b_{i}\right], 1 \leq i \leq k$. Say that a cylindrical-at-infinity almost complex structure $\widetilde{J}^{\prime}$ is obtained from $\left(\widetilde{J},\left\{a_{i}, b_{i}\right\}\right)$ by an elementary expansion if there is some $1 \leq j<k$ and an $r \in \mathbb{R}$ so that for $t \in \mathbb{R}$,

$$
\widetilde{J}^{\prime}(t)= \begin{cases}\widetilde{J}(t) & \text { if } t \leq b_{i} \\ \widetilde{J}\left(b_{i}\right)=\widetilde{J}\left(a_{i+1}\right) & \text { if } b_{i} \leq t \leq a_{i+1}+r \\ \widetilde{J}(t-r) & \text { if } t \geq a_{i+1}\end{cases}
$$

We also say that the two-story almost complex structure $\widetilde{J}^{\prime}$ whose first story $\widetilde{J}^{1}$ is given by

$$
\widetilde{J}^{1}(t)= \begin{cases}\widetilde{J}(t) & \text { if } t \leq b_{i} \\ \widetilde{J}\left(b_{i}\right) & \text { if } t \geq b_{i}\end{cases}
$$

and whose second story $\widetilde{J}^{2}$ is given by

$$
\widetilde{J}^{2}(t)= \begin{cases}\widetilde{J}\left(b_{i}\right) & \text { if } t \leq a_{i+1} \\ \widetilde{J}(t) & \text { if } t \geq a_{i+1}\end{cases}
$$

is obtained from $\widetilde{J}$ by an elementary expansion. Note that we have a distinguished collection of points $a_{1}^{\prime}, b_{1}^{\prime}, \ldots, a_{k}^{\prime}, b_{k}^{\prime}$ so that $\widetilde{J}^{\prime}$ is constant on $\left[b_{i}^{\prime}, a_{i+1}^{\prime}\right]$. (Here, we abuse notation slightly in the case that $\widetilde{J}^{\prime}$ has two stories.) More generally, consider a pair $\left(\widetilde{J},\left\{a_{i}, b_{i}\right\}\right)$, where $\widetilde{J}$ is a multi-story almost complex structure and the $a_{1}<b_{1}<\cdots<a_{k}<b_{k}$ are a sequence of points in the source of $\widetilde{J}$ (a sequence of copies of $\mathbb{R}$ ). We say that a multi-story almost complex structure $\left(\widetilde{J}^{\prime},\left\{a_{i}^{\prime}, b_{i}^{\prime}\right\}\right)$ is obtained from $\left(\widetilde{J},\left\{a_{i}, b_{i}\right\}\right)$ by an elementary expansion if $\widetilde{J}^{\prime}$ is obtained by replacing some story in $\widetilde{J}$ by an elementary expansion of that story and $\left\{a_{i}^{\prime}, b_{i}^{\prime}\right\}$ are the new distinguished points. Finally, we say that $\widetilde{J}^{\prime}$ is obtained from $\left(\widetilde{J},\left\{a_{i}, b_{i}\right\}\right)$ by an expansion if $\widetilde{J}^{\prime}$ is obtained by some sequence of elementary expansions. Informally, $\widetilde{J}^{\prime}$ is obtained from $\widetilde{J}$ by an expansion if $\widetilde{J}^{\prime}$ comes from making some of the distinguished intervals on which $\widetilde{J}$ is constant longer, including allowing those intervals to become infinitely long.

Now, fix an open neighborhood $V$ of $\widetilde{J}$ in $\mathcal{J}$ and $2 n$ points $\left\{a_{i}, b_{i}\right\}$ in $\mathbb{R}$ (for some $n$ ) so that each $\widetilde{J}^{\prime} \in V$ is constant on each component of $\mathbb{R} \backslash \bigcup_{i=1}^{n}\left[a_{i}, b_{i}\right]$, and consider the set $U\left(\widetilde{J}, V,\left\{a_{i}, b_{i}\right\}\right)$ of multi-story almost complex structures which are obtained from $\left(\widetilde{J^{\prime}},\left\{a_{i}, b_{i}\right\}\right)$ for some $\widetilde{J}^{\prime} \in V$ by an expansion. The sets $U\left(\widetilde{J}, V,\left\{a_{i}, b_{i}\right\}\right)$ form a sub-basis for the topology on $\overline{\mathcal{J}}$. 
Note, in particular, that if $\left\{a_{i}, b_{i}\right\}$ has $2 n$ elements (i.e., $n$ intervals) then any complex structure in $U\left(\widetilde{J}, V,\left\{a_{i}, b_{i}\right\}\right)$ has at most $n$ stories. So, the space $\mathcal{J}$ is an open subset of $\overline{\mathcal{J}}$.

It is perhaps clearer to spell out what it means for a sequence of almost complex structures in $\overline{\mathcal{J}}$ to converge. Given a sequence $\widetilde{J}^{i}$ in $\mathcal{J}$, we say that $\widetilde{J}^{i}$ converges to a point $\left(\widetilde{J}^{1}, \ldots, \widetilde{J}^{n}\right) \in$ $\overline{\mathcal{J}}$ if for some representatives of the $\widetilde{J}^{i}$ there is a sequence of $n$-tuples of open intervals $\left(I_{1}^{i}, \ldots, I_{n}^{i}\right)$ in $\mathbb{R}, i=1, \ldots, \infty$, so that:

- For each sufficiently large $i$, the $I_{k}^{i}$ are disjoint.

- For each $k$, the length of $I_{k}^{i}$ is bounded independently of $i$.

- The distance between $I_{k}^{i}$ and $I_{k+1}^{i}$ goes to infinity as $i \rightarrow \infty$.

- Each $\widetilde{J}^{i}$ is constant on each component of $\mathbb{R} \backslash\left(I_{1}^{i} \cup \cdots \cup I_{n}^{i}\right)$.

- Let $\widetilde{J}^{i, k}$ be the result of restricting $\widetilde{J}^{i}$ to $I_{k}$ and extending by the constant map, to get an eventually cylindrical almost complex structure on all of $\mathbb{R}$. Then we require that for each $k$, the $\widetilde{J}^{i, k}$ converge to $\widetilde{J}^{k}$.

Like $\mathcal{J}, \overline{\mathcal{J}}$ is a disjoint union of path components $\overline{\mathcal{J}}\left(J_{-\infty}, J_{+\infty}\right)$.

As the composition notation suggests, the space $\overline{\mathcal{J}}$ is a topological category. The objects of $\overline{\mathcal{J}}$ are the cylindrical almost complex structures, $\mathcal{J}_{\text {cyl }}$, $\operatorname{Hom}\left(J_{1}, J_{2}\right)=\overline{\mathcal{J}}\left(J_{1}, J_{2}\right)$, which is a topological space, and composition is $\circ$, which is continuous. The identity map of any cylindrical almost complex structure $J$ is the constant path at $J$; because of the equivalence relation we imposed, this is a strict identity.

If there is a symplectic action of a group $G$ on $M$ then there is an induced action of $G$ on $\overline{\mathcal{J}}$ by functors; $g_{*}(\widetilde{J})=d g \circ \widetilde{J} \circ(d g)^{-1}$.

It is well known that the space of almost complex structures on $M$ compatible with $\omega$ is contractible. It follows immediately that $\mathcal{J}_{\text {cyl }}$ is contractible. Further, it is not hard to see that $\mathcal{J}\left(J_{-\infty}, J_{+\infty}\right)$ and $\overline{\mathcal{J}}\left(J_{-\infty}, J_{+\infty}\right)$ are at least weakly contractible (i.e., have trivial homotopy groups). (This is used in the proof Lemma 3.13.)

3.3. Homotopy coherent diagrams of almost complex structures. To build our equivariant complex we will need to fix a number of choices. Let $\mathscr{E} \mathbb{Z} / 2$ denote the category with two objects, $a$ and $b$, with $\operatorname{Hom}(x, y)$ a single element for any $x, y \in\{a, b\}$. (The category $\mathscr{E} \mathbb{Z} / 2$ is a groupoid.) Graphically:

$$
\mathscr{E} \mathbb{Z} / 2=\mathbb{I} C a \stackrel{\longleftarrow_{\alpha}^{\beta}}{\zeta} \supset \mathbb{I}
$$

There is an obvious $\mathbb{Z} / 2$-action on $\mathscr{E} \mathbb{Z} / 2$, exchanging $a$ and $b$.

The choices we will need in order to define the equivariant complex can be summarized as a generic, $\mathbb{Z} / 2$-equivariant homotopy coherent diagram $\mathscr{E} \mathbb{Z} / 2 \rightarrow \overline{\mathcal{J}}$. Given a category $\mathscr{C}$ and a homotopy coherent diagram $\mathscr{C} \rightarrow \overline{\mathcal{J}}$, we can apply Floer theory to obtain a homotopy coherent diagram from $\mathscr{C}$ to simplicial abelian groups (i.e., chain complexes). In particular, the homotopy colimit of this diagram is a chain complex. In the case of the $\mathbb{Z} / 2$-equivariant diagram $\mathscr{E} \mathbb{Z} / 2 \rightarrow \overline{\mathcal{J}}$, we get a chain complex over $\mathbb{F}_{2}[\mathbb{Z} / 2]$ which, because the action of $\mathbb{Z} / 2$ on $\mathscr{E} \mathbb{Z} / 2$ is free, is a free module. Taking Hom to the trivial $\mathbb{F}_{2}[\mathbb{Z} / 2]$-module $\mathbb{F}_{2}$ and taking homology yields the desired equivariant cohomology. The chain complex whose homology is the equivariant Floer cohomology is given explicitly in Observation 3.16.

We explain this in a little more detail next. In this section, we focus on diagrams $\mathscr{C} \rightarrow \overline{\mathcal{J}}$, and restrict to the special case of equivariant diagrams $\mathscr{E} \mathbb{Z} / 2 \rightarrow \overline{\mathcal{J}}$ in Section 3.4. Roughly, 
a homotopy coherent diagram is a homotopy commutative diagram in which the homotopies are part of the data, and commute up to appropriate higher homotopies (also part of the data). The first precise definition of this notion was given by Vogt:

Definition 3.3. [Vog73, Definition 2.3] Given a small category $\mathscr{C}$, a homotopy coherent $\mathscr{C}$-diagram in $\overline{\mathcal{J}}$ consists of:

- For each object $x$ of $\mathscr{C}$, an object $F(x)$ of $\overline{\mathcal{J}}$, i.e., a cylindrical almost complex structure.

- For each integer $n \geq 1$ and each sequence $x_{0} \stackrel{f_{1}}{\longrightarrow} \cdots \stackrel{f_{n}}{\longrightarrow} x_{n}$ of composable morphisms a continuous map $F\left(f_{n}, \ldots, f_{1}\right):[0,1]^{n-1} \rightarrow \overline{\mathcal{J}}\left(F\left(x_{0}\right), F\left(x_{n}\right)\right)$

such that

$$
\begin{gathered}
F\left(f_{n}, \ldots, f_{2}, \mathbb{I}\right)\left(t_{1}, \ldots, t_{n-1}\right)=F\left(f_{n}, \ldots, f_{2}\right)\left(t_{2}, \ldots, t_{n-1}\right) \\
F\left(\mathbb{I}, f_{n-1}, \ldots, f_{1}\right)\left(t_{1}, \ldots, t_{n-1}\right)=F\left(f_{n-1}, \ldots, f_{1}\right)\left(t_{1}, \ldots, t_{n-2}\right) \\
F\left(f_{n}, \ldots, f_{i+1}, \mathbb{I}, f_{i-1}, \ldots, f_{1}\right)\left(t_{1}, \ldots, t_{n-1}\right) \\
=F\left(f_{n}, \ldots, f_{i+1}, f_{i-1}, \ldots, f_{1}\right)\left(t_{1}, \ldots, t_{i-1} \cdot t_{i}, \ldots, t_{n-1}\right) \\
F\left(f_{n}, \ldots, f_{1}\right)\left(t_{1}, \ldots, t_{i-1}, 1, t_{i+1}, \ldots, t_{n-1}\right) \\
=F\left(f_{n}, \ldots, f_{i+1} \circ f_{i}, \ldots, f_{1}\right)\left(t_{1}, \ldots, t_{i-1}, t_{i+1}, \ldots, t_{n-1}\right) \\
F\left(f_{n}, \ldots, f_{1}\right)\left(t_{1}, \ldots, t_{i-1}, 0, t_{i+1}, \ldots, t_{n-1}\right) \\
=\left[F\left(f_{n}, \ldots, f_{i+1}\right)\left(t_{i+1}, \ldots, t_{n-1}\right)\right] \circ\left[F\left(f_{i}, \ldots, f_{1}\right)\left(t_{1}, \ldots, t_{i-1}\right)\right] .
\end{gathered}
$$

$\left(\right.$ Here, $\left(t_{1}, \ldots, t_{n-1}\right) \in[0,1]^{n-1}$.)

We will often drop the words "homotopy coherent," and simply refer to a homotopy coherent $\mathscr{C}$-diagram as a $\mathscr{C}$-diagram or as a diagram $F: \mathscr{C} \rightarrow \overline{\mathcal{J}}$.

Remark 3.4. A strictly commutative diagram is the special case that

$$
F\left(f_{n}, \ldots, f_{1}\right)\left(t_{1}, \ldots, t_{n-1}\right)=F\left(f_{n} \circ \cdots \circ f_{1}\right),
$$

for all $t_{1}, \ldots, t_{n-1}$. (This example is important in Section 4.)

See Example A.1 for an explicit example of the first few terms in a homotopy coherent $\mathscr{E} \mathbb{Z} / 2$-diagram in $\overline{\mathcal{J}}$.

Definition 3.5. Given a point $\widetilde{J}=\left(\widetilde{J}^{1}, \widetilde{J}^{2}, \ldots, \widetilde{J}^{n}\right)$ in $\overline{\mathcal{J}}$, where each $\widetilde{J}^{i} \in \mathcal{J}\left(J_{i-1}, J_{i}\right)$ is not $\mathbb{R}$-invariant, and points $x, y \in L_{0} \cap L_{1}$, by a $\widetilde{J}$-holomorphic disk from $x$ to $y$ we mean $a$ sequence

$$
\left(v^{0,1}, \ldots, v^{0, m_{0}}, u^{1}, v^{1,1}, \ldots, v^{1, m_{1}}, u^{2}, \ldots, u^{n}, v^{n, 1}, \ldots, v^{n, m_{n}}\right)
$$

where

- each $v^{i, j}$ is a $J_{i}$-holomorphic Whitney disk with boundary on $L_{0}$ and $L_{1}$ connecting some points $x^{i, j-1}$ and $x^{i, j}$ in $L_{0} \cap L_{1}$,

- each $u^{i}$ is a $\widetilde{J}_{i}$-holomorphic Whitney disk with boundary on $L_{0}$ and $L_{1}$ connecting some points $x^{i-1}$ and $x^{i}$ in $L_{0} \cap L_{1}$,

- for $i=1, \ldots, n, x^{i, 0}=x^{i}$,

- for $i=0, \ldots, n-1, x^{i, m_{i}}=x^{i+1}$,

- $x^{0,0}=x$, and $x^{n, m_{n}}=y$. 
(The numbers $m_{i}$ are allowed to be zero, i.e., the $v^{i, j}$ are not required to appear.)

Given a map $\widetilde{J}=\widetilde{J}\left(t_{1}, \ldots, t_{k}\right):[0,1]^{k} \rightarrow \overline{\mathcal{J}}$ and points $x, y \in L_{0} \cap L_{1}$ let $\mathcal{M}(x, y ; \widetilde{J})$ denote the moduli space of pairs $(\vec{t}, u)$ where $\vec{t} \in[0,1]^{k}$ and $u$ is a $\widetilde{J}(\vec{t})$-holomorphic disk from $x$ to $y$.

We say that a diagram $F: \mathscr{C} \rightarrow \overline{\mathcal{J}}$ is sufficiently generic if for any sequence of composable arrows $\left(f_{1}, \ldots, f_{n}\right)$ in $\mathscr{C}$ and any $x, y \in L_{0} \cap L_{1}$, the moduli space $\mathcal{M}\left(x, y ; F\left(f_{n}, \ldots, f_{1}\right)\right)$ is transversely cut out by the $\bar{\partial}$-equation.

Note that the space $\mathcal{M}(x, y ; \widetilde{J})$ decomposes as a union

$$
\mathcal{M}(x, y ; \widetilde{J})=\bigcup_{\phi \in \pi_{2}(x, y)} \mathcal{M}(\phi ; \widetilde{J})
$$

according to homotopy classes of Whitney disks. If $\widetilde{J}$ is a $k$-parameter family of almost complex structures then the expected dimension of $\mathcal{M}(\phi ; \widetilde{J})$ is $\mu(\phi)+k$, where $\mu$ is the Maslov index.

Lemma 3.6. Let $F: \mathscr{C} \rightarrow \overline{\mathcal{J}}$ be a diagram and suppose that for each object $x \in \mathrm{Ob}(\mathscr{C})$ the moduli spaces of disks with respect to the cylindrical complex structure $F(x)$ are transversely cut out. Then there is a sufficiently generic $F^{\prime}: \mathscr{C} \rightarrow \overline{\mathcal{J}}$ arbitrarily close to $F$. Further, if the restriction of $F\left(f_{m}, \ldots, f_{1}\right)$ is already sufficiently generic for all sequences $\left(f_{1}, \ldots, f_{m}\right)$ of length $m \leq n$ then one can choose $F^{\prime}$ to agree with $F$ for all such sequences.

Proof sketch. This follows from standard transversality arguments, which are explained nicely by McDuff-Salamon in a closely related setting [MS04, Chapter 3]. We outline the key steps.

The proof is by induction on $n$, the length of the sequence, as in the second half of the lemma's statement. Suppose that a sufficiently generic $F^{\prime}$ has been chosen already for all sequences of length $m<n$. Consider a sequence $\left(f_{1}, \ldots, f_{n}\right)$ of non-identity morphisms. Then $F^{\prime}\left(f_{n}, \ldots, f_{1}\right)$ should be a family of almost complex structures parameterized by $[0,1]^{n-1}$, close to $F\left(f_{n}, \ldots, f_{1}\right)$, and so that the restriction $\left.F^{\prime}\left(f_{n}, \ldots, f_{1}\right)\right|_{\partial\left([0,1]^{n-1}\right)}$ of $F^{\prime}$ to the boundary of the cube is determined by Definition 3.3.

Note that the condition of being sufficiently generic is local, in the following sense: given an open set $U \subset[0,1]^{n-1}$, and $F^{\prime}: U \rightarrow \overline{\mathcal{J}}$, it makes sense to ask whether $F^{\prime}$ is sufficiently generic on $U$, i.e., whether the moduli space $\mathcal{M}\left(x, y ;\left.F^{\prime}\right|_{U}\right)$ is transversely cut out.

Let $U$ be a small neighborhood of $\partial\left([0,1]^{n-1}\right)$ so that the boundary of $U$ consists of the boundary of $[0,1]^{n-1}$ and a smooth sphere $S^{n-2}$. Extend $F^{\prime}$ to $U$ continuously, and so that on $U \backslash \partial\left([0,1]^{n-1}\right)$ the map $F^{\prime}$ takes values $\mathcal{J}$, the space of 1 -story cylindrical-at-infinity almost complex structures. By shrinking $U$ we may arrange that $F^{\prime}$ is arbitrarily close to $F$ on $U$. If $U$ is small enough, then the $F^{\prime}$ is sufficiently generic over $U:\left.F^{\prime}\right|_{\partial\left([0,1]^{n-1}\right)}$ is sufficiently generic by induction, and it is not hard to show that the condition of being sufficiently generic is open.

Now, let $V$ be the inside of $S^{n-2}$, i.e., the component of $[0,1]^{n-1} \backslash S^{n-2}$ on which $F^{\prime}$ has not yet been defined. The next step is to use Sard's theorem to extend $F^{\prime}$ to $V$. Given intersection points $x, y \in L_{0} \cap L_{1}$, let $\mathcal{B}(x, y)$ be an appropriate weighted Sobolev space of maps

$$
(\mathbb{R} \times[0,1], \mathbb{R} \times\{0\}, \mathbb{R} \times\{1\}) \rightarrow\left(M, L_{0}, L_{1}\right)
$$

converging exponentially to $x$ at $-\infty$ and $y$ at $+\infty$. (See, for example, Seidel [Sei08, Section (8h)] for details on the functional analytic setup.) There is also a space $\operatorname{Map}(\bar{V}, \mathcal{J})$ of maps 
$\bar{V} \rightarrow \mathcal{J}$ extending $\left.F^{\prime}\right|_{S^{n-2}}$, which we topologize as a subspace of the space of $C^{\ell}$ maps $\bar{V} \times[0,1] \times T M \rightarrow T M$, for an appropriate $\ell$. Finally, there is a bundle $\mathcal{E} \rightarrow \mathcal{B}(x, y) \times$ $\operatorname{Map}(\bar{V}, \mathcal{J}) \times \bar{V}$ whose fiber over $(u, J, p)$ is a weighted Sobolev space of $(0,1)$-forms on $[0,1] \times \mathbb{R}$ (with respect to $J(p)$ ) valued in $u^{*} T M$. The universal $\bar{\partial}$-operator is a section

$$
\bar{\partial}: \mathcal{B}(x, y) \times \operatorname{Map}(\bar{V}, \mathcal{J}) \times \bar{V} \rightarrow \mathcal{E} .
$$

It follows from the argument given in McDuff-Salamon [MS04, Proof of Proposition 3.2.1] that the operator $\bar{\partial}$ is transverse to the 0 -section. Thus, the universal moduli space $\bar{\partial}^{-1}(0)$ is a $C^{k}$ Banach manifold (where $k$ depends on $\ell$ and the Maslov index difference between $x$ and $y$ ). Any regular value of the projection $\bar{\partial}^{-1}(0) \rightarrow \operatorname{Map}(\bar{V}, \mathcal{J})$ is a sufficiently generic almost complex structure. Smale's infinite dimensional version of Sard's theorem guarantees that the set of regular values is dense, so, in particular, we can find a regular value arbitrarily close to $\left.F\right|_{V}$.

Taking $\left.F^{\prime}\right|_{V}$ to be such a regular value gives a $C^{k}$ sufficiently generic almost complex structure as close as desired to $F$. While $C^{k}$ almost complex structures for finite $k$ are enough for the applications in this paper, a short further argument as in McDuff-Salamon [MS04, Proof of Theorem 3.1.6(II)] guarantees the existence of generic smooth complex structures, as well.

Notice that in order to define a homotopy coherent diagram, the key ingredients we needed about $\overline{\mathcal{J}}$ (or any other topological category) were the product and the interval $[0,1]$. We can give an analogous definition in the category of chain complexes, using $\otimes$ in place of the product and the chain complex

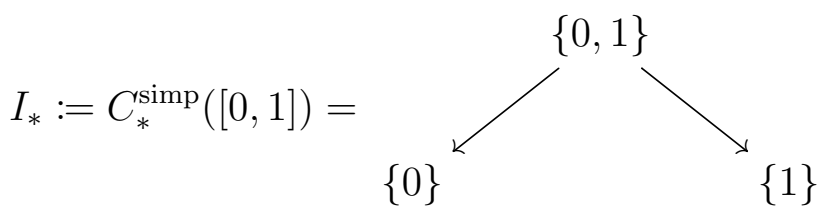

in place of $[0,1]$. The inclusions $\{0\} \hookrightarrow[0,1]$ and $\{1\} \hookrightarrow[0,1]$ induce chain maps $\iota_{0}, \iota_{1}$ : $\mathbb{F}_{2} \hookrightarrow I_{*}$ and the projection $[0,1] \rightarrow\{p t\}$ induces a chain map $\pi: I_{*} \rightarrow \mathbb{F}_{2}$. The multiplication map $[0,1]^{2} \rightarrow[0,1],(x, y) \mapsto x y$ induces the "multiplication" $m: I_{*} \otimes I_{*} \rightarrow I_{*}$ given by

$$
\begin{array}{ll}
m(\{0\} \otimes\{0\})=m(\{0\} \otimes\{1\})=m(\{1\} \otimes\{0\})=\{0\} & m(\{1\} \otimes\{1\})=\{1\} \\
m(\{0,1\} \otimes\{0\})=m(\{0\} \otimes\{0,1\})=0 & m(\{0,1\} \otimes\{1\})=m(\{1\} \otimes\{0,1\})=\{0,1\} \\
m(\{0,1\} \otimes\{0,1\})=0 . &
\end{array}
$$

(For a formal setting which generalizes both examples, see for instance [KP97, Chapter $5]$ ). We spell out the notion of homotopy coherent diagrams:

Definition 3.7. A homotopy coherent $\mathscr{C}$-diagram in chain complexes consists of:

- For each object $x$ of $\mathscr{C}$, a chain complex $G(x)$.

- For each $n \geq 1$ and each sequence $x_{0} \stackrel{f_{1}}{\longrightarrow} \cdots \stackrel{f_{n}}{\longrightarrow} x_{n}$ of composable morphisms a chain map $G\left(f_{n}, \ldots, f_{1}\right): I_{*}^{\otimes(n-1)} \otimes G\left(x_{0}\right) \rightarrow G\left(x_{n}\right)$ 
such that

$$
\begin{aligned}
& G\left(f_{n}, \ldots, f_{1}\right)\left(t_{1} \otimes \cdots \otimes t_{n-1}\right) \\
& = \begin{cases}G\left(f_{n}, \ldots, f_{2}\right)\left(\pi\left(t_{1}\right) \otimes t_{2} \otimes \cdots \otimes t_{n-1}\right) & f_{1}=\mathbb{I} \\
G\left(f_{n}, \ldots, f_{i+1}, f_{i-1}, \ldots, f_{1}\right)\left(t_{1} \otimes \cdots \otimes m\left(t_{i-1} \otimes t_{i}\right) \otimes \cdots \otimes t_{n-1}\right) & f_{i}=\mathbb{I}, 1<i<n \\
G\left(f_{n-1}, \ldots, f_{1}\right)\left(t_{1} \otimes \cdots \otimes t_{n-2} \otimes \pi\left(t_{n-1}\right)\right) & f_{n}=\mathbb{I} \\
G\left(f_{n}, \ldots, f_{i+1} \circ f_{i}, \ldots, f_{1}\right)\left(t_{1} \otimes \cdots \otimes t_{i-1} \otimes t_{i+1} \otimes \cdots \otimes t_{n-1}\right) & t_{i}=\{1\} \\
{\left[G\left(f_{n}, \ldots, f_{i+1}\right)\left(t_{i+1} \otimes \cdots \otimes t_{n-1}\right)\right] \circ\left[G\left(f_{i}, \ldots, f_{1}\right)\left(t_{1} \otimes \cdots \otimes t_{i-1}\right)\right]} & t_{i}=\{0\} .\end{cases} \\
& \text { (Here, } \left.\left(t_{1} \otimes \cdots \otimes t_{n-1}\right) \in I_{*}^{\otimes(n-1)} .\right)
\end{aligned}
$$

As in the case of diagrams in $\overline{\mathcal{J}}$, we will usually drop the words "homotopy coherent", and refer to homotopy coherent diagrams in chain complexes simply as diagrams $G: \mathscr{C} \rightarrow \mathrm{Kom}$.

Observe that a homotopy coherent $\mathscr{C}$-diagram of chain complexes is determined by the complexes $G(x)$ and the maps

$$
G_{f_{n}, \ldots, f_{1}}=G\left(f_{n}, \ldots, f_{1}\right)(\{0,1\} \otimes \cdots \otimes\{0,1\}): G(x)_{m} \rightarrow G(y)_{m+n-1}
$$

where $x$ is the source of $f_{1}, y$ is the target of $f_{n}$, and the subscripts on $G(x)$ and $G(y)$ indicate the gradings. These maps satisfy compatibility conditions

- $G_{\mathbb{I}_{x_{0}}}=\mathbb{I}_{G\left(x_{0}\right)}$

- $G_{f_{n}, \ldots, f_{1}}=0$ if $n>1$ and any $f_{i}=\mathbb{I}$

- $\partial \circ G_{f_{n}, \ldots, f_{1}}=G_{f_{n}, \ldots, f_{1}} \circ \partial+\sum_{i=1}^{n-1} G_{f_{n}, \ldots, f_{i+1} \circ f_{i}, \ldots, f_{1}}+\sum_{i=1}^{n-1} G_{f_{n}, \ldots, f_{i+1}} \circ G_{f_{i}, \ldots, f_{1}}$.

See Example A.2 for the first few terms in a homotopy coherent $\mathscr{E} \mathbb{Z} / 2$-diagram in Kom.

A homotopy coherent diagram $G$ is called strict if $G_{f_{n}, \ldots, f_{1}}=0$ whenever $n>1$.

Given a sufficiently generic $\mathscr{C}$-diagram $F$ in $\overline{\mathcal{J}}$, Floer theory produces a homotopy coherent diagram of chain complexes.

Construction 3.8. Fix a sufficiently generic $F: \mathscr{C} \rightarrow \overline{\mathcal{J}}$. We build a diagram $G: \mathscr{C} \rightarrow$ Kom as follows:

- For each object $a \in \mathrm{Ob}(\mathscr{C})$, let $G(a)=\left(C F\left(L_{0}, L_{1}\right), \partial_{F(a)}\right)$ be the Lagrangian intersection Floer chain complex computed with respect to the almost complex structure $F(a)$.

- Fix a sequence $\left(f_{1}, \ldots, f_{n}\right)$ of composable morphisms and a generator $[\sigma] \in I_{*}^{\otimes(n-1)}$ corresponding to some $k$-dimensional face $\sigma$ of $[0,1]^{n-1}$. There is an associated $k$ parameter family of almost complex structures $\left.F\left(f_{n}, \ldots, f_{1}\right)\right|_{\sigma}$. For each intersection point $x \in L_{0} \cap L_{1}$ define

$$
G\left(f_{n}, \ldots, f_{1}\right)(\sigma \otimes x)=\sum_{y \in L_{0} \cap L_{1}} \sum_{\substack{\phi \in \pi_{2}(x, y) \\ \mu(\phi)=1-n}} \# \mathcal{M}\left(\phi ;\left.F\left(f_{n}, \ldots, f_{1}\right)\right|_{\sigma}\right) y .
$$

Note that, while we will construct the equivariant Floer cohomology below, the chain complexes $C F\left(L_{0}, L_{1}\right)$ compute the Lagrangian intersection Floer homology: we will dualize later.

Remark 3.9. Recall that we have fixed a collection of homotopy classes $\eta$ of paths from $L_{0}$ to $L_{1}$, and by Floer complex we mean the summand generated by intersection points in the classes $\eta$. Consequently, by Hypothesis 3.2, the homotopy coherent diagram $G$ from 
Construction 3.8 is a direct sum of relatively $\mathbb{Z}$-graded diagrams in the following sense: There is a decomposition at every vertex, $G(a)=\oplus_{\mathfrak{s} \in \eta} G(a)_{(\mathfrak{s})}$, and at every map, $G\left(f_{n}, \ldots, f_{1}\right)=$ $\oplus_{\mathfrak{s} \in \eta} G\left(f_{n}, \ldots, f_{1}\right)_{(\mathfrak{s})}$. For each $\mathfrak{s} \in \eta$, each of the groups $G(a)_{(\mathfrak{s})}$ is $\mathbb{Z}$-graded so that each of the maps $G\left(f_{n}, \ldots, f_{1}\right)_{(\mathfrak{s})}$ is grading preserving; and this $\mathbb{Z}$-grading is well-defined up to an overall translation (depending on $\mathfrak{s}$ ).

Lemma 3.10. Construction 3.8 defines a homotopy coherent diagram of chain complexes.

Proof. This follows by standard arguments, by considering the ends of

$$
\bigcup_{\mu(\phi)=2-n} \# \mathcal{M}\left(\phi ;\left.F\left(f_{n}, \ldots, f_{1}\right)\right|_{\sigma}\right) .
$$

Note that bubbling of spheres or disks is precluded by Hypothesis ( $\mathrm{J}-1)$, and curves can not escape to infinity by Hypothesis (J-2).

Finally, we can turn a homotopy coherent diagram of chain complexes into a single chain complex by taking the homotopy colimit (compare [Vog73, Paragraph (5.10)]), an analogue of the direct limit which behaves well with respect to homotopies and homotopy coherence:

Definition 3.11. Given a homotopy coherent diagram $G: \mathscr{C} \rightarrow \mathrm{Kom}$, the homotopy colimit of $G$ is defined by

$$
\operatorname{hocolim} G=\bigoplus_{n \geq 0} \bigoplus_{a_{0} \stackrel{f_{1}}{\longrightarrow} \ldots \stackrel{f_{n}}{\longrightarrow} a_{n}} I_{*}^{\otimes n} \otimes G\left(a_{0}\right) / \sim
$$

where the coproduct is over $n$-tuples of composable morphisms in $\mathscr{C}$ and the case $n=0$ corresponds to the objects $a_{0} \in \mathrm{Ob}(\mathscr{C})$. The equivalence relation $\sim$ is given by

$$
\begin{aligned}
& \left(f_{n}, \ldots, f_{1} ; t_{1} \otimes \cdots \otimes t_{n} ; x\right) \\
& \quad \sim \begin{cases}\left(f_{n}, \ldots, f_{2} ; \pi\left(t_{1}\right) \otimes t_{2} \otimes \cdots \otimes t_{n} ; x\right) & f_{1}=\mathbb{I} \\
\left(f_{n}, \ldots, f_{i+1}, f_{i-1}, \ldots, f_{1} ; t_{1} \otimes \cdots \otimes m\left(t_{i-1}, t_{i}\right) \otimes \cdots \otimes t_{n} ; x\right) & f_{i}=\mathbb{I}, i>1 \\
\left(f_{n}, \ldots, f_{i+1} \circ f_{i}, \ldots, f_{1} ; t_{1} \otimes \cdots \otimes t_{i-1} \otimes t_{i+1} \otimes \cdots \otimes t_{n} ; x\right) & t_{i}=\{1\}, i<n \\
\left(f_{n-1}, \ldots, f_{1} ; t_{1} \otimes \cdots \otimes t_{n-1} ; x\right) & t_{n}=\{1\} \\
\left(f_{n}, \ldots, f_{i+1} ; t_{i+1} \otimes \cdots \otimes t_{n} ; G\left(f_{i}, \ldots, f_{1}\right)\left(t_{1} \otimes \cdots \otimes t_{i-1} \otimes x\right)\right) & t_{i}=\{0\} .\end{cases}
\end{aligned}
$$

The differential is induced by the tensor product differential in Formula (3.12), i.e., $\partial\left(\left(f_{n}, \ldots, f_{1} ; t_{1} \otimes \cdots \otimes t_{n} ; x\right)\right)=\left(f_{n}, \ldots, f_{1} ; \partial\left(t_{1} \otimes \cdots \otimes t_{n}\right) ; x\right)+\left(f_{n}, \ldots, f_{1} ; t_{1} \otimes \cdots \otimes t_{n} ; \partial(x)\right)$.

The homotopy colimit has a filtration $\mathcal{F}$ where $\mathcal{F}_{n}$ hocolim $G$ is the span of the expressions $\left(f_{m}, \ldots, f_{1} ;\{0,1\} \otimes \cdots \otimes\{0,1\} ; x\right), m \leq n$, i.e., the length $\leq n$ composable sequences with all of the $t_{i}$ the 1 -simplex $\{0,1\}$. (The special case $\mathcal{F}_{0}$ hocolim $G$ is $\bigoplus_{a \in \mathrm{Ob}(\mathscr{C})} G(a)$.)

See Example A.3 for an illustration of homotopy colimits of complexes.

The homotopy colimit hocolim $G$ of a diagram from Construction 3.8 is a relatively $\mathbb{Z}$ graded (in the sense of Remark 3.9), bounded-below chain complex. 
3.4. Building the equivariant cochain complex. Throughout this section we fix a symplectic manifold $(M, \omega)$, a symplectic involution $\tau: M \rightarrow M$, Lagrangians $L_{0}, L_{1} \subset M$ fixed by $\tau$, and a collection of homotopy classes of paths $\eta$ from $L_{0}$ to $L_{1}$, such that this data satisfies Hypothesis 3.2.

Lemma 3.13. There is a sufficiently generic homotopy coherent diagram $F: \mathscr{E} \mathbb{Z} / 2 \rightarrow \overline{\mathcal{J}}$ which is $\tau$-equivariant, i.e., so that $F(b)=\tau_{*}(F(a))=d \tau \circ F(a) \circ d \tau$ and for any sequence $\left(f_{1}, \ldots, f_{n}\right)$ of composable morphisms and any $\left(t_{1}, \ldots, t_{n-1}\right) \in[0,1]^{n-1}$,

$$
\tau_{*}\left(F\left(f_{n}, \ldots, f_{1}\right)\left(t_{1}, \ldots, t_{n-1}\right)\right)=F\left(\tau\left(f_{n}\right), \ldots, \tau\left(f_{1}\right)\right)\left(t_{1}, \ldots, t_{n-1}\right) .
$$

Proof. The construction of the $F\left(f_{n}, \ldots, f_{1}\right)$ is inductive in $n$, using the facts that $\overline{\mathcal{J}}$ is weakly contractible; Lemma 3.6; and the fact that the action of $\tau$ on $\mathscr{E} \mathbb{Z} / 2$ (and hence on sequences of morphisms in $\mathscr{E} \mathbb{Z} / 2$ ) is free. Details are left to the reader.

The equivariance condition implies that for any sequence $(\alpha, \beta, \ldots)$, say, of composable morphisms in $\mathscr{E}$ and any $x \in C F\left(L_{0}, L_{1}\right)=G(a)$,

$$
G_{\ldots, \beta, \alpha}(x)=\tau_{\#}\left(G_{\ldots, \alpha, \beta}\left(\tau_{\#}(x)\right)\right),
$$

where $\tau_{\#}: C F\left(L_{0}, L_{1}\right) \rightarrow C F\left(L_{0}, L_{1}\right)$ is the map induced by $\tau$ (i.e., takes a point $x \in L_{0} \cap L_{1}$ to $\tau(x)$ ). (The map $\tau_{\#}$ is a chain map from $C F\left(L_{0}, L_{1} ; \partial_{F(a)}\right)$ to $C F\left(L_{0}, L_{1} ; \partial_{F(b)}\right)$.)

Lemma 3.14. For any sufficiently generic diagram $F: \mathscr{E} \mathbb{Z} / 2 \rightarrow \overline{\mathcal{J}}$ with associated diagram $G: \mathscr{E} \mathbb{Z} / 2 \rightarrow$ Kom as in Construction 3.8, the complex hocolim $G$ is quasi-isomorphic to $G(a)=\left(C F\left(L_{0}, L_{1}\right), \partial_{F(a)}\right)$.

Proof. Since $a$ is a terminal object of $\mathscr{E} \mathbb{Z} / 2$, hocolim $\mathscr{E} \mathbb{Z} / 2 G \simeq G(a)$ [BK72, Example XII.3.1]. (One can also give a concrete proof using the explicit description of hocolim $\mathscr{E}_{\mathbb{Z} / 2} G$ from Observation 3.16, below.)

Definition 3.15. Fix a $\tau$-equivariant, sufficiently generic $F: \mathscr{E} \mathbb{Z} / 2 \rightarrow \overline{\mathcal{J}}$ and let $G: \mathscr{E} \mathbb{Z} / 2 \rightarrow$ Kom be as in Construction 3.8. We define the freed Floer complex of $\left(L_{0}, L_{1}\right)$ to be

$$
\widetilde{C F}\left(L_{0}, L_{1}\right):=\operatorname{hocolim} G \text {. }
$$

By Lemma 3.14, $\widetilde{C F}\left(L_{0}, L_{1}\right)$ is quasi-isomorphic, over $\mathbb{F}_{2}$, to $C F\left(L_{0}, L_{1}\right)$. The action of $\mathbb{Z} / 2$ on $\mathscr{E} \mathbb{Z} / 2$ makes $\widetilde{C F}\left(L_{0}, L_{1}\right)$ into a chain complex of free modules over $\mathbb{F}_{2}[\mathbb{Z} / 2]$. The equivariant Floer cochain complex of $\left(L_{0}, L_{1}\right)$ is the complex

$$
C F_{\mathbb{Z} / 2}\left(L_{0}, L_{1}\right):=\operatorname{Hom}_{\mathbb{F}_{2}[\mathbb{Z} / 2]}\left(\operatorname{hocolim} G, \mathbb{F}_{2}\right) \text {. }
$$

Let $H F_{\mathbb{Z} / 2}\left(L_{0}, L_{1}\right)$ be the homology of $C F_{\mathbb{Z} / 2}\left(L_{0}, L_{1}\right)$, i.e., the equivariant Floer cohomology of $\left(M, L_{0}, L_{1}, \tau\right)$. Since the complex $\widetilde{C F}\left(L_{0}, L_{1}\right)$ is bounded below and free over $\mathbb{F}_{2}[\mathbb{Z} / 2]$, so $H F_{\mathbb{Z} / 2}\left(L_{0}, L_{1}\right)$ is the same as $\operatorname{Ext}_{\mathbb{F}_{2}[\mathbb{Z} / 2]}\left(\right.$ hocolim $\left.G, \mathbb{F}_{2}\right)$.

The filtration $\mathcal{F}$ on hocolim $G$ is preserved by the $\mathbb{F}_{2}[\mathbb{Z} / 2]$-action, and hence induces a filtration on $C F_{\mathbb{Z} / 2}\left(L_{0}, L_{1}\right)$, which we also denote $\mathcal{F}$.

Observation 3.16. In Section 4 we will need a somewhat more concrete description of parts of the complexes $\widetilde{C F}\left(L_{0}, L_{1}\right)$ and $C F_{\mathbb{Z} / 2}\left(L_{0}, L_{1}\right)$. So, fix diagrams $F$ and $G$ as in Definition 3.15. The homotopy colimit hocolim $G=\widetilde{C F}\left(L_{0}, L_{1}\right)$ has a basis consisting of elements of the form $\left(f_{n}, \ldots, f_{1} ;\{0,1\} \otimes \cdots \otimes\{0,1\} ; x\right)$ where $x \in L_{0} \cap L_{1}$ and $\left(f_{1}, \ldots, f_{n}\right)$ is a sequence of composable, non-identity elements. Thus, the elements have the form $\alpha_{n} \otimes x:=(\alpha, \beta, \alpha, \beta, \ldots ;\{0,1\}, \ldots,\{0,1\} ; x)$ or $\beta_{n} \otimes x:=(\beta, \alpha, \beta, \alpha, \ldots ;\{0,1\}, \ldots,\{0,1\} ; x)$ 
where the string of $\alpha$ 's and $\beta$ 's has length $n \geq 0$. The differential is given by

$$
\begin{aligned}
& \partial\left(\alpha_{n} \otimes x\right)=\alpha_{n} \otimes(\partial x)+\beta_{n-1} \otimes x+\sum_{i=1}^{n} \alpha_{n-i} \otimes\left\{\begin{array}{l}
G_{\beta, \alpha, \ldots}(x) \quad i \equiv n+1 \quad(\bmod 2) \\
G_{\alpha, \beta, \ldots}(x) i \equiv n \quad(\bmod 2)
\end{array}\right. \\
& \partial\left(\beta_{n} \otimes x\right)=\beta_{n} \otimes(\partial x)+\alpha_{n-1} \otimes x+\sum_{i=1}^{n} \beta_{n-i} \otimes\left\{\begin{array}{l}
G_{\alpha, \beta, \ldots}(x) \quad i \equiv n+1 \quad(\bmod 2) \\
G_{\beta, \alpha, \ldots}(x) \quad i \equiv n \quad(\bmod 2)
\end{array}\right.
\end{aligned}
$$

where the sequence of morphisms appearing in $G_{\alpha, \beta, \ldots}$ or $G_{\beta, \alpha, \ldots}$ in the $i^{\text {th }}$ term has length $i$. The second term in $\partial\left(\alpha_{n} \otimes x\right)$ come from the fourth case in the definition of $\sim$. The third term in $\partial\left(\alpha_{n} \otimes x\right)$ comes from the last case in the definition of $\sim$. The expression $\partial x$ means the differential with respect to the complex structure $F(a)$ or $F(b)$, depending on whether $x \in G(a)$ or $G(b)$. The $\mathbb{Z} / 2$-action exchanges $\alpha_{n} \otimes x$ and $\beta_{n} \otimes \tau_{\#}(x)$.

Passing to $C F_{\mathbb{Z} / 2}\left(L_{0}, L_{1}\right)$ dualizes the complex and identifies the elements $\left(\alpha_{n} \otimes x\right)^{*}$ and $\left(\beta_{n} \otimes \tau_{\#}(x)\right)^{*}$. We can also write $\left(\alpha_{n} \otimes x\right)^{*}$ as $\alpha_{n}^{*} \otimes x^{*}$; note that there is a Floer cochain differential $d\left(x^{*}\right)$, the dual (over $\mathbb{Z}$ ) to the differential on the Floer chain complex, and a cochain element $\tau^{\#}\left(x^{*}\right)=x^{*} \circ \tau_{\#}$. The differential on the equivariant cochain complex is given by

$$
d\left(\alpha_{n}^{*} \otimes x^{*}\right)=\alpha_{n}^{*} \otimes\left(d x^{*}\right)+\alpha_{n+1}^{*} \otimes \tau^{\#}\left(x^{*}\right)+\sum_{i=1}^{\infty} \alpha_{n+i}^{*} \otimes\left\{\begin{array}{lll}
x^{*} \circ G_{\beta, \alpha, \ldots} & n \equiv 1 & (\bmod 2) \\
x^{*} \circ G_{\alpha, \beta, \ldots} & n \equiv 0 & (\bmod 2) .
\end{array}\right.
$$

The fact that $C F\left(L_{0}, L_{1}\right)$ is finite-dimensional and $\mathbb{Z}$-graded implies that the sum in Formula (3.19) is finite.

There is an action of $\mathbb{F}_{2}[\theta]$ on $C F_{\mathbb{Z} / 2}\left(L_{0}, L_{1}\right)$ by $\theta \cdot\left(\alpha_{n}^{*} \otimes x^{*}\right)=\alpha_{n+1}^{*} \otimes \tau^{\#}\left(x^{*}\right)=\beta_{n+1}^{*} \otimes x^{*}$.

Lemma 3.20. The homology of the associated graded complex to $\left(C F_{\mathbb{Z} / 2}\left(L_{0}, L_{1}\right), \mathcal{F}\right)$ is given by $H F^{*}\left(L_{0}, L_{1}\right) \otimes_{\mathbb{F}_{2}} \mathbb{F}_{2}[\theta]$. In particular, there is a spectral sequence $H F^{*}\left(L_{0}, L_{1}\right) \otimes_{\mathbb{F}_{2}} \mathbb{F}_{2}[\theta] \Rightarrow$ $H F_{\mathbb{Z} / 2}\left(L_{0}, L_{1}\right)$ from the Floer cohomology of $\left(L_{0}, L_{1}\right)$ tensored with $\mathbb{F}_{2}[\theta]$ to the equivariant Floer cohomology. Further, both this spectral sequence and the module structure over $\mathbb{F}_{2}[\theta]$ agree with the spectral sequence and module structure described in Section 2 for the chain complex $\widetilde{C F}\left(L_{0}, L_{1}\right)$ over $\mathbb{F}_{2}[\mathbb{Z} / 2]$.

Proof. The explicit description in Observation 3.16 implies the spectral sequence has the specified form: we have $\mathcal{F}\left(\alpha_{n}^{*} \otimes x^{*}\right)=n$, and the only term of $d\left(\alpha_{n}^{*} \otimes x^{*}\right)$ in filtration $n$ is $\alpha_{n}^{*} \otimes\left(d x^{*}\right)$.

To see that this agrees with the spectral sequence in Section 2, first note that the homotopy coherent diagram $G$ is equivalent to a strict diagram $\widetilde{G}: \mathscr{E} \mathbb{Z} / 2 \rightarrow \mathrm{Kom}$. That is, there is a strict diagram $\widetilde{G}: \mathscr{E} \mathbb{Z} / 2 \rightarrow$ Kom and a homotopy coherent diagram $H: \mathscr{I} \times \mathscr{E} \mathbb{Z} / 2 \rightarrow \mathrm{Kom}$, where $\mathscr{I}=(0 \longrightarrow 1)$, so that $\left.H\right|_{\{1\} \times \mathscr{E} \mathbb{Z} / 2}=\widetilde{G}$ and $\left.H\right|_{\{0\} \times \mathscr{E} \mathbb{Z} / 2}=G$ [CP86, Corollary 4.5]. Further, it follows from the construction that we can arrange for $\widetilde{G}$ and $H$ to be $\mathbb{Z} / 2$ equivariant. Associated to $H$, then, is a quasi-isomorphism $\bar{H}: \operatorname{hocolim} G \rightarrow \operatorname{hocolim} \widetilde{G}$ of complexes over $\mathbb{F}_{2}[\mathbb{Z} / 2]$. It follows from the construction of $\bar{H}$ that it induces a filtered, $\mathbb{F}_{2}[\theta]$-equivariant map $\bar{H}^{*}: \operatorname{Hom}_{\mathbb{F}_{2}[\mathbb{Z} / 2]}\left(\operatorname{hocolim} \widetilde{G}, \mathbb{F}_{2}\right) \rightarrow \operatorname{Hom}_{\mathbb{F}_{2}[\mathbb{Z} / 2]}\left(\operatorname{hocolim} G, \mathbb{F}_{2}\right)$. (The differential on hocolim $\widetilde{G}$ is given by a formula analogous to Equation (3.19), with the simplification that the sum over $i$ only involves the single term $i=1$.) 
Thus, it suffices to prove the result when $G$ is a strict diagram. In this case, the complex hocolim $G$ is given by

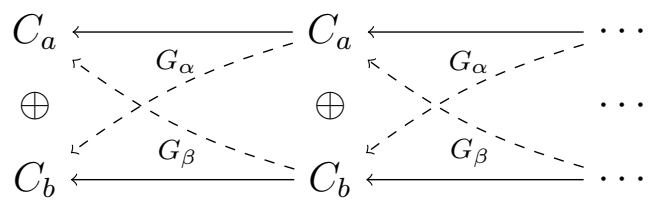

where $C_{a}=G(a), C_{b}=G(b)$, and all solid arrows are the identity map. (We have not drawn the internal differentials of $C_{a}$ and $C_{b}$.) The complex $C F_{\mathbb{Z} / 2}\left(L_{0}, L_{1}\right)$ is, thus,

$$
0 \longrightarrow C_{a}^{*} \stackrel{\mathbb{I}+\tau^{\#} \circ G_{\beta}^{*}}{\longrightarrow} C_{a}^{*} \stackrel{\mathbb{I}+\tau^{\#} \circ G_{\beta}^{*}}{\longrightarrow} \cdots
$$

This complex is filtered by the columns, and the action of $\theta$ moves elements one step to the right. (In the notation of Observation 3.16, the copy of $C_{a}^{*}$ in the $n$th filtration level is generated by the elements $\left\{\beta_{n}^{*} \otimes x^{*}\right\}$, for $x \in L_{0} \cap L_{1}$, if $n$ is even, and $\left\{\alpha_{n}^{*} \otimes x^{*}\right\}$ if $n$ is odd.) By contrast, tensoring hocolim $G$ with the standard resolution of the $\mathbb{F}_{2}[\mathbb{Z} / 2]$-module $\mathbb{F}_{2}$ (Section 2) gives

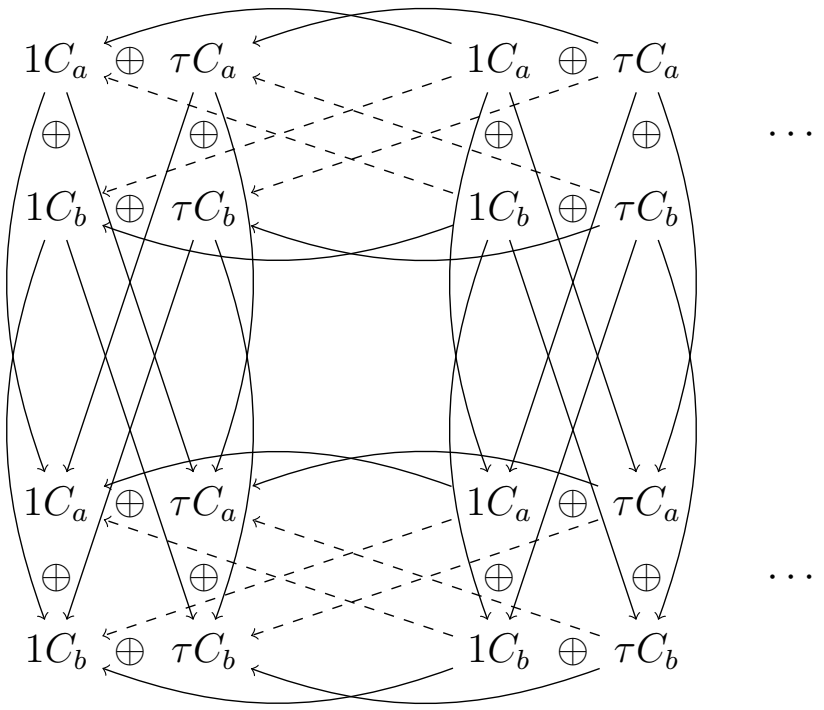

Here, we have identified $\mathbb{F}_{2}[\mathbb{Z} / 2]=\mathbb{F}_{2}[\tau] /\left(\tau^{2}=1\right)$ and written, e.g., $\tau C_{a}$ for $\tau \otimes C_{a}$. The action of $\tau$ exchanges $1 C_{a}$ and $\tau C_{b}$ and exchanges $1 C_{b}$ and $\tau C_{a}$ in each block. Solid arrows are, again, identity maps, while dashed arrows are $G_{\alpha}$ or $G_{\beta}$, depending on whether the source is $C_{a}$ or $C_{b}$. 
Taking Hom over $\mathbb{F}_{2}[\mathbb{Z} / 2]$ to $\mathbb{F}_{2}$ gives

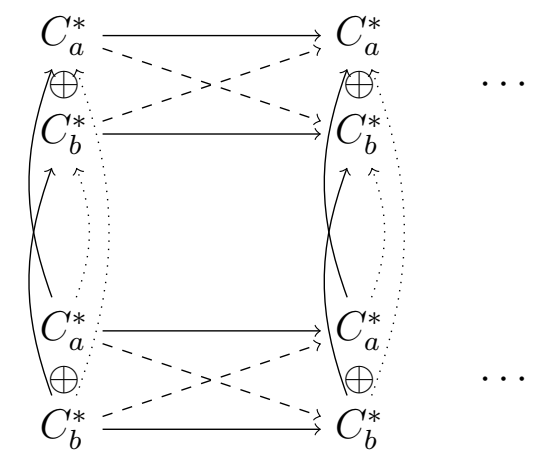

where the solid arrows are again identity maps and the dashed arrows are $G_{\alpha}^{*}$ or $G_{\beta}^{*}$, depending on whether the source is $C_{b}^{*}$ or $C_{a}^{*}$. Dotted arrows indicate the map $\tau^{\#}$. There is a quasi-isomorphism from the complex (3.21) to the complex (3.22), given by

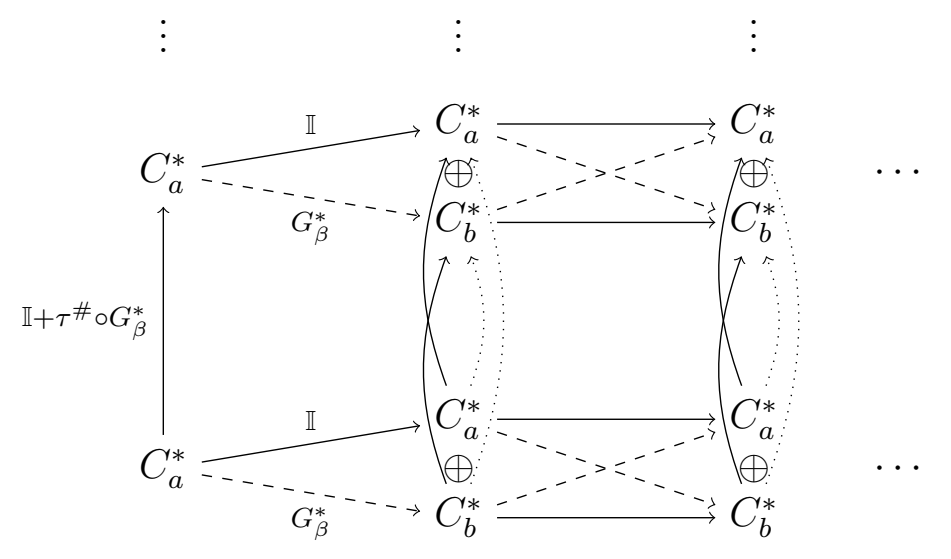

To see that this map is an isomorphism, filter by the $y$-coordinate and note that the map induces an isomorphism on the $E^{1}$-page of the associated spectral sequence. The quasiisomorphism intertwines the actions of $\mathbb{F}_{2}[\theta]$ and the filtrations, proving the result.

\subsection{Invariance.}

Proposition 3.23. Up to quasi-isomorphism of complexes over $\mathbb{F}_{2}[\mathbb{Z} / 2]$, the freed Floer complex $\widetilde{C F}\left(L_{0}, L_{1}\right)$ is independent of the choice of $\tau$-equivariant sufficiently generic homotopy coherent diagram in $\overline{\mathcal{J}}$ used to define it.

As discussed in Section 2, it follows that $C F_{\mathbb{Z} / 2}\left(L_{0}, L_{1}\right)$ is well-defined up to filtered quasiisomorphism, and that $H F_{\mathbb{Z} / 2}\left(L_{0}, L_{1}\right)$ is well-defined up to isomorphism over $\mathbb{F}_{2}[\theta]$.

Proof. Let $\mathscr{I}$ be the category with two objects, 0 and 1 , and a single morphism $f_{0,1}: 0 \rightarrow 1$ :

$$
\mathscr{I}=\mathbb{I} C 0 \stackrel{f_{0,1}}{\longrightarrow} 1 \bigcirc \mathbb{I} .
$$

Given homotopy coherent $\mathscr{C}$-diagrams $G_{0}$ and $G_{1}$ in $\overline{\mathcal{J}}$ (respectively Kom), a morphism from $G_{0}$ to $G_{1}$ is a homotopy coherent $\mathscr{I} \times \mathscr{C}$-diagram $H$ in $\overline{\mathcal{J}}$ (respectively Kom) such 
that $\left.H\right|_{\{i\} \times \mathscr{C}}=G_{i}$ for $i=0,1$. For a morphism $H$ of diagrams in Kom, we will call $H$ a quasi-isomorphism if

$$
H\left(f_{0,1} \times \mathbb{I}_{x}\right): G_{0}(x) \rightarrow G_{1}(x)
$$

is a quasi-isomorphism. It is not hard to show that morphisms of diagrams induce morphisms of homotopy colimits and that if $H$ is a quasi-isomorphism then the induced map hocolim $H$ : hocolim $G_{0} \rightarrow$ hocolim $G_{1}$ is a quasi-isomorphism. (See Vogt [Vog73, Proposition (4.6) and Theorem (5.12)] for the analogous result in the topological case and Cordier-Porter [CP86] for a setting which includes chain complexes.)

Now, fix sufficiently generic, equivariant $\mathscr{E} \mathbb{Z} / 2$-diagrams $F_{0}$ and $F_{1}$ in $\overline{\mathcal{J}}$, and let $G_{0}, G_{1}$ : $\mathscr{E} \mathbb{Z} / 2 \rightarrow$ Kom be the maps given by Construction 3.8. By the same argument as in the proof of Lemma 3.13, we can extend $F_{0}$ and $F_{1}$ to a diagram $H: \mathscr{I} \times \mathscr{E} \mathbb{Z} / 2 \rightarrow \overline{\mathcal{J}}$ which is $\tau$-equivariant (where $\tau$ acts trivially on $\mathscr{I}$ ). Applying Construction 3.8 to $H$ gives a diagram $K: \mathscr{I} \times \mathscr{E} \mathbb{Z} / 2 \rightarrow$ Kom.

In any diagram $G: \mathscr{C} \rightarrow$ Kom obtained from Construction 3.8, all edges map to quasiisomorphisms: an edge is sent to a Floer continuation map associated to a (sufficiently generic) path of almost complex structures. In particular, the diagram $K$ is a quasiisomorphism from $G_{0}$ to $G_{1}$. So, as above, $K$ induces a quasi-isomorphism

$$
\operatorname{hocolim} K: \operatorname{hocolim} G_{0} \rightarrow \operatorname{hocolim} G_{1}
$$

and, since $H$ is $\mathbb{Z} / 2$-equivariant, hocolim $K$ is a map of $\mathbb{F}_{2}[\mathbb{Z} / 2]$-modules.

We turn next to isotopy invariance. First, recall that we fixed a $\mathbb{Z} / 2$-invariant collection $\eta$ of homotopy classes of paths from $L_{0}$ to $L_{1}$, and $\widetilde{C F}\left(L_{0}, L_{1}\right)$ is defined using only intersection points that belong to these homotopy classes. Given an isotopy from $L_{0}$ (respectively $L_{1}$ ) to another Lagrangian $L_{0}^{\prime}$ (respectively $L_{1}^{\prime}$ ), the homotopy classes $\eta$ induces a collection of homotopy classes of paths $\eta^{\prime}$ from $L_{0}^{\prime}$ to $L_{1}^{\prime}$. If $\eta$ is preserved by the $\mathbb{Z} / 2$-action and the isotopies are $\mathbb{Z} / 2$-equivariant then $\eta^{\prime}$ is preserved by the $\mathbb{Z} / 2$-action, as well.

Proposition 3.24. If $L_{0}^{\prime}$ and $L_{1}^{\prime}$ are isotopic to $L_{0}$ and $L_{1}$ via compactly-supported, Hamiltonian isotopies through $\tau$-invariant Lagrangians then there is a quasi-isomorphism of complexes $\widetilde{C F}\left(L_{0}, L_{1}\right) \simeq \widetilde{C F}\left(L_{0}^{\prime}, L_{1}^{\prime}\right)$ over $\mathbb{F}_{2}[\mathbb{Z} / 2]$. (Here, the $\widetilde{C F}\left(L_{0}, L_{1}\right)$ and $\widetilde{C F}\left(L_{0}^{\prime}, L_{1}^{\prime}\right)$ are defined using corresponding collections of homotopy classes of paths $\eta$ and $\eta^{\prime}$, respectively.)

Proof. The proof is essentially the same as the proof of Proposition 3.23, but using the Hamiltonian isotopy instead of a path of almost complex structures to define the map between diagrams.

If one of the triple intersections of $L_{0}, L_{1}, L_{0}^{\prime}, L_{1}^{\prime}$ is non-empty (i.e., the Lagrangians are not triple-wise transverse) there is an additional step, so assume first that all triple intersections are empty. Consider the topological category $\mathscr{D}$ where:

- The objects of $\mathscr{D}$ are $\mathrm{Ob}(\overline{\mathcal{J}}) \amalg \mathrm{Ob}(\overline{\mathcal{J}})=\{0,1\} \times \mathrm{Ob}(\overline{\mathcal{J}})$.

- For $(i, J),\left(i, J^{\prime}\right) \in\{i\} \times \operatorname{Ob}(\overline{\mathcal{J}}), \operatorname{Hom}_{\mathscr{D}}\left((i, J),\left(i, J^{\prime}\right)\right)=\operatorname{Hom}_{\overline{\mathcal{J}}}\left(J, J^{\prime}\right)$ is the space of sequences of eventually cylindrical almost complex structures for $J$ to $J^{\prime}$.

- $\operatorname{Hom}_{\mathscr{D}}\left((1, J),\left(0, J^{\prime}\right)\right)=\varnothing$.

- $\operatorname{Hom}_{\mathscr{D}}\left((0, J),\left(1, J^{\prime}\right)\right)$ is given by sequences $\left(\widetilde{J}_{-i}, \ldots, \widetilde{J}_{-1}, \widetilde{J}_{0}, \widetilde{J}_{1}, \ldots, \widetilde{J}_{j}\right)$ of eventually cylindrical almost complex structures with one distinguished level, $\widetilde{J}_{0}$ where we do not quotient by $\mathbb{R}$-translation. (The level $\widetilde{J}_{0}$ is where the Hamiltonian isotopy will 
occur.) There is a quotient map $\operatorname{Hom}_{\mathscr{D}}\left((0, J),\left(1, J^{\prime}\right)\right) \rightarrow \operatorname{Hom}_{\overline{\mathcal{J}}}\left(J, J^{\prime}\right)$, by modding out by $\mathbb{R}$ on the distinguished level.

Given a homotopy coherent diagram $\mathscr{C} \rightarrow \mathscr{D}$, we can apply Floer theory to obtain a diagram $G: \mathscr{C} \rightarrow$ Kom as follows. Given an object $x$ of $\mathscr{C}$, with $F(x)=(i, J)$, let

$$
G(x)= \begin{cases}C F\left(L_{0}, L_{1} ; J\right) & i=0 \\ C F\left(L_{0}^{\prime}, L_{1}^{\prime} ; J\right) & i=1\end{cases}
$$

Given a morphism $f$ in $\mathscr{C}$, if $F(f)=\widetilde{J}$ is contained in $\{0\} \times \overline{\mathcal{J}}$ (respectively $\{1\} \times \overline{\mathcal{J}}$ ) then define $G(f)$ to be the continuation map on $C F\left(L_{0}, L_{1}\right)$ (respectively $C F\left(L_{0}^{\prime}, L_{1}^{\prime}\right)$ ) associated to $\widetilde{J}$. If $F(f)=\left(\widetilde{J}_{-i}, \ldots, \widetilde{J}_{-1}, \widetilde{J}_{0}, \widetilde{J}_{1}, \ldots, \widetilde{J}_{j}\right)$ maps from $(0, J)$ to $\left(1, J^{\prime}\right)$ then define $G(f)$ to be the composition of the continuation maps associated to the $J_{k}, k=-i, \ldots,-1$, on $C F\left(L_{0}, L_{1}\right)$, followed by the continuation map associated to the Hamiltonian isotopy from $\left(L_{0}, L_{1}\right)$ to $\left(L_{0}^{\prime}, L_{1}^{\prime}\right)$ computed with respect to the almost complex structure $\widetilde{J}_{0}$, and then followed by the continuation maps associated to the $\widetilde{J}_{k}, k=1, \ldots, j$, on $C F\left(L_{0}^{\prime}, L_{1}^{\prime}\right)$. More generally, given a sequence of morphisms $f_{1}, \ldots, f_{n}$, define $G\left(f_{n}, \ldots, f_{1}\right)$ to be as in Construction 3.8 if all of the $f_{i}$ are in $\{0\} \times \overline{\mathcal{J}}$ or $\{1\} \times \overline{\mathcal{J}}$, and using the Hamiltonian isotopy at the 0-level together with the family of almost complex structures $F\left(f_{n}, \ldots, f_{1}\right)$ to obtain a map $C F\left(L_{0}, L_{1}\right) \rightarrow C F\left(L_{0}^{\prime}, L_{1}^{\prime}\right)$ if $f_{n} \circ \cdots \circ f_{1}$ maps from $\{0\} \times \overline{\mathcal{J}}$ to $\{1\} \times \overline{\mathcal{J}}$. It is clear that for generically chosen functors $F: \mathscr{C} \rightarrow \mathscr{D}, G$ defines a homotopy coherent diagram of complexes.

Observe that the $\mathbb{Z} / 2$-action on $(M, \omega)$ induces a $\mathbb{Z} / 2$-action on $\mathscr{D}$. Given $\tau$-equivariant homotopy coherent functors $F, F^{\prime}: \mathscr{E} \mathbb{Z} / 2 \rightarrow \overline{\mathcal{J}}$ used to define $\widetilde{C F}\left(L_{0}, L_{1}\right)$ and $\widetilde{C F}\left(L_{0}^{\prime}, L_{1}^{\prime}\right)$, respectively, there is no obstruction to extending $\{0\} \times F \amalg\{1\} \times F^{\prime}$ to a $\tau$-equivariant homotopy coherent functor $F^{\prime \prime}: \mathscr{I} \times \mathscr{E} \mathbb{Z} / 2 \rightarrow \mathscr{D}$. The construction above then gives a homotopy coherent diagram $G^{\prime \prime}: \mathscr{I} \times \mathscr{E} \mathbb{Z} / 2 \rightarrow$ Kom. On each edge $f_{0,1} \times \mathbb{I}_{x}, G^{\prime \prime}$ is induced by a continuation map, and hence is a quasi-isomorphism. So, as in the proof of Proposition 3.23, $G^{\prime \prime}$ induces a quasi-isomorphism $\widetilde{C F}\left(L_{0}, L_{1}\right)=\operatorname{hocolim} G \rightarrow \operatorname{hocolim} G^{\prime}=\widetilde{C F}\left(L_{0}^{\prime}, L_{1}^{\prime}\right)$ over $\mathbb{F}_{2}[\mathbb{Z} / 2]$.

This proves the result, under the assumption of no triple intersections. In the case of triple intersections there may be constant bigons contained in $L_{0} \cap L_{0}^{\prime} \cap L_{1}$, say, which are not transversely cut out by the $\bar{\partial}$-operator for any almost complex structure. (By considering different twistings of the diagrams in Section 6.1, it is easy to construct Lagrangians in which such disks exist.) We discuss how to handle this case in more detail in Section 3.6. Briefly, extend the category $\mathscr{D}$ above to include small Hamiltonian perturbations of $L_{1}$ along $\{1\} \times \mathbb{R} \subset \partial \mathbb{D}^{2}$ in the morphisms from $(0, J)$ to $\left(1, J^{\prime}\right)$. With this larger space of perturbations we are able to achieve transversality for all homotopy classes of triangles, and the proof proceeds as before.

In fact, under certain circumstances we do not need the isotopy to be through equivariant Lagrangians. Recall that objects $L$ and $L^{\prime}$ in an $A_{\infty}$-category (such as the Fukaya category) are homotopy equivalent if there are degree-zero cycles $f \in \operatorname{Hom}\left(L, L^{\prime}\right)$ and $g \in \operatorname{Hom}\left(L^{\prime}, L\right)$ such that the compositions $\circ_{2}(g, f) \in \operatorname{Hom}(L, L)$ and $\circ_{2}(f, g) \in \operatorname{Hom}\left(L^{\prime}, L^{\prime}\right)$ are homotopic to the identity maps of $L$ and $L^{\prime}$, respectively. 
Proposition 3.25. Suppose that $L_{0}, L_{0}^{\prime}$ and $L_{1}$ are $\tau$-invariant Lagrangians, which are pairwise transverse, and that $L_{0}$ is Hamiltonian isotopic to $L_{0}^{\prime}$ or, more generally, so that $L_{0}$ is homotopy equivalent to $L_{1}$ in the Fukaya category. Suppose further that:

(1) The collection of homotopy classes $\eta^{\prime}$ induced by $\eta$ and the Hamiltonian isotopies is preserved by the $\mathbb{Z} / 2$-action. (Of course, $\eta$ is also required to be preserved by the $\mathbb{Z} / 2$-action, per Hypothesis 3.2.)

(2) There is a $\tau$-invariant, $\omega$-compatible almost complex structure $J$ on $M$ which achieves transversality for all moduli spaces of holomorphic bigons with boundary on $\left(L_{0}, L_{0}^{\prime}\right)$ of Maslov index $\leq 1$.

(3) The top class in $H F\left(L_{0}, L_{0}^{\prime}\right)$ (i.e., the isomorphism between $L_{0}$ and $L_{0}^{\prime}$ in the Fukaya category) is represented by a cycle $1 \in C F\left(L_{0}, L_{0}^{\prime}\right)$ which is $\tau$-invariant, $\tau_{\#}(1)=1$.

Then there is a quasi-isomorphism $\widetilde{C F}\left(L_{0}, L_{1}\right) \simeq \widetilde{C F}\left(L_{0}^{\prime}, L_{1}\right)$ of complexes over $\mathbb{F}_{2}[\mathbb{Z} / 2]$.

Proof. Assume first that the triple intersection $L_{0} \cap L_{0}^{\prime} \cap L_{1}$ is empty. In this case, the proof is similar to the proof of Proposition 3.24, but with the category $\mathscr{D}$ replaced by a different target category. Specifically, let $\Delta$ be a disk with three boundary punctures, labeled $p_{1}, p_{2}$, $p_{3}$ in clockwise order (i.e., a triangle with corners $p_{1}, p_{2}$ and $p_{3}$ ). Fix an identification of a small, closed neighborhood of $p_{i}$ with $[0, \infty) \times[0,1]$. Fix also a $\tau$-invariant cylindrical almost complex structure $J$ on $M$ achieving transversality for $\left(L_{0}, L_{0}^{\prime}\right)$. Let $\mathcal{J}_{\Delta}$ denote the space of almost complex structures on $M$ parameterized by $\Delta$ which are translation-invariant near the punctures of $\Delta$, and which agree with $J$ near $p_{1}$. That is, $\mathcal{J}_{\Delta}$ is the space of maps $\widetilde{J}_{\Delta}$ from $\Delta$ to the space of almost complex structures on $M$ compatible with $\omega$ so that for each of the punctures of $\Delta$ there is an $N$ with $\left.\widetilde{J}_{\Delta}\right|_{[N, \infty) \times[0,1]}$ translation-invariant in the first factor, and so that on the specified neighborhood $[0, \infty) \times[0,1]$ of $p_{1}, \widetilde{J}_{\Delta}=J$. Define a category $\mathscr{D}$ with:

- $\mathrm{Ob}(\mathscr{D})=\mathrm{Ob}(\overline{\mathcal{J}}) \amalg \mathrm{Ob}(\overline{\mathcal{J}})=\{0,1\} \times \mathrm{Ob}(\overline{\mathcal{J}})$.

- For $(i, J),\left(i, J^{\prime}\right) \in\{i\} \times \operatorname{Ob}(\overline{\mathcal{J}}), \operatorname{Hom}_{\mathscr{D}}\left((i, J),\left(i, J^{\prime}\right)\right)=\operatorname{Hom}_{\overline{\mathcal{J}}}\left(J, J^{\prime}\right)$ is the space of sequences of eventually cylindrical almost complex structures for $J$ to $J^{\prime}$.

- $\operatorname{Hom}\left((0, J),\left(1, J^{\prime}\right)\right)$ is given by sequences $\left(\widetilde{J}_{-i}, \ldots, \widetilde{J}_{-1}, \widetilde{J}_{0}, \widetilde{J}_{1}, \ldots, \widetilde{J}_{j}\right)$ where:

- For $k \neq 0$, each $\widetilde{J}_{k} \in \mathcal{J}\left(J_{k}, J_{k+1}\right)$ is a eventually cylindrical almost complex structure. Here, $J_{-i}, \ldots, J_{j+1}$ is some sequence of cylindrical almost complex structures.

$-J_{-i}=J$ and $J_{j+1}=J^{\prime}$.

$-\widetilde{J}_{0} \in \mathcal{J}_{\Delta}$ is a family of almost complex structures parameterized by $\Delta$ which agree with $J_{0}$ on some cylindrical neighborhood $[n, \infty) \times[0,1]$ of $p_{2}, J_{1}$ on some cylindrical neighborhood $[n, \infty) \times[0,1]$ of $p_{3}$ and, of course, $J$ on some cylindrical neighborhood $[n, \infty) \times[0,1]$ of $p_{1}$.

Like $\overline{\mathcal{J}}, \mathscr{D}$ is a topological category.

Let $1 \in C F\left(L_{0}, L_{0}^{\prime} ; J\right)$ be the $\tau$-invariant homotopy equivalence guaranteed by the proposition's hypotheses, and write 1 as a sum of intersection points between $L_{0}$ and $L_{0}^{\prime}, 1=\sum_{i} 1_{i}$ with $1_{i} \in L_{0} \cap L_{0}^{\prime}$. Fix a complex structure $j_{\Delta}$ on $\Delta$ so that the identifications of the neighborhoods of the punctures with $[0, \infty) \times[0,1]$ are $j_{\Delta}$-holomorphic. Given a complex structure $\widetilde{J} \in \mathcal{J}_{\Delta}$ and points $x \in L_{0} \cap L_{1}$ and $y \in L_{0}^{\prime} \cap L_{1}$ we can consider the moduli space $\mathcal{M}\left(x, y, 1_{i} ; \widetilde{J}\right)$ of holomorphic sections of the bundle $\left(M \times \Delta, \widetilde{J} \times j_{\Delta}\right) \rightarrow\left(\Delta, j_{\Delta}\right)$ which send the three edges of the triangle to $L_{0}^{\prime}, L_{0}$, and $L_{1}$ and are asymptotic to $1_{i}$ 
at $p_{1}, x$ at $p_{2}$, and $y$ at $p_{3}$. The space $\mathcal{M}\left(x, y, 1_{i} ; \widetilde{J}\right)$ decomposes according to homotopy classes of triangles, $\mathcal{M}\left(x, y, 1_{i} ; \widetilde{J}\right)=\amalg_{\phi \in \pi_{2}\left(x, y, 1_{i}\right)} \mathcal{M}(\phi ; \widetilde{J})$. More generally, given a family of complex structures $\widetilde{J}:[0,1]^{\ell} \rightarrow \mathcal{J}_{\Delta}$ and a homotopy class of triangles $\phi$ we can consider the moduli space $\mathcal{M}(\phi ; \widetilde{J})=\cup_{\vec{t} \in[0,1]} \mathcal{M}(\phi ; \widetilde{J}(\vec{t}))$. For generic families $\widetilde{J}$, these moduli spaces are transversely cut out. Given a generic family $\widetilde{J}$ there is a corresponding map $G(\widetilde{J}): C F\left(L_{0}, L_{1} ; J_{0}\right) \rightarrow C F\left(L_{0}^{\prime}, L_{1} ; J_{1}\right)$ defined by

$$
G(x)=\sum_{y \in L_{0}^{\prime} \cap L_{1}} \sum_{1_{i} \in 1} \sum_{\substack{\phi \in \pi_{2}\left(x, y, 1_{i}\right) \\ \mu(\phi)=-\ell}} \# \mathcal{M}(\phi ; \widetilde{J}) y .
$$

If $\ell=0$ then $G(\widetilde{J})$ is a chain map and, in fact, a chain homotopy equivalence.

Given a sufficiently generic homotopy coherent diagram $F: \mathscr{C} \rightarrow \mathscr{D}$, combining Formula (3.26) and Construction 3.8 gives a homotopy coherent diagram $G: \mathscr{C} \rightarrow \mathrm{Kom}$.

The $\mathbb{Z} / 2$-action on $M$ induces a $\mathbb{Z} / 2$-action on $\mathscr{D}$. Given sufficiently generic, $\tau$-equivariant homotopy coherent diagrams $F, F^{\prime}: \mathscr{E} \mathbb{Z} / 2 \rightarrow \overline{\mathcal{J}}$ used to define $\widetilde{C F}\left(L_{0}, L_{1}\right)$ and $\widetilde{C F}\left(L_{0}^{\prime}, L_{1}\right)$, by the same argument as in the proof of Lemma 3.13 we can extend $\{0\} \times F \cup\{1\} \times F^{\prime}$ to a sufficiently generic, $\tau$-equivariant homotopy coherent diagram $F^{\prime \prime}: \mathscr{I} \times \mathscr{E} \mathbb{Z} / 2 \rightarrow \mathscr{D}$. Applying Floer theory as above gives a homotopy coherent diagram $G^{\prime \prime}: \mathscr{I} \times \mathscr{E} \mathbb{Z} / 2 \rightarrow$ Kom extending $G \amalg G^{\prime}$. As noted after Formula (3.26), for each $x \in \operatorname{Ob}(\mathscr{E}), G^{\prime \prime}\left(f_{0,1} \times\right.$ $\left.\mathbb{I}_{x}\right)$ is a homotopy equivalence. Hence, $\operatorname{hocolim}\left(G^{\prime \prime}\right): \operatorname{hocolim} G \rightarrow \operatorname{hocolim} G^{\prime}$ is a quasiisomorphism over $\mathbb{F}_{2}[\mathbb{Z} / 2]$, as desired.

Finally, in the case that $L_{0} \cap L_{0}^{\prime} \cap L_{1}$ is nonempty, there may be constant triangles contained in $L_{0} \cap L_{0}^{\prime} \cap L_{1}$ which are not transversely cut out by the $\bar{\partial}$-operator for any almost complex structure. (For example, constant triangles may have negative Maslov index; see the proof of Lemma 6.10 for an example in which this occurs.) These are treated similarly to the discussion in Section 3.6. That is, extend the category $\mathscr{D}$ above to include small Hamiltonian perturbations of $L_{1}$ along the corresponding edge of the triangle, in the morphisms from $(0, J)$ to $\left(1, J^{\prime}\right)$. With this larger space of perturbations we are able to achieve transversality for all homotopy classes of triangles, and the proof proceeds as before.

3.6. Non-transverse intersections. Suppose next that $L_{0}$ and $L_{1}$ are $\mathbb{Z} / 2$-equivariant Lagrangians which do not intersect transversely. By incorporating the space of Hamiltonian perturbations of $L_{0}$, say, into the parameter space, along with the space of almost complex structures, we can still define an equivariant Floer complex. Fix an open neighborhood $U \ni$ $L_{0}$ which is preserved by the $\mathbb{Z} / 2$-action, let $C_{0}^{\infty}(U)$ denote the space of compactly-supported functions $H: U \rightarrow \mathbb{R}$, and let $\mathcal{H}$ denote the space of smooth maps $\mathbb{R} \rightarrow C_{0}^{\infty}(U)$ which are translation-invariant near $\pm \infty$. Let $\overline{\mathcal{H}}$ denote the category with objects elements of $C_{0}^{\infty}(U)$ and $\operatorname{Hom}\left(K_{0}, K_{1}\right)$ the space of sequences $\left(\widetilde{H}^{1}, \ldots, \widetilde{H}^{n}\right)$ of elements of $\mathcal{H}$ so that $\widetilde{H}^{1}$ agrees with $K_{0}$ at $-\infty, \widetilde{H}^{n}$ agrees with $K_{1}$ at $+\infty$, and for large enough $T$, the restriction of $\widetilde{H}_{i+1}$ to $(-\infty,-T)$ agrees with the restriction of $\widetilde{H}_{i}$ to $(T, \infty)$. Like $\overline{\mathcal{J}}$, the space $\operatorname{Hom}_{\overline{\mathcal{H}}}\left(K_{0}, K_{1}\right)$ has a natural topology, making $\overline{\mathcal{H}}$ into a topological category. The action of $\mathbb{Z} / 2$ on $M$ induces an action of $\mathbb{Z} / 2$ on $\overline{\mathcal{H}}$.

Given a generic $K \in C_{0}^{\infty}(U)$, the time-1 Hamiltonian flow $\phi_{K}$ of $K$ takes $L_{0}$ to a Lagrangian which is transverse to $L_{1}$ so, if we fix a generic almost complex structure $J$, we get a Floer complex $C F\left(L_{0}, L_{1} ; K ; J\right):=\left(C F\left(\phi_{K}\left(L_{0}\right), L_{1}\right), \partial_{J}\right)$. Similarly, given a generic path 
of Hamiltonians $H \in \operatorname{Hom}_{\overline{\mathcal{H}}}\left(K_{0}, K_{1}\right)$ and a path $J \in \operatorname{Hom}_{\overline{\mathcal{J}}}\left(J_{0}, J_{1}\right)$ there is a corresponding Floer continuation map $C F\left(L_{0}, L_{1} ; K_{0} ; J_{0}\right) \rightarrow C F\left(L_{0}, L_{1} ; K_{1} ; J_{1}\right)$. More generally, given a map $[0,1]^{m} \rightarrow \operatorname{Hom}_{\overline{\mathcal{H}}}\left(K_{0}, K_{1}\right) \times \operatorname{Hom}_{\overline{\mathcal{J}}}\left(J_{0}, J_{1}\right)$ there is a corresponding map

$$
C F\left(L_{0}, L_{1} ; K_{0} ; J_{0}\right) \rightarrow C F\left(L_{0}, L_{1} ; K_{1} ; J_{1}\right)
$$

of degree $m$, by counting holomorphic bigons for any point in the $[0,1]^{m}$-parameter family of (time-dependent) Hamiltonians and (time-dependent) almost complex structures. So, given a sufficiently generic homotopy coherent diagram $F: \mathscr{C} \rightarrow \overline{\mathcal{H}} \times \overline{\mathcal{J}}$ there is a corresponding homotopy coherent diagram $G: \mathscr{C} \rightarrow$ Kom.

Choose a sufficiently generic, $\mathbb{Z} / 2$-equivariant homotopy coherent diagram $F: \mathscr{E} \mathbb{Z} / 2 \rightarrow$ $\overline{\mathcal{H}} \times \overline{\mathcal{J}}$. (Since the space $C_{0}^{\infty}(U)$ is contractible, it is easy to see that such a diagram exists.) Let $G: \mathscr{E} \mathbb{Z} / 2 \rightarrow$ Kom be the corresponding $\mathbb{Z} / 2$-equivariant homotopy coherent diagram of complexes. We defined the freed Floer complex of $L_{0}$ and $L_{1}$ to be $\widetilde{C F}\left(L_{0}, L_{1}\right):=\operatorname{hocolim} G$, which is a module over $\mathbb{F}_{2}[\mathbb{Z} / 2]$, and the equivariant Floer cohomology to be $H F_{\mathbb{Z} / 2}\left(L_{0}, L_{1}\right):=$ $\operatorname{Ext}_{\mathbb{F}_{2}[\mathbb{Z} / 2]}\left(\right.$ hocolim $\left.G, \mathbb{F}_{2}\right)$. By the same argument as in Section 3.5, the freed Floer complex is well-defined up to quasi-isomorphism over $\mathbb{F}_{2}[\mathbb{Z} / 2]$, and is invariant, up to quasi-isomorphism over $\mathbb{F}_{2}[\mathbb{Z} / 2]$, under equivariant Hamiltonian isotopies of $L_{0}$ and $L_{1}$.

Lemma 3.27. If $L_{0}$ and $L_{1}$ intersect transversely then the new definition of the freed Floer complex agrees with the old one, up to quasi-isomorphism over $\mathbb{F}_{2}[\mathbb{Z} / 2]$.

Proof. We can simply take all of the Hamiltonians to be the constant function 0.

3.7. Other groups. One can imitate the construction above with $\mathbb{Z} / 2$ replaced by any finite group $K$ to obtain a $K$-equivariant Floer homology, though we will continue to work with coefficients in $\mathbb{F}_{2}$ to avoid discussing orientations of moduli spaces.

So, fix a finite group $K$ and an action of $K$ on a symplectic manifold $M$ by symplectomorphisms preserving a pair of Lagrangians $L_{0}$ and $L_{1}$. Assume that $\left(M, L_{0}, L_{1}\right)$ satisfy Hypothesis 3.2. Let $\mathscr{E}$ be any small category so that there is a unique morphism $a \rightarrow b$ for any pair of objects $a, b$ in $\mathscr{E}$. Further assume that we have a free right action by $K$ on the objects of $\mathscr{E}$ (which therefore extends uniquely to an $K$-action on $\mathscr{E}$ ). For concreteness, we can choose $\mathscr{E}$ to be the category $\mathscr{E} K$ which has one object $g$ for each element $g \in K$ and a single morphism $\underline{g} \rightarrow \underline{h}$ for each pair of objects $\underline{g}$ and $\underline{h}$. (So, every object in $\mathscr{E} K$ is initial and terminal and, in fact, $\mathscr{E} K$ depends only on the order of $K$.) There is a free right action of $K$ on $\mathscr{E} K$, by $g \cdot h=g h$.

By the same inductive argument as in Lemma 3.13, we can find a sufficiently generic, $K$-equivariant homotopy coherent diagram $F: \mathscr{E} \rightarrow \overline{\mathcal{J}}$. Construction 3.8 then gives a homotopy coherent functor $G: \mathscr{E} \rightarrow$ Kom. Define the freed Floer complex to be the complex $\widetilde{C F}\left(L_{0}, L_{1}\right):=\operatorname{hocolim} G$, which is a chain complex over $\mathbb{F}_{2}[K]$. (If more than one group is in play, we may denote $\widetilde{C F}\left(L_{0}, L_{1}\right)$ by $\widetilde{C F}^{K}\left(L_{0}, L_{1}\right)$ to emphasize which group we are considering.) The $K$-equivariant Floer complex is $C F_{K}\left(L_{0}, L_{1}\right)=\operatorname{Hom}_{\mathbb{F}_{2}[K]}\left(\right.$ hocolim $\left.G, \mathbb{F}_{2}\right)$ and the $K$-equivariant Floer homology $H F_{K}\left(L_{0}, L_{1}\right)$ is the homology of $C F_{K}\left(L_{0}, L_{1}\right)$. Since $\widetilde{C F}\left(L_{0}, L_{1}\right)$ is a bounded-below complex of free $\mathbb{F}_{2}[K]$-modules, we have $H F_{K}\left(L_{0}, L_{1}\right) \cong$ $\operatorname{Ext}_{\mathbb{F}_{2}[K]}\left(\widetilde{C F}\left(L_{0}, L_{1}\right), \mathbb{F}_{2}\right)$, so in particular there is an action (and, by homological perturbation theory, even an $A_{\infty}$-action) of $H^{*}(K)$ on $H F_{K}\left(L_{0}, L_{1}\right)$, and a spectral sequence $H^{*}\left(K, H F^{*}\left(L_{0}, L_{1}\right)\right) \Rightarrow H F_{\mathbb{Z} / 2}\left(L_{0}, L_{1}\right)$, both of which are invariants of the quasi-isomorphism type of $\widetilde{C F}\left(L_{0}, L_{1}\right)$ over $\mathbb{F}_{2}[K]$. 
Proposition 3.28. Up to quasi-isomorphism over $\mathbb{F}_{2}[K]$, the complex $\widetilde{C F}\left(L_{0}, L_{1}\right)$ is independent of the choice of $K$-equivariant sufficiently generic homotopy coherent diagram in $\mathcal{J}$ used to define it. If $L_{0}^{\prime}$ and $L_{1}^{\prime}$ are isotopic to $L_{0}$ and $L_{1}$ via compactly-supported, Hamiltonian isotopies through $K$-invariant Lagrangians then there is a quasi-isomorphism of $\mathbb{F}_{2}[K]$-modules $\widetilde{C F}\left(L_{0}, L_{1}\right) \simeq \widetilde{C F}\left(L_{0}^{\prime}, L_{1}^{\prime}\right)$.

The proof follows along the same lines as the proofs of Propositions 3.23 and 3.24, and is left to the reader. (As in Proposition 3.24 the complexes $\widetilde{C F}\left(L_{0}, L_{1}\right)$ and $\widetilde{C F}\left(L_{0}^{\prime}, L_{1}^{\prime}\right)$ are defined with respect to corresponding $K$-invariant collections of homotopy classes of paths, $\eta$ and $\eta^{\prime}$.)

We also have an analogue of Proposition 3.25:

Proposition 3.29. Suppose that $L_{0}, L_{0}^{\prime}$ and $L_{1}$ are $K$-invariant Lagrangians, which are pairwise transverse, and that $L_{0}$ is Hamiltonian isotopic to $L_{0}^{\prime}$. Suppose further that:

(1) The collection of homotopy classes $\eta^{\prime}$ induced by $\eta$ and the Hamiltonian isotopies is preserved by the $K$-action. (Of course, $\eta$ is also required to be preserved by the $K$-action, per Hypothesis 3.2.)

(2) There is a $K$-invariant, $\omega$-compatible almost complex structure $J$ on $M$ which achieves transversality for all moduli spaces of holomorphic bigons with boundary on $\left(L_{0}, L_{0}^{\prime}\right)$ of Maslov index $\leq 1$.

(3) The top class in $H F\left(L_{0}, L_{0}^{\prime}\right)$ (i.e., the isomorphism between $L_{0}$ and $L_{0}^{\prime}$ in the Fukaya category) is represented by a cycle $1 \in C F\left(L_{0}, L_{0}^{\prime}\right)$ which is fixed by the $K$-action.

Then there is a quasi-isomorphism $C F_{K}\left(L_{0}, L_{1}\right) \simeq C F_{K}\left(L_{0}^{\prime}, L_{1}\right)$ of chain complexes over $\mathbb{F}_{2}[K]$.

Again, we leave the generalization of the proof to the reader.

\section{Equivariant Floer homology Via EQUivariant COMPleX STRUCTURES}

In this section, we show that the equivariant Floer homology from Section 3 agrees with the equivariant Floer homology computed from an equivariant almost complex structure (as in [SS10, Sections 2b and 3]) essentially whenever the latter is defined.

4.1. The case of involutions. We start by briefly recalling the equivariant construction of equivariant Floer homology, in the case $H=\mathbb{Z} / 2$. Fix $M, L_{0}, L_{1}, \eta$, and an action of $\mathbb{Z} / 2=\{1, \tau\}$ as in Section 3, and in particular satisfying Hypothesis 3.2. Consider almost complex structures $J$ which are $\tau$-invariant, i.e., such that $d \tau \circ J \circ d \tau=J$. This is an infinite-codimension condition, and it may not be possible to find a $\tau$-invariant almost complex structure which achieves transversality for the $\bar{\partial}$ operator. We hypothesize away this difficulty:

Hypothesis 4.1. Assume that there is a one-parameter family of $\tau$-invariant almost complex structures $J=J(t), t \in[0,1]$ on $M$ compatible with $\omega$ and so that for any homotopy class of Whitney disks $\phi$ between $L_{0}$ and $L_{1}$ with Maslov index $\mu(\phi) \leq 1$, the moduli space of $J$-holomorphic disks $\mathcal{M}(\phi)$ in the homotopy class $\phi$ is transversely cut out.

Under this hypothesis, we can define the equivariant Floer complex as follows. The Floer complex $\left(C F\left(L_{0}, L_{1}\right), \partial_{J}\right)$, computed with respect to $J$, satisfies $\partial_{J}^{2}=0$ : even though the index 2 moduli spaces for $J$ may not be cut out transversally, there will be a (non-invariant) $J^{\prime}$ arbitrarily close to $J$ for which the index 2 moduli spaces are transversally cut out, 
so $\partial_{J^{\prime}}^{2}=0$, and for $J^{\prime}$ close enough to $J, \partial_{J}=\partial_{J^{\prime}}$. Further, since $J$ is $\tau$-invariant, the map $\tau_{\#}: C F\left(L_{0}, L_{1}\right) \rightarrow C F\left(L_{0}, L_{1}\right)$ induced by $\tau: L_{0} \cap L_{1} \rightarrow L_{0} \cap L_{1}$ is a chain map, so $C F\left(L_{0}, L_{1}\right)$ becomes a chain complex over $\mathbb{F}_{2}[\mathbb{Z} / 2]$. The equivariant Floer cohomology is the $\mathbb{Z} / 2$-equivariant cohomology of $C F\left(L_{0}, L_{1}\right)$, i.e., $\widetilde{\widehat{H F}}_{\mathbb{Z} / 2}\left(L_{0}, L_{1}\right)=\operatorname{Ext}_{\mathbb{F}_{2}[\mathbb{Z} / 2]}\left(C F\left(L_{0}, L_{1}\right), \mathbb{F}_{2}\right)$. Explicitly, this is the cohomology of the bicomplex

$$
\widetilde{\widetilde{C F}}_{\mathbb{Z} / 2}\left(L_{0}, L_{1}\right)=\left(0 \rightarrow\left(C F^{*}\left(L_{0}, L_{1}\right), d_{J}\right) \stackrel{\mathbb{I}+\tau^{\#}}{\longrightarrow}\left(C F^{*}\left(L_{0}, L_{1}\right), d_{J}\right) \stackrel{\mathbb{I}+\tau^{\#}}{\longrightarrow} \cdots\right)
$$

where $C F^{*}\left(L_{0}, L_{1}\right)$ is the Floer cochain complex, the dual (over $\mathbb{F}_{2}$ ) of $C F\left(L_{0}, L_{1}\right)$.

Proposition 4.3. Under Hypothesis 4.1, there is a quasi-isomorphism of $\mathbb{F}_{2}[\mathbb{Z} / 2]$-modules

$$
C F\left(L_{0}, L_{1}\right) \simeq \widetilde{C F}\left(L_{0}, L_{1}\right)
$$

between the Floer complex of $L_{0}$ and $L_{1}$ and the freed Floer complex (Definition 3.15).

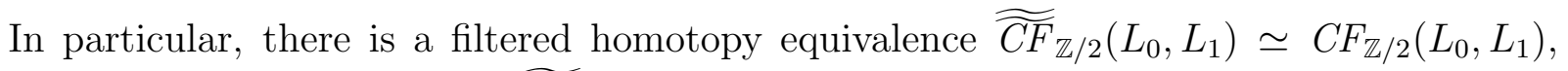
and the induced isomorphism $\widetilde{\widehat{H F}}_{\mathbb{Z} / 2}\left(L_{0}, L_{1}\right) \cong H F_{\mathbb{Z} / 2}\left(L_{0}, L_{1}\right)$ respects the $\mathbb{F}_{2}[\theta]$-module structure.

Proof. Since $J$ achieves transversality for the $\bar{\partial}$-operator, there is a small neighborhood $U$ of $J$ in the space of almost complex structures $\mathcal{J}$ so that for any cylindrical at infinity almost complex structure $\widetilde{J}: \mathbb{R} \rightarrow U$ contained in $U$ and any homotopy class $\phi$ of Whitney disks there is a homeomorphism $\mathcal{M}(\phi ; \widetilde{J}) \cong \mathcal{M}(\phi ; J)$. In particular, if $\mu(\phi)<0$ then $\mathcal{M}(\phi ; \widetilde{J})=\varnothing$ and if $\mu(\phi)=0$ then $\mathcal{M}(\phi ; \widetilde{J})=\varnothing$ unless $\phi$ is the constant disk from an intersection point $x$ to itself, in which case $\mathcal{M}(\phi ; \widetilde{J})$ consists of a single point.

In Lemma 3.13, we can choose the diagram $F$ so that each eventually cylindrical almost complex structure is contained in $U$. It follows that:

- $C F\left(L_{0}, L_{1} ; F(a)\right) \cong C F\left(L_{0}, L_{1} ; F(b)\right) \cong C F\left(L_{0}, L_{1} ; J\right)$, as chain complexes, via the identity map $L_{0} \cap L_{1} \rightarrow L_{0} \cap L_{1}$.

- $G_{f_{1}, \ldots, f_{n}}=0$ for any sequence of composable morphisms $\left\{f_{i}\right\} \subset\{\alpha, \beta\}$ of length $n>1$.

- $G_{\alpha}$ and $G_{\beta}$ are induced by the identity maps $L_{0} \cap L_{1} \rightarrow L_{0} \cap L_{1}$.

It follows from Equations (3.17) and (3.18) that

$$
\begin{aligned}
& \partial\left(\alpha_{n} \otimes x\right)=\alpha_{n} \otimes(\partial x)+\alpha_{n-1} \otimes x+\beta_{n-1} \otimes x \\
& \partial\left(\beta_{n} \otimes x\right)=\beta_{n} \otimes(\partial x)+\beta_{n-1} \otimes x+\alpha_{n-1} \otimes x
\end{aligned}
$$

We will take the quotient of $\widetilde{C F}\left(L_{0}, L_{1}\right)$ by an acyclic complex preserved by $\tau$, and show that the result is exactly $C F\left(L_{0}, L_{1}\right)$ as an $\mathbb{F}_{2}[\mathbb{Z} / 2]$-module. Consider the following change of basis: replace $\beta_{n} \otimes x$ with

$$
\xi_{n}(x)=\beta_{n} \otimes x+\alpha_{n} \otimes x+\alpha_{n+1} \otimes \partial(x) .
$$

Then our differential becomes

$$
\begin{aligned}
& \partial\left(\xi_{n}(x)\right)=0 \\
& \partial\left(\alpha_{n} \otimes x\right)=\xi_{n-1}(x) \quad \text { for } n \geq 1 .
\end{aligned}
$$

Let $C$ be the subcomplex of $\widetilde{C F}\left(L_{0}, L_{1}\right)$ spanned by $\left\{a_{n} \otimes x, \xi_{m}(x): x \in L_{0} \cap L_{1}, n \geq 1, m \geq\right.$ $0\}$, or equivalently spanned by $\left\{\alpha_{n} \otimes x, \beta_{n} \otimes x, \alpha_{0} \otimes x+\beta_{0} \otimes x: x \in L_{0} \cap L_{1}, n \geq 1\right\}$. From the 
second description it is clear $C$ is preserved by the $\mathbb{Z} / 2$-action. Furthermore, the quotient of $C F\left(L_{0}, L_{1}\right)$ by $C$ is spanned by elements $\left\{\alpha_{0} \otimes x: x \in L_{0} \cap L_{1}\right\}$ with $\partial\left(\alpha_{0} \otimes x\right)=\alpha_{0} \otimes(\partial x)$ and $\mathbb{Z} / 2$ action

$$
\tau_{\#}\left(\alpha_{0} \otimes x\right)=\beta_{0} \otimes \tau_{\#} x \equiv \alpha_{0} \otimes \tau_{\#} x .
$$

This is exactly $C F\left(L_{0}, L_{1}\right)$.

It is not entirely clear how restrictive Hypothesis 4.1 is, but it is satisfied in at least two important cases:

(ET-1) Let $N M^{f i x}, N L_{0}^{f i x}$ and $N L_{1}^{f i x}$ denote the normal bundles to the $\tau$-fixed parts $M^{f i x}$, $L_{0}^{f i x}$, and $L_{1}^{f i x}$ in $M, L_{0}$, and $L_{1}$. Suppose that there is an equivariant trivialization $N M^{f i x} \cong M^{f i x} \times \mathbb{C}^{k}$, where $\tau$ acts on $\mathbb{C}^{k}$ by $\tau(\vec{z})=-\vec{z}$, which sends $N L_{0}^{f i x}$ and $N L_{1}^{f i x}$ to $L_{0}^{f i x} \times \mathbb{R}^{k}$ and $L_{1}^{f i x} \times i \mathbb{R}^{k}$, respectively. Then Seidel-Smith observe that there is a one-parameter family of $\tau$-invariant almost complex structures $J$ on $M$ achieving transversality for all moduli spaces of $J$-holomorphic disks [SS10, Lemma 19].

(ET-2) Let $i:\left(M^{f i x}, L_{0}^{f i x}, L_{1}^{f i x}\right) \rightarrow\left(M, L_{0}, L_{1}\right)$ denote inclusion. Suppose that for any homotopy class of Whitney disks $\phi:\left(\mathbb{D}^{2}, \partial \mathbb{D}^{2}\right) \rightarrow\left(M^{f i x}, L_{0} \cup L_{1}\right), \mu\left(i_{*}(\phi)\right)=2 \mu(\phi)$ (where $i_{*}(\phi)$ denotes the homotopy class of disks in $\left(M, L_{0} \cup L_{1}\right)$ corresponding to $\left.\phi\right)$. Then Hypothesis 4.1 is satisfied. To see this, choose a one-parameter family of almost complex structures $J_{f i x}$ on $M^{f i x}$ which achieves transversality for holomorphic disks in $M^{f i x}$. (Note that the Hypothesis 3.2 for $M$ and the condition $\mu\left(i_{*}(\phi)\right)=2 \mu(\phi)$ imply Hypothesis 3.2 for $M^{f i x}$.) As discussed in [KS02, Section 5c], a generic choice of $\mathbb{Z} / 2$-invariant extension of $J_{f i x}$ to an almost complex structure $J$ on $M$ will achieve transversality for all holomorphic disks not contained entirely in $M^{f i x}$. But since $\mu\left(i_{*}(\phi)\right)=2 \mu(\phi)$ and $J_{f i x}$ achieves transversality in $M^{f i x}$, there are no nontrivial holomorphic curves in $M^{f i x}$ with index $\leq 1$.

Seidel-Smith show that if $\left(M, L_{0}, L_{1}, \tau\right)$ satisfy a weaker (but still restrictive) condition, stable normal triviality, then after an isotopy one can arrange for Condition (ET-1) to be satisfied. As was already observed in [LL08] and further exploited in [Hen12,Hen15], Condition (ET-2) arises in $\mathbb{Z} / 2$-covers of Heegaard diagrams. Both points will be used in Section 5.

Proof of Theorem 1.1. Seidel-Smith's equivariant Floer complex is $\widetilde{\widetilde{C F}}_{\mathbb{Z} / 2}\left(L_{0}, L_{1}\right)$, which is well-defined in their setting because of Point (ET-1). It follows from Proposition 4.3 that their filtered complex agrees with $C F_{\mathbb{Z} / 2}\left(L_{0}, L_{1}\right)$, and by Propositions 3.23 and 3.24 that the filtered homotopy type of $C F_{\mathbb{Z} / 2}\left(L_{0}, L_{1}\right)$ is independent of auxiliary choices and invariant under equivariant Hamiltonian isotopies.

4.2. General finite groups. Finally, we turn briefly to general finite groups $K$. With notation as in Section 3.7, we have the following generalization of Hypothesis 4.1:

Hypothesis 4.4. Assume that there is a one-parameter family of K-invariant almost complex structures $J=J(t), t \in[0,1]$ on $M$ compatible with $\omega$ and so that for any homotopy class of Whitney disks $\phi$ between $L_{0}$ and $L_{1}$ with Maslov index $\mu(\phi) \leq 1$, the moduli space of J-holomorphic disks $\mathcal{M}(\phi)$ in the homotopy class $\phi$ is transversely cut out.

In this case, $C F\left(L_{0}, L_{1}\right)$ becomes a module over $\mathbb{F}_{2}[K]$. To define the equivariant Floer complex, choose a projective resolution $R_{*}$ of $C F\left(L_{0}, L_{1}\right)$ over $\mathbb{F}_{2}[K]$, and define $\widetilde{\widetilde{C F}}_{\mathbb{Z} / 2}\left(L_{0}, L_{1}\right)=$ $\operatorname{Hom}_{\mathbb{F}_{2}[K]}\left(R_{*}, \mathbb{F}_{2}\right)$ and $\widetilde{\overparen{H F}}_{\mathbb{Z} / 2}\left(L_{0}, L_{1}\right)=H_{*}\left(\widetilde{\widetilde{C F}}_{\mathbb{Z} / 2}\left(L_{0}, L_{1}\right)\right)=\operatorname{Ext}_{\mathbb{F}_{2}[\mathbb{Z} / 2]}\left(C F\left(L_{0}, L_{1}\right), \mathbb{F}_{2}\right)$. (To 
give an explicit definition of $\widetilde{C F}_{\mathbb{Z} / 2}\left(L_{0}, L_{1}\right)$ we can take $R_{*}$ to be the bar resolution.) The analogue of Proposition 4.3 is:

Proposition 4.5. Under Hypothesis 4.4, there is a quasi-isomorphism of $\mathbb{F}_{2}[K]$-modules

$$
C F\left(L_{0}, L_{1}\right) \simeq \widetilde{C F}\left(L_{0}, L_{1}\right)
$$

between the Floer complex of $L_{0}$ and $L_{1}$ and the freed Floer complex (Definition 3.15).

Proof. Fix a one-parameter family of almost complex structures as in Hypothesis 4.4 and induce a homotopy coherent diagram $F: \mathscr{E} K \rightarrow \overline{\mathcal{J}}$ as in the proof of Proposition 4.3. If $G: \mathscr{E} K \rightarrow$ Kom is the homotopy coherent diagram induced by $F$ and Floer theory then recall that

$$
\operatorname{hocolim} G=\bigoplus_{n \geq 0} \bigoplus_{g_{0}, \ldots, g_{n} \in K} I_{*}^{\otimes n} \otimes G\left(g_{0}\right) / \sim
$$

There is a map hocolim $G \rightarrow C F\left(L_{0}, L_{1}\right)$ which is:

- The identity map $I_{*}^{\otimes 0} \otimes G\left(g_{0}\right)=C F\left(L_{0}, L_{1}\right) \rightarrow C F\left(L_{0}, L_{1}\right)$ for $n=0$.

- Vanishes on $\alpha \otimes G\left(g_{0}\right)$ if $\alpha \in I_{*}^{\otimes n}$ has grading $>0$.

It is straightforward to verify that this map is $K$-equivariant, and it follows from Lemma 3.14 (or rather, its extension to arbitrary groups $K$ ) that this map is a quasi-isomorphism. The result follows.

We will not formulate the analogue of Condition (ET-1), but the analogue of Condition $(\mathrm{ET}-2)$ is:

$\left(\mathrm{ET}-2^{\prime}\right)$ Let $i:\left(M^{f i x}, L_{0}^{f i x}, L_{1}^{f i x}\right) \rightarrow\left(M, L_{0}, L_{1}\right)$ denote inclusion. Suppose that for any homotopy class of Whitney disks $\phi:\left(\mathbb{D}^{2}, \partial \mathbb{D}^{2}\right) \rightarrow\left(M^{f i x}, L_{0} \cup L_{1}\right), \mu\left(i_{*}(\phi)\right)=c \mu(\phi)$ for some $c \neq 1$ a divisor of $|K|$.

Condition (ET-2') implies Hypothesis 4.4, since it rules out index 1 holomorphic curves fixed by nontrivial elements of $K$.

\section{SpeCtRAL SEQUENCES FOR PERIODIC KNOTS AND BRANCHED DOUBLE COVERS}

5.1. First invariance statements. As a warm-up, we prove Corollaries 1.10 and 1.11, that Hendricks's spectral sequences are knot invariants. We start by recalling how the spectral sequences are constructed. For the spectral sequence (1.6), start with a multi-pointed, genus-0 Heegaard diagram $\mathcal{H}=\left(S^{2}, \boldsymbol{\alpha}, \boldsymbol{\beta}, \mathbf{z}, \mathbf{w}\right)$ for $\left(S^{3}, K\right)$. Let $\widetilde{\mathcal{H}}=(\widetilde{\Sigma}, \widetilde{\boldsymbol{\alpha}}, \widetilde{\boldsymbol{\beta}}, \widetilde{\mathbf{z}}, \widetilde{\mathbf{w}})$ be the double cover of $\mathcal{H}$ branched along $\mathbf{z} \cup \mathbf{w}$ so that $\widetilde{\mathcal{H}}$ represents $(\Sigma(K), K)$. Let $n=|\boldsymbol{\alpha}|$ denote the number of $\alpha$-circles, so $|\boldsymbol{\beta}|=n$ and $|\mathbf{z}|=|\mathbf{w}|=|\widetilde{\mathbf{z}}|=|\widetilde{\mathbf{w}}|=n+1$, and $|\widetilde{\boldsymbol{\alpha}}|=|\widetilde{\boldsymbol{\beta}}|=2 n$. Consider the symmetric products $\operatorname{Sym}^{n}\left(S^{2} \backslash(\mathbf{z} \cup \mathbf{w})\right)$ and $\operatorname{Sym}^{2 n}(\widetilde{\Sigma} \backslash(\widetilde{\mathbf{z}} \cup \widetilde{\mathbf{w}}))$, and the submanifolds $T_{\alpha}=\prod \boldsymbol{\alpha}, T_{\beta}=\prod \boldsymbol{\beta} \subset \operatorname{Sym}^{n}\left(S^{2} \backslash(\mathbf{z} \cup \mathbf{w})\right)$ and $\widetilde{T}_{\alpha}=\prod \widetilde{\boldsymbol{\alpha}}, \widetilde{T}_{\beta}=$ $\prod \widetilde{\boldsymbol{\beta}} \subset \operatorname{Sym}^{2 n}(\widetilde{\Sigma} \backslash(\widetilde{\mathbf{z}} \cup \widetilde{\mathbf{w}}))$. There is an involution $\tau: \widetilde{\mathcal{H}} \rightarrow \widetilde{\mathcal{H}}$, which induces a symplectic involution $\tau: \operatorname{Sym}^{2 n}(\widetilde{\Sigma}) \rightarrow \operatorname{Sym}^{2 n}(\widetilde{\Sigma})$ (with respect to an appropriate symplectic form), which preserves $\widetilde{T}_{\alpha}$ and $\widetilde{T}_{\beta}$. The fixed set of $\tau$ is identified with $\left(\operatorname{Sym}^{n}\left(S^{2}\right), T_{\alpha}, T_{\beta}\right)$. Using the fact that we are considering a genus-0 Heegaard diagram for $K$, one can show that Seidel-Smith's stable normal triviality conditions are satisfied on the symmetric products with the basepoints deleted. The spectral sequence (1.6) is then obtained by performing an equivariant Hamiltonian isotopy to get a new pair of Lagrangians $\left(\widetilde{T}_{\alpha}^{\prime}, \widetilde{T}_{\beta}^{\prime}\right)$ and then considering the equivariant Floer complex $\widetilde{C F}_{\mathbb{Z} / 2}\left(\widetilde{T}_{\alpha}^{\prime}, \widetilde{T}_{\beta}^{\prime}\right)$ from Section 4. 
The spectral sequence (1.8) is obtained similarly, except that one starts with a Heegaard diagram for the link $K \cup A$, so that exactly one $z$ and one $w$ basepoint correspond to $A$, and takes the branched double cover only over the two basepoints corresponding to $A$. The construction of the spectral sequence (1.9) is similar to the construction of (1.8) except that one does not delete the basepoint $\widetilde{z}$ corresponding to the axis $\widetilde{A}$.

Any two multi-pointed Heegaard diagrams for $K$ with a fixed number of $w$-basepoints, say $n-1$, can be connected by a sequence of isotopies of the $\alpha$ - and $\beta$-circles, handleslides among the circles, and index 1-2 stabilizations and destabilizations (connected sums with the standard genus 1 Heegaard diagram for $S^{3}$ ). Almost all of these moves are easily seen to induce Hamiltonian isotopies of the Lagrangians. There is one case that is of particular note, which we explain here.

Lemma 5.1. Let $\mathcal{H}=(\Sigma, \boldsymbol{\alpha}, \boldsymbol{\beta}, \mathbf{z}, \mathbf{w})$ be a multi-pointed Heegaard diagram for $\left(S^{3}, K\right)$ where $\Sigma$ has genus $g$ and $|\mathbf{w}|=n+1$. If $\mathcal{H}^{\prime}=\left(\Sigma, \boldsymbol{\alpha}^{\prime}, \boldsymbol{\beta}, \mathbf{z}, \mathbf{w}\right)$ is obtained from $\mathcal{H}$ via a handleslide, then for an appropriate choice of symplectic form on $\operatorname{Sym}^{g+n}(\Sigma \backslash \mathbf{w})$, the tori $T_{\alpha}$ and $T_{\alpha^{\prime}}$ are Hamiltonian isotopic in $\operatorname{Sym}^{g+n}(\Sigma \backslash \mathbf{w})$. Furthermore, the isotopy can be taken to occur in the complement of the divisor $\mathbf{z} \times \operatorname{Sym}^{g+n-1}(\Sigma \backslash \mathbf{w})$. An analogous result is true for $a$ multi-pointed Heegaard diagram for a three-manifold.

Proof. For a Heegaard diagram with a single $w$ basepoint, this was shown by Perutz [Per08, Theorem 1.2]. In order to deduce the result for a Heegaard diagram with $|\mathbf{w}|=n$, we attach $n$ one-handles to $\Sigma$ with feet near the basepoints $w_{i}$ and $w_{i+1}$, creating a genus $g+n$ surface $\Sigma^{\prime}$ with one basepoint. By Perutz's result [Per08, Theorem 1.2], there is a symplectic form $\omega$ on $\operatorname{Sym}^{g+n}\left(\Sigma^{\prime}\right)$ with respect to which $T_{\alpha}$ and $T_{\alpha^{\prime}}$ are Hamiltonian isotopic. Further, if we let $\boldsymbol{\alpha}=\left(\alpha_{1}, \ldots, \alpha_{g+n}\right)$ and $\boldsymbol{\alpha}^{\prime}=\left(\alpha_{1}^{\prime}, \alpha_{2}, \ldots, \alpha_{g+n}\right)$, where $\alpha_{1}^{\prime}$ is obtained from $\alpha_{1}$ by handlesliding over $\alpha_{2}$, then this isotopy takes place in $\alpha_{3} \times \cdots \times \alpha_{g+n} \times \operatorname{Sym}^{2}(U)$, where $U$ is a small pair-of-pants neighborhood of the handleslide region. In particular, we can choose $U$ to lie in $\Sigma$ and contain no basepoints (either $z$ or $w$ ), so this product is contained in $\operatorname{Sym}^{g+n}(\Sigma \backslash \mathbf{w})$. Therefore, restricting $\omega$ to the submanifold $\operatorname{Sym}^{g+n}(\Sigma \backslash \mathbf{w})$ gives the desired result. The argument for three-manifolds is identical, except that there is no need to avoid $z$ basepoints.

Proof of Corollary 1.10. By Proposition 4.3, the spectral sequence is the same as the spectral sequence induced by $C F_{\mathbb{Z} / 2}\left(\widetilde{T}_{\alpha}, \widetilde{T}_{\beta}\right)$, the equivariant complex from non-invariant complex structures (Section 3). By Propositions 3.23 and 3.24, this spectral sequence is independent of the choices in its construction and is invariant under equivariant Hamiltonian isotopies of the Lagrangians.

As observed by Ozsváth-Szabó [OSz04b, Section 7.3], isotopies of the $\alpha$ - and $\beta$-circles can be realized through deformations of the almost complex structures and Hamiltonian isotopies of the $\alpha$ - and $\beta$-tori. By Lemma 5.1, handleslides can also be realized as Hamiltonian deformations of the $\alpha$ - and $\beta$-tori. The Hamiltonian isotopies of the Lagrangians $T_{\alpha}$ and $T_{\beta}$ in $\operatorname{Sym}^{n}(\Sigma)$ lift to equivariant Hamiltonian isotopies of the Lagrangians $\widetilde{T}_{\alpha}$ and $\widetilde{T}_{\beta}$ in $\operatorname{Sym}^{2 n}(\widetilde{\Sigma})$.

Finally, we may assume that index 1-2 stabilizations occur near the basepoints $z \in \Sigma$ and so, if we choose our complex structures to be split near the basepoints, these stabilizations have no effect at all on the equivariant Floer complex (cf. [OSz04b, Proof of Theorem 10.1]). 
Of course, we have actually proved a little more: the quasi-isomorphism type of the complex $\widehat{C F K}(\Sigma, \boldsymbol{\alpha}, \boldsymbol{\beta}, \mathbf{z}, \mathbf{w})$, over $\mathbb{F}_{2}[\mathbb{Z} / 2]$, is an invariant of $(K, n)$.

Proof of Corollary 1.11. This is similar to the proof of Corollary 1.10, and is left to the reader.

5.2. Computing spectral sequences from equivariant diagrams. Now that we know the spectral sequences are knot invariants, it would be nice to be able to compute them. Corollary 1.12 says that in many cases we can compute the spectral sequences (1.6) and (1.8) directly from a diagram; Corollary 1.13 says that we can always find diagrams for which the spectral sequences (1.6), (1.8), and (1.9) are algorithmically computable.

While proving Corollary 1.13 for the spectral sequences (1.6) and (1.8), we will be able to use any genus- 0 nice Heegaard diagram for $K$ or $K \cup A$, as appropriate. However, to show the same result for the spectral sequence (1.9), we will need to restrict to a class of specially adapted nice diagrams for $K \cup A$, here called desirable diagrams. Beginning with a two-periodic knot diagram $\widetilde{D}$ for $\widetilde{K}$, we construct a planar grid diagram similar to Beliakova's diagrams [Bel10] as follows. We begin by deforming $\widetilde{D}$ equivariantly to a diagram $\widetilde{D}^{\prime}$ made up of horizontal and vertical line segments, such that all overcrossing strands in $\widetilde{D}^{\prime}$ are vertical and all undercrossing strands are horizontal. (This differs from the standard algorithm of placing a knot on a grid diagram only in the equivariance requirement.) Because of the requirement that we do this equivariantly, there will be even numbers of vertical and horizontal strands, say $2 n$ each. We circle each horizontal strand with an $\alpha$ curve and each vertical strand with a $\beta$ curve such that each $\alpha$ curve intersects each $\beta$ curve in four points, and we place a $w_{i}$ at the beginning of each vertical strand and a $z_{i}$ at the end. We place a single $w_{i}$ outside the grid and a single $z_{i}$ in the center of the grid; these are the basepoints on the axis $\widetilde{A}$. The quotient of this diagram by the $\mathbb{Z} / 2$ action is a Heegaard diagram for $K \cup A$ with $n \alpha$-circles, such that each $\alpha$ curve intersects each $\beta$ curve eight times. The $z$ basepoint belonging to the axis, in the center of the diagram, is contained in a bigon. A diagram for the trefoil as a two-periodic knot with quotient a desirable diagram for the unknot are shown in Figure 1.

Proof of Corollary 1.12. We explain the argument in the case of branched double covers; the case of periodic knots is similar. Given a 1-parameter family of complex structures $j$ on $S^{2}$ there is an induced 1-parameter family of almost complex structures $\operatorname{Sym}^{n}(j)$ on $\operatorname{Sym}^{n}\left(S^{2}\right)$. Given a generic choice of $j$ the complex structures $\operatorname{Sym}^{n}(j)$ achieve transversality for all moduli spaces of holomorphic disks in $\left(\operatorname{Sym}^{n}\left(S^{2} \backslash(\mathbf{z} \cup \mathbf{w})\right), T_{\alpha} \cup T_{\beta}\right)$ [Lip06, Proposition 13.5]. Further, the pullback $\widetilde{\jmath}$ of $j$ to $\widetilde{\Sigma}$ induces a one-parameter family of almost complex structures $\operatorname{Sym}^{2 n}(\widetilde{\jmath})$ on $\operatorname{Sym}^{2 n}(\widetilde{\Sigma} \backslash(\widetilde{\mathbf{z}} \cup \widetilde{\mathbf{w}}))$. The same argument which yields [Lip06, Proposition 13.5] implies that, for a generic choice of $j, \operatorname{Sym}^{2 n}(\widetilde{\jmath})$ achieves transversality for all homotopy classes of Whitney disks $\phi$ in $\operatorname{Sym}^{2 n}(\widetilde{\Sigma} \backslash(\widetilde{\mathbf{z}} \cup \widetilde{\mathbf{w}}))$ which are not $\tau$-invariant, i.e., for which $\phi \neq \tau_{*}(\phi)$.

Any $\tau$-invariant homotopy class $\phi$ has Maslov index $\mu(\phi)$ even. Thus, as discussed in Point (ET-2) of Section 4, Hypothesis 4.1 is satisfied. So, by Proposition 4.3, the equivariant complex $\widetilde{C F}_{\mathbb{Z} / 2}\left(\widetilde{T}_{\alpha}, \widetilde{T}_{\beta}\right)$ computed with respect to $\operatorname{Sym}^{2 n}(\widetilde{\jmath})$ agrees with the equivariant complex $C F_{\mathbb{Z} / 2}\left(\widetilde{T}_{\alpha}, \widetilde{T}_{\beta}\right)$ computed using non-equivariant almost complex structures. As in 

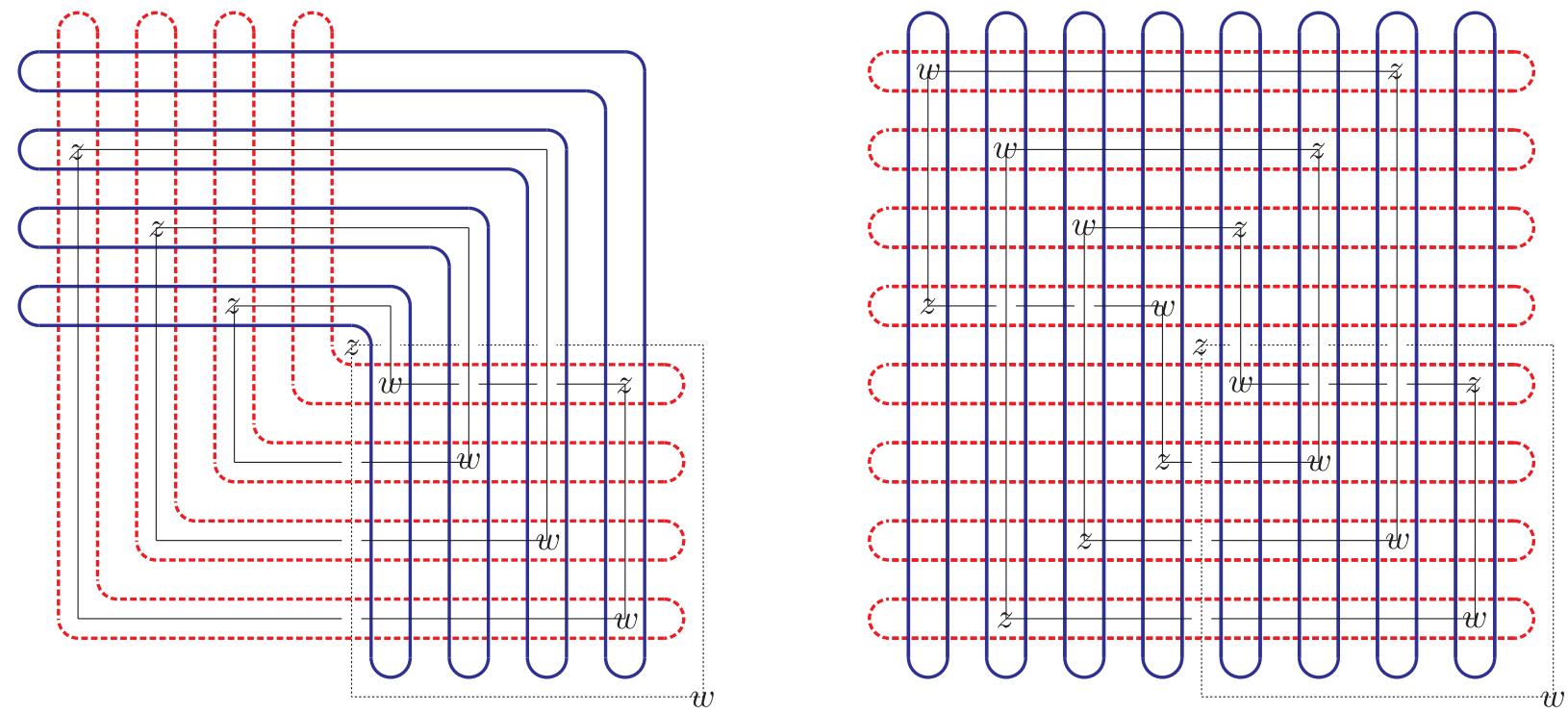

Figure 1. Desirable diagrams for links. Left: a desirable diagram for $K \cup A$, where $K$ is the unknot. Right: the corresponding 2-periodic diagram for $\widetilde{K} \cup \widetilde{A}$, where $\widetilde{K}$ is the trefoil. $\alpha$ 's are red and dashed, $\beta$ 's are blue and solid, axes thin, black and dashed and the knots thin-black, too.

the proof of Corollary 1.10, $C F_{\mathbb{Z} / 2}\left(\widetilde{T}_{\alpha}, \widetilde{T}_{\beta}\right)$ is isomorphic, in turn, to the equivariant Floer homology as defined by Seidel-Smith.

Proof of Corollary 1.13. For the spectral sequences (1.6) and(1.8), this is immediate from Corollary 1.12, the fact that the double cover of a nice Heegaard diagram branched over the basepoints is nice (obvious), and the fact that curve counts in nice diagrams are combinatorial [SW10]. For Corollary (1.9), curves are allowed to pass over the $z$ basepoint on the axis, and therefore we need the additional fact that in our desirable diagram for $K \cup A$, the $z$ basepoint for $A$ is contained in a bigon, which ensures that in the diagram for $\widetilde{K} \cup \widetilde{A}$, the $z$ basepoint for $\widetilde{A}$ is contained in a rectangle.

5.3. Equivariantly destabilizing basepoints and invariance of the spectral sequences. In this section, we prove that one can remove the extra copies of $V$ from Hendricks's spectral sequences (1.6), (1.8), and (1.9). We start by reviewing why extra basepoints give copies of $V$ in the first place [OSz08, MOS09].

Any two diagrams for a link $L$ with the same number of basepoints per component of $L$ are related by a sequence of isotopies, handleslides, and index 1-2 stabilizations/destabilizations, none of which affect the Floer homology $\widehat{H F L}(Y, L)$. Thus, it suffices to consider the effect of replacing a single basepoint $z$ with the $z-w-z$ triple on the left of Figure 2 . Let $\mathcal{H}$ denote the original diagram and $\mathcal{H}^{\prime}$ the new diagram, so $\mathcal{H}^{\prime}=\mathcal{H} \#\left(S^{2}, \alpha_{0}, \beta_{0},\left\{z_{0}, z_{0}^{\prime}\right\},\left\{w_{0}\right\}\right)$, and the connected sum occurs near one of the basepoints $z$ of $\mathcal{H}$ and a point $p$ in the new $S^{2}$. Let $\left\{x_{0}, y_{0}\right\}=\alpha_{0} \cap \beta_{0}$, labeled so that there is a bigon in $S^{2} \backslash\left\{z_{0}, z_{0}^{\prime}, w_{0}\right\}$ from $x_{0}$ to $y_{0}$.

There is an obvious correspondence between generators of $\widehat{C F K}\left(\mathcal{H}^{\prime}\right)$ and $\widehat{C F K}(\mathcal{H}) \otimes$ $\mathbb{F}_{2}\left\langle x_{0}, y_{0}\right\rangle$. We claim that for appropriate choices of complex structure, this correspondence is a chain map. Note first that for any homotopy class $\phi \in \pi_{2}(\mathbf{x}, \mathbf{y})$ of Whitney disks in $\mathcal{H}$ 

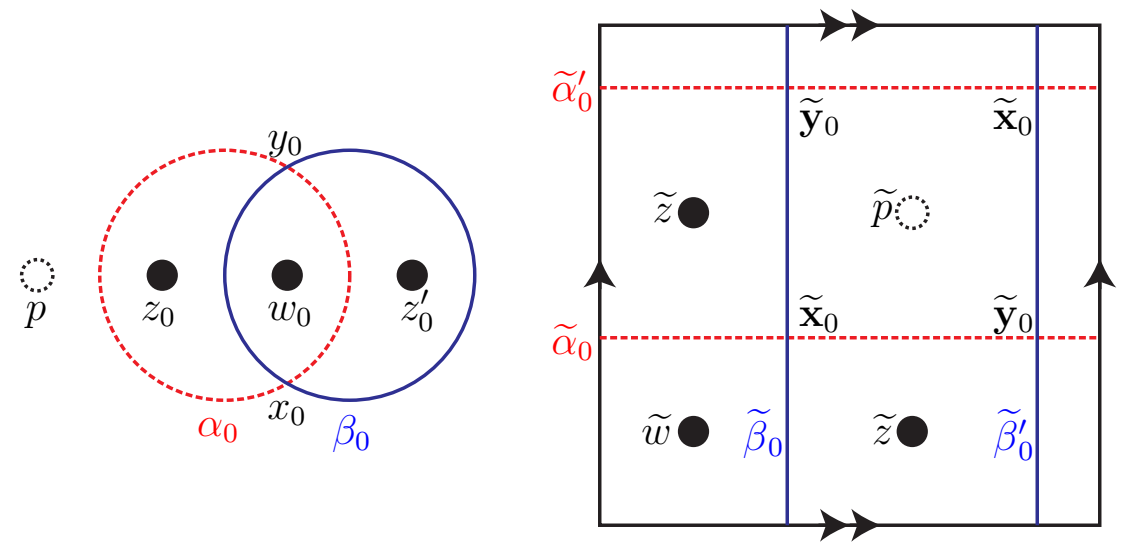

FiguRE 2. Stabilizations of type 0-1-2-3. Left: a stabilization replacing a $z$ basepoint by a $(z, w, z)$ triple. Right: the branched double cover of this stabilization.

with multiplicity 0 at the basepoints there are corresponding homotopy classes of Whitney disks $\phi_{x} \in \pi_{2}\left(\mathbf{x} \cup\left\{x_{0}\right\}, \mathbf{y} \cup\left\{x_{0}\right\}\right)$ and $\phi_{y} \in \pi_{2}\left(\mathbf{x} \cup\left\{y_{0}\right\}, \mathbf{y} \cup\left\{y_{0}\right\}\right)$ in $\mathcal{H}^{\prime}$. Assuming we work with complex structures of the form $\operatorname{Sym}^{n}(j)$ and $\operatorname{Sym}^{n^{\prime}}\left(j^{\prime}\right)$ so that $j^{\prime}$ agrees with $j$ on $\Sigma$ away from the connected sum region, there are obvious bijections $\mathcal{M}(\phi) \cong \mathcal{M}\left(\phi_{x}\right) \cong \mathcal{M}\left(\phi_{y}\right)$.

It remains to show there are no other holomorphic disks for $\mathcal{H}^{\prime}$. The only other homotopy classes of Whitney disks have multiplicity 1 in the connected sum region, and lie in $\pi_{2}(\mathbf{x} \cup$ $\left.\left\{x_{0}\right\}, \mathbf{y} \cup\left\{y_{0}\right\}\right)$ for some $\mathbf{x}, \mathbf{y} \in \widehat{C F K}(\mathcal{H})$. Fix such a homotopy class $\phi^{\prime}$, and consider the result of stretching the neck in the connected sum region. The moduli space $\mathcal{M}\left(\phi^{\prime}\right)$ in $\mathcal{H}^{\prime}$ decomposes as a fibered product

$$
\mathcal{M}\left(\phi^{\prime}\right) \cong \mathcal{M}(\phi) \times_{\mathbb{D}^{2}} \mathcal{M}\left(\phi_{0}\right)
$$

where $\phi$ (respectively $\phi_{0}$ ) is the domain in $\mathcal{H}$ (respectively $S^{2}$ ) induced by $\phi^{\prime}$. The domain $\phi$ (respectively $\phi_{0}$ ) has multiplicity 1 at $z$ (respectively $p$ ). The map $\mathcal{M}(\phi) \rightarrow \mathbb{D}^{2}$ (respectively $\left.\mathcal{M}\left(\phi_{0}\right) \rightarrow \mathbb{D}^{2}\right)$ involved in the fiber product sends $u$ to $u^{-1}\left(\{z\} \times \operatorname{Sym}^{n-1}(\Sigma)\right) \in \mathbb{D}^{2}$ (respectively $u_{0}$ to $u_{0}^{-1}(p)$ ).

Now, leave $z$ alone and make the connected sum point $p$ approach $\alpha_{0}$. As $p$ approaches $\alpha_{0}$, the point $u_{0}^{-1}(p) \in \mathbb{D}^{2}$ approaches the boundary of $\mathbb{D}^{2}$. Hence, if the fibered product is nonempty, $u^{-1}(z)$ also approaches $\partial \mathbb{D}^{2}$. But $u\left(\partial \mathbb{D}^{2}\right) \subset T_{\alpha} \cup T_{\beta}$ and $z \notin T_{\alpha} \cup T_{\beta}$, a contradiction. It follows that the fibered product is empty, and so, for large neck length and $p$ close to $\alpha_{0}$, $\mathcal{M}\left(\phi^{\prime}\right)$ is empty, as well.

With this understanding, we are ready to prove Theorems 1.14 and 1.16, and Corollaries 1.15 and 1.19 .

Proof of Theorem 1.14. By induction, it suffices to consider a single 0-1-2-3 destabilization (i.e., removing a single pair of basepoints). By Corollary 1.12, we can use a generic oneparameter family of almost complex structures $j$ on $S^{2}$ and the induced $\mathbb{Z} / 2$-invariant almost complex structure $\tilde{\jmath}$ on $\Sigma$ to compute the spectral sequence.

The double cover of $\left(S^{2}, \alpha_{0}, \beta_{0},\left\{z_{0}, z_{0}^{\prime}\right\},\left\{w_{0}\right\}\right)$, branched along $\left\{z_{0}, z_{0}^{\prime}, w_{0}, p\right\}$, is shown on the right of Figure 2. There is an obvious correspondence between generators of $\widehat{C F K}\left(\widetilde{\mathcal{H}}^{\prime}\right)$ and generators of $\widehat{C F K}(\widetilde{\mathcal{H}}) \otimes \mathbb{F}_{2}\left\langle\widetilde{\mathbf{x}}_{0}, \widetilde{\mathbf{y}}_{0}\right\rangle$, and both $\widetilde{\mathbf{x}}_{0}$ and $\widetilde{\mathbf{y}}_{0}$ are fixed by the $\mathbb{Z} / 2$-action. 
Moduli spaces in $\widetilde{\mathcal{H}}^{\prime}$ with multiplicity 0 in the connected sum region correspond to moduli spaces in $\widetilde{\mathcal{H}}$, as before; it remains to show that we can choose an equivariant complex structure so that the moduli spaces in $\widetilde{\mathcal{H}}^{\prime}$ with multiplicity 1 in the connected sum region are empty. To this end, stretch the neck in the connected sum region. For large neck length, there is a fibered product description

$$
\mathcal{M}\left(\phi^{\prime}\right) \cong \mathcal{M}(\phi) \times_{\mathbb{D}^{2}} \mathcal{M}\left(\phi_{0}\right)
$$

just as before. Letting $p$ approach one of the $\alpha$-circles is not $\mathbb{Z} / 2$-equivariant. However, we can pinch the rectangle so that points on both $\widetilde{\alpha}_{0}$ and $\widetilde{\alpha}_{0}^{\prime}$ approach $p$ and so that the involution still exchanges $\widetilde{\alpha}_{0}$ and $\widetilde{\alpha}_{0}^{\prime}$. (This is the preimage of letting $p$ approach a point on $\alpha_{0} \subset S^{2}$.) This still has the effect that $\widetilde{u}_{0}^{-1}(p) \rightarrow \partial \mathbb{D}^{2}$, and so still implies that, after sufficient pinching, $\mathcal{M}\left(\phi^{\prime}\right)$ is empty.

Thus, we have shown that for appropriate choice of $\mathbb{Z} / 2$-equivariant almost complex structures we have an isomorphism of chain complexes over $\mathbb{F}_{2}[\mathbb{Z} / 2]$

$$
\widehat{C F K}\left(\widetilde{\mathcal{H}}^{\prime}\right) \simeq \widehat{C F K}(\widetilde{\mathcal{H}}) \otimes V,
$$

where $\mathbb{Z} / 2$ acts trivially on $V$.

Now we turn our attention to the $E_{\infty}$ page of the spectral sequence coming from $\widehat{C F K}(\widetilde{\mathcal{H}})^{\prime}$. For notational simplicity, let $\widetilde{H F K}^{*}\left(\widetilde{\mathcal{H}^{\prime}}\right)=H^{*}\left(\widehat{C F K}\left(\widetilde{\mathcal{H}^{\prime}}\right)\right)$, and similarly for $\widetilde{H F K}^{*}(\widetilde{\mathcal{H}})$. The existence of the $\mathbb{F}_{2}[\mathbb{Z} / 2]$ quasi-isomorphism (5.2) implies that the spectral sequence

$$
\widetilde{H F K^{*}}\left(\widetilde{\mathcal{H}^{\prime}}\right) \otimes \mathbb{F}_{2}\left[\theta, \theta^{-1}\right] \Rightarrow \theta^{-1} H F_{\mathbb{Z} / 2}\left(\widetilde{T}_{\alpha}^{\prime}, \widetilde{T}_{\beta}^{\prime}\right)
$$

splits as a direct sum of two identical spectral sequences,

$$
\widetilde{H F K}^{*}(\widetilde{\mathcal{H}}) \otimes \mathbb{F}_{2}\left[\theta, \theta^{-1}\right] \Rightarrow \theta^{-1} H F_{\mathbb{Z} / 2}\left(\widetilde{T}_{\alpha}, \widetilde{T}_{\beta}\right)
$$

In particular, $\theta^{-1} H F_{\mathbb{Z} / 2}\left(\widetilde{T}_{\alpha}^{\prime}, \widetilde{T}_{\beta}^{\prime}\right) \cong \theta^{-1} H F_{\mathbb{Z} / 2}\left(\widetilde{T}_{\alpha}, \widetilde{T}_{\beta}\right) \otimes V$ via a map of $\mathbb{F}_{2}\left[\theta, \theta^{-1}\right]$-modules that preserves Alexander gradings.

However, any Heegaard diagram $\mathcal{H}$ for $\left(S^{3}, K\right)$ is equivalent, after sufficiently many ordinary Heegaard moves and stabilizations of type 0-1-2-3, to a genus-0 Heegaard diagram $\mathcal{H}^{\prime \prime}$ admitting a localization isomorphism $\theta^{-1} H F_{\mathbb{Z} / 2}\left(\widetilde{T}_{\alpha}^{\prime \prime}, \widetilde{T}_{\beta}^{\prime \prime}\right) \cong \widetilde{H F K^{*}}\left(T_{\alpha}^{\prime \prime}, T_{\beta}^{\prime \prime}\right) \otimes \mathbb{F}_{2}\left[\theta, \theta^{-1}\right]$. This localization isomorphism also preserves Alexander gradings [Hen12, page 2144]. Using the previous argument to inductively split off copies of $V$, we conclude that the spectral sequence coming from $\widehat{C F K}(\mathcal{H})$ is

$$
\widetilde{H F K}(\widetilde{\mathcal{H}}) \otimes \mathbb{F}_{2}\left[\theta, \theta^{-1}\right] \Rightarrow H F_{\mathbb{Z} / 2}\left(T_{\alpha}, T_{\beta}\right) \cong \widetilde{H F K}(\mathcal{H}) \otimes \mathbb{F}_{2}\left[\theta, \theta^{-1}\right]
$$

and in particular, if $\mathcal{H}$ has a single pair of basepoints, is exactly

$$
\widehat{H F K}^{*}(\Sigma(K), \widetilde{K}) \otimes \mathbb{F}_{2}\left[\theta, \theta^{-1}\right] \Rightarrow \theta^{-1} H F_{\mathbb{Z} / 2}\left(\widetilde{T}_{\alpha}, \widetilde{T}_{\beta}\right) \cong \widehat{H F K}^{*}\left(S^{3}, K\right) \otimes \mathbb{F}_{2}\left[\theta, \theta^{-1}\right],
$$

where the last isomorphism preserves the absolute Alexander grading.

Proof of Corollary 1.15. Let $\mathcal{H}$ be a Heegaard diagram for $\left(S^{3}, K\right)$ with a single pair of basepoints and let $\widetilde{\mathcal{H}}$ be its double branched cover over the basepoints so that it is a Heegaard diagram for $(\Sigma(K), \widetilde{K})$. The involution $\tau$ on $\Sigma(K)$ induces an action on $\operatorname{Spin}^{\mathrm{c}}(\Sigma(K))$ which takes each $\operatorname{spin}^{c}$-structure to its conjugate and fixes only the unique spin structure $\mathfrak{s}_{0}$ [Gri06, page 1378], [Lev08, Remark 3.4]. Moreover, $\tau$ preserves the absolute Alexander grading [Lev08, Proposition 3.4]. Thus, the equivariant cochain complex $C F_{\mathbb{Z} / 2}\left(\widetilde{T}_{\alpha}, \widetilde{T}_{\beta}\right)$ splits along 
Alexander gradings and orbits of $\operatorname{spin}^{c}$-structures. Ergo the spectral sequence (1.6) restricts to spectral sequences

$$
\widehat{H F K}\left(\Sigma(K), \widetilde{K}, \mathfrak{s}_{0}, i\right) \otimes \mathbb{F}_{2}\left[\theta, \theta^{-1}\right] \Rightarrow \theta^{-1} H F_{\mathbb{Z} / 2}\left(\widetilde{T}_{\alpha}, \widetilde{T}_{\beta}, \mathfrak{s}_{0}, i\right)
$$

SO

$$
\operatorname{dim}\left(\widehat{H F K}\left(\Sigma(K), \widetilde{K}, \mathfrak{s}_{0}, i\right)\right) \geq \operatorname{rank}\left(\theta^{-1} H F_{\mathbb{Z} / 2}\left(\widetilde{T}_{\alpha}, \widetilde{T}_{\beta}, \mathfrak{s}_{0}, i\right)\right),
$$

where the latter rank is as an $\mathbb{F}_{2}\left[\theta, \theta^{-1}\right]$ module. It remains to check that

$$
\operatorname{rank}\left(\theta^{-1} H F_{\mathbb{Z} / 2}\left(\widetilde{T}_{\alpha}, \widetilde{T}_{\beta}, \mathfrak{s}_{0}, i\right)\right)=\operatorname{dim}\left(\widehat{H F K}\left(S^{3}, K, i\right)\right) .
$$

Grigsby showed that the absolute Alexander grading of an equivariant lift $\widetilde{\mathbf{x}} \in \widetilde{T}_{\alpha} \cap \widetilde{T}_{\beta}$ of a generator $\mathbf{x} \in T_{\alpha} \cap T_{\beta}$ is the same as the absolute Alexander grading of $\mathbf{x}$ [Gri06, Lemma 4.7] (see also [Lev08, Proposition 3.4]). Furthermore, localization isomorphisms for $(\Sigma(K), \widetilde{K})$ preserve absolute Alexander gradings and $\operatorname{spin}^{c}$ structures [Hen12, page 2144], which in light of the proof of Proposition Theorem 1.14 implies that the isomorphism $\theta^{-1} H_{\mathbb{Z} / 2}\left(\widetilde{T}_{\alpha}, \widetilde{T}_{\beta}\right) \simeq$ $\widehat{H F K}\left(S^{3}, K\right) \otimes \mathbb{F}_{2}\left[\theta, \theta^{-1}\right]$ does as well. The conclusion follows.

Proof of Theorem 1.16. The chain-level version of Theorem 1.16 is considerably simpler. Let $\mathcal{H}$ be a multi-pointed Heegaard diagram for $\left(S^{3}, K \cup A\right)$ so that exactly two basepoints $z_{A}$ and $w_{A}$ correspond to the axis $A$. Let $\widetilde{\mathcal{H}}$ be the double cover of $\mathcal{H}$ branched over $\left\{z_{A}, w_{A}\right\}$, $\mathcal{H}^{\prime}$ the result of doing an index 0-1-2-3 stabilization as on the left of Figure 2 to $\mathcal{H}$ at a basepoint $z$ on $K\left(\right.$ not $\left.z_{A}\right)$, and $\widetilde{\mathcal{H}}^{\prime}$ the double cover of $\mathcal{H}^{\prime}$ branched along $\left\{z_{A}, w_{A}\right\}$. We will show that for appropriate choice of almost complex structures there is a $\mathbb{Z} / 2$-equivariant chain isomorphism

$$
\widehat{C F L}\left(\widetilde{\mathcal{H}^{\prime}}\right) \simeq \widehat{C F L}(\widetilde{\mathcal{H}}) \otimes X
$$

where $X=\{x x, x y, y x, y y\}$ with action

$$
\tau(x x)=x x \quad \tau(x y)=y x \quad \tau(y x)=x y \quad \tau(y y)=y y .
$$

The theorem is a homology-level reinterpretation of this fact (and induction).

Since we are branching over $A$, not $K$, the diagram $\widetilde{\mathcal{H}}^{\prime}$ has two regions which look like the left of Figure 2, which are exchanged by $\tau$. Stretching the neck around both such regions decomposes $\widetilde{\mathcal{H}}^{\prime}$ as $\widetilde{\mathcal{H}} \cup\left(S^{2}, \alpha_{0}, \beta_{0},\left\{z_{0}, z_{0}^{\prime}\right\},\left\{w_{0}\right\}\right) \cup\left(S^{2}, \alpha_{0}, \beta_{0},\left\{z_{0}, z_{0}^{\prime}\right\},\left\{w_{0}\right\}\right)$. This induces the desired isomorphism of abelian groups (5.3) where, say, the $x y$ summand corresponds to choosing the generator $x$ in the first copy of $S^{2}$ and $y$ in the second copy of $S^{2}$. The same proof as in the non-equivariant case implies that this is a chain isomorphism; to ensure it is $\mathbb{Z} / 2$-equivariant we must move both basepoints $p$ close to the $\alpha$-circles, but this does not interfere with the argument. This proves the $\widehat{H F L}$ case.

For the case of $\widehat{H F K}$, there is an additional complication that Condition (ET-2) does not apply, so we do not know that we can compute $\widehat{H F K}$ using complex structures which are $\mathbb{Z} / 2$-equivariant. Thus, we are forced to work with the more complicated definition of the equivariant complex from Section 3. We may, however, assume that all of the complex structures and paths of complex structures are split over the stabilization regions (the two $S^{2}$ 's in the discussion above). Under this assumption, we still have a fiber product description of the moduli spaces, and taking the same $p \rightarrow \alpha$ limit implies that the differential on the equivariant complex for $\widetilde{\mathcal{H}}^{\prime}$ is again of the form $\widehat{C F L}_{\mathbb{Z} / 2}(\widetilde{\mathcal{H}}) \otimes X$. Further details are left to the reader. 
Proof of Corollary 1.19. The existence of the spectral sequence (1.17) immediately implies that

$$
2 \operatorname{dim}\left(\widehat{H F L}\left(S^{3}, \widetilde{K} \cup \widetilde{A}\right)\right) \geq \operatorname{dim}\left(\widehat{H F L}\left(S^{3}, K \cup A\right)\right)
$$

Our goal is to remove the factor of two in this inequality by proving that at least half the rank of $\widehat{H F L}\left(S^{3}, \widetilde{K} \cup \widetilde{A}\right) \otimes V_{1}$ dies in the spectral sequence.

Recall that if $\mathcal{H}$ is a Heegaard diagram for a $\operatorname{link} L=L_{1} \cup \cdots \cup L_{n}$ then the complex $\widehat{C F L}(\mathcal{H})$ has an Alexander multi-grading, $\left(A_{L_{1}}, \cdots, A_{L_{n}}\right)$, valued in integers or half-integers according to the parity of the linking numbers of certain combinations of components. The differential on $\widehat{C F L}(\mathcal{H})$ preserves this multigrading.

With this in mind, let $\mathcal{H}$ be a nice diagram for $K \cup A$ with one pair of basepoints on each component of the link. Let $\widetilde{\mathcal{H}}$ be the branched cover of $\mathcal{H}$ branched over the basepoints on $A$, so that $\widetilde{\mathcal{H}}$ is a nice Heegaard diagram for $\widetilde{K} \cup \widetilde{A}$ with two pairs of basepoints on $\widetilde{K}$ and one on $\widetilde{A}$. Let $\widetilde{H F L}(\widetilde{\mathcal{H}})$ denote the Heegaard Floer homology of the multi-pointed Heegaard $\operatorname{diagram} \widetilde{\mathcal{H}}$, so $\widetilde{H F L}(\widetilde{\mathcal{H}}) \cong \widehat{H F L}\left(S^{3}, \widetilde{K} \cup \widetilde{A}\right) \otimes V_{1}$.

We will concern ourselves only with the Alexander grading $A_{\widetilde{K}}$ associated to the component $\widetilde{K}$ of $\widetilde{K} \cup \widetilde{A}$, not the Alexander grading coming from the axis. The grading $A_{\widetilde{K}}$ is always a half-integer, say $\frac{2 k-1}{2}$. Write $\widetilde{H F L}\left(\widetilde{\mathcal{H}}, \frac{2 k-1}{2}\right)$ for the summand of $\widetilde{H F L}(\mathcal{H})$ spanned by

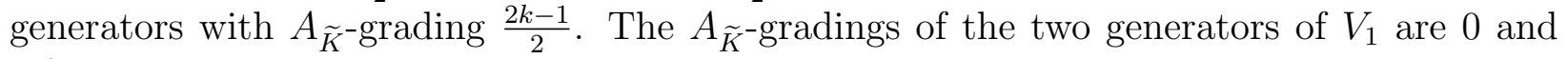
-1 , so

$$
\bigoplus_{k \text { even }} \widetilde{H F L}\left(\tilde{\mathcal{H}}, \frac{2 k-1}{2}\right) \simeq \underset{k \text { odd }}{\bigoplus} \widetilde{H F L}\left(\widetilde{\mathcal{H}}, \frac{2 k-1}{2}\right)
$$

The Heegaard diagram $\widetilde{\mathcal{H}}$ admits an equivariant complex structure achieving transversality, which by Proposition 4.3 may be used to construct $H F_{\mathbb{Z} / 2}\left(\widetilde{T}_{\alpha}, \widetilde{T}_{\beta}\right)$. Moreover, since the double branched cover involution $\tau_{\#}$ preserves the absolute Alexander multi-grading on $\widehat{C F L}(\widetilde{\mathcal{H}})[$ Hen15, Lemma 3.1], we see the spectral sequence (1.17) splits along the Alexander grading $A_{\widetilde{K}}$ :

$$
\widetilde{H F L}^{*}\left(\widetilde{\mathcal{H}}, \frac{2 k-1}{2}\right) \otimes \mathbb{F}_{2}\left[\theta, \theta^{-1}\right] \Rightarrow \theta^{-1} H F_{\mathbb{Z} / 2}\left(\widetilde{T}_{\alpha}, \widetilde{T}_{\beta}, \frac{2 k-1}{2}\right)
$$

However, if we let $\mathbf{x} \in T_{\alpha} \cap T_{\beta}$ be a generator in $C F\left(T_{\alpha}, T_{\beta}\right)$ and $\widetilde{\mathbf{x}}$ be the corresponding equivariant generator in $\widetilde{T}_{\alpha} \cap \widetilde{T}_{\beta}$, then $A_{\widetilde{K}}(\widetilde{\mathbf{x}})=2 A_{K}(\mathbf{x})-\frac{1}{2}=\frac{4 A_{k}(\mathbf{x})-1}{2}$ [Hen15, Lemma 3.7]. In particular, all equivariant generators lie in $A_{\widetilde{K}}$ gradings $i=\frac{2 k+1}{2}$ for even $k$, implying that $\theta^{-1} H F_{\mathbb{Z} / 2}\left(\widetilde{T}_{\alpha}, \widetilde{T}_{\beta}, \frac{2 k-1}{2}\right)=0$ when $k$ is odd. We conclude that half the rank of $\widehat{H F K}\left(S^{3}, \widetilde{K} \cup\right.$ $\widetilde{A}) \otimes V \otimes \mathbb{F}_{2}\left[\theta, \theta^{-1}\right]$ is lost in the spectral sequence (1.17), as desired.

5.4. Equivalence of the Hendricks and Lipshitz-Treumann spectral sequences. Given a surface $F$, together with a handle decomposition of $F$ with a single 0-handle and a single 2-handle, and $2 g$ 1-handles, bordered Floer homology [LOT08, LOT15] associates a differential algebra $\mathcal{A}(F)=\bigoplus_{i=-g}^{g} \mathcal{A}(F, i)$. Given a 3-dimensional cobordism $Y$ from $F_{1}$ to $F_{2}$, together with a framed arc $\gamma$ in $Y$ connecting the 0-handle in $F_{1}$ and the 0-handle in $F_{2}$, bordered Floer homology associates a $d g\left(\mathcal{A}\left(F_{1}\right), \mathcal{A}\left(F_{2}\right)\right)$-bimodule $\widehat{C F D A}(Y)$, welldefined up to quasi-isomorphism. (The bimodule $\widehat{C F D A}(Y)$ as defined in [LOT15] may, more generally, be an $A_{\infty}$-bimodule, but any $A_{\infty}$-bimodule is quasi-isomorphic to a $d g$ bimodule; alternatively, if one computes $\widehat{C F D A}(Y)$ from a nice Heegaard diagram then the higher 
operations on $\widehat{C F D A}(Y)$ vanish.) We will call $(Y, \gamma)$ an arced cobordism. If $\left(Y^{\prime}, \gamma^{\prime}\right)$ is an arced cobordism from $F_{2}$ to $F_{3}$ then $\widehat{C F D A}\left(Y \cup_{F_{2}} Y^{\prime}\right) \simeq \widehat{C F D A}\left(Y_{1}\right) \otimes^{L}{ }_{\mathcal{A}\left(F_{2}\right)} \widehat{C F D A}\left(Y_{2}\right)$, where $\otimes^{L}$ is the derived tensor product [LOT15, Theorem 12]. If $F_{1}=F_{2}$ then we can self-glue $(Y, \gamma)$ to get a closed 3-manifold with a framed knot. Doing framed surgery on the knot gives another 3-manifold $Y^{\circ}$, and the core of the surgery solid torus is a knot $K^{\circ}$ in $Y$. Then $\widehat{H F K}\left(Y^{\circ}, K^{\circ}\right) \cong H H(\widehat{C F D A}(Y))[$ LOT15, Theorem 14].

Given a $d g(A, A)$-bimodule $M$, one can define a $\mathbb{Z} / 2$-equivariant Hochschild homology of $M$ as follows. Fix a biprojective resolution $R$ of $A$. Then $H H(M)$ is the homology of $H C(M)=M \otimes R / \sim$ where for any $m \in M, a \in A$, and $r \in R$,

$$
m a \otimes r \sim m \otimes a r \quad \text { and } \quad a m \otimes r \sim m \otimes r a .
$$

Similarly, $H H\left(M \otimes^{L} M\right)$ is the homology of $H C\left(M \otimes^{L} M\right)=M \otimes R \otimes M \otimes R / \sim$ where

$$
a_{1} m a_{2} \otimes r \otimes a_{3} m^{\prime} a_{4} \otimes r^{\prime} \sim m \otimes a_{2} r a_{3} \otimes m^{\prime} \otimes a_{4} r^{\prime} a_{1}
$$

There is an action of $\mathbb{Z} / 2$ on $H C\left(M \otimes^{L} M\right)$ by

$$
\tau\left(m \otimes r \otimes m^{\prime} \otimes r^{\prime}\right)=m^{\prime} \otimes r^{\prime} \otimes m \otimes r .
$$

Define the $\mathbb{Z} / 2$-equivariant Hochschild complex and homology by

$$
\begin{aligned}
& H C_{\mathbb{Z} / 2}(M)=\operatorname{RHom}_{\mathbb{F}_{2}[\mathbb{Z} / 2]}\left(H C\left(M \otimes^{L} M\right), \mathbb{F}_{2}\right) \\
& H H_{\mathbb{Z} / 2}(M)=\operatorname{Ext}_{\mathbb{F}_{2}[\mathbb{Z} / 2]}\left(H C\left(M \otimes^{L} M\right), \mathbb{F}_{2}\right)=H\left(H C_{\mathbb{Z} / 2}(M)\right) .
\end{aligned}
$$

From its definition as an Ext group, $H_{\mathbb{Z} / 2}(M)$ inherits an action of $\operatorname{Ext}_{\mathbb{F}_{2}[\mathbb{Z} / 2]}\left(\mathbb{F}_{2}, \mathbb{F}_{2}\right) \cong$ $\mathbb{F}_{2}[\theta]$. There is also a filtration on $H C_{\mathbb{Z} / 2}(M)$ : explicitly, we can write

$$
H C_{\mathbb{Z} / 2}(M)=\left\{0 \rightarrow H C\left(M \otimes^{L} M\right)^{*} \stackrel{1+\tau}{\longrightarrow} H C\left(M \otimes^{L} M\right)^{*} \stackrel{1+\tau}{\longrightarrow} \cdots\right\},
$$

and then the horizontal filtration on this bicomplex gives a filtration on $H C_{\mathbb{Z} / 2}(M)$. The induced spectral sequence has $E_{1}$-page given by $H H\left(M \otimes^{L} M\right)^{*} \otimes \mathbb{F}_{2}[\theta]$ (In the language of [LT16], this is the ${ }^{v h} E$ spectral sequence.)

Lemma 5.4. The filtered quasi-isomorphism type of $\mathrm{HC}_{\mathbb{Z} / 2}(M)$ depends only on the quasiisomorphism type of the $d g$ bimodule $M$ and is independent of the choice of resolution $R$.

Proof. Given a quasi-isomorphism $f: M \rightarrow N$ there is an induced quasi-isomorphism

$$
f \otimes \mathbb{I} \otimes f \otimes \mathbb{I}:(M \otimes R \otimes M \otimes R) / \sim \rightarrow(N \otimes R \otimes N \otimes R) / \sim,
$$

and this induced map is a map of $\mathbb{F}_{2}[\mathbb{Z} / 2]$-modules. Further, given two resolutions $R$ and $R^{\prime}$ of $A$ and a quasi-isomorphism $g: R \rightarrow R^{\prime}$ there is an induced quasi-isomorphism

$$
\mathbb{I} \otimes g \otimes \mathbb{I} \otimes g:(M \otimes R \otimes M \otimes R) / \sim \rightarrow\left(M \otimes R^{\prime} \otimes M \otimes R^{\prime}\right) / \sim,
$$

which again is a map of $\mathbb{F}_{2}[\mathbb{Z} / 2]$-modules. The result follows.

Proof of Theorem 1.20. Fix a nice Heegaard diagram $\mathcal{H}_{Y}$ for $Y$. Recall the bordered Heegaard diagrams $\mathrm{AZ}(-F)$ and $\overline{\mathrm{AZ}}(F)$ associated to $F$ [LOT11, Section 4] which have the properties that:

- the result $\mathcal{H}_{\mathbb{I}}=\mathrm{AZ}(-F) \cup \overline{\mathrm{AZ}}(F)$ of gluing $\mathrm{AZ}(-F)$ to $\overline{\mathrm{AZ}}(F)$ along one boundary component is a diagram for the identity cobordism of $F$ [LOT11, Corollary 4.5], and

- the bimodule $\widehat{C F D A}\left(\mathcal{H}_{\mathbb{I}}\right)$ is a biprojective resolution of the diagonal $(\mathcal{A}(F), \mathcal{A}(F))$ bimodule $\mathcal{A}(F)$ [LT16, Proof of Proposition 4.1]. 
Further, the proofs of the pairing theorem and self-pairing theorem via nice diagrams [LOT15, Section 7] give an isomorphism

$$
\widehat{C F K}\left(\mathcal{H}_{Y} \cup \mathcal{H}_{\mathbb{I}} \cup \mathcal{H}_{Y} \cup \mathcal{H}_{\mathbb{I}} / \sim\right) \cong \widehat{C F D A}\left(\mathcal{H}_{Y}\right) \otimes \widehat{C F D A}\left(\mathcal{H}_{\mathbb{I}}\right) \otimes \widehat{C F D A}\left(\mathcal{H}_{Y}\right) \otimes \widehat{C F D A}\left(\mathcal{H}_{\mathbb{I}}\right) / \sim
$$

where the equivalence relation $\sim$ on the left hand side identifies corresponding boundary components, circularly; and this isomorphism respects the $\mathbb{Z} / 2$-actions. So, the result follows from Lemma 5.4, which says that we can use the right hand side to compute the equivariant Hochschild homology, and Corollary 1.13, which says that we can use the left hand side to compute the equivariant Floer cohomology.

5.5. Invariants of covering spaces. Consider a normal covering space $\pi: \widetilde{Y} \rightarrow Y$ of degree $N<\infty$, with deck group $H$. Given a singly-pointed, genus $g$ Heegaard diagram $\mathcal{H}$ for $Y$, there is a corresponding $N$-pointed Heegaard diagram $\widetilde{\mathcal{H}}=(\widetilde{\Sigma}, \widetilde{\boldsymbol{\alpha}}, \widetilde{\boldsymbol{\beta}}, \widetilde{z})$ for $\widetilde{Y}$ and a projection map $\widetilde{\mathcal{H}} \rightarrow \mathcal{H}$ [LL08, Section 2.2]: $\widetilde{\mathcal{H}}$ is simply the total preimage, under $\pi$, of $\mathcal{H}$. The group $H$ acts on $\widetilde{\mathcal{H}}$ and hence on $\operatorname{Sym}^{N g}(\widetilde{\Sigma} \backslash \widetilde{z})$. Given any nontrivial $h \in H$, generators $\mathbf{x}, \mathbf{y} \in \widetilde{T}_{\alpha} \cap \widetilde{T}_{\beta} \subset \operatorname{Sym}^{N g}(\Sigma)$, and homotopy class $\phi \in \pi_{2}(\mathbf{x}, \mathbf{y})$, if $\phi=h_{*}(\phi)$ then $\mu(\phi)$ is divisible by the order of $h$. So, Condition (ET-2') is satisfied, and hence a generic 1-parameter family of $H$-equivariant almost complex structures satisfies Hypothesis 4.4. So, by Proposition 4.5 we can define the Floer complex $\widehat{C F}(\widetilde{\mathcal{H}})=C F\left(T_{\alpha}, T_{\beta}\right)$ as a module over $\mathbb{F}_{2}[H]$. The group $H$ also acts on $\operatorname{Spin}^{\mathrm{c}}(Y)$, and the complex $\widehat{C F}(\widetilde{\mathcal{H}})=C F\left(T_{\alpha}, T_{\beta}\right)$, over $\mathbb{F}_{2}[H]$, decomposes according to $H$-orbits of $\operatorname{spin}^{c}$-structures,

$$
\widehat{C F}(\widetilde{\mathcal{H}})=\bigoplus_{\left[\mathfrak{s}_{0}\right] \in \operatorname{Spin}^{\mathrm{c}}(\widetilde{Y}) / H} \widehat{C F}\left(\widetilde{\mathcal{H}},\left[\mathfrak{s}_{0}\right]\right) \quad \text { where } \widehat{C F}\left(\widetilde{\mathcal{H}},\left[\mathfrak{s}_{0}\right]\right)=\bigoplus_{\mathfrak{s} \in H_{\mathfrak{s}_{0}}} \widehat{C F}(\widetilde{\mathcal{H}}, \mathfrak{s}) .
$$

Given a torsion $\operatorname{spin}^{c}$-structure $\mathfrak{s}_{0}$ on $\tilde{Y}, \widehat{C F}\left(\widetilde{\mathcal{H}},\left[\mathfrak{s}_{0}\right]\right)$ is a relatively $\mathbb{Z}$-graded chain complex over $\mathbb{F}_{2}[H]$, so we define

$$
\widehat{H F}_{H}\left(\widetilde{\mathcal{H}},\left[\mathfrak{s}_{0}\right]\right)=\operatorname{Ext}_{\mathbb{F}_{2}[H]}\left(\widehat{C F}\left(\widetilde{\mathcal{H}},\left[\mathfrak{s}_{0}\right]\right), \mathbb{F}_{2}\right) .
$$

The equivariant homology $\widehat{H F}_{H}\left(\widetilde{\mathcal{H}},\left[\mathfrak{s}_{0}\right]\right)$ inherits an action of the group cohomology $H^{*}(H)=$ $\operatorname{Ext}_{\mathbb{F}_{2}[H]}\left(\mathbb{F}_{2}, \mathbb{F}_{2}\right)$ of $H$.

Theorem 5.5. The quasi-isomorphism type of $\widehat{C F}\left(\widetilde{\mathcal{H}},\left[\mathfrak{s}_{0}\right]\right)$ over $\mathbb{F}_{2}[H]$ and, in particular, the equivariant Heegaard Floer cohomology $\widehat{H F}_{H}\left(\widetilde{\mathcal{H}},\left[\mathfrak{s}_{0}\right]\right)$, as a module over $H^{*}(H)$, is an invariant of the covering space $\pi$ and orbit of $\operatorname{spin}^{c}$-structures $\left[\mathfrak{s}_{0}\right]$.

Proof. By Proposition 4.5, $\widehat{C F}\left(\widetilde{\mathcal{H}},\left[\mathfrak{s}_{0}\right]\right)$ is quasi-isomorphic to the freed Floer complex $\widetilde{C F}\left(T_{\alpha}, T_{\beta}\right)$

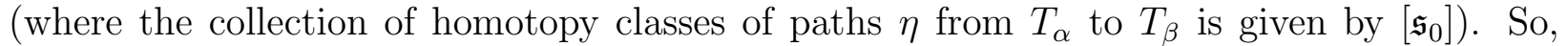
it follows from Proposition 3.28 that $\widehat{C F}\left(\widetilde{\mathcal{H}},\left[\mathfrak{s}_{0}\right]\right)$ is independent of the choices of almost complex structures used to define it and is invariant under equivariant Hamiltonian isotopies of $T_{\alpha}$ and $T_{\beta}$, up to quasi-isomorphism over $\mathbb{F}_{2}[H]$. It follows that the quasi-isomorphism type of $C F\left(\widetilde{\mathcal{H}},\left[\mathfrak{s}_{0}\right]\right)$ over $\mathbb{F}_{2}[H]$ is invariant under isotopies and handleslides of the $\alpha$ - and $\beta$-curves in $\mathcal{H}$ (see Lemma 5.1 and the proof of Corollary 1.10). Finally, it is trivially true that $C F\left(\widetilde{\mathcal{H}},\left[\mathfrak{s}_{0}\right]\right)$ is invariant under stabilizations near the basepoint (again, see the proof of Corollary 1.10). Since any two Heegaard diagrams for $Y$ are related by a sequence of isotopies, handleslides, and stabilizations near the basepoint, the result follows. 
The invariant $\widehat{C F}\left(\widetilde{\mathcal{H}},\left[\mathfrak{s}_{0}\right]\right)$ can, of course, be computed combinatorially starting from any nice diagram $\mathcal{H}$ for $Y$.

Proof of Theorem 1.22. The fact that the equivariant Floer homology is well-defined is Theorem 5.5. It follows from Corollary 1.13 that the equivariant Floer complex can be computed from a nice diagram. (We should note that the whole equivariant complex decomposes along the action of $\mathbb{Z} / 2$ on $\operatorname{Spin}^{\mathrm{c}}(\widetilde{Y})$.) If $\widetilde{Y} \rightarrow Y$ is induced by a $\mathbb{Z}$-cover, [LT16, Section 4.5] also gives an equivariant Floer homology and a spectral sequence $\widehat{H F}\left(\widetilde{Y}, \pi^{*}(\mathfrak{s})\right) \otimes V \otimes \mathbb{F}_{2}[\theta] \Rightarrow$ $\widehat{H F}_{\mathbb{Z} / 2}\left(\widetilde{Y}, \pi^{*}(\mathfrak{s})\right)$, via Hochschild homology and bordered Floer homology. Essentially the same argument as used to prove Theorem 1.20 implies that the spectral sequence from [LT16, Section 4.5] agrees with the spectral sequence from Theorem 5.5.

Remark 5.6. For $n$-fold cyclic covers induced by $\mathbb{Z}$ covers with $n$ arbitrary, one can also use bordered Floer homology to compute the spectral sequence (5.5), by essentially the same technique as in [LT16, Section 4.5].

\section{New SPeCtral Sequences from the BRAnChed DOUble COVER}

6.1. A concordance homomorphism from the $\mathbb{Z} / 2$-action on $\widehat{C F}(\Sigma(K))$. Fix a link $L \subset S^{3}$. Following Manolescu, [Man06], we can define a Heegaard diagram for the branched double cover $\Sigma(L)$ in terms of a bridge diagram for $L$. Specifically, choose a link diagram $D$ for $L$ and a decomposition of $D$ as a union of embedded $\operatorname{arcs} A_{i}, B_{i} \subset S^{3}, i=1, \ldots, n$, so that the $A_{i}$ (respectively $B_{i}$ ) arcs are pairwise disjoint and the $A_{i}$ arcs pass under the $B_{i}$ arcs. Let $\left\{p_{1}, \ldots, p_{2 n}\right\}=\partial\left(\bigcup_{i=1}^{n} A_{i}\right)=\partial\left(\bigcup_{i=1}^{n} B_{i}\right)$. Order the $A_{i}$ arcs so that $A_{n}$ and $B_{n}$ share the endpoint $p_{2 n}$. Let $\pi: \Sigma_{0} \rightarrow S^{2}$ be the double cover of $S^{2}$ branched along $\left\{p_{1}, \ldots, p_{2 n}\right\}$, and let $\alpha_{i}=\pi^{-1}\left(A_{i}\right)$ and $\beta_{i}=\pi^{-1}\left(B_{i}\right)$. Then

$$
\mathcal{H}=\left(\Sigma, \alpha_{1}, \ldots, \alpha_{n-1}, \beta_{1}, \ldots, \beta_{n-1}, z=p_{2 n}\right)
$$

is a pointed Heegaard diagram for $\Sigma(L)$, so $\widehat{H F}(\mathcal{H})=\widehat{H F}(\Sigma(L))$.

There is a $\mathbb{Z} / 2$-action on $\Sigma$, exchanging the two sheets of the branched cover, and this action preserves each $\alpha_{i}$, each $\beta_{i}$, and $z$. There is an induced $\mathbb{Z} / 2$-action $\tau$ : $\operatorname{Sym}^{n-1}(\Sigma \backslash$ $\{z\}) \rightarrow \operatorname{Sym}^{n-1}(\Sigma \backslash\{z\})$ preserving the Heegaard tori $T_{\alpha}$ and $T_{\beta}$. The fixed set of $\tau$ has several non-homeomorphic connected components. For example, if $n$ is odd, there is a component of $\operatorname{Fix}(\tau)$ containing $\pi^{-1}\left\{q_{1}, \ldots, q_{(n-1) / 2}\right\}$ for any points $q_{1}, \ldots, q_{(n-1) / 2} \in\left(S^{2} \backslash\right.$ $\left.\left\{p_{1}, \ldots, p_{2 n}\right\}\right)$; this component also contains, for instance, $p_{1}, p_{1}, q_{3}, \ldots, q_{(n-1) / 2}$. Given a subset $S \subset\left\{p_{1}, \ldots, p_{2 n}\right\}$ consisting of $n-1$ (distinct) points, $\pi^{-1}(S)$ also has cardinality $n-1$, and is an isolated point in $\operatorname{Fix}(\tau)$. Let

$$
D=\left\{\pi^{-1}(S)\left|S \subset\left\{p_{1}, \ldots, p_{2 n}\right\},\right| S \mid=n-1\right\} \subset \operatorname{Fix}(\tau)
$$

be the set of points of this form; we will call $D$ the discrete part of $\operatorname{Fix}(\tau)$.

Lemma 6.1. The $\tau$-fixed parts of $T_{\alpha}$ and $T_{\beta}$ satisfy $T_{\alpha}^{f i x}, T_{\beta}^{f i x} \subset D$ so, in particular, $T_{\alpha}^{f i x}$ and $T_{\beta}^{f i x}$ are finite sets of points. Further, $\left|T_{\alpha}^{f i x} \cap T_{\beta}^{f i x}\right|=2^{|L|-1}$, where $|L|$ denotes the number of components of $L$.

Proof. Since $\tau$ preserves each $\alpha$-circle set-wise, if $\mathbf{x}=\left\{x_{1}, \ldots, x_{n-1}\right\} \in T_{\alpha}$ is fixed by $\tau$ then each $x_{i}$ must be fixed by $\tau$, so $\mathbf{x}$ is in $D$. The same argument applies to $T_{\beta}$. For the second statement, consider the graph $G$ with one vertex for each $p_{i}$ and an edge for each $A_{i}$ and $B_{i}$, connecting the arc's endpoints. Let $G^{\prime}$ be the result of deleting the edges $A_{n}$ and $B_{n}$ 
from $G$. Then $G$ is homeomorphic to $L$, and so consists of $|L|$ cycles, and $G^{\prime}$ consists of an interval, a point, and $|L|-1$ cycles. An element of $T_{\alpha}^{f x} \cap T_{\beta}^{f x}$ is a subset of the vertices of $G^{\prime}$ of size $n-1$ containing exactly one endpoint of each $A_{i}$ and each $B_{i}, 1 \leq i \leq n-1$. There is one way of choosing such a subset for the interval, and two ways for each cycle, giving $2^{|L|-1}$ choices in all.

Convention 6.2. For the rest of this section, let $\eta$ denote the set of torsion $\operatorname{spin}^{c}$-structures on $\Sigma(L)$, viewed as a collection of homotopy classes of paths from $T_{\alpha}$ to $T_{\beta}$ (see Section 3.1). By $\widehat{C F}(\Sigma(L))$ and $C F^{-}(\Sigma(L))$ we mean $\bigoplus_{\mathfrak{s} \in \eta} \widehat{C F}(\Sigma(L), \mathfrak{s})$ and $\bigoplus_{\mathfrak{s} \in \eta} C F^{-}(\Sigma(L), \mathfrak{s})$, respectively.

The tuple $\left(\operatorname{Sym}^{n-1}(\Sigma \backslash\{z\}), T_{\alpha}, T_{\beta}, \tau\right)$ satisfies Hypothesis 3.2, so we can define the freed Floer complex $\widetilde{C F}(\Sigma(K)):=\widetilde{C F}\left(T_{\alpha}, T_{\beta}\right)$ and the equivariant Floer homology $\widehat{H F}_{\mathbb{Z} / 2}(\Sigma(K))=$ $\operatorname{Ext}_{\mathbb{F}_{2}[\mathbb{Z} / 2]}\left(\widetilde{C F}(\Sigma(K)), \mathbb{F}_{2}\right)$. Further, by Lemma 6.1, there are no non-constant holomorphic disks contained in the fixed set (since such disks would have to be contained in the discrete set $D$ ), so Hypothesis 4.1 is satisfied and hence, by Proposition 4.3, we can use a generic 1-parameter family of $\mathbb{Z} / 2$-equivariant almost complex structures to define $\widehat{C F}(\Sigma(K))$ over $\mathbb{F}_{2}[\mathbb{Z} / 2]$, and compute $\widehat{H F}_{\mathbb{Z} / 2}(\Sigma(K))$ as $\operatorname{Ext}_{\mathbb{F}_{2}[\mathbb{Z} / 2]}\left(\widehat{C F}(\Sigma(K)), \mathbb{F}_{2}\right)$. Of course, $\widetilde{C F}(\Sigma(K))$ and $\widehat{C F}(\Sigma(K))$ are quasi-isomorphic over $\mathbb{F}_{2}[\mathbb{Z} / 2]$; so for computations, it is more convenient to use $\widehat{C F}(\Sigma(K))$, although, in the some of the proofs, it is necessary to use $\widetilde{C F}(\Sigma(K))$. Let $\widehat{C F}_{\mathbb{Z} / 2}(\Sigma(K))$ denote $\mathrm{RHom}_{\mathbb{F}_{2}[\mathbb{Z} / 2]}\left(\widehat{C F}(\Sigma(K)), \mathbb{F}_{2}\right)$; its homology is $\widehat{H F}_{\mathbb{Z} / 2}(\Sigma(K))$.

Next we identify the localized equivariant cohomology:

Proposition 6.3. The localized equivariant Floer cohomology $\theta^{-1} \widehat{H F}_{\mathbb{Z} / 2}(\Sigma(L))$ is isomorphic to $\left(\mathbb{F}_{2} \oplus \mathbb{F}_{2}\right)^{|L|-1} \otimes \mathbb{F}_{2}\left[\theta, \theta^{-1}\right]$. In particular, there is a spectral sequence

$$
\widehat{H F}^{*}(\Sigma(L)) \otimes \mathbb{F}_{2}\left[\theta, \theta^{-1}\right] \Rightarrow\left(\mathbb{F}_{2} \oplus \mathbb{F}_{2}\right)^{|L|-1} \otimes \mathbb{F}_{2}\left[\theta, \theta^{-1}\right] .
$$

Proof. Roughly, the result follows from Seidel-Smith's localization theorem [SS10]. More precisely, it follows from the fact that the proof of their localization theorem [SS10, Theorem 20] only uses stable normal triviality on the components of the fixed set which intersect the Lagrangian submanifolds. By Lemma 6.1, these components are points, for which stable normal triviality is obvious.

The spectral sequence (6.4) is the spectral sequence (1.23) mentioned in the introduction. As promised in the introduction, it is an invariant of based links:

Proof of Theorem 1.24. As usual, we will actually show that the quasi-isomorphism type of $\widehat{C F}(\Sigma(L))$ over $\mathbb{F}_{2}[\mathbb{Z} / 2]$, or equivalently the quasi-isomorphism type of $\widehat{C F}(\Sigma(L))$ over $\mathbb{F}_{2}[\mathbb{Z} / 2]$, is an invariant of based links. Note that if two based links $L$ and $L^{\prime}$ containing the same basepoint $p$ are isotopic, then we may choose the entire isotopy to fix the basepoint $p$. Therefore, we do not need to prove invariance under changing which point $p_{2 n}$ is distinguished (or, equivalently, which pair of $\operatorname{arcs} A_{n}, B_{n}$ is deleted). Thus, we must prove invariance under:

- Changes of almost complex structure; and

- Bridge moves, i.e., isotopies of the $A_{i}$ and $B_{i}$, handleslides among the $A_{i}$ and $B_{i}$, and stabilizations (Figure 13).

For stabilizations, we will work with $\widehat{C F}(\Sigma(L))$; for the other moves, we will work with $\widetilde{C F}(\Sigma(L))$. 


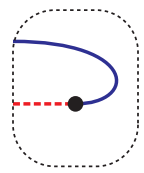

(a)

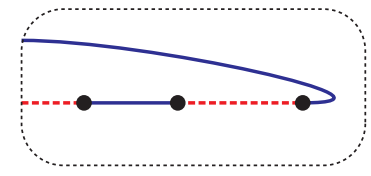

(b)

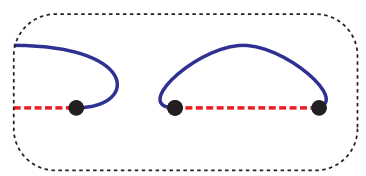

(c)

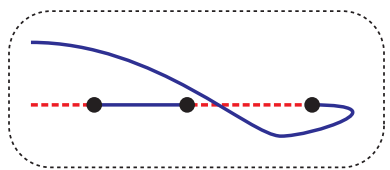

(d)

FiguRE 3. Stabilization and a skein sequence for the branched double cover. (a) A piece of a bridge diagram. $A_{i}$ is red and dashed and $B_{i}$ is blue and solid. The double cover of this diagram is $\mathcal{H}$. (b) A stabilization of the bridge diagram. The double cover of this diagram is $\mathcal{H}_{1}$. (c) The disjoint union of the bridge diagram with an unknot. The double cover of this diagram is $\mathcal{H}_{0}$. (d) A Reidemeister I move applied to the stabilization. The double cover of this diagram is $\mathcal{H}^{\prime}$. Diagrams (b) and (c) are obtained from (d) by unoriented skein moves.

Changes of almost complex structure induce quasi-isomorphisms over $\mathbb{F}_{2}[\mathbb{Z} / 2]$ by Proposition 3.23. Isotopies of the $A_{i}$ and $B_{i}$ give equivariant isotopies of the Lagrangians, and hence induce quasi-isomorphisms over $\mathbb{F}_{2}[\mathbb{Z} / 2]$ by Proposition 3.24. Handleslides among the $A_{1}, \ldots, A_{n-1}$ (respectively $B_{1}, \ldots, B_{n-1}$ ) give Hamiltonian isotopic Heegaard tori, by Perutz's work [Per08]. Further, since the fixed set of the $\mathbb{Z} / 2$ action is discrete, no nontrivial holomorphic disks are contained in the fixed set, so there are equivariant almost complex structures achieving equivariant transversality; see Point (ET-1) in Section 4. Note that the relevant top class is represented by fixed generators. Thus, by Proposition 3.25, handleslides induce equivariant quasi-isomorphisms. Handlesliding $A_{n}$ (respectively $B_{n}$ ) over one of the other $A_{i}$ (respectively $B_{i}$ ) has no effect on the complex at all. Since $S^{2} \backslash A_{n}$ is connected, we may trade handleslides of $A_{i}$ over $A_{n}$ for handlesliding $A_{i}$ over all the other $A$-arcs, and similarly for handlesliding $B_{i}$ over $B_{n}$.

To prove stabilization invariance, consider the diagrams shown in Figure 3. That is, let $\mathcal{H}$ be the branched double cover of a bridge diagram and let $\mathcal{H}_{1}$ be the branched double cover of the result of stabilizing the bridge diagram. The stabilization occurs near one of the points $p_{i}$. Since we have already verified handleslide invariance, we may assume that $p_{i}$ is in the same region as $p_{2 n}$, i.e., there is an arc from $p_{i}$ to $p_{2 n}$ which is disjoint from the $A_{i}$ and $B_{i}$. Let $\mathcal{H}_{0}$ be the connected sum of $\mathcal{H}$ and a 1-bridge diagram for the unknot, where the connected sum occurs in the region adjacent to $p_{i}$ and $p_{2 n}$. (So, $\mathcal{H}_{0}$ is a diagram for a link with a small unknot component.) Let $\mathcal{H}^{\prime}$ be the result of applying a Reidemeister I move to the new pair of arcs in $\mathcal{H}_{1}$. The motivation for this notation is that the underlying links for $\mathcal{H}_{0}$ and $\mathcal{H}_{1}$ are the zero- and one-resolutions of the crossing introduced by the Reidemeister I move in $\mathcal{H}^{\prime}$. (Here, we are using the convention of zero- and one-resolutions from [OSz05], which is opposite to Khovanov's [Kho00].) There is a skein triangle

$$
\cdots \rightarrow \widehat{H F}\left(\mathcal{H}^{\prime}\right) \stackrel{f}{\longrightarrow} \widehat{H F}\left(\mathcal{H}_{0}\right) \stackrel{g}{\longrightarrow} \widehat{H F}\left(\mathcal{H}_{1}\right) \rightarrow \widehat{H F}\left(\mathcal{H}^{\prime}\right) \rightarrow \ldots
$$

Note that:

(1) The diagram $\mathcal{H}_{0}$ represents $\Sigma(L) \#\left(S^{2} \times S^{1}\right)$; the diagrams $\mathcal{H}, \mathcal{H}^{\prime}$, and $\mathcal{H}_{1}$ all represent $\Sigma(L)$. 
(2) There are obvious, $\mathbb{Z} / 2$-equivariant isomorphisms

$$
\widehat{C F}\left(\mathcal{H}_{0}\right) \cong \widehat{C F}(\mathcal{H}) \otimes \widehat{C F}\left(S^{2} \times S^{1}\right) \cong \widehat{C F}(\mathcal{H}) \otimes \mathbb{F}_{2}\left\langle\Theta_{\text {top }}, \Theta_{\text {bot }}\right\rangle
$$

where the $\Theta_{\text {top }}$ (respectively $\Theta_{b o t}$ ) is the top-graded (respectively bottom-graded) generator of $\widehat{H F}\left(S^{2} \times S^{1}\right)$. (In particular, the $H_{1} /$ tors-action on $\widehat{H F}\left(S^{2} \times S^{1}\right)$ sends $\Theta_{\text {top }}$ to $\Theta_{b o t}$ and sends $\Theta_{b o t}$ to 0.$)$

(3) We already know that the quasi-isomorphism type of $\widehat{C F}$ over $\mathbb{F}_{2}$ is an invariant of $\Sigma(L)$; we are trying to prove that these maps respect the $\mathbb{F}_{2}[\mathbb{Z} / 2]$-module structure. Non-equivariant invariance implies that the long exact sequence (6.5) splits as

$$
0 \rightarrow \widehat{H F}\left(\mathcal{H}^{\prime}\right) \rightarrow \widehat{H F}\left(\mathcal{H}_{0}\right) \rightarrow \widehat{H F}\left(\mathcal{H}_{1}\right) \rightarrow 0 .
$$

(4) The map $g$ sends the kernel of the $H_{1}\left(S^{2} \times S^{1}\right)$-action to 0 , and $\widehat{H F}(\mathcal{H}) \otimes \mathbb{F}_{2}\left\langle\Theta_{\text {top }}\right\rangle$ isomorphically to $\widehat{H F}\left(\mathcal{H}_{1}\right)$.

The map $g$ is given by counting holomorphic triangles, and hence respects the $\mathbb{F}_{2}[\mathbb{Z} / 2]$-module structure. Thus, the composition

$$
\widehat{C F}(\mathcal{H}) \rightarrow \widehat{C F}\left(\mathcal{H}_{0}\right) \rightarrow \widehat{C F}\left(\mathcal{H}_{1}\right),
$$

where the first map sends $\widehat{C F}(\mathcal{H})$ to $\widehat{C F}(\mathcal{H}) \otimes \mathbb{F}_{2}\left\langle\Theta_{\text {top }}\right\rangle$, is an $\mathbb{F}_{2}[\mathbb{Z} / 2]$-equivariant quasiisomorphism.

In the special case that $K$ is a knot, we extract a new numerical invariant. Before localizing we have the spectral sequence

$$
\widehat{H F}^{*}(\Sigma(K)) \otimes \mathbb{F}_{2}[\theta] \Rightarrow \widehat{H F}_{\mathbb{Z} / 2}(\Sigma(K)) .
$$

Since $\theta^{-1} \widehat{H F}_{\mathbb{Z} / 2}(\Sigma(K)) \cong \mathbb{F}_{2}\left[\theta, \theta^{-1}\right]$, there is a (non-canonical) submodule of $\widehat{H F}_{\mathbb{Z} / 2}(\Sigma(K))$ isomorphic to $\mathbb{F}_{2}[\theta]$. Also, $\widehat{H F}_{\mathbb{Z} / 2}(\Sigma(K))$ inherits an absolute $\mathbb{Q}$-grading from $\widehat{C F}(\mathcal{H})$. We use the convention that multiplication by $\theta$ raises the Maslov grading by one (so that the equivariant differential on $\widehat{C F}_{\mathbb{Z} / 2}(\Sigma(K))$ raises the Maslov grading by one), and for $x \in$ $\widehat{C F}^{*}(\Sigma(K)), \operatorname{gr}\left(x \otimes \theta^{0}\right)=\operatorname{gr}(x)$. Under this convention, we let $q_{\tau}(K)$ be twice the minimum grading of an element of the tower $\mathbb{F}_{2}[\theta]$. More precisely we set:

$$
q_{\tau}(K)=2 \min \left\{q \mid \exists[x] \in \widehat{H F}_{\mathbb{Z} / 2}(\Sigma(K)), \operatorname{gr}([x])=q, \theta^{n}[x] \neq 0 \forall n\right\}
$$

As a trivial example, if $K$ is the unknot then $q_{\tau}(K)=0$. Similarly, if $K$ is alternating then $\Sigma(K)$ is an $L$-space, implying that $\widehat{H F}\left(\Sigma(K), \mathfrak{s}_{0}\right) \simeq \mathbb{F}_{2}$ and therefore $\widehat{H F}^{*}\left(\Sigma(K), \mathfrak{s}_{0}\right) \otimes \mathbb{F}_{2}[\theta] \simeq$ $\mathbb{F}_{2}[\theta]$. The induced map $\tau^{*}$ on $\widehat{H F}^{*}\left(\Sigma(K), \mathfrak{s}_{0}\right)$ must be the identity, so in the spectral sequence $\widehat{H F}^{*}\left(\Sigma(K), \mathfrak{s}_{0}\right) \Rightarrow \widehat{H F}_{\mathbb{Z} / 2}(\Sigma(K))$, we see that the differential $d_{1}=\theta\left(1+\tau^{*}\right)$ is zero. All further induced differentials are trivial for grading reasons, so $\widehat{H F}^{*}\left(\Sigma(K), \mathfrak{s}_{0}\right) \otimes \mathbb{F}_{2}[\theta] \simeq \widehat{H F}_{\mathbb{Z} / 2}(\Sigma(K))$, implying that $q_{\tau}(K)=2 d\left(\Sigma(K), \mathfrak{s}_{0}\right)=\delta(K)$.

Indeed, because $\delta(K)$ is always an integer, [MO07, Section 2], we see that $q_{\tau}(K)$ is as well.

We have the following graded refinement of Theorem 1.24

Proposition 6.7. The action of $\tau$ and the quasi-isomorphisms from Theorem 1.24 respect the absolute Maslov grading on $\widehat{C F}(\Sigma(K))$. In particular, the number $q_{\tau}(K)$ is a knot invariant. 
Proof. First, to see that $\tau_{\#}$ preserves the absolute Maslov grading, note that the mapping cylinder of $\tau$ is a rational homology cobordism from $\Sigma(K)$ to itself with the property that the cobordism map $\widehat{C F}(\Sigma(K)) \rightarrow \widehat{C F}(\Sigma(K))$ is $\tau_{\#}$. Such maps preserve the absolute Maslov grading [OSz06, Theorem 7.1]. (Alternatively, see the proof of Proposition 7.7.)

The quasi-isomorphisms induced by isotopies of the $A_{i}$ and $B_{i}$ and by handleslides can be thought of as maps induced by Heegaard moves on the branched double cover of the Heegaard diagram, which preserve the absolute Maslov grading; similarly, changes of complex structure induce grading-preserving isomorphisms. For stabilization invariance, recall that since only $\operatorname{spin}^{c}$-structures $\mathfrak{s}$ with $c_{1}(\mathfrak{s})=0$ persist past the first page of the spectral sequence (6.4), it suffices to prove that stabilization induces grading-preserving maps for those $\operatorname{spin}^{c}$-structures. Recall that the stabilization map is a composition

$$
\widehat{H F}(\Sigma(L)) \rightarrow \widehat{H F}\left(\Sigma(L) \#\left(S^{1} \times S^{2}\right)\right) \stackrel{g}{\rightarrow} \widehat{H F}(\Sigma(L))
$$

where the first map is inclusion of the summand $\widehat{H F}(\Sigma(L)) \otimes \mathbb{F}_{2}\left\langle\Theta_{\text {top }}\right\rangle \subset \widehat{H F}(\Sigma(L)) \otimes$ $\mathbb{F}_{2}\left\langle\Theta_{\text {top }}, \Theta_{\text {bot }}\right\rangle$ and the second map is from the surgery exact sequence, and in particular is induced by a two-handle cobordism. By construction the first map of Formula (6.8) raises the absolute Maslov grading by $\frac{1}{2}$. For the second map, let $W$ be the two-handle surgery cobordism from $\Sigma(L) \#\left(S^{1} \times S^{2}\right)$ to $\Sigma(L)$. The cobordism map $g=F_{W}$ breaks up as a sum of maps $\Sigma_{\mathfrak{t} \in \operatorname{Spin}^{\mathrm{c}}(W)} F_{W, \mathfrak{t}}$. Let $\mathfrak{s}$ be a $\operatorname{spin}^{c}$-structure on $\Sigma(L)$, and let $\mathfrak{s}_{0}$ be the torsion $\operatorname{spin}^{c}$-structure on $S^{1} \times S^{2}$. Let $\mathfrak{t}$ be the unique $\operatorname{spin}^{c}$-structure on $W$ restricting to $\mathfrak{s}^{2} \mathfrak{s}_{0}$. Then

$$
F_{W, \mathfrak{t}}: \widehat{H F}\left(\Sigma(L) \#\left(S^{1} \times S^{2}\right), \mathfrak{s} \# \mathfrak{s}_{0}\right) \rightarrow \widehat{H F}(\Sigma(L), \mathfrak{s})
$$

is the only component of $F_{W}$ landing in $\widehat{H F}(\Sigma(L), \mathfrak{s})$. Since $c_{1}(\mathfrak{s})=0$, using the absolute Maslov grading formula for cobordism maps we see that $F_{W, \mathfrak{t}}$ lowers the absolute Maslov grading by $\frac{1}{2}$ [OSz06, Theorem 7.1]. Thus overall our stabilization maps preserve the absolute Maslov grading.

More is true:

Proposition 6.9. The number $q_{\tau}(K)$ is a smooth concordance invariant.

Before proving Proposition 6.9 we must develop the cobordism maps somewhat.

Lemma 6.10. Given an oriented cobordism $T$ from a link $L_{0}$ to a link $L_{1}$ there is a map of equivariant Floer complexes $\widehat{C F}_{\mathbb{Z} / 2}\left(\Sigma\left(L_{1}\right)\right) \rightarrow \widehat{C F}_{\mathbb{Z} / 2}\left(\Sigma\left(L_{0}\right)\right)$ so that:

- The induced map on the $E^{1}$-page of the spectral sequence associated to the $\theta$-power filtration is

$$
\left(\hat{F}_{\Sigma(T)}^{*} \otimes \mathbb{I}\right): \widehat{H F}^{*}\left(\Sigma\left(L_{1}\right)\right) \otimes \mathbb{F}_{2}[\theta] \rightarrow \widehat{H F}^{*}\left(\Sigma\left(L_{0}\right)\right) \otimes \mathbb{F}_{2}[\theta]
$$

where $\hat{F}_{\Sigma(T)}$ is the Ozsváth-Szabó cobordism map associated to $\Sigma(T)$, and

- The map $\widehat{C F}_{\mathbb{Z} / 2}\left(\Sigma\left(L_{1}\right)\right) \rightarrow \widehat{C F}_{\mathbb{Z} / 2}\left(\Sigma\left(L_{0}\right)\right)$ has the same effect on gradings as the dual of the Ozsváth-Szabó cobordism map $\hat{F}_{\Sigma(T)}$.

Proof. Any cobordism of links $T \subset[0,1] \times S^{3}$ from $L_{0}$ to $L_{1}$ can be viewed as a sequence of:

- Bridge moves,

- Births / deaths, i.e., creation or annihilation of a 1-bridge unknot disjoint from the rest of the bridge diagram, and

- Saddles, as shown in Figure 4. 

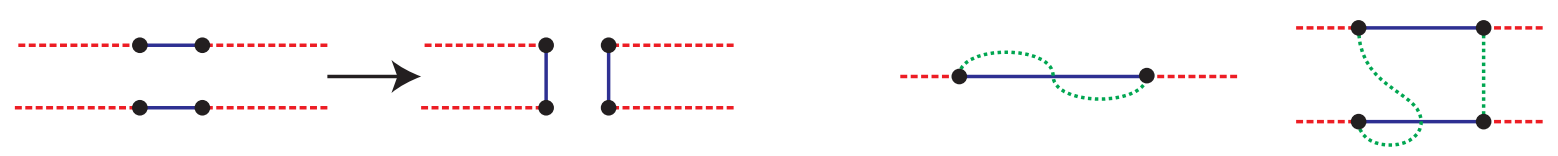

Figure 4. A saddle cobordism. Left: the cobordism itself. Right: the perturbations used in the triple diagram for the triangle map.

(See also [Wal09].) We can also assume that the births and deaths occur in a region adjacent to the basepoint $p_{2 n}$.

As we saw in Theorem 1.24, bridge moves induce quasi-isomorphisms of $\widetilde{C F}(\Sigma(L))$, or equivalently $\widehat{C F}(\Sigma(L))$, over $\mathbb{F}_{2}[\mathbb{Z} / 2]$; consequently, they induce maps of $\widehat{C F}_{\mathbb{Z} / 2}(\Sigma(L))$ over $\mathbb{F}_{2}[\theta]$, and it is straightforward to check that these maps satisfy the conditions of the lemma.

In the branched double cover, births correspond to 1-handles, deaths to 3-handles, and saddles to 2-handles. The Ozsváth-Szabó cobordism map associated to a 1-handle attachment is $x \mapsto x \otimes \Theta_{t o p}$, where $\Theta_{\text {top }}$ is the top-graded generator of $\widehat{C F}\left(S^{2} \times S^{1}\right)$ and the tensor product refers to the Künneth isomorphism $\widehat{C F}(\Sigma(K \amalg U)) \cong \widehat{C F}(\Sigma(K)) \otimes_{\mathbb{F}_{2}} \widehat{C F}\left(S^{2} \times S^{1}\right)$ [OSz06, Section 4.3]. (Here, for the chain-level statement, it is important that the new unknot component is disjoint from the other components of the bridge diagram, and it is convenient that it is adjacent to $p_{2 n}$.) Similarly, the Ozsváth-Szabó cobordism map associated to a death is $x \otimes \Theta_{\text {top }} \mapsto 0, x \otimes \Theta_{\text {bot }} \mapsto x$, where $\Theta_{b o t}$ is the bottom-graded generator of $\widehat{C F}\left(S^{2} \times S^{1}\right)$ [OSz06, Section 4.3]. Both of these maps are obviously $\mathbb{Z} / 2$-equivariant, and so give maps to equivariant complexes.

For saddles, there is a corresponding knot $C \subset \Sigma\left(L_{0}\right)$ and the Ozsváth-Szabó cobordism map is defined by considering a Heegaard triple diagram $(\Sigma, \boldsymbol{\alpha}, \boldsymbol{\beta}, \boldsymbol{\gamma})$ subordinate to $C$ and counting holomorphic triangles with one input the top-graded generator of $\widehat{H F}(\Sigma, \boldsymbol{\beta}, \boldsymbol{\gamma}) \cong$ $\left(\mathbb{F}_{2} \oplus \mathbb{F}_{2}\right)^{\otimes(n-2)}[$ OSz06, Section 4.1].

Consider the bridge triple diagram for the saddle shown on the right of Figure 4. This diagram consists of three sets of arcs $\left\{A_{i}\right\},\left\{B_{i}\right\}$, and $\left\{C_{i}\right\}$, so that $\left(\left\{A_{i}\right\},\left\{B_{i}\right\}\right)$ is a diagram for $L_{0},\left(\left\{A_{i}\right\},\left\{C_{i}\right\}\right)$ is a diagram for $L_{1}$, the result of the saddle move, and $\left(\left\{B_{i}\right\},\left\{C_{i}\right\}\right)$ is a diagram for an $(n-1)$-component unlink. If $B_{i}$ is not involved in the saddle move then we choose $C_{i}$ to be a small perturbation of $B_{i}$ intersecting $B_{i}$ at the two endpoints and one interior point, so that there is a bigon from each of the two endpoints to the middle point. For the two $B_{i}$ involved in the saddle move, we do a perturbation as shown, so that, in particular, one of the corresponding $C_{i}$ intersects one of the $B_{i}$ at an interior point. Let $(\Sigma, \boldsymbol{\alpha}, \boldsymbol{\beta}, \boldsymbol{\gamma}, z)$ denote the Heegaard triple diagram which is the branched double cover of this bridge triple diagram. The perturbations are chosen so that in the branched double cover, the top-graded generator for $\widehat{H F}(\Sigma, \boldsymbol{\beta}, \boldsymbol{\gamma})$ is represented by the sum of all $2^{n-2}$ fixed generators. In particular, all of the fixed generators for $(\Sigma, \beta, \gamma)$ have the same grading. By stabilization invariance, we may also assume that none of the arcs involved in the saddle is distinguished.

Now, as in the proof of Proposition 3.25, we can define a $\mathbb{Z} / 2 \mathbb{Z}$-equivariant triangle map betweeen the freed Floer complexes $\widetilde{C F}\left(\Sigma\left(L_{0}\right)\right) \rightarrow \widetilde{C F}\left(\Sigma\left(L_{1}\right)\right)$. We claim that, for suitable choices of almost complex structures, this map is induced by a map of ordinary (not freed) Floer complexes $\widehat{C F}\left(\Sigma\left(L_{0}\right)\right) \rightarrow \widehat{C F}\left(\Sigma\left(L_{1}\right)\right)$. The argument is similar to the proof of Proposition 4.3. As in Proposition 4.3, to define the freed Floer complexes for $(\Sigma, \boldsymbol{\alpha}, \boldsymbol{\beta})$ and 

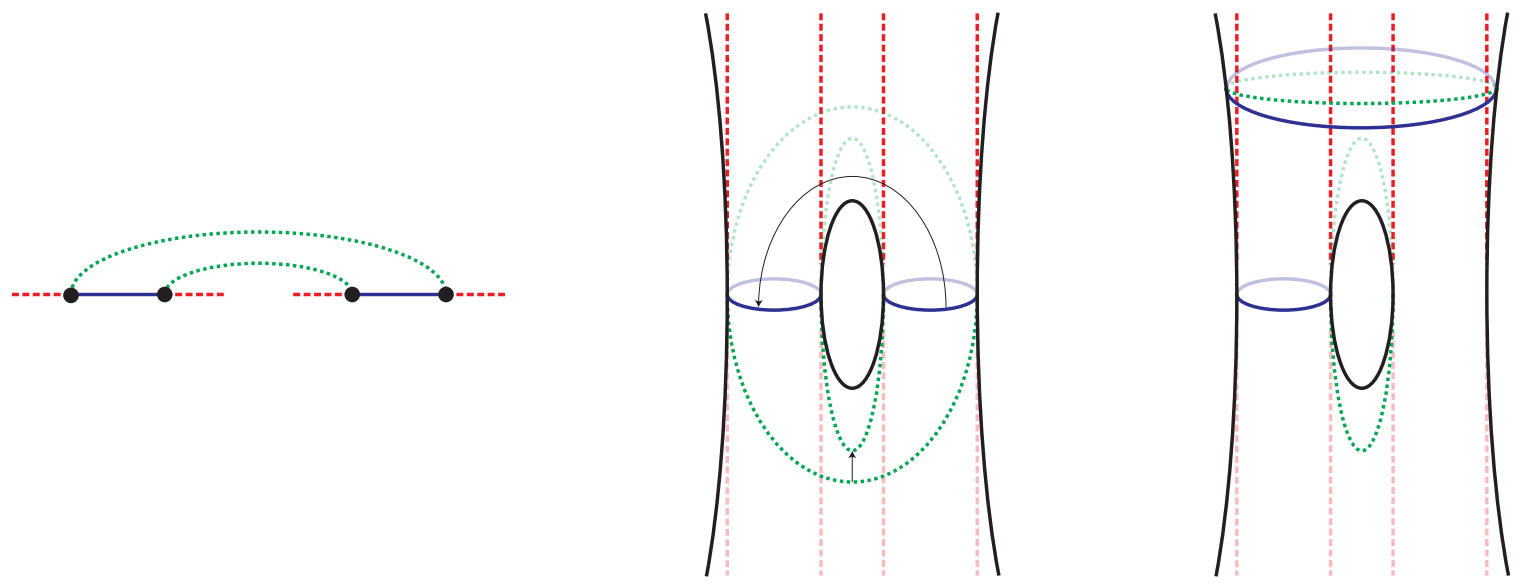

FIGURE 5. Strong equivalence to a subordinate diagram. Left: part of the bridge triple diagram where a saddle occurs. Center: the branched double cover of this diagram. Right: after performing handleslides according to the two small arrows in the center diagram, the resulting diagram is subordinate to a link.

$(\Sigma, \boldsymbol{\alpha}, \boldsymbol{\gamma})$ we may choose all of the complex structures to agree with a given $\mathbb{Z} / 2$-equivariant one, in which case the freed Floer complexes are just the ordinary Floer complexes tensored with the standard resolution for $\mathbb{F}_{2}$ over $\mathbb{F}_{2}[\mathbb{Z} / 2]$. Recall from Proposition 3.25 that to define the map of freed Floer complexes we choose a collection of families of perturbed almost complex structures over the triangle, as well as a collection of perturbations of $T_{\alpha}$. Consider the limit as these families of complex structures approach the constant family, and the perturbations become constant. By Gromov compactness, the holomorphic triangles converge to a holomorphic triangle with sequences of bigons at the three corners. Since the Lagrangians intersect only the discrete components of the fixed set, a generic $\mathbb{Z} / 2$-equivariant almost complex structure achieves transversality for all moduli spaces of non-constant triangles and bigons. Hence, the only limits with more than one component and Maslov index 0 must have a triangle with negative Maslov index contained in the fixed set. However, our choice of perturbations ensured that there are no negative-index holomorphic triangles contained in the fixed set. The index 0, constant holomorphic triangles are transversely cut out. (To see this, note that the constant triangles correspond to small, non-constant holomorphic triangles in a nearby, non-equivariant diagram; this uses the particular form of the perturbation in Figure 4.) It follows that counting index 0 holomorphic triangles with respect to a generic, $\mathbb{Z} / 2$-equivariant almost complex structure and unperturbed $T_{\alpha}$ gives the same map between freed Floer complexes as using a generic, nearby collection of perturbed almost complex structures and perturbations of $T_{\alpha}$. This map is induced by the count of index 0 holomorphic triangles between ordinary Floer complexes which agrees with the Ozsváth-Szabó cobordism map.

There is one last technical point for the saddle case. The cobordism map on $\widehat{H F}$, which in this case corresponds to a 2-handle attachment along a framed knot $C$, is defined using a Heegaard triple diagram subordinate to $C$. The Heegaard triple diagram $(\Sigma, \boldsymbol{\alpha}, \boldsymbol{\beta}, \boldsymbol{\gamma})$ is not subordinate to $C$, but it is strongly equivalent to a Heegaard triple subordinate to $C$ 

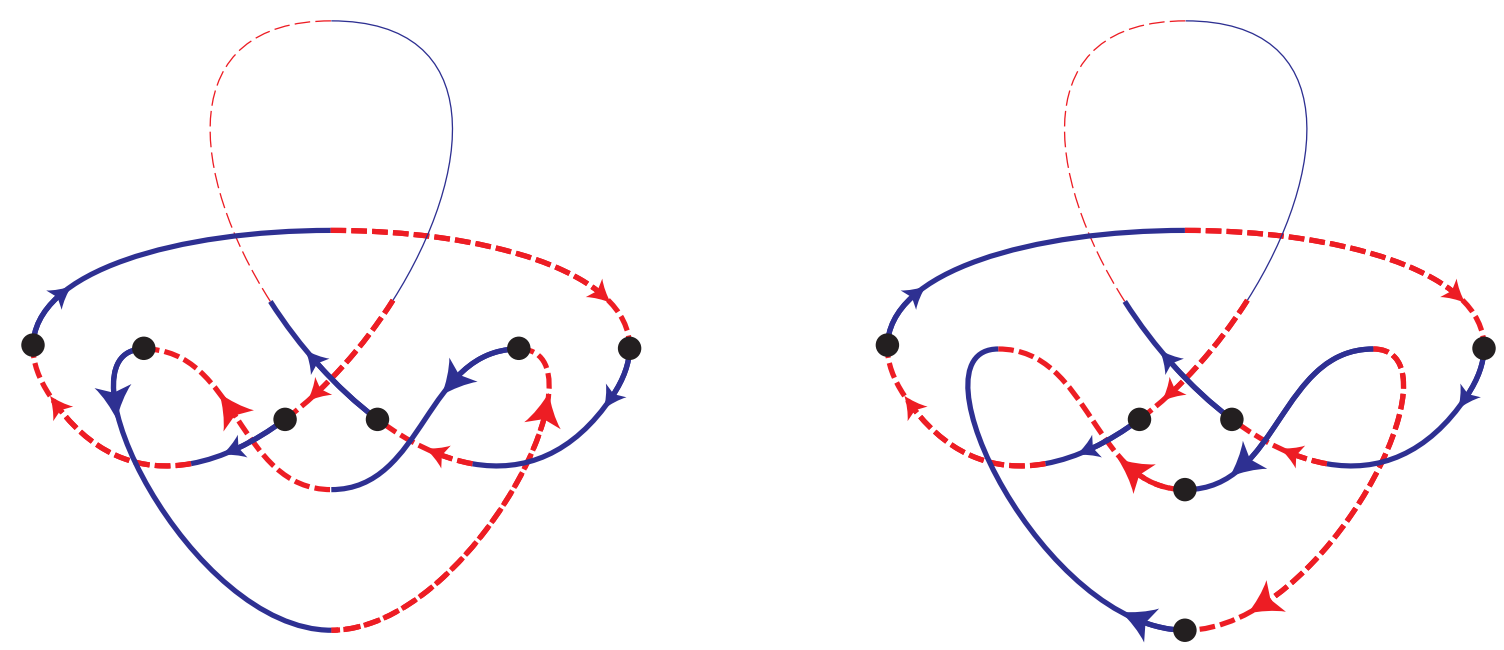

FIGURE 6. Fixed generators and orientations. The distinguished arcs on the based component are thin. Both choices of generators, corresponding to the two orientations of the unmarked component, are shown.

(see Figure 5), and so counting triangles in $(\Sigma, \boldsymbol{\alpha}, \boldsymbol{\beta}, \boldsymbol{\gamma})$ gives the Ozsváth-Szabó cobordism map on $\widehat{H F}$ [OSz06, Proof of Proposition 4.6]. This completes the argument for saddle cobordisms, and the proof.

Next we study the interaction of the cobordism maps with $\theta^{-1} \widehat{H F}_{\mathbb{Z} / 2}(\Sigma(L))$. Recall that $\theta^{-1} \widehat{H F}_{\mathbb{Z} / 2}(\Sigma(L))$ is identified with the Floer homology of the 0-dimensional Lagrangians $T_{\alpha}^{f i x}=T_{\alpha} \cap \operatorname{Fix}(\tau)$ and $T_{\beta}^{f i x}=T_{\beta} \cap \operatorname{Fix}(\tau)$, tensored with $\mathbb{F}_{2}\left[\theta, \theta^{-1}\right]$ (Proposition 6.3). To specify this identification, it helps to work with a particular class of diagrams. Call a diagram quite twisty if

- the fixed generators all lie in the same grading, and

- any non-fixed generator has grading strictly lower than any fixed generator.

Any bridge diagram can be made quite twisty by performing Reidemeister I moves at the endpoints of the arcs, which we will call twisting the diagram; see Figure 12. (A similar result in the context of symplectic Khovanov homology is Lemma 7.17; see that argument for some more details.) Figure 7 shows a quite twisty diagram for the unknot.

Given a quite twisty diagram $\left(\left\{A_{i}\right\},\left\{B_{i}\right\}\right)$ for $L$, the fixed generators span a quotient complex of $\widehat{C F}(\Sigma(L))$, and hence there is a chain inclusion $\widehat{C F}^{*}\left(T_{\alpha}^{f i x}, T_{\beta}^{f i x}\right) \rightarrow \widehat{C F}^{*}(\Sigma(L))$ (over $\mathbb{F}_{2}[\mathbb{Z} / 2]$ ). The induced map

$$
\theta^{-1} \widehat{H F}_{\mathbb{Z} / 2}\left(T_{\alpha}^{f i x}, T_{\beta}^{f i x}\right)=\widehat{H F}^{*}\left(T_{\alpha}^{f i x}, T_{\beta}^{f i x}\right) \otimes \mathbb{F}_{2}\left[\theta, \theta^{-1}\right] \rightarrow \theta^{-1} \widehat{H F}_{\mathbb{Z} / 2}(\Sigma(L))
$$

is an isomorphism; see Lemma 7.18. The Floer cohomology in the (discrete) fixed set is, of course, trivial, so each fixed generator gives a homology class in the localized equivariant Floer cohomology. The standard basis (of intersection points) for the Floer homology of the fixed set corresponds to the set of orientations of all of the unmarked components of $L$; see Figure 6. That is, if $L_{\bullet}$ is the marked component of $L$ then

$$
\theta^{-1} \widehat{H F}_{\mathbb{Z} / 2}(\Sigma(L)) \cong \mathbb{F}_{2}\left[\theta, \theta^{-1}\right]\left\langle\left\{\text { orientations of } L \backslash L_{\bullet}\right\}\right\rangle .
$$



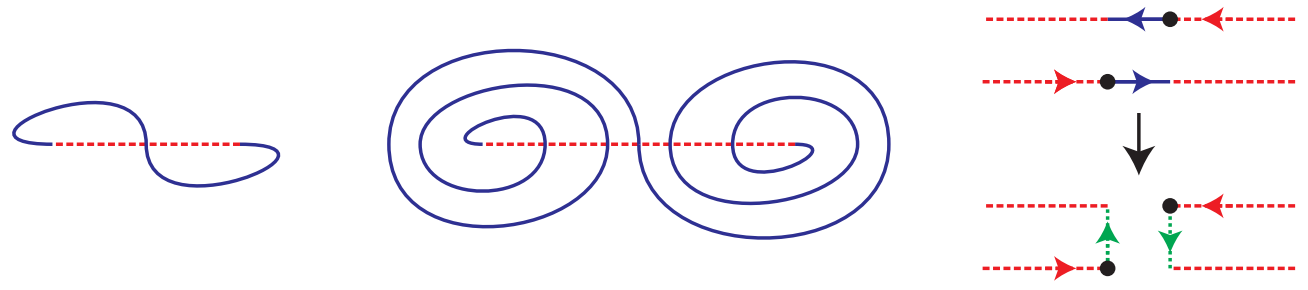

FiguRE 7. Orientations and cobordisms. Left: a quite twisty diagram for an unknot component. Center: an even more twisty unknot. Right: orientations and generators for an oriented saddle.

(Note that this is the same as the Lee homology of $L \backslash L$ • [Lee05, Theorem 4.2].) Since the two arcs on the marked component $L_{\bullet}$ are adjacent, $L_{\bullet}$ also inherits an orientation.

By a based cobordism between links $L_{0}$ and $L_{1}$ we mean a cobordism $T \subset[0,1] \times S^{3}$ so that the projection $\pi: T \rightarrow[0,1]$ is a Morse function, together with a section $\gamma:[0,1] \rightarrow T$ of $\pi$ so that $\operatorname{Im}(\gamma)$ is disjoint from $\operatorname{Crit}(\pi)$. In particular, every regular slice of a based cobordism is a based link.

Lemma 6.11. Given any connected, oriented, based cobordism $T$ between knots $K_{0}, K_{1} \subset S^{3}$, for suitable choices of auxiliary data the map

$$
\hat{f}_{\Sigma(T)}^{*}: \theta^{-1} \widehat{H F}_{\mathbb{Z} / 2}\left(\Sigma\left(K_{1}\right)\right) \rightarrow \theta^{-1} \widehat{H F}_{\mathbb{Z} / 2}\left(\Sigma\left(K_{0}\right)\right)
$$

induced by the map from Lemma 6.10 is an isomorphism.

(We will not show that $\hat{f}_{\Sigma(T)}$ or the induced map on equivariant cohomology is independent of the choices in its construction.)

Proof. We will prove the following:

(1) Given a diagram $L_{0}$ which has been twisted sufficiently and an isotopic link $L_{1}$, for some diagram of $L_{1}$ the Heegaard Floer continuation map $\widehat{C F}\left(\Sigma\left(L_{0}\right)\right) \rightarrow \widehat{C F}\left(\Sigma\left(L_{1}\right)\right)$ associated to the isotopy is such that the induced map

$\mathbb{F}_{2}\left[\theta, \theta^{-1}\right]\left\langle\left\{\right.\right.$ orientations of $\left.\left.L_{1} \backslash L_{1, \bullet}\right\}\right\rangle \cong \theta^{-1} \widehat{H F}_{\mathbb{Z} / 2}\left(\Sigma\left(L_{1}\right)\right)$

$$
\rightarrow \theta^{-1} \widehat{H F}_{\mathbb{Z} / 2}\left(\Sigma\left(L_{0}\right)\right) \cong \mathbb{F}_{2}\left[\theta, \theta^{-1}\right]\left\langle\left\{\text { orientations of } L_{0} \backslash L_{0, \bullet}\right\}\right\rangle
$$

agrees with the identification of sets of orientations induced by the isotopy. Further, by twisting $L_{0}$ the corresponding diagram $L_{1}$ can be made arbitrarily twisted.

(2) If $L_{1}$ is obtained from $L_{0}$ by a birth, $L_{0}$ is quite twisty, the new unknot component in $L_{1}$ is as on the left of Figure 7, and $o_{1}, o_{2}$ are the two orientations of the new unknot component of $L_{1}$, then the cobordism map

$$
\theta^{-1} \widehat{H F}_{\mathbb{Z} / 2}\left(\Sigma\left(L_{1}\right)\right) \cong \theta^{-1} \widehat{H F}_{\mathbb{Z} / 2}\left(\Sigma\left(L_{0}\right)\right) \otimes \mathbb{F}_{2}\left\langle o_{1}, o_{2}\right\rangle \rightarrow \theta^{-1} \widehat{H F}_{\mathbb{Z} / 2}\left(\Sigma\left(L_{0}\right)\right)
$$

from Lemma 6.10 is $x \otimes o_{1} \mapsto x, x \otimes o_{2} \mapsto x$. More generally, for $U$ as in the middle of Figure 7 the cobordism map is $x \otimes o_{1} \mapsto \theta^{-m} x, x \otimes o_{2} \mapsto \theta^{-m} x$ for some $m$.

(3) If $L_{1}$ is obtained from $L_{0}$ by a death, and $L_{0}$ and $L_{1}$ are quite twisty, and $o_{1}, o_{2}$ are the two orientations of the dying unknot component of $L_{0}$, then the cobordism map

$$
\theta^{-1} \widehat{H F}_{\mathbb{Z} / 2}\left(\Sigma\left(L_{1}\right)\right) \rightarrow \theta^{-1} \widehat{H F}_{\mathbb{Z} / 2}\left(\Sigma\left(L_{1}\right)\right) \otimes \mathbb{F}_{2}\left\langle o_{1}, o_{2}\right\rangle \cong \theta^{-1} \widehat{H F}_{\mathbb{Z} / 2}\left(\Sigma\left(L_{0}\right)\right)
$$


from Lemma 6.10 is $x \mapsto \theta^{-m} x \otimes\left(o_{1}+o_{2}\right)$ for some $m$.

(4) If $S$ is a saddle cobordism from $L_{0}$ to $L_{1}$ compatible with an orientation $o_{0}$ for $L_{0}$ and $o_{1}$ for $L_{1}$ then for quite twisty diagrams for $L_{0}$ and $L_{1}$ as in Figure 4, the induced $\operatorname{map} \theta^{-1} \widehat{H F}_{\mathbb{Z} / 2}\left(\Sigma\left(L_{1}\right)\right) \rightarrow \theta^{-1} \widehat{H F}_{\mathbb{Z} / 2}\left(\Sigma\left(L_{0}\right)\right)$ from Lemma 6.10 sends $o_{1}$ to $o_{0}$.

If we start with a diagram which has been twisted enough, and the births we use are also twisted enough, then we can arrange that all intermediate diagrams during the cobordism satisfy the conditions in Points (1)-(4) during the corresponding moves. The result then follows as in the case of Lee homology [Ras10, Corollary 4.2].

We start with Point (1), about bridge moves. For stabilizations near $p_{2 n}$, Point (1) is obvious. Next, consider an isotopy or handleslide; for concreteness, in the case of a handleslide assume it is of a $B$-arc over another $B$-arc. Choose a triple diagram $\left(\left\{A_{i}\right\},\left\{B_{i}\right\},\left\{C_{i}\right\}\right)$ for the isotopy or handleslide so that $\left(\left\{A_{i}\right\},\left\{C_{i}\right\}\right)$ and $\left(\left\{B_{i}\right\},\left\{C_{i}\right\}\right)$ are also quite twisty, the top-graded generator of $\widehat{H F}\left(T_{\beta}, T_{\gamma}\right)$ is in the same grading as the fixed generators $T_{\beta}^{f i x} \cap T_{\gamma}^{f i x}$, and there is a Maslov index zero triangle connecting some fixed generators $\mathbf{x}_{\alpha, \beta} \in T_{\alpha}^{f i x} \cap T_{\beta}^{f i x}$, $\mathbf{x}_{\beta, \gamma} \in T_{\beta}^{f i x} \cap T_{\gamma}^{f i x}$, and $\mathbf{x}_{\alpha, \gamma} \in T_{\alpha}^{f i x} \cap T_{\gamma}^{f i x}$. This can be arranged by twisting the diagram $\left(\left\{A_{i}\right\},\left\{B_{i}\right\}\right)$ sufficiently and then choosing $C_{i}$ to intersect the corresponding $B_{i}$ in one interior point (and the two endpoints). (In visualizing the handleslide, it may be helpful to visualize the $B$-arcs as straight and the $A$-arcs as twisty.)

Note that each of $T_{\alpha}^{f i x}, T_{\beta}^{f i x}$ and $T_{\gamma}^{f i x}$ consists of $2^{n-1}$ points, and by the hypothesis, the top-graded generator of $\widehat{C F}\left(T_{\beta}, T_{\gamma}\right)$ is $\mathbf{x}_{t o p}:=\sum_{\mathbf{x} \in T_{\beta}^{f i x} \cap T_{\gamma}^{f i x}} \mathbf{x}$.

Under the correspondences $\theta^{-1} \widehat{H F}_{\mathbb{Z} / 2}\left(\Sigma\left(L_{1}\right)\right) \cong \widehat{H F}^{*}\left(T_{\alpha}^{f i x}, T_{\gamma}^{f i x}\right)$ and $\theta^{-1} \widehat{H F}_{\mathbb{Z} / 2}\left(\Sigma\left(L_{0}\right)\right) \cong$ $\widehat{H F}^{*}\left(T_{\alpha}^{f i x}, T_{\beta}^{f i x}\right)$ between the localized equivariant Floer cohomology and the Floer cohomology in the fixed set, we claim that the map $\hat{f}_{\Sigma(T)}^{*}$ corresponds to the map which counts triangles in the fixed set. To see this, note that the triangle map is graded and, via small triangles, sends some fixed generators to fixed generators. Thus, the triangle map sends the grading containing the fixed generators to the grading containing the fixed generators, and hence fixed generators of $C F\left(T_{\alpha}, T_{\beta}\right)$ map only to fixed generators of $C F\left(T_{\alpha}, T_{\gamma}\right)$. Any triangles not entirely contained in the fixed set connecting fixed generators to fixed generators occur in pairs (via the $\mathbb{Z} / 2$-action), and hence do not contribute to the count with $\mathbb{F}_{2}$ coefficients. Thus, the map on fixed generators counts only triangles in the fixed set. It follows from the form of $\mathbf{x}_{t o p}$ that this map is the identity on $T_{\alpha}^{f i x} \cap T_{\beta}^{f i x}=T_{\alpha}^{f i x} \cap T_{\gamma}^{f i x}$. Tracing through the proof of Lemma 7.18 then proves the first statement.

The proof of the fourth statement, about saddle moves, is similar. We use the triple diagram from the proof of Lemma 6.10 (Figure 4), with the further requirement that both $\left(\left\{A_{i}\right\},\left\{B_{i}\right\}\right)$ and $\left(\left\{A_{i}\right\},\left\{C_{i}\right\}\right)$ are quite twisty; $\left\{B_{i}, C_{i}\right\}$ is quite twisty by construction. Again, the map $C F\left(T_{\alpha}^{f i x}, T_{\beta}^{f i x}\right) \rightarrow C F\left(T_{\alpha}^{f i x}, T_{\gamma}^{f i x}\right)$ on the fixed generators corresponds to counting triangles in the fixed set where the $\beta-\gamma$ corner maps to any point in $T_{\beta}^{f i x} \cap T_{\gamma}^{f i x}$. As illustrated in Figure 7, it follows that the map respects the identification with orientations.

Finally, we turn to births and deaths. First, for births, suppose that $L_{1}=L_{0} \amalg U$ where $U$ is the quite twisty diagram for the unknot shown on the left in Figure 7, and the disjoint union takes place in a region adjacent to the basepoint $p_{2 n}$. The map $\widehat{C F}\left(\Sigma\left(L_{0}\right)\right) \rightarrow \widehat{C F}\left(\Sigma\left(L_{1}\right)\right)$ sends $x$ to $x \otimes \Theta_{\text {top }}$. Note that $\Theta_{\text {top }}$ is the sum of the two fixed generators for $U$. Dualizing, the map $\widehat{C F}^{*}\left(\Sigma\left(L_{1}\right)\right) \rightarrow \widehat{C F}^{*}\left(\Sigma\left(L_{0}\right)\right)$ sends $x \otimes o_{i}$ to $x$. 
More generally, in order to ensure that later diagrams are quite twisty, we may need to create the unknot $U^{\prime}$ shown in the center of Figure 7, rather than the left. For this diagram, the complex $\widehat{C F}\left(\Sigma\left(U^{\prime}\right)\right)$ has the form:

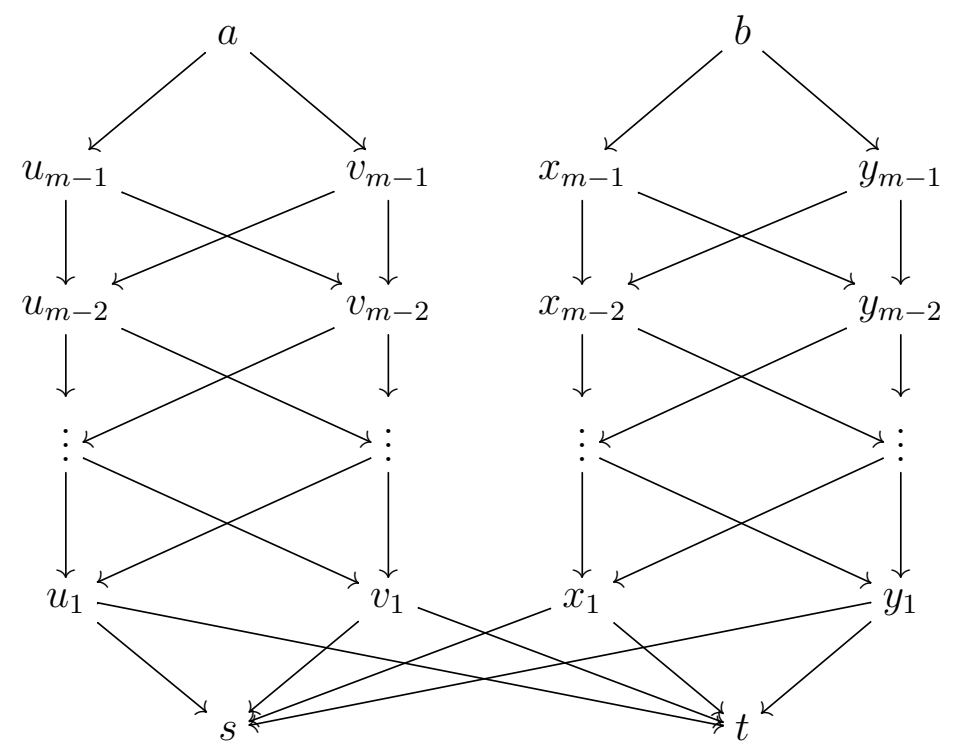

The map $\tau$ exchanges $u_{i} \leftrightarrow v_{i}, x_{i} \leftrightarrow y_{i}$, and $s \leftrightarrow t$. The fixed generators are the top two, $a$ and $b$, but the homology is supported in the bottom two gradings.

We are interested in the composition of the birth map with the twisting isotopy from the slightly twisty unknot $U$ to the arbitrarily twisty unknot $U^{\prime}$. Call this composition $\phi$ : $\widehat{C F}_{\mathbb{Z} / 2}\left(\Sigma\left(L_{1}\right)\right)=\widehat{C F}_{\mathbb{Z} / 2}\left(\Sigma\left(L_{0}\right)\right) \otimes_{\mathbb{F}_{2}[\theta]} \widehat{C F}_{\mathbb{Z} / 2}\left(\Sigma\left(U^{\prime}\right)\right) \rightarrow \widehat{C F}_{\mathbb{Z} / 2}\left(\Sigma\left(L_{0}\right)\right)$. (Here, we are using the standard equivariant cochain complex, Formula (2.2).) Since $U^{\prime}$ is adjacent to the basepoint, $\phi$ must be induced by a projection map $\pi: \widehat{C F}_{\mathbb{Z} / 2}\left(\Sigma\left(U^{\prime}\right)\right) \rightarrow \mathbb{F}_{2}\left[\theta, \theta^{-1}\right]$. Since on the $E^{1}$-page of the spectral sequence associated to the $\theta$-power filtration, $\pi$ agrees with the dual of the Heegaard Floer cobordism map (see Lemma 6.10), $\pi$ must send $x \otimes\left(u_{1}^{*}+v_{1}^{*}\right)$ and $x \otimes\left(x_{1}^{*}+y_{1}^{*}\right)$ to $x$. In the localized equivariant complex, there are homologies

$$
\begin{aligned}
u_{i}^{*}+v_{i}^{*} & \sim \theta^{-1}\left(u_{i+1}^{*}+v_{i+1}^{*}\right) & x_{i}^{*}+y_{i}^{*} & \sim \theta^{-1}\left(x_{i+1}^{*}+y_{i+1}^{*}\right) \\
u_{m-1}^{*}+v_{m-1}^{*} & \sim \theta^{-1} a^{*} & x_{m-1}^{*}+y_{m-1}^{*} & \sim \theta^{-1} b^{*} .
\end{aligned}
$$

The generators $a^{*}$ and $b^{*}$ represent the two orientations, so the birth map must send $x \otimes o_{i}$ to $\theta^{1-m} x$, as desired.

Deaths are similar. Suppose $L_{0}=L_{1} \amalg U$ where $U$ is a quite twisty diagram for the unknot. Again, we may assume that $U$ has the form shown in the middle of Figure 7, and is adjacent to the basepoint $p_{2 n}$. The Ozsváth-Szabó map $\widehat{C F}\left(\Sigma\left(L_{0}\right)\right) \rightarrow \widehat{C F}\left(\Sigma\left(L_{1}\right)\right)$ sends $x \otimes \Theta_{\text {bot }}$ to $x$ and other generators to 0 . Thus, the cobordism map $\widehat{C F}_{\mathbb{Z} / 2}\left(\Sigma\left(L_{1}\right)\right) \rightarrow \widehat{C F}_{\mathbb{Z} / 2}\left(\Sigma\left(L_{0}\right)\right) \cong$ $\widehat{C F}_{\mathbb{Z} / 2}\left(\Sigma\left(L_{1}\right)\right) \otimes_{\mathbb{F}_{2}[\theta]} \widehat{C F}_{\mathbb{Z} / 2}\left(\Sigma\left(U^{\prime}\right)\right)$ must send $x$ to $x \otimes\left(s^{*}+t^{*}\right)$. In the localized equivariant complex, $s^{*}+t^{*}$ is homologous to $\theta^{-1}\left(u_{1}^{*}+v_{1}^{*}+x_{1}^{*}+y_{1}^{*}\right)$ and hence to $\theta^{-m}\left(a^{*}+b^{*}\right)$, so the map sends $x$ to $x \otimes \theta^{-m}\left(a^{*}+b^{*}\right)=\theta^{-m} x \otimes\left(o_{1}+o_{2}\right)$, as desired.

Proof of Proposition 6.9. With Lemmas 6.10 and 6.11, the proof follows the same basic structure as the proof that $s$ is a concordance invariant [Ras10, Theorem 1]. Recall that

$$
q_{\tau}(K)=2 \min \left\{\operatorname{gr}(x) \mid x \in \widehat{H F}_{\mathbb{Z} / 2}(\Sigma(K)) / \text { tors }\right\},
$$



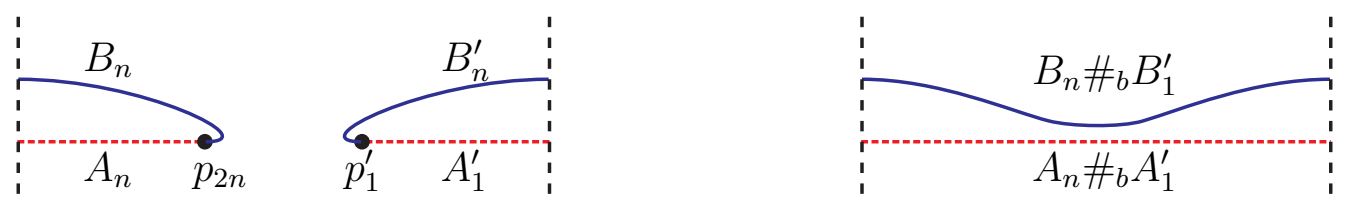

Figure 8. A bridge diagram for the connected sum.

where tors denotes the subspace annihilated by $\theta^{m}$ for some $m$. (Note that tors is the sum of its homogeneous pieces, since the equation $\theta^{m} x=d(y)$ implies that each graded piece of $x$ is also a boundary, so $\widehat{H F}_{\mathbb{Z} / 2}(\Sigma(K)) /$ tors inherits a grading from $\widehat{H F}_{\mathbb{Z} / 2}(\Sigma(K))$.)

Suppose that $T$ is a concordance between knots $K_{0}$ and $K_{1}$. By Lemma 6.10, there is a corresponding map $g_{\Sigma(T)}^{\mathbb{Z} / 2}: \widehat{H F}_{\mathbb{Z} / 2}\left(\Sigma\left(K_{1}\right)\right) \rightarrow \widehat{H F}_{\mathbb{Z} / 2}\left(\Sigma\left(K_{0}\right)\right)$ which by Lemma 6.11 induces an injective map

$$
\mathbb{F}_{2}[\theta] \cong \widehat{H F}_{\mathbb{Z} / 2}\left(\Sigma\left(K_{1}\right)\right) / \text { tors } \rightarrow \widehat{H F}_{\mathbb{Z} / 2}\left(\Sigma\left(K_{0}\right)\right) / \text { tors } \cong \mathbb{F}_{2}[\theta] .
$$

Since $\Sigma(T)$ is a rational homology cobordism between rational homology spheres, the map $g_{\Sigma(T)}$ preserves the absolute Maslov grading. Now, if $x$ is a non-zero element of the module $\widehat{H F}_{\mathbb{Z} / 2}\left(\Sigma\left(K_{1}\right)\right) /$ tors in minimal grading then its image $g_{\Sigma(T)}^{\mathbb{Z} / 2}(x)$ is a non-zero element of $\widehat{H F}_{\mathbb{Z} / 2}\left(\Sigma\left(K_{0}\right)\right) /$ tors, and so:

$$
q_{\tau}\left(K_{1}\right)=2 \operatorname{gr}(x)=2 \operatorname{gr}\left(g_{\Sigma(T)}(x)\right) \geq q_{\tau}\left(K_{0}\right) .
$$

Applying the same argument with the roles of $K_{0}$ and $K_{1}$ exchanged gives the opposite inequality, so $q_{\tau}\left(K_{0}\right)=q_{\tau}\left(K_{1}\right)$, as desired.

Proposition 6.12. The number $q_{\tau}$ satisfies $q_{\tau}\left(K_{1} \# K_{2}\right)=q_{\tau}\left(K_{1}\right)+q_{\tau}\left(K_{2}\right)$ and $q_{\tau}(m(K))=$ $-q_{\tau}(K)$.

Proof. We start with connected sums. Fix bridge diagrams $\left(\left\{A_{i}\right\},\left\{B_{i}\right\}\right)$ and $\left(\left\{A_{i}^{\prime}\right\},\left\{B_{i}^{\prime}\right\}\right)$ for $K_{1}$ and $K_{2}$, respectively. Let $A_{n}$ and $B_{n}$ (respectively $A_{1}^{\prime}$ and $B_{1}^{\prime}$ ) be the arcs incident to a marked point $p_{2 n}$ (respectively $p_{1}^{\prime}$ ). Taking the connected sum of the bridge diagrams at $p_{2 n}$ and $p_{1}^{\prime}$ gives a bridge diagram for $K_{1} \# K_{2}$, with $\operatorname{arcs} A_{1}, \ldots, A_{n-1}, A_{n} \#_{b} A_{1}^{\prime}, A_{2}^{\prime}, \ldots, A_{m}^{\prime}$ and $B_{1}, \ldots, B_{n-1}, B_{n} \#_{b} B_{1}^{\prime}, B_{2}^{\prime}, \ldots, B_{m}^{\prime}$. (See Figure 8; $A_{n} \#_{b} A_{1}^{\prime}$ and $B_{n} \#_{b} B_{1}^{\prime}$ are boundary connected sums with respect to boundary points $p_{2 n}$ and $p_{1}^{\prime}$.) If we choose $A_{n}$ and $B_{n}$ as the deleted arcs for $K_{1}, A_{1}^{\prime}$ and $B_{1}^{\prime}$ as the deleted arcs for $K_{2}$, and $A_{n} \#_{b} A_{1}^{\prime}$ and $B_{n} \#_{b} B_{1}^{\prime}$ as the deleted arcs for $K_{1} \# K_{2}$ then for appropriate choices of almost complex structures there is an isomorphism of complexes over $\mathbb{F}_{2}[\mathbb{Z} / 2]$

$$
\widehat{C F}\left(\Sigma\left(K_{1}\right)\right) \otimes_{\mathbb{F}_{2}} \widehat{C F}\left(\Sigma\left(K_{2}\right)\right) \cong \widehat{C F}\left(\Sigma\left(K_{1} \# K_{2}\right)\right)
$$

with the diagonal action of $\mathbb{Z} / 2$ on the left hand side. Further, this isomorphism respects the absolute $\mathbb{Q}$-grading (an immediate consequence of [OSz03a, Theorem 4.3]).

Thus, $\widehat{C F}_{\mathbb{Z} / 2}\left(\Sigma\left(K_{1} \# K_{2}\right)\right)$ is isomorphic to $\widehat{C F}_{\mathbb{Z} / 2}\left(\Sigma\left(K_{1}\right)\right) \otimes_{\mathbb{F}_{2}[\theta]} \widehat{C F}_{\mathbb{Z} / 2}\left(\Sigma\left(K_{2}\right)\right)$, but the differentials are superficially different: the differential on $\widehat{C F}_{\mathbb{Z} / 2}\left(\Sigma\left(K_{1} \# K_{2}\right)\right)$ is $d\left(\theta^{i} x \otimes y\right)=$ $\theta^{i}\left[d_{\widehat{C F}}(x) \otimes y+x \otimes d_{\widehat{C F}}(y)\right]+\theta^{i+1}[x \otimes y+\tau(x) \otimes \tau(y)]$, while the tensor product differential is $d\left(\theta^{i} x \otimes y\right)=\theta^{i}\left[d_{\widehat{C F}}(x) \otimes y+x \otimes d_{\widehat{C F}}(y)\right]+\theta^{i+1}[\tau(x) \otimes y+x \otimes \tau(y)]$. The map 
$\phi: \widehat{C F}_{\mathbb{Z} / 2}\left(\Sigma\left(K_{1}\right)\right) \otimes_{\mathbb{F}_{2}[\theta]} \widehat{C F}_{\mathbb{Z} / 2}\left(\Sigma\left(K_{2}\right)\right) \rightarrow \widehat{C F}_{\mathbb{Z} / 2}\left(\Sigma\left(K_{1} \# K_{2}\right)\right), \phi\left(\theta^{i} x \otimes y\right)=\theta^{i} x \otimes \tau^{i}(y)$ intertwines the two differentials. This map does not respect the $\mathbb{F}_{2}[\theta]$-module structures, but does respect the action of $\mathbb{F}_{2}\left[\theta^{2}\right]$.

By the universal coefficient theorem, $H_{*}\left(\widehat{C F}_{\mathbb{Z} / 2}\left(\Sigma\left(K_{1}\right)\right) \otimes_{\mathbb{F}_{2}[\theta]} \widehat{C F}_{\mathbb{Z} / 2}\left(\Sigma\left(K_{2}\right)\right)\right) /$ tors is isomorphic to $\widehat{H F}_{\mathbb{Z} / 2}\left(\Sigma\left(K_{1}\right)\right) /$ tors $\otimes \widehat{H F}_{\mathbb{Z} / 2}\left(\Sigma\left(K_{2}\right)\right) /$ tors. (Here, tors stands for the submodule annihilated by $\theta^{m}$ for some $m$.) Since the map $\phi$ respects the action of $\mathbb{F}_{2}\left[\theta^{2}\right]$, it also takes (non-)torsion classes to (non-)torsion classes. Thus, the composition

$$
\begin{aligned}
& \widehat{H F}_{\mathbb{Z} / 2}\left(\Sigma\left(K_{1}\right)\right) / \text { tors } \otimes \widehat{H F}_{\mathbb{Z} / 2}\left(\Sigma\left(K_{2}\right)\right) / \text { tors } \longrightarrow H_{*}\left(\widehat{C F}_{\mathbb{Z} / 2}\left(\Sigma\left(K_{1}\right)\right) \otimes_{\mathbb{F}_{2}[\theta]} \widehat{C F}_{\mathbb{Z} / 2}\left(\Sigma\left(K_{2}\right)\right)\right) / \text { tors } \\
& \stackrel{\phi}{\longrightarrow} \widehat{H F}_{\mathbb{Z} / 2}\left(\Sigma\left(K_{1} \# K_{2}\right)\right) / \text { tors }
\end{aligned}
$$

is an isomorphism. It follows that $q_{\tau}\left(K_{1} \# K_{2}\right)=q_{\tau}\left(K_{1}\right)+q_{\tau}\left(K_{2}\right)$.

The argument for mirrors is easier. Mirroring a knot corresponds to exchanging the $A_{i}$ and $B_{i}$ arcs, which has the effect of replacing $\widehat{C F}(\Sigma(K))$ with $\operatorname{Hom}_{\mathbb{F}_{2}}\left(\widehat{C F}(\Sigma(K)), \mathbb{F}_{2}\right)$. The behavior on gradings is that if $\left\{x_{i}\right\}$ is a homogeneous basis for $\widehat{C F}(\Sigma(K))$ with dual basis $\left\{x_{i}^{*}\right\}$ then the grading of $x_{i}^{*}$, viewed as an element in $\widehat{C F}(\Sigma(m(K)))$, is minus the grading of $x_{i}$ [OSz03a, Proposition 4.2]. This isomorphism holds $\mathbb{Z} / 2$-equivariantly. It follows that $\widehat{C F}_{\mathbb{Z} / 2}(\Sigma(m(K))) \cong \operatorname{Hom}_{\mathbb{F}_{2}[\theta]}\left(\widehat{C F}_{\mathbb{Z} / 2}(\Sigma(K)), \mathbb{F}_{2}[\theta]\right)$. Applying the universal coefficient theorem,

$$
\widehat{H F}_{\mathbb{Z} / 2}(\Sigma(m(K))) / \text { tors } \cong \operatorname{Hom}_{\mathbb{F}_{2}[\theta]}\left(\widehat{H F}_{\mathbb{Z} / 2}(\Sigma(K)) / \text { tors, } \mathbb{F}_{2}[\theta]\right),
$$

and it follows that $q_{\tau}(m(K))=-q_{\tau}(K)$.

Proof of Theorem 1.25. This is simply a restatement of Propositions 6.9 and 6.12.

6.2. Many concordance invariants from the $\mathbb{Z} / 2$-action on $C F^{-}(\Sigma(K))$. For this section, for convenience, we will restrict our attention to the case that $K$ is a knot, not a link.

Consider the freed complex $\widetilde{C F}^{-}(\Sigma(K))$ for the Heegaard Floer invariant $H F^{-}(\Sigma(K))$. Unlike $\widehat{C F}(\Sigma(K))$, the complex $C F^{-}(\Sigma(K))$ counts curves in the closed symplectic manifold $\operatorname{Sym}^{g}(\Sigma)$, which does not satisfy Item (J-1) of Hypothesis 3.2. The construction of the freed Floer complex used this part of the hypothesis for two reasons: for energy bounds allowing us to work with $\mathbb{F}_{2}$ (or $\mathbb{F}_{2}[U]$ ) coefficients instead of Novikov coefficients, and for a relative $\mathbb{Z}$-grading. To work with $\mathbb{F}_{2}$ coefficients, the following weaker condition suffices:

$\left(\mathrm{J}^{\prime}-1\right)$ There is a non-negative $\tau \in \mathbb{R}$ so that for any loop of paths

$$
v:\left([0,1] \times S^{1},\{0\} \times S^{1},\{1\} \times S^{1}\right) \rightarrow\left(M, L_{0}, L_{1}\right)
$$

such that each $\left[\left.v\right|_{[0,1] \times\{p t\}}\right]$ is homotopic to a constant path from $L_{0}$ to $L_{1}$, the area of $v$ (with respect to $\omega$ ) is $\tau$ times the Maslov index of $v$. (Again, compare, for example, [WW12, Theorem 1.0.1].)

For $C F^{-}(\Sigma(K))$, one actually works with coefficients in $\mathbb{F}_{2}[U]$, where the power of $U$ keeps track of the intersection number with the divisor $V_{z}$ in the symmetric product corresponding to some basepoint $z$ on the Heegaard surface. The resulting complex is a relatively $\mathbb{Z}_{\text {- }}$ graded complex over $\mathbb{F}_{2}[U]$, where $U$ has degree -2 [OSz04b, Section 4.2]. With these observations, the freed Floer complex is defined as in Section 3.4, with $U$-powers always tracking intersections numbers with $V_{z}$. The equivariant Floer complex is

$$
C F_{\mathbb{Z} / 2}^{-}(\Sigma(K))=\operatorname{Hom}_{\mathbb{F}_{2}[U][\mathbb{Z} / 2]}\left(\widetilde{C F}^{-}(\Sigma(K)), \mathbb{F}_{2}[U]\right)
$$


and the equivariant Floer cohomology $H F_{\mathbb{Z} / 2}^{-}(\Sigma(K))$ is the homology of $C F_{\mathbb{Z} / 2}^{-}$. Both the equivariant complex $\mathrm{CF}_{\mathbb{Z} / 2}^{-}(\Sigma(K))$ and its homology $\mathrm{HF}_{\mathbb{Z} / 2}^{-}(\Sigma(K))$ are modules over $\mathbb{F}_{2}[U, \theta]$. As discussed earlier in the case of $\widehat{C F}$, there are no non-constant holomorphic disks contained in the fixed set, so by Proposition 4.3 we can compute $H F_{\mathbb{Z} / 2}^{-}(\Sigma(K))$ using a generic 1parameter family of $\mathbb{Z} / 2$-equivariant almost complex structures.

In what follows, we will be particularly interested in the summand $H^{-}\left(\Sigma(K), \mathfrak{s}_{0}\right)$, where $\mathfrak{s}_{0}$ is the unique spin structure on $\Sigma(K)$, also called the central spin $^{c}$-structure. The involution $\tau: \Sigma(K) \rightarrow \Sigma(K)$ acts on $\operatorname{Spin}^{c}(Y)$ by conjugation and fixes only $\mathfrak{s}_{0}$ [Gri06, page 1378], [Lev08, Remark 3.4]. Therefore, $C F_{\mathbb{Z} / 2}^{-}(\Sigma(K))$ splits along orbits of $\operatorname{spin}^{c}$-structures.

Theorem 6.13. There are isomorphisms between the completed, localized equivariant Floer homologies

$$
\begin{aligned}
U^{-1} H F_{\mathbb{Z} / 2}^{-}(\Sigma(K)) \otimes_{\mathbb{F}_{2}[\theta]} \mathbb{F}_{2}[[\theta]] & \cong \mathbb{F}_{2}\left[U, U^{-1}\right][[\theta]] \\
\theta^{-1} H F_{\mathbb{Z} / 2}^{-}(\Sigma(K)) \otimes_{\mathbb{F}_{2}[U]} \mathbb{F}_{2}[[U]] & \cong \mathbb{F}_{2}\left[\theta, \theta^{-1}\right][[U]] \\
(U \theta)^{-1} H F_{\mathbb{Z} / 2}^{-}(\Sigma(K)) \otimes_{\mathbb{F}_{2}[U, \theta]} \mathbb{F}_{2}[[U, \theta]] & \cong \mathbb{F}_{2}[[U, \theta]]\left[U^{-1}, \theta^{-1}\right] .
\end{aligned}
$$

Proof. We start with the proof of Equation (6.14). Consider the filtration of $C F_{\mathbb{Z} / 2}^{-}\left(T_{\alpha}, T_{\beta}\right)$ by the $\theta$-power, i.e., so that, using the notation of Observation 3.16, $\alpha_{n}^{*} \otimes x^{*}$ lies in filtration $n$. The $E_{1}$-page of the associated spectral sequence is isomorphic to $H F^{-, *}(\Sigma(K)) \otimes \mathbb{F}_{2}[\theta]$. Each $H F^{-, *}(\Sigma(K)) \otimes\left\{\theta^{i}\right\}$ decomposes (non-canonically) as $\mathbb{F}_{2}[U] \oplus H F^{r e d, *}(\Sigma(K)$ ) where $H F^{r e d, *}(\Sigma(K))$ is $U^{N}$-torsion for some $N$ [OSz04a, Theorem 10.1], so the $E_{1}$-page itself is $\mathbb{F}_{2}[U, \theta] \oplus H F^{r e d, *}(\Sigma(K)) \otimes \mathbb{F}_{2}[\theta]$. Let $x$ be a generator of the $\mathbb{F}_{2}[U, \theta]$ submodule. It follows from the fact that the induced differentials $d_{r}$ on the $r$ th page of the spectral sequence commute with the $\mathbb{F}_{2}[U, \theta]$-module structure and $\left(d_{r}\right)^{2}=0$ that $d_{r}\left(U^{n} x\right)$ must be $U$-torsion. It then follows from finite-dimensionality of $H F^{\text {red,* }}(\Sigma(K))$ and the $\mathbb{F}_{2}[U, \theta]$-module structure that $d_{r}=0$ for $r$ sufficiently large. Thus, the $E_{\infty}$-page of the spectral sequence is isomorphic to $\mathbb{F}_{2}[U, \theta]\left\langle U^{n} x\right\rangle \oplus T$, where $T$ is $U^{N}$-torsion (for some $N$ ).

Now, choose a cycle $x_{n} \in C F_{\mathbb{Z} / 2}^{-}\left(T_{\alpha}, T_{\beta}\right)$ whose image in the associated graded complex $E_{0}$ represents $U^{n} x$. There is a corresponding map $\mathbb{F}_{2}[U, \theta] \rightarrow C F_{\mathbb{Z} / 2}^{-}\left(T_{\alpha}, T_{\beta}\right)$ given by $\left(U^{i} \theta^{j}\right) \mapsto$ $U^{i} \theta^{j} x_{n}$. The induced map $\mathbb{F}_{2}\left[U, U^{-1}, \theta\right] \rightarrow U^{-1} C F_{\mathbb{Z} / 2}^{-}\left(T_{\alpha}, T_{\beta}\right)$ is by definition a map of $\mathbb{F}_{2}\left[U, U^{-1}, \theta\right]$-modules. If we endow $\mathbb{F}_{2}\left[U, U^{-1}, \theta\right]$ with the filtration $\mathcal{F}\left(U^{i} \theta^{j}\right)=j$ then the map respects the filtrations and induces an isomorphism on the $E_{1}$-page of the spectral sequence, and hence after completing with respect to $\theta$ the map itself is a filtered quasiisomorphism [McC01, Corollary 3.15].

The proof of the Equation (6.15) is the same as the proof of Equation (6.14), with the roles of $U$ and $\theta$ exchanged, and using Proposition 6.3 in place of Ozsváth-Szabó's computation of $H F^{\infty}$ [OSz04a, Theorem 10.1] to analyze the $E_{1}$-page of the spectral sequence. Equation (6.16) is immediate from either of Equation (6.14) or (6.15).

Before we discuss how to extract numerical invariants from $C F_{\mathbb{Z} / 2}^{-}\left(\Sigma(K), \mathfrak{s}_{0}\right)$, we pause for a remark about dualizing the chain complex $C F^{-}\left(\Sigma(K), \mathfrak{s}_{0}\right)$.

Remark 6.17. Below, we will often use the Heegaard Floer cohomology $H F^{-, *}\left(\Sigma(K), \mathfrak{s}_{0}\right)$, which we take to be the homology of the dual complex $\operatorname{Hom}_{\mathbb{F}_{2}[U]}\left(C F^{-}\left(\Sigma(K), \mathfrak{s}_{0}\right), \mathbb{F}_{2}[U]\right)$. This is a different Floer cohomology from Ozsváth-Szabó [OSz04a, Section 2.2]: we have 


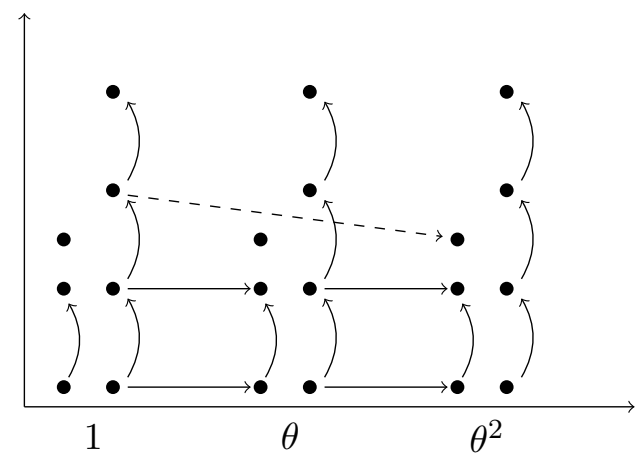

Figure 9. A model for $H F^{-}$spectral sequence. This is a sample $E_{1^{-}}$ page for the spectral sequence associated to the filtration of $H F_{\mathbb{Z} / 2}\left(\Sigma(K), \mathfrak{s}_{0}\right)$ by the $\theta$-power. In each filtration level we have a copy of $H F^{-, *}\left(\Sigma(K), \mathfrak{s}_{0}\right)$; the vertical axis is the homological grading in this complex. Curved vertical arrows denote the action of $U$; horizontal arrows show a possible nontrivial $d_{1}$ differential. The dashed arrow is a possible $d_{2}$ differential on the next page of the spectral sequence.

dualized over $\mathbb{F}_{2}[U]$ rather than over $\mathbb{F}_{2}$. Consequently, $H F^{-, *}\left(\Sigma(K), \mathfrak{s}_{0}\right)$ is isomorphic to $H F^{-}\left(-\Sigma(K), \mathfrak{s}_{0}\right)$. If this isomorphism sends $x$ to $x^{\prime}$, then $\operatorname{gr}(x)=-\operatorname{gr}\left(x^{\prime}\right)-4$.

We can now discuss how to extract numerical invariants from $C F_{\mathbb{Z} / 2}^{-}\left(\Sigma(K), \mathfrak{s}_{0}\right)$. Consider the filtration of $C_{\mathbb{Z} / 2}^{-}\left(\Sigma(K), \mathfrak{s}_{0}\right)$ by the $\theta$-power. The $E_{0}$-page of the associated spectral sequence is, in each filtration level, a copy of the dual complex $\operatorname{Hom}_{\mathbb{F}_{2}[U]}\left(C F^{-}\left(\Sigma(K), \mathfrak{s}_{0}\right), \mathbb{F}_{2}[U]\right)$. The homology of this complex is the Heegaard Floer cohomology $H F^{-, *}\left(\Sigma(K), \mathfrak{s}_{0}\right)$, so the $E_{1}$-page of the spectral sequence is $H F^{-, *}\left(\Sigma(K), \mathfrak{s}_{0}\right) \otimes \mathbb{F}_{2}[\theta]$. Let $\operatorname{gr}(x)$ denote the cohomological grading on the spectral sequence, so the differential raises gr by 1 . With respect to the grading gr, the variables $U$ and $\theta$ have degrees 2 and 1 respectively.

As an $\mathbb{F}_{2}[U]$ module, $H F^{-, *}\left(\Sigma(K), \mathfrak{s}_{0}\right)$ decomposes into the direct sum of two submodules. One is a canonical finite dimensional $\mathbb{F}_{2}$-vector space $R_{1}=H F^{r e d, *}\left(\Sigma(K), \mathfrak{s}_{0}\right)$ consisting of elements which are $U^{n}$ torsion for some $n$. The other is a (non-canonical) copy of $\mathbb{F}_{2}[U]$. The minimal grading of a non- $U$-torsion homogeneous element of $H F^{-, *}\left(\Sigma(K), \mathfrak{s}_{0}\right)$ is an invariant of the pair $\left(\Sigma(K), \mathfrak{s}_{0}\right)$ which is equal to the Ozsváth-Szabó correction term $d\left(\Sigma(K), \mathfrak{s}_{0}\right)$ minus two [OSz03a, Section 4]. Let $x$ be a homogeneous generator of the $\mathbb{F}_{2}[U]$ submodule. Since the first page of the spectral sequence associated to the $\theta$-filtration of $C F_{\mathbb{Z} / 2}^{-}\left(\Sigma(K)\right.$, $\left.\mathfrak{s}_{0}\right)$ is equal to $H F^{-, *}\left(\Sigma(K), \mathfrak{s}_{0}\right) \otimes \mathbb{F}_{2}[\theta]$, it must decompose as $\mathbb{F}_{2}[U, \theta] \oplus\left(R_{1} \otimes \mathbb{F}_{2}[\theta]\right)$, where $\mathbb{F}_{2}[U, \theta]$ is generated by $x$.

Now consider the differential $d_{1}$ on this complex, which raises the $\theta$-power by one. This differential is equivariant with respect to the actions of both $U$ and $\theta$. Indeed, because $\left(d_{1}\right)^{2}=0$, we see that $d_{1}$ is identically zero on $U^{n} E_{1} \cong \mathbb{F}_{2}[U, \theta]$ for sufficiently high $n$. Let $d_{1}(x)=\theta y ; y$ is forced to be $U$-torsion, so let $m$ be the smallest integer so that $U^{m} y=0$. The $E_{2}$-page of the spectral sequence is given by $\mathbb{F}_{2}[U, \theta] \oplus R_{2}$, where $R_{2}$ is a vector space consisting of elements that are $U^{n}$ torsion for some $n$ (and may or may not be $\theta$-torsion) and $\mathbb{F}_{2}[U, \theta]$ is generated by $\left[U^{m} x\right]$. A model of such a spectral sequence is drawn in Figure 9.

We see a similar story on subsequent pages: on page $E_{s}$, the differential $d_{s}$ is trivial on $U^{n} E_{s}$ for $n \gg 0$, but there may be nontrivial differentials from elements which are not 
$U^{n}$-torsion to elements that lie in low $U$-gradings. So, define

$$
d_{\tau}(K, s)=\min \left\{i \mid \exists x \in E_{s}, \operatorname{gr}(x)=i, U^{n} x \neq 0 \forall n\right\}+2 .
$$

Notice that $d_{\tau}(K, 1)=d\left(\Sigma(K), \mathfrak{s}_{0}\right)$, which is, up to scaling, the concordance homomorphism $\delta$ studied by Manolescu-Owens [MO07]: $d_{\tau}(K, 1)=\delta(K) / 2$. Also, $d_{\tau}(K, s) \leq d_{\tau}(K, t)$ whenever $s<t$, and finiteness of the vector space $R_{1}$ implies that $\left\{d_{\tau}(K, s)\right\}$ assumes at most finitely many values over all natural numbers $s$.

Theorem 6.18. The quasi-isomorphism class of $\mathrm{CF}_{\mathbb{Z} / 2}^{-}(\Sigma(K))$, as a gr-graded chain complex over $\mathbb{F}_{2}[U, \theta]$, is an invariant of the knot $K$.

Proof. The proof is the same as the proofs of Theorem 1.24 and Proposition 6.7, with two caveats. First, for the surgery exact triangle to hold for $\mathrm{HF}^{-}$, we must restrict to the torsion spin ${ }^{c}$-structure on the new $S^{2} \times S^{1}$-summand of $\mathcal{H}_{0}$; compare Convention 6.2. Second, the proof that $C F^{-}\left(\mathcal{H}_{0}\right) \cong C F^{-}(\mathcal{H}) \oplus C F^{-}(\mathcal{H})$ (point (2) from the proof of Theorem 6.18) is somewhat harder, requiring a degeneration argument [OSz04a, Proposition 6.4], but for a suitably pinched almost complex structure the isomorphism is still the obvious one and, in particular, is still $\mathbb{Z} / 2$-equivariant (cf. proof of Theorem 1.14).

Corollary 6.19. The numbers $d_{\tau}(K, m)$ are knot invariants.

Proof. This is immediate from Theorem 6.18.

Next we show that the invariants $d_{\tau}(K, i)$ are concordance invariants.

Lemma 6.20. Given an oriented cobordism $T$ from $L_{0}$ to $L_{1}$ there is a map of equivariant Floer complexes $C F_{\mathbb{Z} / 2}^{-}\left(\Sigma\left(L_{1}\right)\right) \rightarrow C F_{\mathbb{Z} / 2}^{-}\left(\Sigma\left(L_{0}\right)\right)$ so that:

- The induced map on the $E^{1}$-page of the spectral sequence induced by the $\theta$-power filtration is

$$
\left(F_{\Sigma(T)}^{*} \otimes \mathbb{I}\right): H F^{-}\left(\Sigma\left(L_{1}\right)\right) \otimes \mathbb{F}_{2}[\theta] \rightarrow H F^{-}\left(\Sigma\left(L_{0}\right)\right) \otimes \mathbb{F}_{2}[\theta]
$$

where $F_{\Sigma(T)}$ is the Ozsváth-Szabó cobordism map associated to $\Sigma(T)$, and

- The map $C F_{\mathbb{Z} / 2}^{-}\left(\Sigma\left(L_{1}\right)\right) \rightarrow C F_{\mathbb{Z} / 2}^{-}\left(\Sigma\left(L_{0}\right)\right)$ has the same effect on gradings as the dual of the Ozsváth-Szabó cobordism map $F_{\Sigma(T)}$.

Proof. Replace all hats by minuses in the proof of Lemma 6.10, and when dealing with births and deaths, work with a suitably pinched almost complex structure (as in the proof of Theorem 6.18).

Theorem 6.21. The numbers $d_{\tau}(K, m)$ are concordance invariants.

Proof. Fix a concordance $T$ from $K_{0}$ to $K_{1}$. Because $\Sigma(T)$ is a rational homology cobordism, by the grading shift formula [OSz06, Theorem 7.1] the map $f_{\Sigma(T)}: C F^{-}\left(\Sigma\left(K_{0}\right), \mathfrak{s}_{0}\right) \rightarrow$ $C F^{-}\left(\Sigma\left(K_{1}\right), \mathfrak{s}_{0}\right)$ induced by the unique spin structure on $\Sigma(T)$ preserves the absolute Maslov grading. Further, by Lemma 6.20, the map $f_{\Sigma(T)}$ induces a map $f_{\Sigma(T)}^{*}: C F_{\mathbb{Z} / 2}^{-}\left(\Sigma\left(K_{1}\right), \mathfrak{s}_{0}\right) \rightarrow$ $C F_{\mathbb{Z} / 2}^{-}\left(\Sigma\left(K_{0}\right), \mathfrak{s}_{0}\right)$ on equivariant Floer cochain complexes. If $E_{s}\left(\Sigma\left(K_{i}\right)\right)$ denotes the $s^{\text {th }}$ page in the spectral sequence associated to the filtration on $C F_{\mathbb{Z} / 2}^{-}\left(\Sigma\left(K_{i}\right), \mathfrak{s}_{0}\right)$ by $\theta$-powers, then the fact that $f_{\Sigma(T)}$ induces an isomorphism on $H F^{\infty}$ implies that

$$
f_{\Sigma(T)}^{*}: E_{1}\left(\Sigma\left(K_{1}\right)\right) / U \text {-torsion } \cong \mathbb{F}_{2}[U, \theta] \rightarrow \mathbb{F}_{2}[U, \theta] \cong E_{1}\left(\Sigma\left(K_{0}\right)\right) / U \text {-torsion }
$$


is injective (or, equivalently, non-zero). (Here, $U$-torsion means the submodule spanned by elements annihilated by $U^{n}$ for some $n$.) From the $\mathbb{F}_{2}[U, \theta]$-module structure and $d_{1}^{2}=0$, it follows that the image of the differential $d_{1}$ is contained in the $U$-torsion submodule of $E_{1}$, so in particular

$$
E_{2}\left(\Sigma\left(K_{i}\right)\right) / U \text {-torsion }=U^{n} \mathbb{F}_{2}[U, \theta] \subseteq \mathbb{F}_{2}[U, \theta] \cong E_{1}\left(\Sigma\left(K_{i}\right)\right)
$$

and so the induced map $f_{\Sigma(T)}^{*}$ on $E_{2} / U$-torsion is injective. Repeating this argument, the induced map on $E_{s} / U$-torsion is injective for all $s$.

Note that

$$
d_{\tau}(K, s)=\min \left\{\operatorname{gr}(x) \mid x \in E_{s}(\Sigma(K)) / U \text {-torsion }\right\}+2 .
$$

Since $f_{\Sigma(T)}^{*}$ is injective on $E_{s} / U$-torsion, we have

$$
\min \left\{\operatorname{gr}(x) \mid x \in E_{s}\left(\Sigma\left(K_{1}\right)\right) / U \text {-torsion }\right\} \geq \min \left\{\operatorname{gr}(x) \mid x \in E_{s}\left(\Sigma\left(K_{0}\right)\right) / U \text {-torsion }\right\},
$$

so $d_{\tau}\left(K_{1}, s\right) \geq d_{\tau}\left(K_{0}, s\right)$. But since the property of being concordant is symmetric, we also have $d_{\tau}\left(K_{0}, s\right) \geq d_{\tau}\left(K_{1}, s\right)$, implying the result.

Remark 6.22. Recall that the spectral sequence (1.7) mentioned in the introduction has the form

$$
\widehat{H F}^{*}(\Sigma(K)) \otimes \mathbb{F}_{2}\left[\theta, \theta^{-1}\right] \otimes V^{\otimes n} \Rightarrow V^{\otimes n} \otimes \mathbb{F}_{2}\left[\theta, \theta^{-1}\right]
$$

for knots (the version for links is slightly more complicated). We do not know if the spectral sequences (6.4) and (1.7) are identified up to tensoring with $V^{\otimes n}$.

Remark 6.23. One can also define a family of integer invariants $q_{\tau}(K, m)$ analogous to $d_{\tau}(K, m)$ but using the spectral sequence associated to the $U$-power filtration instead of the $\theta$-power filtration on $C F_{\mathbb{Z} / 2}^{-}\left(\Sigma(K), \mathfrak{s}_{0}\right)$. These invariants satisfy $q_{\tau}(K)=q_{\tau}(K, 1) \leq$ $q_{\tau}(K, 2) \leq \cdots$.

6.3. Computations. We will concentrate on the homology sphere $\Sigma(2,3,7)$ which is:

(1) The double branched cover of the torus knot $T(3,7)$ (a positive knot).

(2) The double branched cover of the Montesinos knot $M(-1 ;(-2,1),(-3,1),(-7,1))$, which is also the pretzel knot $P(2,-3,-7)$ (a negative knot).

(3) The $(-1)$-surgery of the positive trefoil $T(2,3)$.

$\widehat{H F}(\Sigma(2,3,7))$ is three-dimensional: two-dimensional in grading 0 and one-dimensional in grading -1 ; and $H F^{-}(\Sigma(2,3,7))$ is of the form $\mathbb{F}_{2}[U]\langle\alpha\rangle \oplus \mathbb{F}_{2}\langle\beta\rangle$ with $\operatorname{gr}(\alpha)=\operatorname{gr}(\beta)=$ $-2[\mathrm{OSz} 03 \mathrm{a}$, Equation (25)].

Lemma 6.24. Assume $K \subset S^{3}$ is a knot so that $\widehat{H F}(\Sigma(K))$ is three-dimensional and is supported in two adjacent gradings: two-dimensional in grading $Q$ and one-dimensional in grading $Q-1$. If the involution on the double branched cover $\Sigma(K)$ induces the identity map on $\widehat{H F}(\Sigma(K))$ then $q_{\tau}(K)=2 Q, d_{\tau}(K, 2)=Q$, and $d_{\tau}(m(K), 2)=-Q$. Otherwise, $q_{\tau}(K)=2(Q-1), d_{\tau}(K, 2)=Q$, and $d_{\tau}(m(K), 2)=-Q+2$.

(As before, $m(K)$ denotes the mirror of $K$.)

Proof. We begin with the computation of $q_{\tau}$. Let $\widehat{H F}(\Sigma(K))$ be generated over $\mathbb{F}_{2}$ by two elements $\eta$ and $\nu$ in degree $Q$ and an element $\zeta$ in degree $Q-1$. Even though the isomorphism $\widehat{H F}(K) \simeq \widehat{H F}^{*}(K)$ is not canonical, by looking at a particular Heegaard diagram and 
dualizing the chain complex $\widehat{C F}(\Sigma(K))$ obtained from it, we see that the involution $\tau^{*}$ on $\widehat{H F}^{*}(\Sigma(K))$ is the identity if and only if the involution $\tau_{*}$ on $\widehat{H F}(K)$ is the identity.

First we suppose that $\tau_{*}$ is not the identity on $\widehat{H F}(\Sigma(K))$, so that $\tau^{*}$ is not the identity on $\widehat{H F}^{*}(K)$. Up to change of basis, we may assume that $\tau^{*}(\nu)=\nu+\eta$ and $\tau^{*}(\zeta)=\zeta$. Then in the spectral sequence $\widehat{H F}^{*}(\Sigma(K)) \otimes \mathbb{F}_{2}[\theta] \Rightarrow \widehat{H F}_{\mathbb{Z} / 2}(\Sigma(K))$, the differential $d_{1}=\theta\left(1+\tau^{*}\right)$ on the first page maps $\nu \otimes \theta^{k}$ to $\eta \otimes \theta^{k+1}$ for all $k$. This implies that the $E_{2}$ page of the spectral sequence decomposes as $\mathbb{F}_{2}[\theta]\langle[\zeta]\rangle \oplus\langle[\nu]\rangle$. It follows from grading considerations that the spectral sequence collapses at the $E_{2}$ page, implying that $q_{\tau}(K)=2 \operatorname{deg}\left([\zeta] \otimes \theta^{0}\right)=2(Q-1)$.

Now suppose that $\tau_{*}$ is the identity on $\widehat{H F}(K)$. Then $\tau^{*}$ is also the identity on $\widehat{H F}^{*}(K)$, implying that in the spectral sequence $\widehat{H F}^{*}(\Sigma(K)) \otimes \mathbb{F}_{2}[\theta] \Rightarrow \widehat{H F}_{\mathbb{Z} / 2}(\Sigma(K))$, the differential $d_{1}=\theta\left(1+\tau^{*}\right)$ on the first page is identically zero. Therefore the $E_{2}$ page of this spectral sequence is isomorphic to $\widehat{H F}^{*}(\Sigma(K)) \otimes \mathbb{F}_{2}[\theta]$. Furthermore, we see that for grading reasons, the induced differentials $d_{r}$ on the $E_{r}$ page of the spectral sequence for $r \geq 3$ must be zero. Since we know that $\theta^{-1} \widehat{H F}_{\mathbb{Z} / 2}(\Sigma(K)) \simeq \mathbb{F}_{2}\left[\theta, \theta^{-1}\right]$, this implies that there must be a nonzero differential on the $E_{2}$ page of the spectral sequence. Possibly after a change of basis, we may assume that $d_{2}\left([\eta] \otimes \theta^{k}\right)=[\zeta] \otimes \theta^{k+2}$. This implies that the $E_{3}$ page of the spectral sequence decomposes as the direct sum $\mathbb{F}_{2}[\theta]\langle[\nu]\rangle \oplus\left(\mathbb{F}_{2}[\theta] /\left(\theta^{2}\right)\right)\langle[\zeta]\rangle$. The spectral sequence must collapse at this point, so $q_{\tau}(K)=2 \operatorname{deg}\left([\nu] \otimes \theta^{0}\right)=2 Q$.

Now we turn our attention to $d_{\tau}(K, 2)$ and $d_{\tau}(m(K), 2)$. First, recall that there is a long exact sequence

$$
\cdots \rightarrow H F^{-}(\Sigma(K)) \stackrel{\cdot U}{\rightarrow} H F^{-}(\Sigma(K)) \rightarrow \widehat{H F}(\Sigma(K)) \rightarrow H F^{-}(\Sigma(K)) \rightarrow \cdots
$$

such that the map $H F^{-}(\Sigma(K)) \rightarrow \widehat{H F}(\Sigma(K))$ increases the grading by 2 and the map $\widehat{H F}(\Sigma(K)) \rightarrow H F^{-}(\Sigma(K))$ decreases the grading by 1 [OSz04a, Proposition 2.1]. It follows from this long exact sequence and the gradings for the isomorphism $\widehat{H F}(-Y) \cong \widehat{H F}^{*}(Y)$ that there are non-canonical isomorphisms:

- $H F^{-}(\Sigma(K)) \cong \mathbb{F}_{2}[U]\langle\alpha\rangle \oplus \mathbb{F}_{2}\langle\beta\rangle$, where both $\alpha$ and $\beta$ lie in grading $Q-2$.

- $\widehat{H F}(\Sigma(m(K)))$ is two-dimensional in grading $-Q$ and one-dimensional in grading $-Q+1$.

- $H F^{-}(\Sigma(m(K))) \cong \mathbb{F}_{2}[U]\langle\gamma\rangle \oplus \mathbb{F}_{2}\langle\epsilon\rangle$, where $\gamma$ lies in grading $-Q-2$ and $\epsilon$ lies in grading $-Q-1$.

In particular, $\mathrm{HF}^{-}(\Sigma(m(K)))$ is one-dimensional in each homological grading, implying that $\tau_{*}$ is necessarily the identity on $H^{-}(\Sigma(m(K)))$. From this we conclude that $\tau^{*}$ is the identity map on $H F^{-, *}(\Sigma(K))$, and therefore that $d_{\tau}(K, 2)=d\left(\Sigma(K), \mathfrak{s}_{0}\right)=Q$.

We now turn to $d_{\tau}(m(K), 2)$, which requires a closer look at the long exact sequence (6.25). From the grading shifts, we see that summand of $\widehat{H F}(\Sigma(K))$ in grading $Q$ is exactly the image of the summand of $H F^{-}(\Sigma(K))$ in grading $Q-2$, which is spanned as a vector space by $\alpha$ and $\beta$. Since the long exact sequence (6.25) respects the action $\tau_{*}$, the involution on $\widehat{H F}(\Sigma(K))$ is determined by the involution on $H F^{-}(\Sigma(K))$. There are exactly two $U$ equivariant involutions on $H F^{-}(\Sigma(K))$ : the identity and the involution $\tau_{*}(\alpha)=\alpha+\beta$ and $\tau_{*}(\beta)=\beta$. The first of these induces the identity involution on $\widehat{H F}(\Sigma(K))$, and the second induces the unique nontrivial involution on $\widehat{H F}(\Sigma(K)$. 


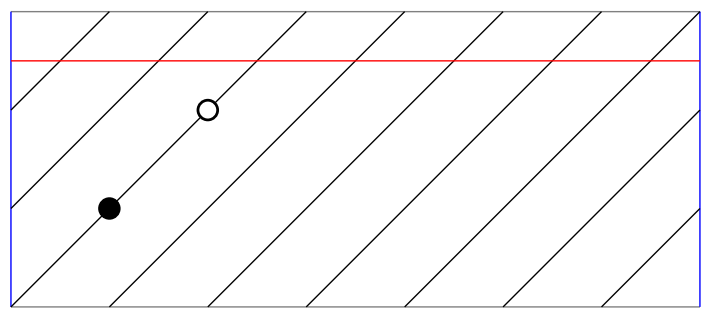

a: The knot $T(3,7)$, drawn with two basepoints on the standard genus one Heegaard diagram of $S^{3}$.

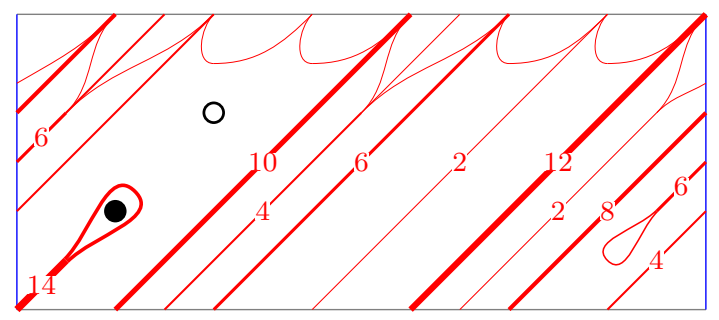

c: Making the previous diagram nice by doing one more finger move.

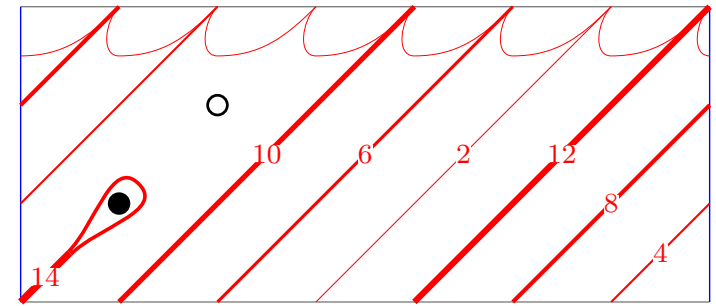

b: A doubly pointed Heegaard diagram for $T(3,7)$ obtained from the previous diagram by doing a finger move along the knot (drawn with train-tracks).

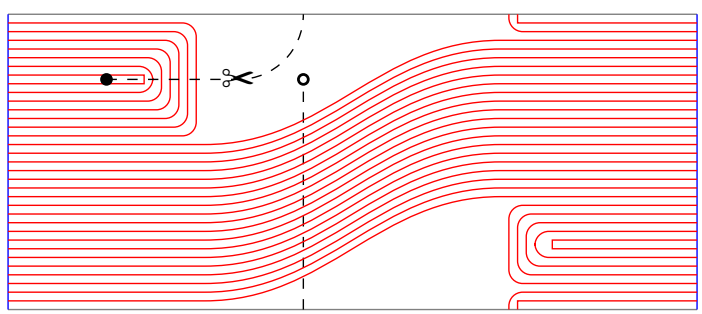

d: The same Heegaard diagram, straightened out, and drawn without train-tracks.

FiguRE 10. A nice Heegaard diagrams for the $(3,7)$ torus knot. In each case, the left and right edges of the rectangle are the $\beta$-circle(s). To construct a nice Heegaard diagram for the double branched cover $\Sigma(2,3,7)$, cut along the dashed line, take two copies, and then join the two copies along the preimages of the dashed line.

This leaves us with two cases. First, suppose that $\tau_{*}$ is the identity map on $\widehat{H F}(\Sigma(K))$. Then $\tau_{*}$ is also the identity map on $H F^{-}(\Sigma(K))$, implying that $\tau^{*}$ is the identity on $H F^{-, *}\left(\Sigma(m(K))\right.$. We conclude that $d_{\tau}(m(K), 2)=d\left(\Sigma(m(K)), \mathfrak{s}_{0}\right)=-Q$.

Finally, suppose that $\tau_{*}$ is nontrivial on $\widehat{H F}(\Sigma(K))$. Then on $H F^{-}(\Sigma(K)), \tau_{*}(\alpha)=\alpha+\beta$ and $\tau_{*}(\beta)=\beta$. This implies that $H F^{-, *}(\Sigma(m(K)))$ has the form $\mathbb{F}_{2}[U]\left\langle\alpha^{\prime}\right\rangle \oplus\left\langle\beta^{\prime}\right\rangle$ such that $\operatorname{gr}\left(\alpha^{\prime}\right)=\operatorname{gr}\left(\beta^{\prime}\right)=-(Q-2)-4=-Q-2$, and the involution is $\tau^{*}\left(\alpha^{\prime}\right)=\alpha^{\prime}+\beta^{\prime}$ and $\tau^{*}\left(\beta^{\prime}\right)=\beta^{\prime}$. In particular, $\left(1+\tau_{*}\right)\left(\alpha^{\prime}\right)=\beta^{\prime}$, so on the first page of the spectral sequence of Figure $9, \theta^{k} \alpha^{\prime}$ cancels with $\theta^{k+1} \beta^{\prime}$ for all $k \geq 0$. This implies that the second page of the spectral sequence of Figure 9 is the direct sum of a free $\mathbb{F}_{2}[U, \theta]$-summand generated by $\left[U \alpha^{\prime}\right]$ and a single $\mathbb{F}_{2}$-summand generated by $\left[\beta^{\prime}\right]$, which lies in grading $(-Q-2)+2=-Q$. Thus $d_{\tau}(m(K), 2)=-Q+2$.

In view of Lemma 6.24 , in order to compute these invariants for $T(3,7)$ or $P(2,-3,-7)$, it is enough to compute the corresponding $\mathbb{Z} / 2$-actions on $\widehat{H F}(\Sigma(2,3,7))$. Thanks to [JT12], we are free to choose any Heegaard diagram, which is invariant under this $\mathbb{Z} / 2$-action in order to compute the induced map on $\widehat{H F}$. We carry out the computations for the two $\mathbb{Z} / 2$-actions with two $\mathbb{Z} / 2$-equivariant Heegaard diagrams in following two propositions. 
Proposition 6.26. The involution of $\widehat{H F}(\Sigma(2,3,7))$ that comes from viewing $\Sigma(2,3,7)$ as the double branched cover of $T(3,7)$ is the identity map. Hence, $q_{\tau}(T(3,7))=d_{\tau}(T(3,7), 2)=$ $d_{\tau}(-T(3,7), 2)=0$.

Proof. For this computation, we resort to the technique of nice diagrams. We first choose a doubly pointed Heegaard diagram for the knot $T(3,7) \subset S^{3}$, satisfying the following two conditions:

- The diagram is nice in the sense of [SW10]; i.e., every region that does not contain a $z$ or a $w$ basepoint is either a bigon or a rectangle.

- The region containing the basepoint $z$ is a bigon.

Given such a doubly nice Heegaard diagram, the induced $\mathbb{Z} / 2$-equivariant Heegaard diagram for $\Sigma(T(3,7))=\Sigma(2,3,7)$ (see Section 5.1), with basepoint the preimage of $w$, is nice. Thus, it is a combinatorial exercise to compute the chain complex $\widehat{C F}(\Sigma(2,3,7))$, and the $\mathbb{Z} / 2$-action on it from such a diagram (compare Section 5.2).

One can construct a doubly nice Heegaard diagram for any knot $K$ as follows. Start with any doubly pointed Heegaard diagram for $K$. Ensure that $z$ and $w$ are separated by a single $\alpha$-circle by adding a handle between the regions containing $z$ and $w$ if necessary. Then run the Sarkar-Wang algorithm to make this Heegaard diagram nice with respect to the basepoint $w$. Recall that one may run the algorithm without needing to stabilize or destabilize the Heegaard diagram, and by only isotoping and handlesliding the $\beta$-circles (and keeping the $\alpha$-circles fixed). Therefore, for the resulting nice diagram, we may still assume that the $z$ and the $w$ basepoint are separated by a single $\alpha$-circle. Let $R$ be the region in this new Heegaard diagram that contains $z$. If $R$ is a bigon, we are already done. Otherwise, if $R$ is a rectangle, draw an arc from $z$ to $w$ in the complement of the $\alpha$-circles intersecting the $\beta$-multicurve minimally. Then perform a finger move with the $\alpha$-arc that separates $z$ and $w$ along this arc; this cuts $R$ into a rectangle and a bigon, and we may, trivially, ensure that $z$ ends up in the bigon.

In our case, we do not have to resort to the above general algorithm, which tends to produce unwieldy diagrams. Instead, we use the strategy outlined in Figure 10. We first draw $T(3,7)$ on the standard genus-one Heegaard diagram for $S^{3}$ as a union of two embedded arcs joining two basepoints, so that the first arc lies in the complement of the $\beta$-circle; see Figure 10a (as usual, red is $\alpha$ and blue is $\beta$ ). Then we perform a finger move on the $\alpha$ circle along the other arc, producing a doubly pointed Heegaard diagram for $T(3,7)$; see Figure $10 \mathrm{~b}$. This is not a nice Heegaard diagram: it has a single bad region which is a hexagon. Performing one more finger move makes this diagram doubly nice; see Figures 10c-10d.

As per the above discussion, if $\mathcal{H}$ denotes the double branched cover of this doubly nice diagram, we can compute $\widehat{C F}(\mathcal{H})$ and the $\mathbb{Z} / 2$-action combinatorially. We use the computer program at https://github.com/sucharit/hf-hat to study $\mathcal{H}$. The complex $\widehat{C F}(\mathcal{H})$ has 545 generators, and its homology $H_{*}(\widehat{C F}(\mathcal{H}))=\widehat{H F}(\Sigma(2,3,7))$ is three-dimensional. To compute the $\mathbb{Z} / 2$-action, say $\tau$, on $\widehat{H F}(\Sigma(2,3,7))$, we compute the homology of the mapping cone of $\left(\mathbb{I}+\tau_{\#}\right)$, where $\tau_{\#}: \widehat{C F}(\mathcal{H}) \rightarrow \widehat{C F}(\mathcal{H})$ is the chain-level $\mathbb{Z} / 2$-action. The homology of Cone $\left(\mathbb{I}+\tau_{\#}\right)$ is six-dimensional, which implies that $\tau: \widehat{H F}(\Sigma(2,3,7)) \rightarrow \widehat{H F}(\Sigma(2,3,7))$ is the identity map. 


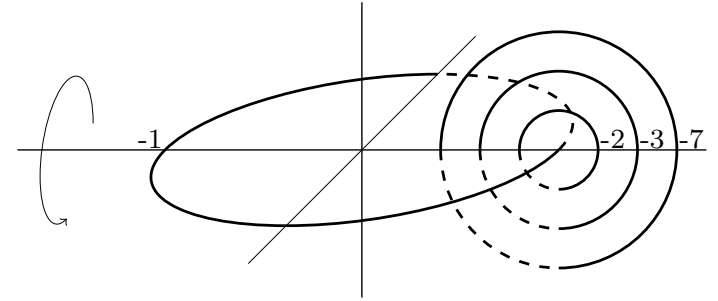

a: A surgery presentation of $\Sigma(2,3,7)$ that exhibits a $\mathbb{Z} / 2$ symmetry.

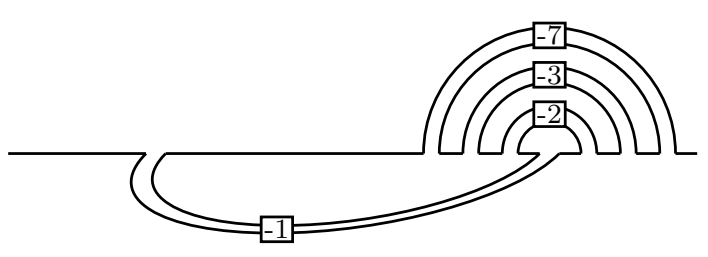

c: The fixed set in the quotient $S^{3}$ is the Montesinos knot $M(-1 ;(-2,1),(-3,1),(-7,1))$.

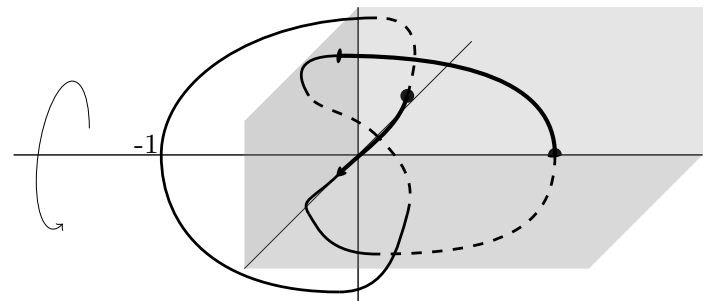

b: Using $\mathbb{Z} / 2$-equivariant Kirby moves, it is also the (-1)-surgery on the positive trefoil (the portion of the trefoil in the positive octant is emphasized.)

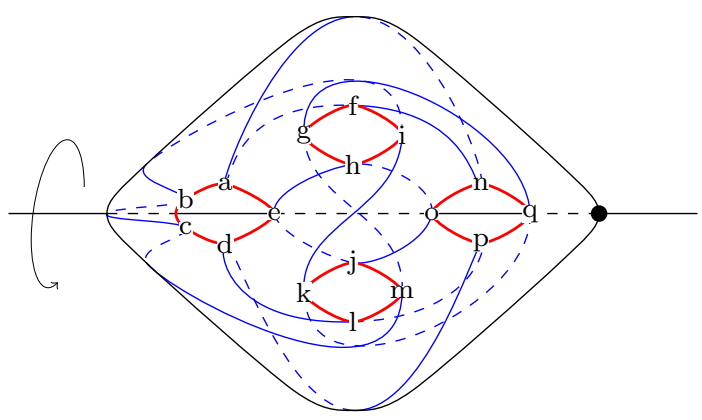

d: A Heegaard diagram for the $(-1)$ surgery of the trefoil, along with the $\mathbb{Z} / 2$ symmetry. The small planar circles are $\alpha$, and the large non-planar ones are $\beta$.

Figure 11. A Heegaard diagram for $\Sigma(2,3,7)$, viewed as a (-1)-surgery of the positive trefoil. The diagram reflects the $\mathbb{Z} / 2$ symmetry that comes from viewing $\Sigma(2,3,7)$ as the double branched cover of the Montesinos knot $M(-1 ;(-2,1),(-3,1),(-7,1))$.

Proposition 6.27. The involution of $\widehat{H F}(\Sigma(2,3,7))$ that comes from viewing $\Sigma(2,3,7)$ as the double branched cover of $P(2,-3,-7)$ is not the identity map. Hence, $q_{\tau}(P(2,-3,-7))=$ $-2, d_{\tau}(P(2,-3,-7), 2)=0$, and $d_{\tau}(P(-2,3,7), 2)=2$.

Proof. We carry out this computation directly by hand. It is well-known that the following $\mathbb{Z} / 2$-actions on $\Sigma(2,3,7)$ are the same:

- The $\mathbb{Z} / 2$-action coming from viewing $\Sigma(2,3,7)$ as $\Sigma(P(2,-3,-7))$.

- The $\mathbb{Z} / 2$-action coming from the description of $\Sigma(2,3,7)$ as $(-1)$-surgery on the trefoil $T(2,3)$ and applying the unique strong inversion (orientation-reversing involution) of $T(2,3)$.

See, for instance, Watson [Wat10]; see also Figures 11a-11c.

Therefore, we start with a $\mathbb{Z} / 2$-equivariant Heegaard diagram $\mathcal{H}$ for the $(-1)$-surgery on $T(2,3)$ in Figure 11d, where as usual, red denotes $\alpha$ and blue denotes $\beta$. (Note that the Heegaard surface is oriented as the boundary of the $\alpha$-handlebody, so it is oriented as the boundary of the 'outside'.) We have labeled the seventeen intersection points between the 
$\alpha$-circles and the $\beta$-circles a, b, .., p, q. As in Point (ET-2) of Section 4, the Lagrangians $T_{\alpha}, T_{\beta} \subset \operatorname{Sym}^{4}(\Sigma)$ satisfy Hypothesis 4.1 , so we may compute the equivariant Floer complex as $\operatorname{RHom}_{\mathbb{F}_{2}[\mathbb{Z} / 2]}\left(\widehat{C F}(\mathcal{H}), \mathbb{F}_{2}\right)$, where $\widehat{C F}(\mathcal{H})$ is computed with respect to a generic $\mathbb{Z} / 2$ equivariant path of almost complex structures on $\operatorname{Sym}^{g}(\Sigma)$. With respect to such a path, the chain complex $\widehat{C F}(\mathcal{H})$ has the following form:

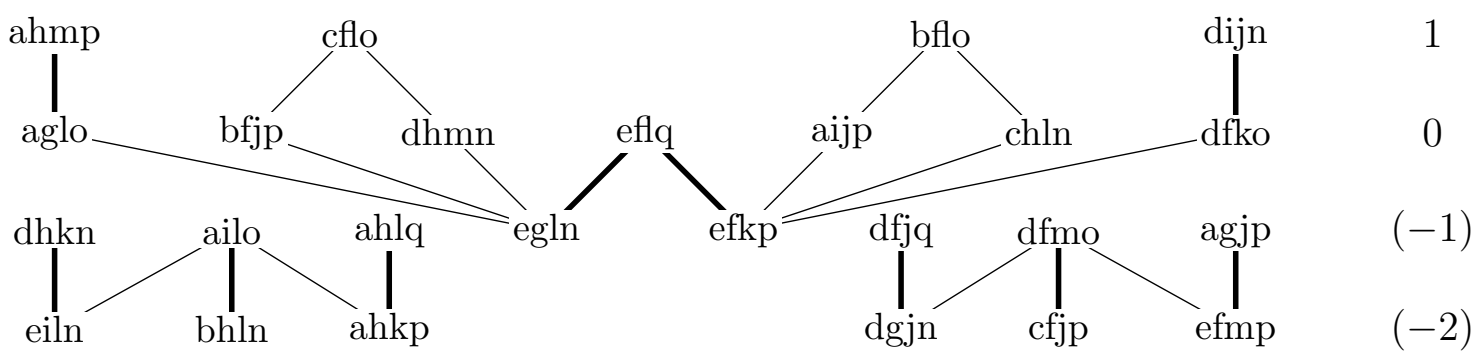

Here, a line (thick or thin) indicates the presence of a positive Maslov index-one domain, the only domains which may contribute to the differential. A thick line indicates that the domain is a $2 n$-gon, and therefore, necessarily contributes 1 to the $\widehat{C F}$ differential. The $\mathbb{Z} / 2$-action is reflection along the central vertical line.

The fact that $\partial^{2}=0$ implies that egln does not occur in $\partial$ (aglo) and efkp does not appear in $\partial$ (dfko). So, this complex decomposes into two $\mathbb{Z} / 2$-equivariant summands, so that one of them is the following, and other one is acyclic.

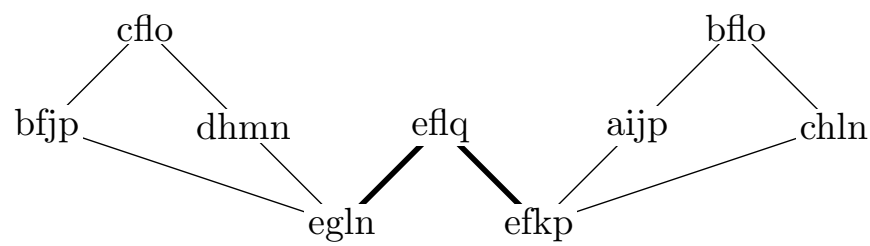

The gradings on the right are a priori just relative gradings, well-defined up to some additive constant. However, from the form of the homology $\widehat{H F}(\Sigma(2,3,7))$, we see that these gradings are actually the correct gradings.

Since $\widehat{H F}(\Sigma(2,3,7))$ is zero-dimensional in grading 1 , we must have both $\partial(\mathrm{cflo})$ and $\partial$ (bflo) non-zero. And since $\widehat{H F}(\Sigma(2,3,7))$ is one-dimensional in grading $(-1)$, we must have $\partial(\mathrm{bfjp})=\partial(\mathrm{dhmn})=\partial($ aijp $)=\partial(\operatorname{chln})=0$. Therefore, the two-dimensional homology in grading 0 is generated by $\langle$ bfjp, dhmn $\rangle / \partial$ (cflo) and $\langle$ aijp, chln $\rangle / \partial$ (bflo). The $\mathbb{Z} / 2$-action interchanges these two generators, and is therefore, non-trivial.

Our results summarize as follows:

$$
\begin{array}{ll}
q_{\tau}(T(3,7))=0 & d_{\tau}(T(3,7), 2)=0 \\
q_{\tau}(T(-3,7))=0 & d_{\tau}(T(-3,7), 2)=0 \\
q_{\tau}(P(2,-3,-7))=-2 & d_{\tau}(P(2,-3,-7), 2)=0 \\
q_{\tau}(P(-2,3,7))=2 & d_{\tau}(P(-2,3,7), 2)=2
\end{array}
$$

We deduce a number of corollaries.

Corollary 6.28. The actions $\tau_{*}$ on $\widehat{H F}(\Sigma(K)), H^{-}(\Sigma(K))$, and $H^{+}(\Sigma(K))$ coming from the double-branched cover involution on $\Sigma(K)$ are not determined by the 3-manifold $\Sigma(K)$. 
Corollary 6.29. The knot invariant $q_{\tau}(K)$ is not determined by 3-manifold $\Sigma(K)$.

Furthermore, our examples show that $q_{\tau}(K)$ is not the same as three other major concordance invariants arising from knot homology theories.

Corollary 6.30. The knot invariant $q_{\tau}(K)$ is not identified, even after scaling, with any of the Ozsváth-Szabó concordance invariant $\tau(K)$ [OSz03b, Ras03], the Rasmussen invariant $s(K)$ [Ras10], or the Manolescu-Owens invariant $\delta(K)$ [MO07].

Proof. We have $s(T(3,7))=2 \tau(T(3,7))=12$ and $s(P(2,-3,-7))=2 \tau(P(2,-3,-7)=$ -10 [Ras10, Theorem 4]. Furthermore, $\delta(T(3,7))=\delta(P(2,-3,-7))=2 d(\Sigma(2,3,7))=0$ [OSz03a, Subsection 8.1]. On the other hand, for the right-handed trefoil, $q_{\tau}(T(2,3))=$ $\tau(T(2,3))=s(T(2,3)) / 2=\delta(T(2,3))=1$.

The Heegaard Floer homology of a three-manifold also carries a conjugation symmetry

$$
\iota_{*}: \widehat{H F}(Y, \mathfrak{s}) \rightarrow \widehat{H F}(Y, \overline{\mathfrak{s}})
$$

induced by a sequence of Heegaard moves to a Heegaard diagram $\mathcal{H}=(\Sigma, \boldsymbol{\alpha}, \boldsymbol{\beta}, z)$ for $Y$ from its conjugate $\overline{\mathcal{H}}=(-\Sigma, \boldsymbol{\alpha}, \boldsymbol{\beta}, z)$ [OSz04a, Theorem 2.4]. A similar action exists for the other variants of Heegaard Floer homology.

Corollary 6.31. There are knots $K$ for which the actions $\tau_{*}$ and $\iota_{*}$ on $\widehat{H F}(\Sigma(K))$ are different. The same holds with $\widehat{H F}(\Sigma(K))$ replaced by $\mathrm{HF}^{+}(\Sigma(K))$ or $\mathrm{HF}^{-}(\Sigma(K))$.

In [HM15], Hendricks and Manolescu use the conjugation symmetry to associate to a three manifold $Y$ with spin structure $\mathfrak{s}$ two homology cobordism invariants $\underline{d}(Y, s)$ and $\bar{d}(Y, s)$. The second of these is defined extremely formally similarly to $d_{\tau}(K, 2)$.

Corollary 6.32. The invariants $\bar{d}\left(\Sigma(K), \mathfrak{s}_{0}\right)$ and $d_{\tau}(K, 2)$ are not identified.

Proof. We have $\bar{d}(-\Sigma(2,3,7))=2[$ HM15, Section 6.8].

\section{Spectral Sequences from symplectic Khovanov homology}

Khovanov homology admits a number of deformations [Lee05, BN05, OSz05, Sza15, SSS14]; some of these are believed to correspond, at least philosophically, to actions of $U(1)$ or $\mathbb{Z} / 2$. On the Floer-theoretic side, it is easy to see (and well known by experts) that the manifolds used to construct symplectic Khovanov homology admits an $O(2)$-symmetry; see Section 7.1. The action of a reflection $\mathbb{Z} / 2 \subset O(2)$ on symplectic Khovanov homology was studied by Seidel-Smith [SS10]; see also Section 7.6. The main goal of this section is to develop some properties of the action of more general finite subgroups of $O(2)$ in the symplectic setting. We start by reviewing the definition of symplectic Khovanov homology and the group actions on it in Section 7.1 and some notations for the cohomology of cyclic and dihedral groups in Section 7.2. We then prove a localization result for some of these equivariant cohomologies in Section 7.3, and prove that all of these equivariant cohomologies are link invariants in Section 7.4. Section 7.5 is a brief digression, to prove that Manolescu's reduced symplectic Khovanov homology is also an invariant of based links. We conclude with some speculations about the relationship between these constructions and various combinatorial deformations in Section 7.6.

As in the rest of this paper, all cohomology groups have coefficients in $\mathbb{F}_{2}$, unless otherwise noted. 
7.1. A brief review of symplectic Khovanov homology. We start by reviewing the construction of Seidel-Smith's symplectic Khovanov homology [SS06], largely from the perspective of Manolescu's reformulation [Man06].

As in Section 6, fix a bridge diagram for a link $L$, consisting of $\operatorname{arcs} A_{i}$ and $B_{i}$ with endpoints $\left\{p_{1}, \ldots, p_{2 n}\right\}$. Let $p(z)=\left(z-p_{1}\right) \cdots\left(z-p_{2 n}\right)$ and consider the affine algebraic surface

$$
S=\left\{(z, u, v) \in \mathbb{C}^{3} \mid u^{2}+v^{2}+p(z)=0\right\} .
$$

The ring of regular functions on $S$ is, of course,

$$
R=\mathbb{C}[z, u, v] /\left(u^{2}+v^{2}+p(z)\right) .
$$

Let $\operatorname{Hilb}^{n}(S)$ denote the Hilbert scheme of $n$-tuples of points in $S$ or, more precisely, length $n$ closed subschemes of $S$. In particular, a point in $\operatorname{Hilb}^{n}(S)$ is an ideal $I \subset R$ so that $\operatorname{dim}_{\mathbb{C}}(R / I)=n$. For example, given $n$ distinct points $q_{1}=\left(z_{1}, u_{1}, v_{1}\right), \ldots, q_{n}=\left(z_{n}, u_{n}, v_{n}\right)$ of $S$, let $I_{j}=\left(z-z_{j}, u-u_{j}, v-v_{j}\right) \subset R$ and let $I=I_{1} \cdots I_{n}$ be the product of these ideals. Then $I \in \operatorname{Hilb}^{n}(S)$. Thus, we have an embedding $\operatorname{Sym}^{n}(S) \backslash \Delta \hookrightarrow \operatorname{Hilb}^{n}(S)$; it turns out that the image is open. We call the complement of this embedding the diagonal in $\operatorname{Hilb}^{n}(S)$, and denote it by $\Delta$.

Consider the map $i: S \rightarrow \mathbb{C},(z, u, v) \mapsto z$, which corresponds to the obvious map $\mathbb{C}[z] \hookrightarrow$ $\mathbb{C}[z, u, v] \rightarrow R$. Let $R_{1}$ denote the image of $\mathbb{C}[z]$ in $R$. The intersection of the ideal $\left(u^{2}+\right.$ $\left.v^{2}+p(z)\right)$ with $\mathbb{C}[z] \subset \mathbb{C}[z, u, v]$ is trivial, so $R_{1} \cong \mathbb{C}[z]$. Given an ideal $I \in \operatorname{Hilb}^{n}(\mathbb{C})$, let $i(I)=I \cap R_{1} \subset R_{1}$. Let

$$
\mathcal{Y}_{n}=\left\{I \in \operatorname{Hilb}^{n}(S) \mid i(I) \text { has length } n\right\} .
$$

Manolescu shows that the space $\mathcal{Y}_{n}$ is biholomorphic to the space $\mathcal{Y}_{n, \tau}$ considered by SeidelSmith [Man06, Proposition 2.7].

Each of the $\operatorname{arcs} A_{i}$ and $B_{i}$ gives a totally real $S^{2}$ in $S$, by:

$$
\begin{aligned}
& \Sigma_{A_{i}}=\left\{(z, u, v) \in S \mid z \in A_{i}, u, v \in \sqrt{-p(z)} \mathbb{R}\right\} \\
& \Sigma_{B_{i}}=\left\{(z, u, v) \in S \mid z \in B_{i}, u, v \in \sqrt{-p(z)} \mathbb{R}\right\} .
\end{aligned}
$$

[Man06, p. 2]. These spheres give submanifolds

$$
\begin{aligned}
& \mathcal{K}_{A}=\Sigma_{A_{1}} \times \cdots \times \Sigma_{A_{n}} \subset\left(\operatorname{Sym}^{n}(S) \backslash \Delta\right) \subset \operatorname{Hilb}^{n}(S) \\
& \mathcal{K}_{B}=\Sigma_{B_{1}} \times \cdots \times \Sigma_{B_{n}} \subset\left(\operatorname{Sym}^{n}(S) \backslash \Delta\right) \subset \operatorname{Hilb}^{n}(S) .
\end{aligned}
$$

There is a natural Kähler form on $\mathcal{Y}_{n}$ for which $\mathcal{K}_{A}$ and $\mathcal{K}_{B}$ are Lagrangian [Man06, Theorem $1.2]$.

The Lagrangians $\mathcal{K}_{A}$ and $\mathcal{K}_{B}$ do not intersect transversely. To define their Floer homology one needs to either perturb one of them slightly, or work in the more general setting of Lagrangians with clean intersections [Poź99] (as Manolescu does [Man06, Section 6.1]); the approaches give isomorphic Floer homology groups. Manolescu shows that the Floer cohomology $H F\left(\mathcal{K}_{A}, \mathcal{K}_{B}\right)$ inside $\mathcal{Y}_{n}$ is the symplectic Khovanov homology $K_{\text {symp }}(L)$ [Man06, Theorem 1.2].

There is also a reduced version of the construction, giving a reduced symplectic Khovanov homology $\widetilde{K h}$ symp, where we consider the variety

$$
\widetilde{\mathcal{Y}}_{n}=\left\{I \in \operatorname{Hilb}^{n-1}(S) \mid i(I) \text { has length } n-1\right\} .
$$


and consider the Floer homology of $\widetilde{\mathcal{K}}_{A}=\Sigma_{A_{1}} \times \cdots \times \Sigma_{A_{n-1}}$ and $\widetilde{\mathcal{K}}_{B}=\Sigma_{B_{1}} \times \cdots \times$ $\Sigma_{B_{n-1}}[$ Man06, Section 7.5]. We will return to the reduced theory in Section 7.5.

Convention 7.2. The combinatorial Khovanov complex is of cohomological type, i.e., the differential raises the homological grading by 1. Correspondingly, Seidel-Smith constructed symplectic Khovanov homology as a Floer cohomology group. We will continue to work with the Floer chain (rather than cochain) complex of $\mathcal{K}_{A}$ and $\mathcal{K}_{B}$, so the cohomology of our complexes gives Seidel-Smith's symplectic Khovanov homology.

7.1.1. The group action. There is an action of the orthogonal group $O(2, \mathbb{C}) \subset G L(2, \mathbb{C})$ on $S$ induced from the action on $\mathbb{C}\langle u, v\rangle=\mathbb{C}^{2}$, and a corresponding action of $O(2, \mathbb{C})$ on $\operatorname{Hilb}^{n}(S)$. The subgroup $O(2):=O(2, \mathbb{R}) \subset O(2, \mathbb{C})$ on $S$ preserves the spheres $\Sigma_{A_{i}}$ and $\Sigma_{B_{i}}$, and hence the action of $O(2)$ on $\operatorname{Hilb}^{n}(S)$ preserves the Lagrangians $\mathcal{K}_{A}$ and $\mathcal{K}_{B}$.

Lemma 7.3. The $O(2)$-action on $\operatorname{Hilb}^{n}(S)$ preserves the open subset $\mathcal{Y}_{n}$.

Proof. Elements of $O(2)$ fix $R_{1}$, and hence do not change $i(I)$ or, in particular, the length of $i(I)$.

Instead of focusing on the action of $O(2)$, we will restrict our attention to the dihedral group of order $2^{m+1}, D_{2^{m}}=\left\langle\sigma, \tau \mid \sigma^{2^{m}}, \tau^{2}, \sigma \tau \sigma \tau\right\rangle$, acting on $S$ as follows:

$$
\sigma(z, u, v)=\left(z, u \cos \theta_{m}-v \sin \theta_{m}, u \sin \theta_{m}+v \cos \theta_{m}\right) \quad \tau(z, u, v)=(z, u,-v),
$$

where $\theta_{m}=2 \pi / 2^{m}$.

Lemma 7.5. The action of $D_{2^{m}}$ on $\mathcal{Y}_{n}$ preserves $\mathcal{K}_{A}$ and $\mathcal{K}_{B}$. Moreover, one can choose a small Hamiltonian perturbation $\mathcal{K}_{A}^{\prime}$ of $\mathcal{K}_{A}$ so that $\mathcal{K}_{A}^{\prime}$ is transverse to $\mathcal{K}_{B}$ and is still preserved by the $D_{2^{m}}$-action.

Proof. The first statement is immediate from the definitions of $\mathcal{K}_{A}$ and $\mathcal{K}_{B}$. For the second statement, recall the map $i: S \rightarrow \mathbb{C}$. There are two kinds of intersections between the spheres $\Sigma_{A_{j}}$ and $\Sigma_{B_{k}}$ :

(i-1) The first kind consists of intersections which lie over a point $p_{\ell} \in\left\{1, \ldots, p_{2 n}\right\}$, i.e., intersections corresponding to endpoints of the arcs, i.e., points of $\Sigma_{A_{j}} \cap \Sigma_{B_{k}} \cap i^{-1}\left(p_{\ell}\right)$. These intersections are already transverse.

(i-2) The second kind consists of intersections which lie over a point $q \notin\left\{1, \ldots, p_{2 n}\right\}$, i.e., points of $\left(\Sigma_{A_{j}} \cap \Sigma_{B_{k}} \cap i^{-1}(q)\right) \cong S^{1}$. The action of $D_{2^{m}}$ on each such circle of intersections is the usual action of $D_{2^{m}} \subset O(2)$ on $S^{1} \subset \mathbb{C}$. Choose a symplectic identification of a neighborhood of this $S^{1}$ with $[-1,1] \times[-1,1] \times T^{*} S^{1}=\{(x, y, \theta, t)\}$ (with symplectic form $d x \wedge d y+d \theta \wedge d t)$, so that $i(x, y, \theta, t)=x+i y, q$ is identified with the origin, the $O(2)$-action is on the $\theta$ coordinate, $\Sigma_{A_{j}}$ is $\{(x, 0, \theta, 0)\}, \Sigma_{B_{k}}$ is $\{(0, y, \theta, 0)\}$, and in terms of $(z, u, v)$-coordinates the subspace $\{(x, y, \theta, 0)\}$ is embedded as

$$
z=x+i y \quad u=\gamma \cos \theta \quad v=\gamma \sin \theta
$$

where $\gamma$ is chosen to be one of the two (distinct) square roots of $-p(z)$. Choose a bump function $\phi:[-1,1] \rightarrow \mathbb{R}$ which is 1 on $(-1 / 2,1 / 2)$ and 0 outside $(-3 / 4,3 / 4)$. Consider the Hamiltonian

$$
H(x, y, \theta, t)=\phi(x) \phi(y) \phi(t) \cos \left(2^{m} \theta\right) .
$$


Let $\Sigma_{A_{j}}^{\prime}$ be the image of $\Sigma_{A_{j}}$ under a short-time flow of the Hamiltonian vector field

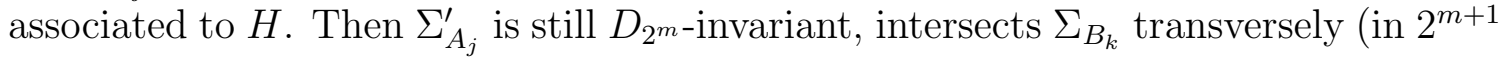
points), and the projection $i\left(\Sigma_{A_{j}}^{\prime}\right)$ is still $A_{j}$.

Choosing a perturbation as in Point (i-2) for each of the second kind of intersections and taking the product of the corresponding Lagrangians $\Sigma_{A_{j}}^{\prime}$ gives the desired $\mathcal{K}_{A}^{\prime}$.

We can define a freed Floer complex over $D_{2^{m}}, \mathcal{C}_{K h}^{\text {symp,free }}(L):=\widetilde{C F}^{D_{2}^{m}}\left(\mathcal{K}_{A}^{\prime}, \mathcal{K}_{B}\right)$, by applying the construction of Section 3.7 to the perturbation from Lemma 7.5. We can also restrict to $\mathbb{Z} / 2^{m} \subset D_{2^{m}}$, giving a freed Floer complex $\widetilde{C F} \mathbb{Z} / 2^{m}\left(\mathcal{K}_{A}^{\prime}, \mathcal{K}_{B}\right)$. In the special case $m=1$, there are two nonequivalent $\mathbb{Z} / 2$-actions: the action by rotation by $\pi$, denoted $\sigma$, and the reflection, $\tau$; when we talk about $\widetilde{C F^{\mathbb{Z} / 2}}\left(\mathcal{K}_{A}^{\prime}, \mathcal{K}_{B}\right)$ we will make it clear in the text which action is under consideration. We also obtain equivariant Floer complexes and homologies

$$
\begin{aligned}
& C F_{G}\left(\mathcal{K}_{A}^{\prime}, \mathcal{K}_{B}\right):=\operatorname{RHom}_{\mathbb{F}_{2}[G]}\left(\widetilde{C F}^{G}\left(\mathcal{K}_{A}^{\prime}, \mathcal{K}_{B}\right), \mathbb{F}_{2}\right) \\
& H F_{G}\left(\mathcal{K}_{A}^{\prime}, \mathcal{K}_{B}\right):=H_{*}\left(C F_{G}\left(\mathcal{K}_{A}^{\prime}, \mathcal{K}_{B}\right)\right),
\end{aligned}
$$

for $G=D_{2^{m}}$ or $\mathbb{Z} / 2^{m}$, which are modules over $H^{*}(G)$. There are spectral sequences from $H^{*}\left(G ; H F\left(\mathcal{K}_{A}^{\prime}, \mathcal{K}_{B}\right)\right) \Rightarrow H F_{G}\left(\mathcal{K}_{A}^{\prime}, \mathcal{K}_{B}\right)$. There is a quasi-isomorphism $\widetilde{C F^{\mathbb{Z} / 2^{m}}}\left(\mathcal{K}_{A}^{\prime}, \mathcal{K}_{B}\right) \cong$ $\widetilde{C F}{ }^{D_{2} m}\left(\mathcal{K}_{A}^{\prime}, \mathcal{K}_{B}\right)$ over $\mathbb{F}_{2}\left[\mathbb{Z} / 2^{m}\right] ;$ furthermore, for any $m^{\prime} \geq m$, there is a quasi-isomorphism $\widetilde{C F} D^{D^{m}}\left(\mathcal{K}_{A}^{\prime}, \mathcal{K}_{B}\right) \cong \widetilde{C F} D_{2^{m^{\prime}}}\left(\mathcal{K}_{A}^{\prime}, \mathcal{K}_{B}\right)$ over $\mathbb{F}_{2}\left[D_{2^{m}}\right]$. It follows from Theorem 1.26, proved in Section 7.4, that all of this data is an invariant of the link $L$.

7.1.2. Homotopy classes and gradings. Next, we see that the action of $D_{2^{m}}$ preserves the grading on the symplectic Khovanov complex. Although there is a more direct argument (see Lemma 7.17), we will use a lemma about the path space which seems of independent interest. Recall that the Heegaard Floer homology groups decompose according to $\operatorname{spin}^{c}$ structures, which correspond to the homotopy classes of paths between the Lagrangians $T_{\alpha}$ and $T_{\beta}$. There is no corresponding decomposition of symplectic Khovanov homology:

Lemma 7.6. The space $\mathcal{Y}_{n}$ is simply connected. In particular, the space of paths from $\mathcal{K}_{A}$ to $\mathcal{K}_{B}$ is path connected.

This lemma and its proof was communicated to us by Mohammed Abouzaid and Ivan Smith.

Proof. The proof of simple-connectivity is essentially the same as Seidel-Smith's proof that $H^{1}\left(\mathcal{Y}_{n}\right)=0$ [SS06, Lemma 44]: as explained there (see also [SS06, Section 2.3]), $\mathcal{Y}_{n}$ is diffeomorphic to the $(n, n)$ Springer variety $\mathfrak{B}_{n, n}$. Russell-Tymoczko [RT11, Theorem 5.5] and Wehrli [Weh09, Theorem 1.2] showed that $\mathfrak{B}_{n, n}$ is homeomorphic to the space $\widetilde{S}:=$ $\cup_{a} S_{a} \subset\left(\mathbb{C} P^{1}\right)^{2 n}$ where the union is over all isotopy classes $a$ of upper half-plane crossingless matchings $a$, viewed as involutions $a:\{1, \ldots, 2 n\} \rightarrow\{1, \ldots, 2 n\}$ and

$$
S_{a}:=\left\{\left(l_{1}, \ldots, l_{2 n}\right) \in\left(\mathbb{C} P^{1}\right)^{2 n} \mid \forall j l_{j}=l_{a(j)}\right\} .
$$

This is a union of simply connected sets, all of the pairwise intersections of which are connected, so by the Seifert-van Kampen theorem, $\widetilde{S} \cong \mathfrak{B}_{n, n} \cong \mathcal{Y}_{n}$ is simply connected. Now, the fact that the path space is connected follows from the path-loop fibration

$$
\Omega \mathcal{Y}_{n} \rightarrow \mathcal{P}\left(\mathcal{K}_{A}, \mathcal{K}_{B}\right) \rightarrow \mathcal{K}_{A} \times \mathcal{K}_{B}
$$


Proposition 7.7. The action of $D_{2^{m}}$ on $\left(\mathcal{Y}_{n}, \mathcal{K}_{A}^{\prime}, \mathcal{K}_{B}\right)$ preserves the absolute (homological) grading.

Proof. By Lemma 7.6, any two generators $\mathbf{x}, \mathbf{y} \in \mathcal{K}_{A}^{\prime} \cap \mathcal{K}_{B}$ can be connected by a homotopy class of Whitney disks $\phi \in \pi_{2}(\mathbf{x}, \mathbf{y})$. Since $D_{2^{m}}$ acts by symplectomorphisms, the Maslov

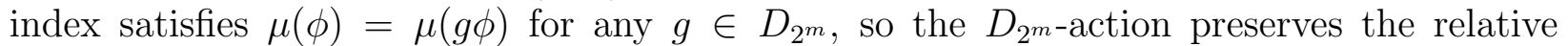
Maslov gradings. Since there is a $D_{2^{m}}$-fixed generator (in fact, at least two fixed generators;

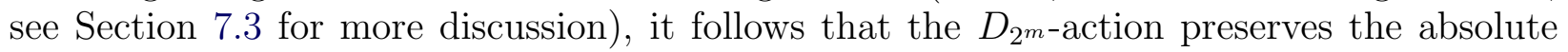
grading, as well.

7.1.3. The cylindrical formulation. To visualize holomorphic disks in $\left(\mathcal{Y}_{n}, \mathcal{K}_{A}^{\prime}, \mathcal{K}_{B}\right)$ it will be convenient to use the following six-dimensional formulation; compare [AS16, Section 5.8]. First, an intersection point between $\mathcal{K}_{A}$ and $\mathcal{K}_{B}$ corresponds to an $n$-tuple of points $\mathbf{x}=\left\{x_{j} \in \Sigma_{A_{j}} \cap \Sigma_{B_{\sigma(j)}}\right\}_{j=1}^{n}$, where $\sigma \in S_{n}$ is a permutation. Further, if $\Sigma_{A_{j}}^{\prime}$ is a small Hamiltonian perturbation of $\Sigma_{A_{j}}$ then each point in $i\left(\Sigma_{A_{j}}^{\prime} \cap \Sigma_{B_{\ell}}\right)$ is close to an intersection point of $A_{j}$ and $B_{\ell}$. Given $x \in \Sigma_{A_{j}}^{\prime} \cap \Sigma_{B_{\ell}}$ let $i(x)$ denote the corresponding intersection point in $A_{j} \cap B_{\ell}$, and given a generator $\mathbf{x}=\left\{x_{j}\right\}$ let $i_{*}(\mathbf{x})=\sum_{j} i\left(x_{j}\right)$, viewed as a 0 -chain in $\mathbb{C}$. As in the proof of Lemma 7.5, we can further arrange that each intersection point of $A_{j}$ and $B_{\ell}$ of type (i-1) (above) corresponds to a single intersection point of $\Sigma_{A_{j}}^{\prime}$ and $\Sigma_{B_{\ell}}$, while each intersection point of type (i-2) corresponds to $2^{m+1}$ intersection points of $\Sigma_{A_{j}}^{\prime}$ and $\Sigma_{B_{\ell}}$.

For the differential, note that a complex structure $j$ on $S$ induces a complex structure $\operatorname{Hilb}^{n}(j)$ on $\operatorname{Hilb}^{n}(S)$. It is possible to achieve transversality for holomorphic curves in $\operatorname{Hilb}^{n}(S)$ using [0,1]-families of complex structures of the form $\operatorname{Hilb}^{n}(j)$, and we will suppose we are working with such complex structures. Then, there is a tautological correspondence between holomorphic disks

$$
\phi:(\mathbb{R} \times[0,1], \mathbb{R} \times\{0\}, \mathbb{R} \times\{1\}) \rightarrow\left(\operatorname{Hilb}^{n}(S), \mathcal{K}_{A}^{\prime}, \mathcal{K}_{B}\right)
$$

connecting $\mathbf{x}$ to $\mathbf{y}$ and holomorphic maps

$$
\psi:(X, \partial X) \rightarrow\left(\mathbb{R} \times[0,1] \times S,\left(\mathbb{R} \times\{0\} \times\left(\Sigma_{A_{1}}^{\prime} \cup \cdots \cup \Sigma_{A_{n}}^{\prime}\right)\right) \cup\left(\mathbb{R} \times\{1\} \times\left(\Sigma_{B_{1}} \cup \cdots \cup \Sigma_{B_{n}}\right)\right)\right)
$$

where $X$ is a Riemann surface with boundary and $2 n$ boundary punctures, $\psi$ is asymptotic to $\{-\infty\} \times[0,1] \times \mathbf{x}$ and $\{+\infty\} \times[0,1] \times \mathbf{y}$, and $\pi_{\mathbb{R} \times[0,1]} \circ \psi$ is an $n$-fold branched covering. The branch points of $\pi_{\mathbb{R} \times[0,1]} \circ \psi$ correspond to $\phi^{-1}(\Delta)$. Given a holomorphic disk in $\operatorname{Hilb}^{n}(S)$ there is an induced homology class $D(\phi)=\left(\pi_{S} \circ \psi\right)_{*}([X, \partial X])$ in $H_{2}\left(S, \Sigma_{A_{1}}^{\prime} \cup \cdots \cup \Sigma_{A_{n}}^{\prime} \cup\right.$ $\left.\Sigma_{B_{1}} \cup \cdots \cup \Sigma_{B_{n}}\right)$ which, inspired by terminology in Heegaard Floer homology, we will call the domain of $\phi$. There is also a projected domain $i_{*} D(\phi) \in H_{2}\left(\mathbb{C}, A_{1} \cup \cdots \cup A_{n} \cup B_{1} \cup \cdots \cup B_{n}\right)$.

If the map $i: S \rightarrow \mathbb{C}$ is holomorphic with respect to an almost complex structure $j_{0}$ on $S$ then all of the coefficients in the projected domain of a $\operatorname{Hilb}^{n}\left(j_{0}\right)$-holomorphic curve are non-negative. It follows that the same holds for almost complex structures sufficiently close to $\operatorname{Hilb}^{n}\left(j_{0}\right)$, and we will assume that we are always working with such almost complex structures.

Note that holomorphic disks which contribute to the symplectic Khovanov differential must lie in $\mathcal{Y}_{n} \subset \operatorname{Hilb}^{n}(S)$, so not every curve of the form (7.9) contributes to the differential. Let $\nabla=\operatorname{Hilb}^{n}(S) \backslash \mathcal{Y}_{n}$. Given a map $\psi$ as in Formula $(7.9)$, let $B(\psi)$ be the preimage in $X$ of the branch points of $\pi_{\mathbb{R} \times[0,1]} \circ \psi$. (The points $B(\psi)$ correspond to the diagonal in $\operatorname{Hilb}^{n}(S)$.) 
Lemma 7.10. For a generic choice of one parameter families of almost complex structures $j$ on $S$, a rigid holomorphic curve $\psi$ as in Formula (7.9) corresponds to a disk in $\mathcal{Y}_{n} \subset \operatorname{Hilb}^{n}(S)$ if and only if

$(Y C) \quad$ The $\operatorname{map}(\mathbb{I} \times i) \circ \psi:(X \backslash B(\psi)) \rightarrow \mathbb{R} \times[0,1] \times \mathbb{C}$ is an embedding. Specifically, this cylindrical formulation $(Y C)$ of maps to $\mathcal{Y}_{n}$ is valid whenever all rigid holomorphic disks in $\left(\operatorname{Hilb}^{n}(S), \mathcal{K}_{A}, \mathcal{K}_{B}\right)$ lie in the complement of $\Delta \cap \nabla$.

Proof. If $a \in \operatorname{Hilb}^{n}(S) \backslash \Delta$, so $a$ corresponds to $n$ distinct points in $S$, then $a \in \mathcal{Y}_{n}$ if and only if $i(a)$ consists of $n$ distinct points in $\mathbb{C}$. Thus, if all rigid holomorphic disks miss $\Delta \cap \nabla$ then the condition of a disk $\phi$ missing $\nabla$ is equivalent to the condition that $i \circ \pi_{S} \circ \psi$ is injective on each regular fiber of $\pi_{\mathbb{R} \times[0,1]} \circ \psi$. This is exactly the extra condition on $\psi$ in the statement of the lemma. As $\Delta \cap \nabla$ is a real codimension 4 subvariety of $\operatorname{Hilb}^{n}(S)$ disjoint from $\mathcal{K}_{A}$ and $\mathcal{K}_{B}$, for a generic choice of almost complex structure all rigid holomorphic disks miss this subspace.

Suppose that a holomorphic curve $\psi$ as in Formula (7.9) satisfies:

- The map $\pi_{\mathbb{R} \times[0,1]} \circ \psi$ has only order 2 branch points and

- If $p, q \in B(\psi)$ are distinct preimages of a branch point then $i\left(\pi_{S}(\psi(p))\right) \neq i\left(\pi_{S}(\psi(q))\right)$.

In this case, the map $\phi: \mathbb{R} \times[0,1] \rightarrow \operatorname{Hilb}^{n}(S)$ corresponding to $\psi$ lies the complement of $\Delta \cap \nabla$. Thus, we can verify that the cylindrical formulation (YC) of maps to $\mathcal{Y}_{n}$ is valid without referencing the Hilbert scheme: we need only check the two conditions above for all rigid holomorphic curves $\psi$.

7.2. A brief review of cyclic and dihedral groups. Some good references for this section are [FP78, Chapter VI, Section 3] and [AM04, Chapter IV, Section 2].

We start by recalling the cohomology of $\mathbb{Z} / 2^{m}=\left\langle\sigma \mid \sigma^{2^{m}}\right\rangle$, with coefficients in $\mathbb{F}_{2}$. The group cohomology is:

$$
H^{*}\left(\mathbb{Z} / 2^{m}\right)= \begin{cases}\mathbb{F}_{2}\left[\alpha, w_{2}\right] /\left(\alpha^{2}+w_{2}\right)=\mathbb{F}_{2}[\alpha] & \text { if } m=1, \\ \mathbb{F}_{2}\left[\alpha, w_{2}\right] /\left(\alpha^{2}\right) & \text { if } m>1 ;\end{cases}
$$

see, for example, [Hat02, Example 3.41]. The reason for the notation $w_{2}$ is that, under the inclusion $\mathbb{Z} / 2^{m} \hookrightarrow S O(2), w_{2}$ is the pullback of the universal second Stiefel-Whitney class in $H^{2}\left(B S O(2) ; \mathbb{F}_{2}\right)$. In the case $m=1$, the class $\alpha$ was denoted $\theta$ earlier in the paper; we have changed notation because soon there will be two different $\mathbb{Z} / 2$-actions.

A particularly nice free resolution of $\mathbb{F}_{2}$ over $\mathbb{F}_{2}\left[\mathbb{Z} / 2^{m}\right]$ is given by

$$
\mathbb{F}_{2}\left[\mathbb{Z} / 2^{m}\right] \stackrel{1+\sigma}{\longleftarrow} \mathbb{F}_{2}\left[\mathbb{Z} / 2^{m}\right] \stackrel{(1+\sigma)^{2^{m}}-1}{\longleftarrow} \mathbb{F}_{2}\left[\mathbb{Z} / 2^{m}\right] \stackrel{1+\sigma}{\longleftarrow} \mathbb{F}_{2}\left[\mathbb{Z} / 2^{m}\right] \stackrel{(1+\sigma)^{2^{m}}-1}{\longleftarrow} \cdots .
$$

So, given a chain complex $C_{*}$ over $\mathbb{F}_{2}\left[\mathbb{Z} / 2^{m}\right]$ we can compute the equivariant cohomology of $C_{*}$ via the complex

$$
C^{*} \stackrel{1+\sigma}{\longrightarrow} C^{*} \stackrel{(1+\sigma)^{2^{m}}-1}{\longrightarrow} C^{*} \stackrel{1+\sigma}{\longrightarrow} C^{*} \stackrel{(1+\sigma)^{2^{m}}-1}{\longrightarrow} \cdots .
$$

The group cohomology $H^{*}\left(\mathbb{Z} / 2^{m}\right)$ acts on the above complex as follows: $w_{2}$ shifts the complex two units to the right; and $\alpha$ is the following map

$$
C^{*} \stackrel{1}{\longrightarrow} C^{*} \stackrel{(1+\sigma)^{2^{m}-2}}{\longrightarrow} C^{*} \stackrel{1}{\longrightarrow} C^{*} \stackrel{(1+\sigma)^{2^{m}-2}}{\longrightarrow} \cdots .
$$

(Compare Lemma 7.15.) 
Next, we turn to the dihedral group of order $2^{m+1}, D_{2^{m}}=\left\langle\sigma, \tau \mid \sigma^{2^{m}}, \tau^{2}, \sigma \tau \sigma \tau\right\rangle$, which acts on $\mathbb{R}^{2}$ and $\mathbb{C}^{2}$ as

$$
\sigma(u, v)=\left(u \cos \theta_{m}-v \sin \theta_{m}, u \sin \theta_{m}+v \cos \theta_{m}\right) \quad \tau(u, v)=(u,-v),
$$

where $\theta_{m}=2 \pi / 2^{m}$, giving a subgroup inclusion $D_{2^{m}} \hookrightarrow O(2)$. Passing to group cohomologies (with coefficients in $\mathbb{F}_{2}$ ), we get a map $H^{*}\left(D_{2^{m}}\right) \leftarrow \mathbb{F}_{2}\left[w_{1}, w_{2}\right]$, where $w_{1} \in H^{1}(B O(2))$ and $w_{2} \in H^{2}(B O(2))$ are the universal first and second Stiefel-Whitney classes. By an abuse of notation, let $w_{1}$ and $w_{2}$ denote their images in $H^{*}\left(D_{2^{m}}\right)$.

For any finite group $G$ with abelianization $\operatorname{Ab}(G), H^{1}(G)$ is just $\operatorname{Hom}\left(\operatorname{Ab}(G), \mathbb{F}_{2}\right)$. Each dihedral group $D_{2^{m}}$ has abelianization isomorphic to $(\mathbb{Z} / 2)^{2}$, generated by $\sigma$ and $\tau$, and therefore, $H^{1}\left(D_{2^{m}}\right) \cong \mathbb{F}_{2} \oplus \mathbb{F}_{2}$. One of the non-zero elements is $w_{1}$, which with respect to the above identification satisfies

$$
w_{1}(\sigma)=0 \quad w_{1}(\tau)=1 \quad w_{1}(\sigma+\tau)=1 .
$$

Let $\alpha, \alpha+w_{1}$ be the other two non-zero elements of $H^{1}\left(D_{2^{m}}\right)$, labeled so that

$$
\begin{aligned}
& \alpha(\sigma)=1 \\
& \alpha(\tau)=0 \\
& \alpha(\sigma+\tau)=1 \\
& \left(\alpha+w_{1}\right)(\sigma)=1 \\
& \left(\alpha+w_{1}\right)(\tau)=1 \\
& \left(\alpha+w_{1}\right)(\sigma+\tau)=0 \text {. }
\end{aligned}
$$

Then the group cohomology is given by

$$
H^{*}\left(D_{2^{m}}\right)= \begin{cases}\mathbb{F}_{2}\left[\alpha, w_{1}, w_{2}\right] /\left(\alpha\left(\alpha+w_{1}\right)+w_{2}\right)=\mathbb{F}_{2}\left[\alpha, w_{1}\right] & \text { if } m=1 \\ \mathbb{F}_{2}\left[\alpha, w_{1}, w_{2}\right] /\left(\alpha\left(\alpha+w_{1}\right)\right) & \text { if } m>1\end{cases}
$$

compare [FP78, Proposition VI.3.1], [AM04, Theorem IV.2.7].

Fix a bounded-below chain complex $C_{*}$ with a left $D_{2^{m}}$-action. As in Section 2 , given any free resolution $R_{*}$ of $\mathbb{F}_{2}$ over $\mathbb{F}_{2}\left[D_{2^{m}}\right]$, we obtain a free resolution $C_{*} \otimes R_{*}$ of $C_{*}$ over $\mathbb{F}_{2}\left[D_{2^{m}}\right]$, and let $\operatorname{RHom}_{\mathbb{F}_{2}\left[D_{2} m\right]}\left(C_{*}, \mathbb{F}_{2}\right)=\operatorname{Hom}_{\mathbb{F}_{2}\left[D_{2} m\right]}\left(C_{*} \otimes R_{*}, \mathbb{F}_{2}\right)$ and $H_{D_{2} m}^{*}\left(C_{*}\right):=$ $\operatorname{Ext}_{\mathbb{F}_{2}\left[D_{2} m\right]}\left(C_{*}, \mathbb{F}_{2}\right)=H_{*}\left(\operatorname{RHom}_{\mathbb{F}_{2}\left[D_{2} m\right]}\left(C_{*}, \mathbb{F}_{2}\right)\right)$. The following is a reasonably simple free resolution of $\mathbb{F}_{2}$ over $\mathbb{F}_{2}\left[D_{2^{m}}\right]$ :

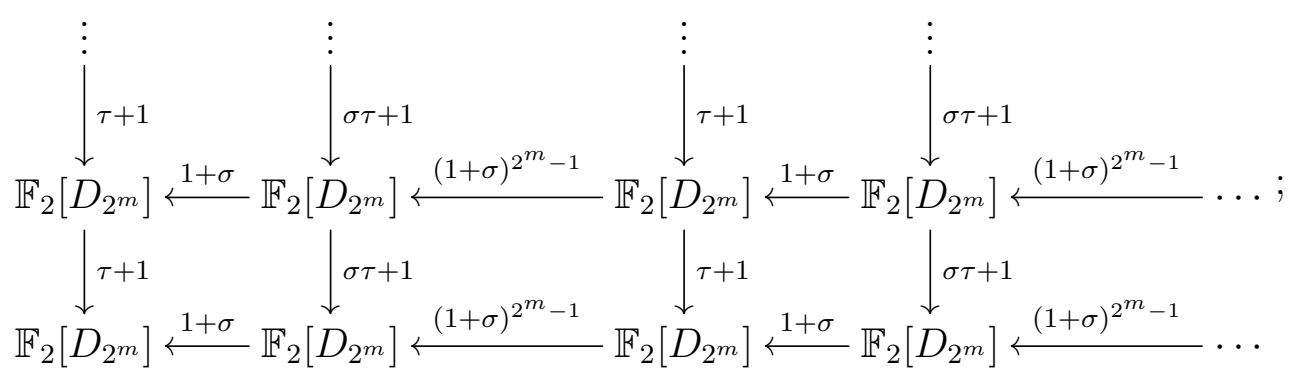

compare [AM04, Proposition IV.2.5]. (This is a diagram of left modules, and the map $\mathbb{F}_{2}\left[D_{2^{m}}\right] \stackrel{x}{\longrightarrow} \mathbb{F}_{2}\left[D_{2^{m}}\right]$ is shorthand for right-multiplication by $x$.) The dual complex $C^{*}$

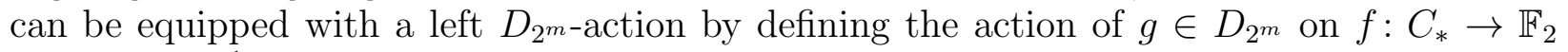
to be $f \circ g^{-1}$. With this left-action, the complex $\operatorname{RHom}_{\mathbb{F}_{2}\left[D_{2} m\right]}\left(C_{*}, \mathbb{F}_{2}\right)$ coming from the 
resolution (7.12) is:

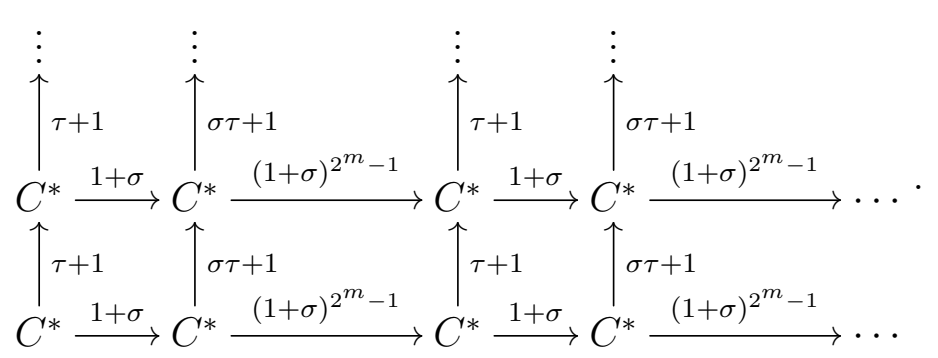

This complex is $\mathbb{Z} \times \mathbb{Z}$-filtered. The associated graded complex with respect to the $\mathbb{Z} \times \mathbb{Z}$ filtration is isomorphic to $C^{*} \otimes H^{*}\left(D_{2^{m}}\right)$. From such a doubly filtered chain complex one can construct many different spectral sequences, each with $E_{1}$-page given by $H^{*}\left(C_{*}\right) \otimes H^{*}\left(D_{2^{m}}\right)$ and $E_{\infty}$-page given by $\operatorname{Ext}_{\mathbb{F}_{2}\left[D_{2} m\right]}\left(C_{*}, \mathbb{F}_{2}\right)$. For instance, consider the set of functions

$$
\left\{f: \mathbb{N} \times \mathbb{N} \rightarrow \mathbb{R} \mid f(a, b)>f\left(a^{\prime}, b^{\prime}\right) \text { if }\left(a \geq a^{\prime}, b \geq b^{\prime},(a, b) \neq\left(a^{\prime}, b^{\prime}\right)\right)\right\} .
$$

Such a function produces an $\mathbb{R}$-filtered complex by declaring the $C^{*}$-summand that lives over the lattice point $(a, b)$ to be in filtration $f(a, b)$. The associated graded object for this singly filtered complex is still $C^{*} \otimes H^{*}\left(D_{2^{m}}\right)$. Restricting to functions $f$ with $\operatorname{Im}(f)=\mathbb{N}$, we get spectral sequences $H^{*}\left(C_{*}\right) \otimes H^{*}\left(D_{2^{m}}\right) \Rightarrow \operatorname{Ext}_{\mathbb{F}_{2}\left[D_{2} m\right]}\left(C_{*}, \mathbb{F}_{2}\right)$.

Lemma 7.15. The action of $H^{*}\left(D_{2^{m}}\right)$ on $H_{D_{2} m}^{*}\left(C_{*}\right)$ is induced from the following actions on the $\mathrm{RHom}_{\mathbb{F}_{2}\left[D_{2} m\right]}\left(C_{*}, \mathbb{F}_{2}\right)$ complex from $(7.13)$ :

- $w_{1}$ shifts the complex one unit up;

- $w_{2}$ shifts the complex two units to the right; and

- $\alpha$ acts as follows (we have suppressed the differentials on the chain complex)

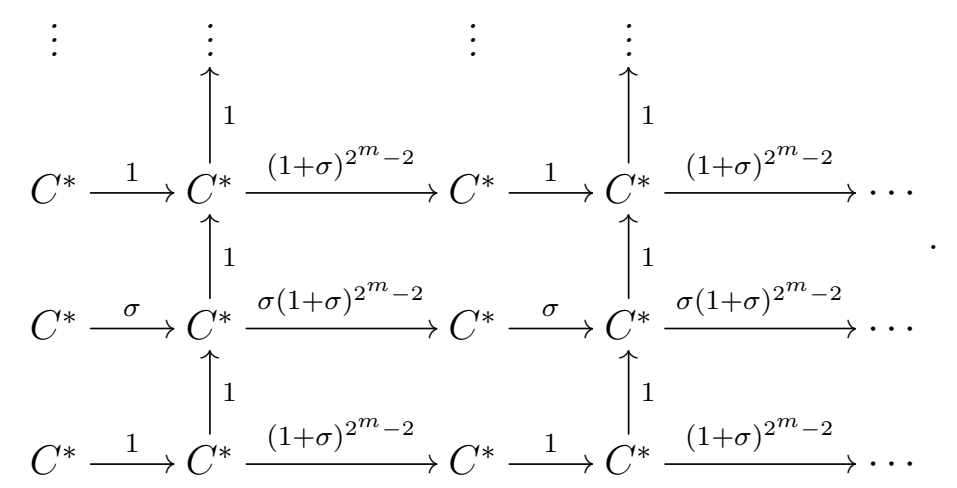

Proof. It is straightforward to check that these actions are chain maps. The actions by $w_{1}$ and $\alpha$ commute up to homotopy, and the action by $\alpha\left(\alpha+w_{1}\right)$ is null-homotopic (respectively, homotopic to the action by $w_{2}$ ) if $m>1$ (respectively, $m=1$ ). Furthermore, these actions are natural with respect to maps induced by $D_{2^{m}}$-equivariant chain maps $C_{*} \rightarrow C_{*}^{\prime}$. One can check by direct computation that these actions induce the ring structure on $H^{*}\left(D_{2^{m}}\right)$ when $C_{*}$ is one-dimensional, and therefore by naturality, these induce the module structure of $H_{D_{2} m}^{*}\left(C_{*}\right)$ over $H^{*}\left(D_{2^{m}}\right)$ for general $C_{*}$.

In particular, by ignoring the $\alpha$-action, the $\operatorname{RHom}_{\mathbb{F}_{2}\left[D_{2} m\right]}\left(C_{*}, \mathbb{F}_{2}\right)$ complex from (7.13) can be viewed as a strict dg-module over $\mathbb{F}_{2}\left[w_{1}, w_{2}\right]$. This also produces a double filtration on the RHom complex by the $w_{1}$ and $w_{2}$ powers. However, this double filtration is 'twice' as coarse as as the previous one (and consequently, carries 'half' the information). 
7.3. A localization result. Let $L$ be a link with $|L|$ components. The $D_{2^{m} \text {-action on }}$ $\left(\mathcal{Y}_{n}, \mathcal{K}_{A}^{\prime}, \mathcal{K}_{B}\right)$ restricts to a $\mathbb{Z} / 2^{m}$-action, for which we have the following localization result:

Theorem 7.16. For each positive integer $m$, the localized equivariant Floer cohomology $w_{2}^{-1} H F_{\mathbb{Z} / 2^{m}}\left(\mathcal{K}_{A}^{\prime}, \mathcal{K}_{B}\right)$ is isomorphic to a direct sum of $2^{|L|}$ copies of $w_{2}^{-1} H^{*}\left(\mathbb{Z} / 2^{m}\right)$. In particular, for $m=1$, we have that $\alpha^{-1} H F_{\mathbb{Z} / 2}\left(\mathcal{K}_{A}^{\prime}, \mathcal{K}_{B}\right)$ is isomorphic to a direct sum of $2^{|L|}$ copies of $\mathbb{F}_{2}\left[\alpha, \alpha^{-1}\right]$.

The case $m=1$ follows easily from the Seidel-Smith localization theorem [SS10, Theorem 20]; see also Proposition 6.3. The general case seems to need an additional argument.

We start by discussing the fixed points of the action. The action of $\mathbb{Z} / 2^{m}$ on $\mathcal{K}_{A}^{\prime} \cap \mathcal{K}_{B}$ is semi-free: each $\mathbb{Z} / 2^{m}$-orbit is either free or trivial. Further, there are exactly $2^{|L|}$ fixed points of the $\mathbb{Z} / 2^{m}$ action: in the notation of Lemma 7.5 the fixed points are $n$-tuples of intersections of type (i-1). (See also Lemma 6.1.) We will refer to these fixed points as $\mathbb{Z} / 2^{m}$-fixed generators.

Lemma 7.17. For any link $L$, there is a bridge diagram $\left(\left\{p_{i}\right\},\left\{A_{i}\right\},\left\{B_{i}\right\}\right)$ so that the $\mathbb{Z} / 2^{m}$ fixed generators are the unique generators of maximal homological grading. In particular, the $\mathbb{Z} / 2^{m}$-fixed generators span a subcomplex of the symplectic Khovanov cochain complex $C F^{*}\left(\mathcal{K}_{A}^{\prime}, \mathcal{K}_{B}\right)$.

Proof. Manolescu explains how to compute the homological grading on $C F\left(\mathcal{K}_{A}^{\prime}, \mathcal{K}_{B}\right)$ in the non-equivariant setting, where each type (i-2) intersection corresponds to two intersections of $\Sigma_{A}^{\prime}$ and $\Sigma_{B}$ [Man06, Section 6.2]. To compute the relative homological grading, first isotope the bridge diagram so that all of the $A_{i}$-arcs are horizontal, and then replace each $B_{i}$ in the bridge diagram by a figure 8 (immersed circle with one transverse double point) $\gamma_{i}$ near $B_{i}$, so that $\gamma_{i}$ intersects each $A_{j}$ at right angles. The generators of $C F\left(\mathcal{K}_{A}^{\prime}, \mathcal{K}_{B}\right)$ correspond to the intersections of $A_{i}$ with $\gamma_{i}$. Given two generators $\mathbf{x}=\left\{x_{i}\right\}$ and $\mathbf{y}=\left\{y_{i}\right\}$, choose a smooth path $\eta_{i}$ in $\gamma_{i}$ from $\mathbf{x} \cap \gamma_{i}$ to $\mathbf{y} \cap \gamma_{i}$, and let $\mu\left(\eta_{i}\right)$ denote the Maslov index of the loop of subspaces $T \eta_{i} \subset \mathbb{R}^{2}$. Then the grading difference between $\mathbf{x}$ and $\mathbf{y}$ is $\sum_{i} \mu\left(\eta_{i}\right)$.

This description carries over to a description of the gradings in the equivariant case, as follows. In the equivariant case, each type (i-2) intersection corresponds to $2^{m}$ intersections of $\Sigma_{A}^{\prime}$ and $\Sigma_{B}$, corresponding to the critical points of a $D_{2^{m}}$-equivariant Morse function on $S^{1}$. Like the indices of the critical points of a Morse function on $S^{1}$, these $2^{m}$ intersection points lie in two adjacent gradings - the same two gradings as the two intersections for the non-equivariant perturbation.

Now, to achieve the conditions of the lemma, wind the $B$-arcs as in Figure 12 . That is, fix a small loop $\zeta_{i}$ around each $p_{i}$ and perform $N_{i} \gg 0$ Dehn twists on the $B$-arcs around each $\zeta_{i}$. From Manolescu's description of the gradings, it is clearly possible to choose the $N_{i}$ so that all of the type (i-1) intersection points have the same grading, and, if the $N_{i}$ are large, this grading is (strictly) higher than the grading of any type (i-2) intersection point. The result follows.

From Lemma 7.17, the localization result follows from homological algebra, which we abstract as its own lemma:

Lemma 7.18. Suppose that $C_{*}$ is a chain complex over $\mathbb{F}_{2}$, with $C_{n}=\mathbb{F}_{2}\left\langle X_{n}\right\rangle$ freely generated by a finite set $X_{n}$, with $X_{n}=\varnothing$ for $|n|$ sufficiently large. Suppose further that $\mathbb{Z} / 2^{m}$ acts on each $X_{n}$, and that this action induces an action by chain maps on $C_{*}$; that the action on each $X_{n}$ is semi-free, so $X_{n}=X_{n}^{\sigma} \amalg X_{n}^{\text {free }}$ where $\sigma$ acts freely on $X_{n}^{\text {free }}$ and fixes $X_{n}^{\sigma}$; and 


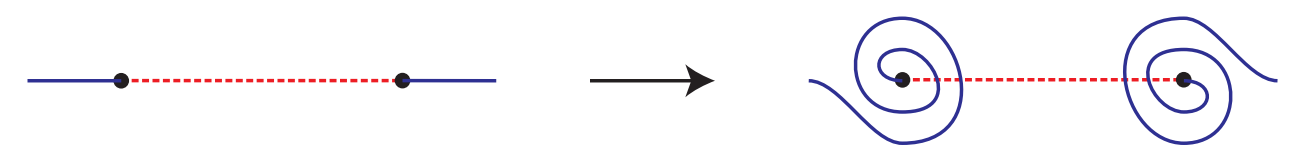

FIgURE 12. Winding a bridge diagram. Perform $N$ Reidemeister I moves near each endpoint, as shown, by winding the $B$-curve clockwise $N$ full turns. The case $N=2$ is shown.

that the $X_{n}^{\text {free }}$ generate a subcomplex $C_{*}^{\text {free }}$ of $C_{*}$. In particular, the dual basis to $X_{n}^{\sigma}$ generates a subcomplex $C_{\sigma}^{*}$ of the dual complex $C^{*}=\operatorname{Hom}_{\mathbb{F}_{2}}\left(C_{*}, \mathbb{F}_{2}\right)$. Let $H\left(C_{\sigma}^{*}\right)$ denote the homology of $C_{\sigma}^{*}$. Then the localized equivariant cohomology

$$
w_{2}^{-1} H_{\mathbb{Z} / 2^{m}}\left(C_{*}\right)=w_{2}^{-1} \operatorname{Ext}_{\mathbb{F}_{2}\left[\mathbb{Z} / 2^{m}\right]}\left(C_{*}, \mathbb{F}_{2}\right)
$$

is isomorphic to $w_{2}^{-1} H^{*}\left(\mathbb{Z} / 2^{m}\right) \otimes_{\mathbb{F}_{2}} H\left(C_{\sigma}^{*}\right)$. In particular, if $m=1$ then

$$
\alpha^{-1} H_{\mathbb{Z} / 2}\left(C_{*}\right)=\alpha^{-1} \operatorname{Ext}_{\mathbb{F}_{2}[\mathbb{Z} / 2]}\left(C_{*}, \mathbb{F}_{2}\right)
$$

is isomorphic to $\alpha^{-1} H^{*}(\mathbb{Z} / 2) \otimes_{\mathbb{F}_{2}} H\left(C_{\sigma}^{*}\right)=\mathbb{F}_{2}\left[\alpha, \alpha^{-1}\right] \otimes_{\mathbb{F}_{2}} H\left(C_{\sigma}^{*}\right)$.

Proof. We can compute the localized equivariant cohomology using the complex

$$
\cdots \stackrel{(1+\sigma)^{2^{m}-1}}{\longrightarrow} C^{*} \stackrel{1+\sigma}{\longrightarrow} C^{*} \stackrel{(1+\sigma)^{2^{m}-1}}{\longrightarrow} C^{*} \stackrel{1+\sigma}{\longrightarrow} C^{*} \stackrel{(1+\sigma)^{2^{m}-1}}{\longrightarrow} \cdots .
$$

where $C^{*}$ denotes the dual complex, over $\mathbb{F}_{2}$, to $C_{*}$.

Inclusion of $C_{\sigma}^{*}$ gives a map $\iota$ of bicomplexes from

$$
\cdots \stackrel{0}{\longrightarrow} C_{\sigma}^{*} \stackrel{0}{\longrightarrow} C_{\sigma}^{*} \stackrel{0}{\longrightarrow} C_{\sigma}^{*} \stackrel{0}{\longrightarrow} C_{\sigma}^{*} \stackrel{0}{\longrightarrow} \cdots .
$$

to (7.19). If we filter both bicomplexes (7.19) and (7.20) by the degree $*$ at each vertex then $\iota$ induces an isomorphism on the $E^{1}$-page of the resulting spectral sequences. Hence, by spectral sequence comparison [McC01, Corollary 3.15], the map $\iota$ is a quasi-isomorphism between the complexes (7.19) and (7.20). The result follows.

Proof of Theorem 7.16. The special case for $m=1$ is immediate from Seidel-Smith's localization theorem [SS10, Theorem 20]. For the general case, since there are no nontrivial disks contained in the fixed set of $\sigma$, we can achieve transversality using a $\mathbb{Z} / 2^{m}$-equivariant almost complex structure $J$ (compare Section 4). Hence, by Proposition 4.5, we may compute the equivariant symplectic Khovanov cohomology using $C F\left(\mathcal{K}_{A}^{\prime}, \mathcal{K}_{B} ; J\right)$ as the chain complex over $\mathbb{F}_{2}\left[\mathbb{Z} / 2^{m}\right]$. By Lemma 7.17 , we can arrange that the $\mathbb{Z} / 2^{m}$-fixed generators span a subcomplex of $C F^{*}\left(\mathcal{K}_{A}^{\prime}, \mathcal{K}_{B} ; J\right)$. Then, via Proposition 7.7 , the hypotheses of Lemma 7.18 are satisfied. There are exactly $2^{|L|} \mathbb{Z} / 2^{m}$-fixed generators, all in the same homological grading, so by Lemma 7.18 the localized equivariant Floer cohomology is a direct sum of $2^{|L|}$ copies of $w_{2}^{-1} H^{*}\left(\mathbb{Z} / 2^{m}\right)$, as desired.

7.4. Equivariant invariance. We start by outlining the non-equivariant proof of invariance of symplectic Khovanov homology. Invariance was originally proved by Seidel-Smith in the case of braid closures [SS06, Theorem 1]; invariance for general bridge diagrams was proved by Waldron [Wal09, Theorem 4.12]. We will mostly follow their arguments.

The first, easy step is independence of the choice of perturbation $\mathcal{K}_{A}^{\prime}$ and of the almost complex structure used to define the Floer homology groups. Both of these statements 


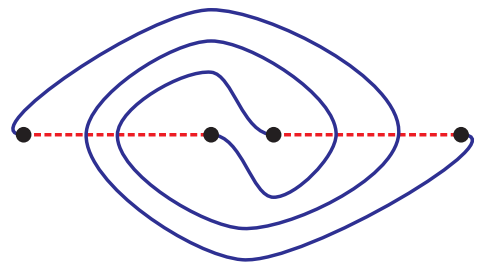

(a)

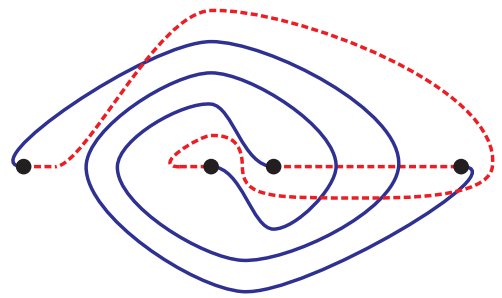

(c)

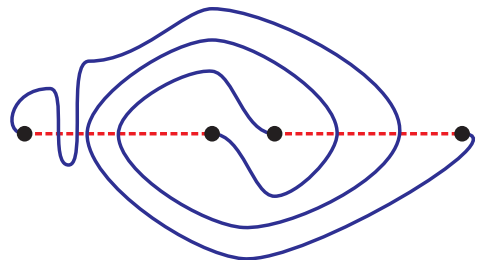

(b)

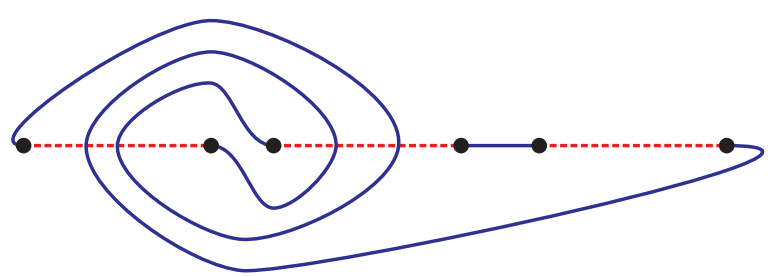

(d)

Figure 13. Moves for bridge diagrams. (a) A bridge diagram. The $A_{i}$ are red and dashed and the $B_{i}$ are blue and solid. (b) The result of an isotopy of the $B_{i}$. (c) The result of a handleslide (or passing move). (d) The result of a stabilization.

follow from the usual continuation map arguments; in the equivariant case, they follow from Proposition 3.28. To keep notation simple, we will suppress the perturbations $\mathcal{K}_{A}^{\prime}$ from the notation in the rest of this section; instances of $\mathcal{K}_{A}$ really mean $\mathcal{K}_{A}^{\prime}$ for some choice of perturbation as in Lemma 7.5.

The meat of the argument, then, is independence of the bridge diagrams. Any two bridge diagrams for the same link can be connected by a sequence of:

(B1) Isotopies of the $\operatorname{arcs} A_{i}$ (or rather, $\cup_{i} A_{i}$ ) and the $\operatorname{arcs} B_{i}$ (or rather, $\cup_{i} B_{i}$ ), rel endpoints.

(B2) Handleslides ("passing moves" in Waldron's terminology).

(B3) Stabilizations.

Note that move (B1) induces both Reidemeister I and II moves of the knot diagram, and move (B2) induces Reidemeister III moves. The bridge moves are illustrated in Figure 13.

The proof of invariance has two steps:

(1) Isotopies and handleslides induce Hamiltonian isotopies of $\mathcal{K}_{A}$ and $\mathcal{K}_{B}$. This is obvious for isotopies, from either Seidel-Smith's or Manolescu's formulation. For handleslides, see Seidel-Smith [SS06, Lemma 48].

(2) Stabilizations. This is proved by a degeneration argument [SS06, Section 5.4]; the rest of this section gives a slightly different degeneration proof of stabilization invariance.

7.4.1. Non-equivariant stabilization invariance. Because of handleslide invariance, it suffices to prove stabilization invariance for a stabilization at a point $p \in B_{n}$ adjacent to $\infty$, i.e., introducing a new arc $A_{n+1}^{\prime}$ adjacent to the unbounded region of the knot complement, and replacing $B_{n}$ by $B_{n}^{\prime}$ and $B_{n+1}^{\prime}$. Let $S$ and $S^{\prime}$ denote the surface (7.1) before and after the stabilization, respectively. Let $R$ denote the bounded region in the stabilized diagram $\left(\mathbb{C}, A_{1} \cup \cdots \cup B_{n+1}^{\prime}\right)$ adjacent to $A_{n+1}^{\prime}$, and let $R_{0}$ denote the bounded region in unstabilized 
diagram $\left(\mathbb{C}, A_{1} \cup \cdots \cup B_{n}\right)$ adjacent to $p$. Let $\left\{p_{2 n+1}, p_{2 n+2}\right\}=A_{n+1}^{\prime} \cap\left(B_{n}^{\prime} \cup B_{n+1}^{\prime}\right)$, so that the arc $A_{n+1}^{\prime}$, oriented as part of the boundary of $R$, runs from $p_{2 n+1}$ to $p_{2 n+2}$.

The correspondence between generators of the Floer complexes before and after the stabilization is fairly clear. Each of $\Sigma_{A_{n+1}^{\prime}} \cap \Sigma_{B_{n+1}^{\prime}}$ and $\Sigma_{A_{n+1}^{\prime}} \cap \Sigma_{B_{n}^{\prime}}$ consists of a single point, and any generator $\mathbf{x}$ for the stabilized diagram contains exactly one of these two points, as well as one point from $\left(\Sigma_{B_{n}^{\prime}} \cup \Sigma_{B_{n+1}^{\prime}}\right) \cap\left(\Sigma_{A_{1}} \cup \cdots \cup \Sigma_{A_{n}}\right)$ and $(n-1)$ other points in $\left(\Sigma_{B_{1}} \cup \cdots \cup \Sigma_{B_{n-1}}\right) \cap\left(\Sigma_{A_{1}} \cup \cdots \cup \Sigma_{A_{n}}\right)$. The points of $\left(\Sigma_{B_{n}^{\prime}} \cup \Sigma_{B_{n+1}^{\prime}}\right) \cap\left(\Sigma_{A_{1}} \cup \cdots \cup \Sigma_{A_{n}}\right)$ for the stabilized diagram correspond to the points of $\Sigma_{B_{n}} \cap\left(\Sigma_{A_{1}} \cup \cdots \cup \Sigma_{A_{n}}\right)$; in Figure 14, this corresponds to the fact that $B_{n}^{\prime} \cup B_{n+1}^{\prime}$ agrees with $B_{n}$ on one side of the line $C$. Forgetting the point on $\left(\Sigma_{B_{n}^{\prime}} \cup \Sigma_{B_{n+1}^{\prime}}\right) \cap \Sigma_{A_{n+1}^{\prime}}$, using the correspondence

$$
\left[\left(\Sigma_{B_{n+1}^{\prime}} \cup \Sigma_{B_{n}^{\prime}}\right) \cap\left(\Sigma_{A_{1}} \cup \cdots \cup \Sigma_{A_{n}}\right)\right] \cong\left[\Sigma_{B_{n}} \cap\left(\Sigma_{A_{1}} \cup \cdots \cup \Sigma_{A_{n}}\right)\right]
$$

and leaving the other $(n-1)$ points alone gives the corresponding generator for the unstabilized diagram.

Before studying the holomorphic disks, we make an observation about their domains:

Lemma 7.21. The projected domain of any holomorphic disk in $\left(\mathcal{Y}_{n}, \mathcal{K}_{A}, \mathcal{K}_{B}\right)$, respectively $\left(\mathcal{Y}_{n+1}, \mathcal{K}_{A^{\prime}}, \mathcal{K}_{B^{\prime}}\right)$, has multiplicity 0 or 1 at $R_{0}$, respectively $R$.

Proof. Our conditions on the complex structures imply that the projected domain of any holomorphic disk has only non-negative coefficients (compare [OSz04b, Lemma 3.2]). Further, given generators $\mathbf{x}$ and $\mathbf{y}$ of the Floer complex, the boundary of any projected domain $i_{*} D(\phi)$ of a curve from $\mathbf{x}$ to $\mathbf{y}$ satisfies $\partial\left(\left(\partial i_{*} D(\phi)\right) \cap A\right)=i_{*}(\mathbf{y})-i_{*}(\mathbf{x})$, as 0-chains in $\mathbb{C}$. Thus, the coefficients of the projected domain at two adjacent regions (separated by $A_{i}$ or $B_{i}$ ) can differ by at most one. Since the coefficient of the projected domain at the unbounded region is zero, the lemma follows. (For instance, in the stabilized diagram, $i_{*} D(\phi)$ has multiplicity 0 at $R$ if and only if $\mathbf{x} \cap\left\{p_{2 n+1}, p_{2 n+2}\right\}=\mathbf{y} \cap\left\{p_{2 n+1}, p_{2 n+2}\right\}, i_{*} D(\phi)$ has multiplicity 1 at $R$ if and only if $p_{2 n+1} \in \mathbf{x}$ and $p_{2 n+2} \in \mathbf{y}$, and $i_{*} D(\phi)$ cannot have multiplicity 2 or higher at $R$.)

We turn now to the identification of the differentials on the stabilized and un-stabilized diagrams, using a degeneration argument. This argument will be given in the cylindrical formulation of Section 7.1.3, and is similar to (a simple case of) the neck stretching argument underlying bordered Heegaard Floer homology [LOT08, Section 9.1].

Consider a vertical line $C$ which separates $A_{n+1}^{\prime}$ from $A_{1} \cup \cdots \cup A_{n}$, intersects $B_{n}^{\prime}$ and $B_{n+1}^{\prime}$ in one point each, and is disjoint from $B_{1} \cup \cdots \cup B_{n-1} \cup A_{1} \cup \cdots \cup A_{n} \cup A_{n+1}^{\prime}$. (See Figure 14.) Assume that $B_{n}^{\prime}$ and $B_{n+1}^{\prime}$ are horizontal near $C$. Let $E$ denote the component of $\mathbb{C} \backslash C$ containing $A_{1}, \ldots, A_{n}$ and let $D$ denote the component of $\mathbb{C} \backslash C$ containing $A_{n+1}^{\prime}$. Choose a family of complex structures $j_{T}^{\mathbb{C}}$ on $\mathbb{C}$ for $T$ in $\mathbb{R}$ large with long necks along $C$, so that the length of the neck tends to infinity as $T \rightarrow \infty$, and almost complex structures $j_{T}^{\prime}$ on $S^{\prime}$ so that the projection $i: S^{\prime} \rightarrow \mathbb{C}$ is $\left(j_{T}^{\prime}, j_{T}^{\mathbb{C}}\right)$-holomorphic and $j_{T}^{\prime}$ is induced from $j_{T}^{\mathbb{C}}$ via Formula (7.1) over $D$. (As usual, by almost complex structure we mean a [0,1]-parameter family of almost complex structures, though we assume the family is constant over $D$. For readability, we will continue to suppress the $[0,1]$ parameter from the discussion.) There are induced almost complex structures $\operatorname{Hilb}^{n+1}\left(j_{T}^{\prime}\right)$ on $\operatorname{Hilb}^{n+1}\left(S^{\prime}\right)$.

Let $\sigma_{n}^{\prime}=\Sigma_{B_{n}^{\prime}} \cap i^{-1}(C)$ and $\sigma_{n+1}^{\prime}=\Sigma_{B_{n+1}^{\prime}} \cap i^{-1}(C)$, so $\sigma_{n}^{\prime}$ and $\sigma_{n+1}^{\prime}$ are topologically circles. There is a vector field $\vec{R}$ on $i^{-1}(C)$ corresponding to the stretching, which is a lift of the 

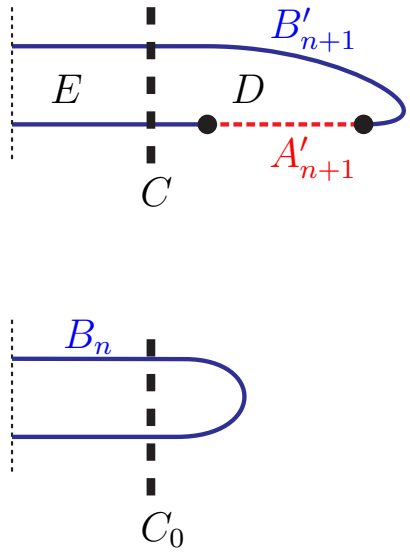
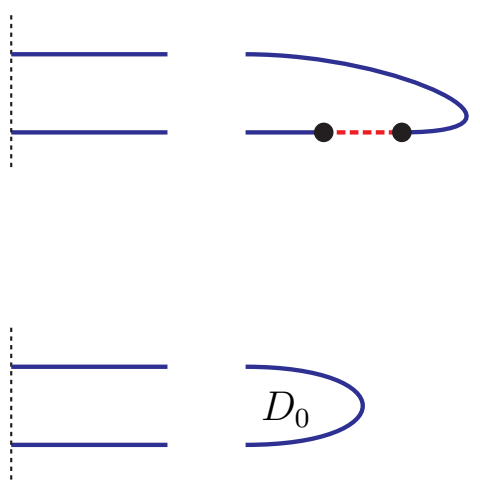

FIGURE 14. Stabilization invariance for symplectic Khovanov homology. In all cases, only the stabilization region is shown; the rest of the knot is to the left.

vector field $\frac{\partial}{\partial y}$ on $C$ to $S^{\prime}$. The data $\left(i^{-1}(C), \vec{R}\right)$ is adjusted to the Kähler form on $S^{\prime}$ in the sense of $\left[\mathrm{BEH}^{+} 03\right.$, Section 3.4]. For each point $q \in \sigma_{n}^{\prime}$ there is a unique $\vec{R}$-chord $\gamma_{q}$ starting at $q$ and ending on $\sigma_{n+1}^{\prime}$. (In particular, the flow of $\vec{R}$ is Morse-Bott with respect to $\sigma_{n}^{\prime}$ and $\sigma_{n+1}^{\prime}$.) In fact, these chords lie in the surfaces $i^{-1}(C) \cap\{v / u=$ constant $\}$ (which are Heegaard surfaces for the branched double cover of $L$ [Man06, Section 7.3]; see Figure 15).

Lemma 7.22. For generic $j_{T}^{\mathbb{C}}$ and $j_{T}^{\prime}$ as above, the rigid moduli spaces of $\operatorname{Hilb}^{n+1}\left(j_{T}^{\prime}\right)$ holomorphic disks, or equivalently the moduli spaces of maps as in Equation (7.9), are transversely cut out for all sufficiently large $T$.

Proof. Given a non-constant holomorphic disk $u$ in $\mathcal{Y}_{n+1}$ we can find a point $p \in \mathbb{D}^{2}$ so that $i(u(p)) \in \operatorname{Hilb}^{n+1}(\mathbb{C})$ consists of $n+1$ distinct points. Writing $u(p)=\left\{q_{1}, \ldots, q_{n+1}\right\}$ and $i(u(p))=\left\{z_{1}, \ldots, z_{n+1}\right\}$, the tangent space at $u(p)$ to $\operatorname{Hilb}^{n+1}\left(S^{\prime}\right)$ decomposes as

$$
T_{u(p)}\left(\operatorname{Hilb}^{n+1}\left(S^{\prime}\right)\right)=\left(T_{q_{1}} i^{-1}\left(z_{1}\right)\right) \oplus\left(T_{z_{1}} \mathbb{C}\right) \oplus \cdots \oplus\left(T_{q_{n+1}} i^{-1}\left(z_{n+1}\right)\right) \oplus\left(T_{z_{n+1}} \mathbb{C}\right) .
$$

Our conditions on the almost complex structures amount to choosing an almost complex structure on each summand separately. This is enough flexibility for standard transversality arguments (see, e.g., [MS04, Section 3.2]) to go through.

By Lemma 7.21, there are two cases: projected domains with multiplicity 0 at $D \cap R$ and projected domains with multiplicity 1 at $D \cap R$. The first case is simple: it is analogous to the stabilization invariance proof for the Heegaard Floer homology group $\widehat{H F}$ [OSz04b, Section 10]. So, we will focus on the case of projected domains with multiplicity 1 at $D \cap R$.

As the neck stretching parameter $T$ goes to infinity, $\left(j_{[0,1] \times \mathbb{R}} \times j_{T}^{\prime}\right)$-holomorphic maps of the form (7.9) converge to pairs of holomorphic maps

$$
\begin{array}{r}
v_{\infty}^{D}:\left(X_{D}, \partial X_{D}\right) \rightarrow\left(\mathbb{R} \times[0,1] \times i^{-1}(D),\left(\mathbb{R} \times\{0\} \times \Sigma_{A_{n+1}^{\prime}}\right) \cup\left(\mathbb{R} \times\{1\} \times \Sigma_{B_{n}^{D}}\right)\right) \\
v_{\infty}^{E}:\left(X_{E}, \partial X_{E}\right) \rightarrow\left(\mathbb{R} \times[0,1] \times i^{-1}(E),\left(\left(\mathbb{R} \times\{0\} \times\left(\Sigma_{A_{1}} \cup \cdots \cup \Sigma_{A_{n}}\right)\right)\right.\right. \\
\left.\left.\cup\left(\mathbb{R} \times\{1\} \times\left(\Sigma_{B_{1}} \cup \cdots \cup \Sigma_{B_{n-1}} \cup \Sigma_{B_{n}^{E}}\right)\right)\right)\right)
\end{array}
$$

so that 
- $X_{D}$ has three boundary punctures.

- $X_{E}$ has $2 n+1$ boundary punctures.

- There is a boundary puncture $p_{D}$ of $X_{D}$ and $p_{E}$ of $X_{E}$ so that at $p_{D}$ the map $\pi_{S^{\prime}} \circ v_{\infty}^{D}: X_{D} \rightarrow i^{-1}(D)$ is asymptotic to an $\vec{R}$-chord $\gamma$ in $i^{-1}(C)$ between $\sigma_{n}^{\prime}$ and $\sigma_{n+1}^{\prime}$ and at $p_{E}$ the map $\pi_{S^{\prime}} \circ v_{\infty}^{E}: X_{E} \rightarrow i^{-1}(E)$ is asymptotic to the same chord $\gamma$.

- Filling in the puncture $p_{D} \circ v_{\infty}^{D}$, the map $\pi_{\mathbb{R} \times[0,1]} \circ v_{\infty}^{D}: X_{D} \rightarrow \mathbb{R} \times[0,1]$ extends to a degree 1 branched cover (i.e., a diffeomorphism).

- Filling in the puncture $p_{E}$, the map $\pi_{\mathbb{R} \times[0,1]} \circ v_{\infty}^{E}: X_{E} \rightarrow \mathbb{R} \times[0,1]$ extends to a degree $n$ branched cover.

(To deduce the compactness theorem we are using, fix a sequence $v_{T}$ of $j_{T}^{\prime}$-holomorphic curves and consider the two projections $\pi_{S^{\prime}} \circ v_{T}$ and $\pi_{\mathbb{R} \times[0,1]} \circ v_{T}$ separately, along the lines of [LOT08, Section 5.4]. The gluing result follows from standard arguments, along the lines of [Lip06, Proposition A.2], say, which in turn follows arguments from [MS04].)

We claim that for any curve $v_{\infty}^{E}$ as in Formula (7.24) asymptotic to some chord $\gamma$ in $i^{-1}(C)$ there is a unique curve $v_{\infty}^{D}$ as in Formula (7.23) asymptotic to the same chord $\gamma$. Thus, for large $T,\left(j_{[0,1] \times \mathbb{R}} \times j_{T}^{\prime}\right)$-holomorphic curves as in Equation (7.9) correspond to $\left(j_{[0,1] \times \mathbb{R}} \times j_{\infty}^{\prime}\right)$ holomorphic curves $v_{\infty}^{E}$ as in Formula (7.24).

To see this, consider the Riemann surface $\mathcal{H}:=i^{-1}(D) \cap\{u=0\}$ and its intersections with $\Sigma_{A_{n+1}^{\prime}}, \Sigma_{B_{n}^{\prime}}^{D}$, and $\Sigma_{B_{n+1}^{\prime}}^{D}$, as shown in Figure 15. There are two chords between $\Sigma_{B_{n}^{\prime}}^{D} \cap \mathcal{H}$ and $\Sigma_{B_{n+1}^{\prime}}^{D} \cap \mathcal{H}$, labeled $\gamma$ and $\gamma^{\prime}$ in the figure. With respect to the standard complex structure on $S^{\prime}$ induced from Formula (7.1), there is a unique holomorphic disk as in Formula (7.23) asymptotic to $\gamma$ (respectively $\gamma^{\prime}$ ). It follows from boundary injectivity [OSz04b, Proposition $3.9]$ that these disks are transversally cut out in $\mathcal{H}$. Further, the normal Maslov index to these disks is 1, so it follows from automatic transversality [HLS97, Theorem 2] that these holomorphic disks are transversally cut out in $i^{-1}(D)$. Now, the $S^{1}$ action on $S^{\prime}$ (or rather, $i^{-1}(D)$ ) implies that, with respect to the standard complex structure, there are similar disks asymptotic to each chord from $\Sigma_{B_{n}^{\prime}}^{D}$ to $\Sigma_{B_{n+1}^{\prime}}^{D}$.

Next, we claim these are, algebraically, the only non-constant holomorphic curves of the form $v_{\infty}^{D}$. To see that, note that the count of holomorphic curves $v_{\infty}^{E}$ asymptotic to a generic chord $\gamma$ is invariant under Lagrangian isotopies of $B_{n}^{\prime} \cup B_{n+1}^{\prime}$. So, consider instead the diagram in which $D \cap R$ consists of the upper half plane, $D \cap\left(B_{n}^{\prime} \cup A_{n+1}^{\prime} \cup B_{n+1}^{\prime}\right)$ is the real axis, $\partial A_{n+1}^{\prime}=\{0,1\}$, and the polynomial $p(z)$ is simply $p(z)=z(z-1)$. Consider the projection $\pi_{u}$ to the $u$-coordinate. The boundary conditions $\Sigma_{A_{n+1}}, \Sigma_{B_{n}^{\prime}} \cap D$, and $\Sigma_{B_{n+1}^{\prime}} \cap D$ project to $\mathbb{R}, i \mathbb{R}$, and $i \mathbb{R}$ respectively. Because of the $S^{1}$-symmetry, it suffices to consider the case that $\gamma$ lies in the surface $\mathcal{H}$. Fix a map $v_{\infty}^{D}: X_{D} \rightarrow \mathbb{R} \times[0,1] \times i^{-1}(D)$ asymptotic to $\gamma$. The map $i \circ v_{\infty}^{D}$ identifies $X_{D}$ with the upper half plane. With respect to this identification, $\pi_{u} \circ v_{\infty}^{D}$ is a map from the upper half plane to $\mathbb{C}$ sending the boundary to a bounded subset of $\mathbb{R} \cup i \mathbb{R}$ (because $\pi_{u}(\gamma)=0$ ). It follows from the open mapping principle that $\pi_{u} \circ v_{\infty}^{D}$ is constant, so $v_{\infty}^{D}$ is contained in $\mathcal{H}$ and so $v_{\infty}^{D}$ must be one of the curves in the $S^{1}$-family previously constructed. In particular, the count of curves $v_{\infty}^{D}$ asymptotic to $\gamma$ is 1 .

Thus, the count of $j_{T}^{\prime}$-holomorphic disks as in Formula (7.9), for $T$ large, is the same as the count of curves $u_{\infty}^{E}$ as in Formula (7.23), with no restriction on the chord $\gamma_{q}$. By Lemma 7.21, the condition that the curve with large $T$ lies in $\mathcal{Y}_{n+1} \subset \operatorname{Hilb}^{n+1}\left(S^{\prime}\right)$ corresponds to the condition that the limiting curve lies in $\mathcal{Y}_{n} \subset \operatorname{Hilb}^{n}(E)$; see also Lemma 7.10. 


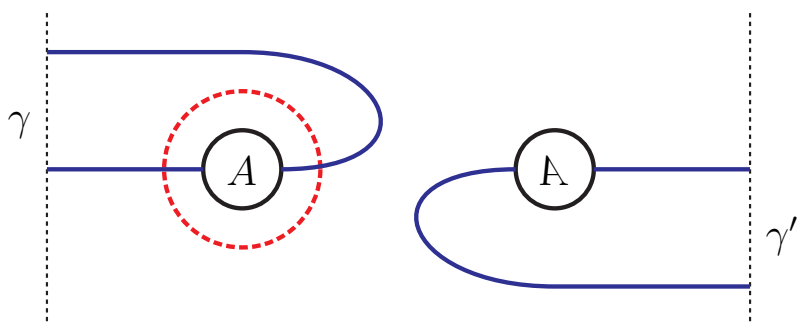

Figure 15. The surface $i^{-1}(D) \cap\{u=0\}$. This is the branched double cover of $D$ along the points $p_{2 n+1}$ and $p_{2 n+2}$. The circles labeled $A$ are connected by a handle; the branch points are at the intersections of the $\Sigma_{A_{n+1}^{\prime}} \cap \mathcal{H}$ (dashed) and $\left(\Sigma_{B_{n}^{\prime}}^{E} \cup \Sigma_{B_{n+1}^{\prime}}^{E}\right) \cap \mathcal{H}$ (solid).

Next, consider instead stretching the neck along a vertical line $C_{0}$ in the un-stabilized diagram $\left(\mathbb{C}, A_{1}, \ldots, A_{n}, B_{1}, \ldots, B_{n}\right)$ which intersects $B_{n}$ perpendicularly in two points and is disjoint from the other arcs. (Again, see Figure 14.) This time, holomorphic curves of the form (7.9) converge to pairs $\left(v_{\infty}^{D}, v_{\infty}^{E}\right)$ where $v_{\infty}^{E}$ is of the form (7.24) and $v_{\infty}^{D}$ is a holomorphic disk with one puncture mapping to $\mathbb{R} \times[0,1] \times i^{-1}\left(D_{0}\right)$ which is constant in the $\mathbb{R} \times[0,1]$-direction, and the map $i \circ \pi_{S} \circ v_{\infty}^{D}$ is a diffeomorphism to $D_{0} \cap R_{0}$. (This is similar to the boundary degenerations in Heegaard Floer theory [OSz08, Section 5].) Again, there is a unique disk $v_{\infty}^{D}$ asymptotic to each chord $\gamma$, and in spite of the somewhat degenerate situation, these curves are transversely cut out by the $\bar{\partial}$-equations. So, for large $T$, holomorphic curves in the un-stabilized diagram $S$ of the form (7.9) correspond to maps $v_{\infty}^{E}$ of the form (7.24), and hence to curves in the stabilized diagram. This completes the cylindrical proof of stabilization invariance.

\subsubsection{Equivariant invariance.}

Proof of Theorem 1.26. As in the non-equivariant case, it suffices to check invariance under isotopies and handleslides of the $A_{j}$ and $B_{j}$ and stabilizations of the diagram.

It is clear that an isotopy of the $A_{j}$ is covered by an $O(2)$-equivariant Lagrangian (and hence Hamiltonian - $H^{1}\left(\mathcal{Y}_{n}\right)$ vanishes) isotopy of the Lagrangians $\mathcal{K}_{A}$ and $\mathcal{K}_{B}$; and this isotopy extends to an isotopy between the $D_{2^{m}}$-equivariant perturbations of $\mathcal{K}_{A}^{\prime}$ from Lemma 7.5. Thus, Proposition 3.28 implies that the quasi-isomorphism type of $C F_{D_{2} m}\left(\mathcal{K}_{A}, \mathcal{K}_{B}\right)$, over $\mathbb{F}_{2}\left[D_{2^{m}}\right]$, is unchanged by isotopies.

As noted earlier handleslides give Lagrangian isotopies in the Hilbert scheme [SS06, Lemma 48]. Rather than checking that these isotopies can be made equivariant, we appeal to Proposition 3.29. For notational simplicity, assume we are sliding $A_{1}$ over $A_{2}$. Let $\mathcal{K}_{A}$ and $\mathcal{K}_{\widetilde{A}}$ be the Lagrangians before and after the handleslide. By Proposition 3.29, to prove handleslide invariance it suffices to verify that we can choose equivariant perturbations $\mathcal{K}_{A}^{\prime}$ and $\mathcal{K}_{\widetilde{A}}^{\prime}$ of $\mathcal{K}_{A}$

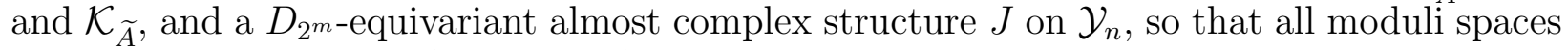
of holomorphic disks in $\left(\mathcal{Y}_{n}, \mathcal{K}_{A}^{\prime}, \mathcal{K}_{\widetilde{A}}^{\prime}\right)$ with Maslov index $\leq 1$ are transversally cut out, and

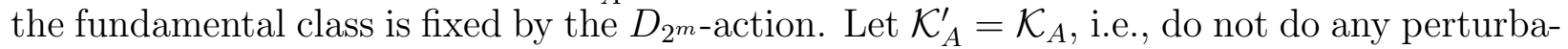
tion at all. For the perturbation $\mathcal{K}_{\widetilde{A}}^{\prime}$ perturb the handleslid arcs $\widetilde{A}$ so that they intersect the original arcs $A$ only at endpoints and let $\mathcal{K}_{\widetilde{A}}^{\prime}$ be the associated Lagrangian. (So, both $\mathcal{K}_{A}^{\prime}$ and 

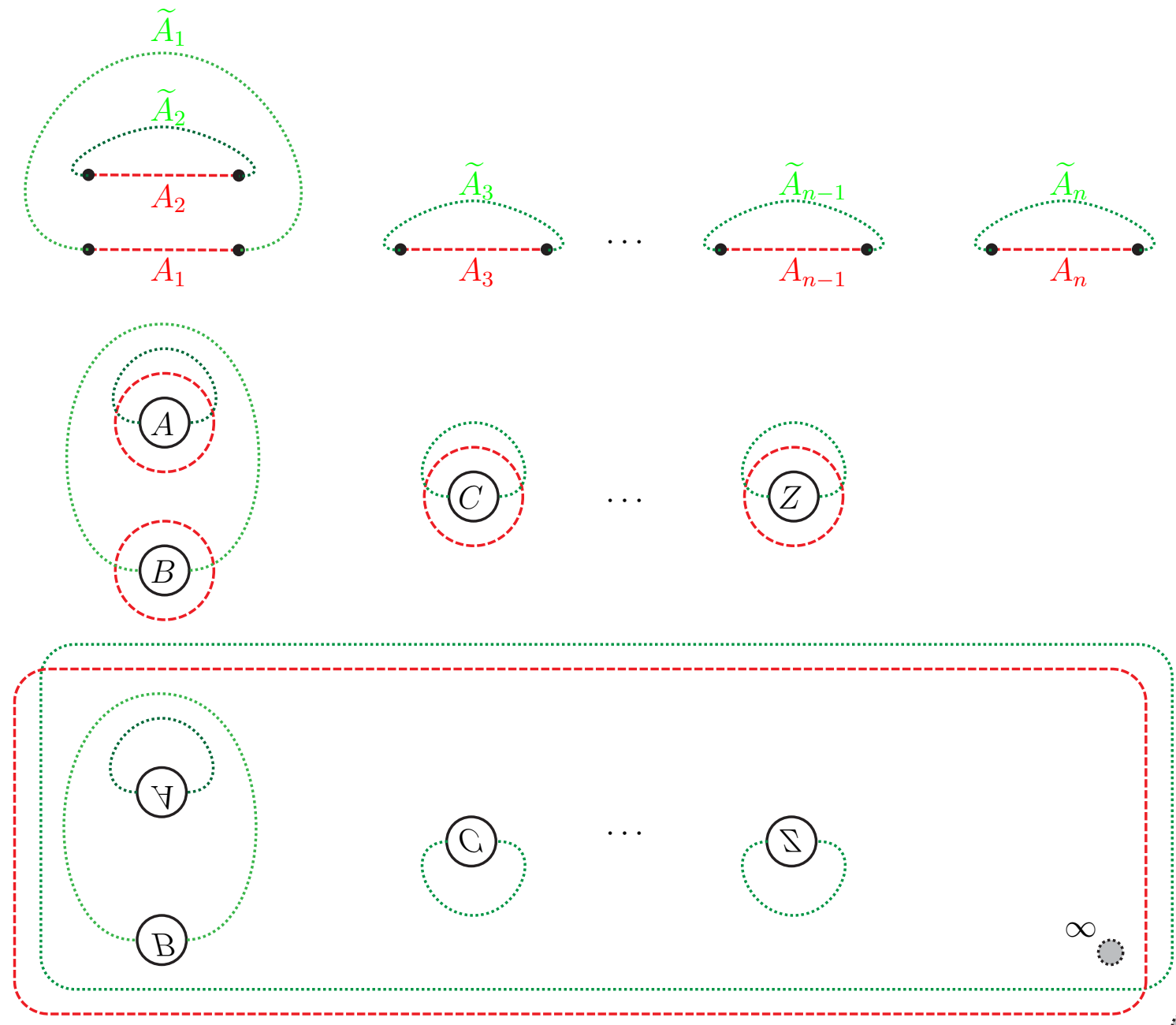

FIGURE 16. Equivariant handleslide invariance. Top: Up to diffeomorphism of $\mathbb{C}$, the $\operatorname{arcs} A$ and $\widetilde{A}$ in the proof of handleslide invariance are as shown. Bottom: the branched double covers of the pieces at the top, branched along the $p_{i}$. The small gray circles indicate the two non-compact regions.

$\mathcal{K}_{\widetilde{A}}^{\prime}$ are $O(2)$-invariant.) See Figure 16. It follows that the Floer complex $C F\left(\mathcal{K}_{\tilde{A}}^{\prime}, \mathcal{K}_{\widetilde{A}}^{\prime}\right)$ has rank $2^{n}$; since the diagram $(A, \widetilde{A})$ represents an $n$-component unlink, $K h_{\text {symp }}(A, \widetilde{A})$ also has rank $2^{n}$, and the differential on $C F\left(\mathcal{K}_{A}^{\prime}, \mathcal{K}_{\widetilde{A}}^{\prime}\right)$ must vanish. (The differential also vanishes for grading reasons: all generators lie in the same grading modulo 2.) Note that all intersection

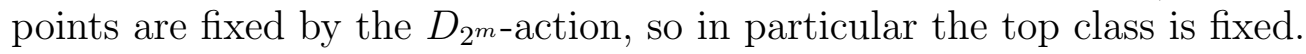

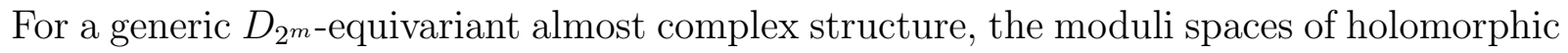
disks not entirely contained in the fixed set of some $g \neq 1 \in D_{2^{m}}$ are transversally cut out; compare [KS02, Section 5c]. The components of the fixed sets of $\sigma^{i}$ intersecting $\mathcal{K}_{A}^{\prime}$ and $\mathcal{K}_{\widetilde{A}}^{\prime}$ are discrete, and hence contain no non-constant holomorphic disks. The fixed set of $\sigma^{i} \tau$ is the symmetric product of a Heegaard diagram for $\Sigma(K)$ with an extra $\alpha$ and $\beta$ circle, as shown in the bottom of Figure 16. (More precisely, the fixed set is the complement of the anti-diagonal inside this symmetric product, since we are considering $\mathcal{Y}_{n}$, not all of $\operatorname{Hilb}^{n}(S)$.) Positivity of domains implies that there are no holomorphic curves in this fixed 
set with negative Maslov index in $\mathcal{Y}_{n}\left(\right.$ or in $\operatorname{Sym}^{n}(\Sigma)$ ). For a generic perturbation of the $A_{i}$ and $\widetilde{A}_{i}$, the index 0 domains in the Heegaard diagram have no holomorphic representatives (by boundary injectivity [OSz04b, Proposition 3.9]), so in particular those moduli spaces are transversally cut out at as well. There are no homotopy classes of disks with index 1. So, the hypotheses of Proposition 3.29 are satisfied, and handleslide invariance follows.

Finally, for stabilization invariance, the proof described in Section 7.4.1 never breaks the $D_{2^{m}}$ symmetry, so the isomorphisms described there (for large $T$ ) give isomorphisms of freed Floer complexes. (Note that, in choosing the families of almost complex structures in the definition of the freed Floer complex, we may assume the families are constant over the region $D$ from Section 7.4.1 and still achieve transversality.)

Proof of Corollary 1.4. This follows from Theorem 1.26; see also the proof of Corollary 1.10.

Proof of Corollary 1.5. The proof is similar to the proof of Theorem 1.26, but now working equivariantly with respect to a symmetry on the bridge diagram, rather than the $O(2)$ symmetry on $S$. Any two periodic bridge diagrams for the same knot and period can be related by a sequence of equivariant isotopies, pairs of handleslides, and pairs of stabilizations: find a sequence of bridge moves relating the two quotient knots and pull back the diagrams. Invariance under equivariant isotopies is immediate from Proposition 3.28. Invariance under handleslides follows from Proposition 3.29 just as in the proof of Theorem 1.26; the fact that we are doing pairs of handleslides at once leads to only notational changes. Equivariant stabilization invariance follows from the argument outlined in Section 7.4.1. Again, the fact that we are performing pairs of stabilizations simultaneously does not lead to additional complications: the neck stretching argument happens in two separate regions of the diagram.

7.5. Invariance of reduced symplectic Khovanov homology. As a last theorem of the paper, we fill a small gap in the literature not related to equivariant Floer homology. As discussed in Section 7.1, Manolescu defined a reduced symplectic Khovanov homology, by deleting one $A_{i}$-arc and one $B_{i}$-arc and working in the space $\widetilde{\mathcal{Y}}_{n}$. It seems that invariance of the reduced symplectic Khovanov homology has not been verified previously.

In this section, we work with coefficients in $\mathbb{Z}$, rather than just $\mathbb{Z} / 2$.

Theorem 7.25. The reduced symplectic Khovanov homology $\widetilde{K h}_{\text {symp }}(K, p)$ is an invariant of the based link $(K, p)$.

Proof. We must check invariance under:

(1) Regular homotopies of the bridge diagram.

(2) Handleslides of the $A_{i}$ and $B_{i}$, where we do not handleslide over the deleted curves $A_{n}$ or $B_{n}$.

(3) Handleslides of an $A_{i}$ across $A_{n}$ or of a $B_{i}$ across $B_{n}$.

(4) Stabilizations of the bridge diagram.

As usual, invariance under isotopies is easy: isotopies of the $A_{i}$ and $B_{i}$ give isotopies of the Lagrangians $\widetilde{\mathcal{K}}_{A}$ and $\widetilde{\mathcal{K}}_{B}$.

Handlesliding $A_{n}$ or $B_{n}$ over another curve has no effect at all on the Lagrangians. So, suppose that $A_{i}$ and $A_{i}^{\prime}$ are related by a handleslide over $A_{j}$, where $i, j<n$ : the case of handleslides among $B_{1}, \ldots, B_{n-1}$ is symmetric. Choose $A_{i}^{\prime}$ so that it intersects $A_{i}$ only at its 

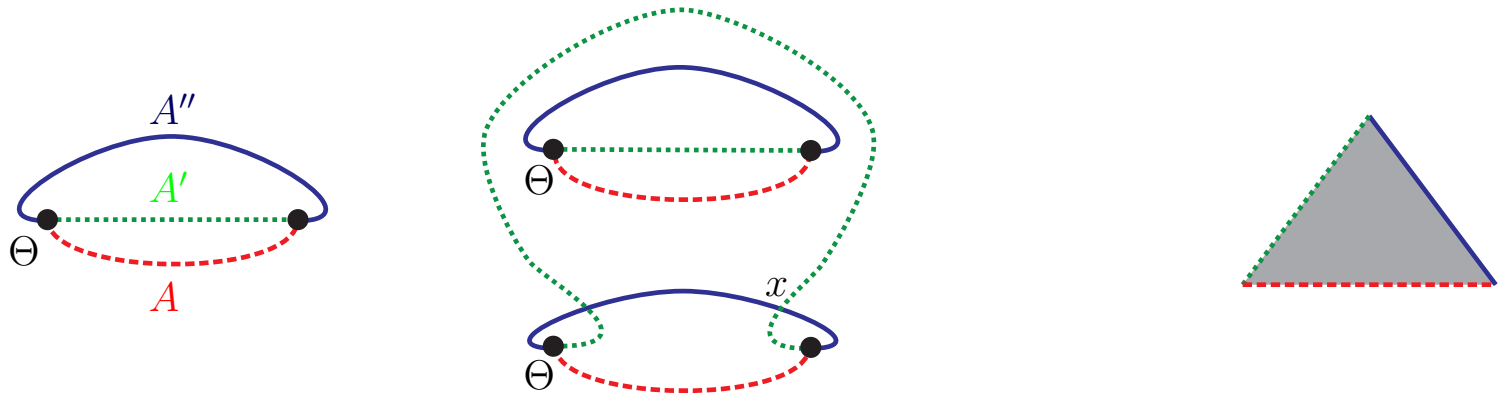

(a)

(b)

Figure 17. A triple diagram for a handleslide. (a) Part of the triple $\left(A, A^{\prime}, A^{\prime \prime}\right)$. $A$ is dashed (red), $A^{\prime}$ is dotted (green), and $A^{\prime \prime}$ is solid (blue). The $\Theta_{\mathrm{s}}$ denote $\Theta_{A, A^{\prime}}$ or $\Theta_{A, A^{\prime \prime}}$. (b) Orientation convention for triangle maps.

endpoints (and transversally) and is disjoint from $A_{k}$ for $k \neq i$. For $k \neq i$ let $A_{k}^{\prime}$ be a small pushoff of $A_{k}$ intersecting $A_{k}$ only at its endpoints (and transversally). Let $\widetilde{\mathcal{K}}_{A}^{\prime}=\Sigma_{A_{1}^{\prime}} \times \cdots \times$ $\Sigma_{A_{i}^{\prime}} \times \cdots \times \Sigma_{A_{n-1}^{\prime}}$. (See the top row of Figure 16.) To prove that $H F\left(\widetilde{\mathcal{K}}_{A}, \widetilde{\mathcal{K}}_{B}\right) \cong H F\left(\widetilde{\mathcal{K}}_{A}^{\prime}, \widetilde{\mathcal{K}}_{B}\right)$ one could verify that the construction of Seidel-Smith's Hamiltonian isotopy [SS06, Lemma 48] restricts to give a Hamiltonian isotopy from $\widetilde{\mathcal{K}}_{A}$ to $\widetilde{\mathcal{K}}_{A}^{\prime}$. Instead, however, we will adapt Ozsváth-Szabó's handleslide invariance proof for Heegaard Floer homology [OSz04b, Section $9]$, which boils down to showing that $\widetilde{\mathcal{K}}_{A}$ and $\widetilde{\mathcal{K}}_{A}^{\prime}$ are isomorphic objects of the Fukaya category.

As a relatively graded abelian group, $C F\left(\widetilde{\mathcal{K}}_{A}, \widetilde{\mathcal{K}}_{A}^{\prime}\right)$ is isomorphic to $H_{*}\left(S^{2}\right)^{\otimes(n-1)}$. In particular, the differential on $C F\left(\widetilde{\mathcal{K}}_{A}, \widetilde{\mathcal{K}}_{A}^{\prime}\right)$ must vanish for grading reasons. For $k \neq i$ let $A_{k}^{\prime \prime}$ be a small deformation of $A_{k}$, intersecting each of $A_{k}$ and $A_{k}^{\prime}$ only in the endpoints, and so that $A_{k}^{\prime}$ is between $A_{k}$ and $A_{k}^{\prime \prime}$. Choose $A_{i}^{\prime \prime}$ similarly, but here $A_{i}^{\prime \prime}$ necessarily intersects $A_{i}^{\prime}$ in two interior points (as well as both endpoints); see Figure 17 . Let $\widetilde{\mathcal{K}}_{A}^{\prime \prime}$ be the corresponding Lagrangian. The top-graded generator $\Theta_{A, A^{\prime \prime}} \in C F\left(\widetilde{\mathcal{K}}_{A}, \widetilde{\mathcal{K}}_{A}^{\prime \prime}\right)$ represents the identity map of $\widetilde{\mathcal{K}}_{A}$ in the Fukaya category. We will show that if $\Theta_{A, A^{\prime}} \in C F\left(\widetilde{\mathcal{K}}_{A}, \widetilde{\mathcal{K}}_{A}^{\prime}\right)$ is the top-graded generator and $\Theta_{A^{\prime}, A^{\prime \prime}} \in C F\left(\widetilde{\mathcal{K}}_{A}^{\prime}, \widetilde{\mathcal{K}}_{A}^{\prime \prime}\right)$ is a chain representing the top-graded homology class then the composition (via triangle maps) $\Theta_{A^{\prime}, A^{\prime \prime}} \circ \Theta_{A, A^{\prime}}=\Theta_{A, A^{\prime \prime}}$. A symmetric argument shows that the composition the other way is the identity element of $\widetilde{\mathcal{K}}_{A}^{\prime}$, implying that $\Theta_{A, A^{\prime}}$ and $\Theta_{A^{\prime}, A^{\prime \prime}}$ are the desired isomorphisms between $\widetilde{\mathcal{K}}_{A}$ and $\widetilde{\mathcal{K}}_{A}^{\prime}$.

To see that $\Theta_{A^{\prime}, A^{\prime \prime}} \circ \Theta_{A, A^{\prime}}=\Theta_{A, A^{\prime \prime}}$, arrange $A, A^{\prime}$, and $A^{\prime \prime}$ as in Figure 17. In particular, $\Theta_{A, A^{\prime}}$ and $\Theta_{A, A^{\prime \prime}}$ are both the tuple of left endpoints of the $n A_{k}$-arcs. The generator $\Theta_{A^{\prime}, A^{\prime \prime}}$ is represented by a cycle which is the sum of this tuple and some other tuples, each of which has at least one point projecting to the intersection marked $x$ in the figure. (See the proof of Lemma 7.17 for a discussion of computing the gradings of generators.)

The constant triangles at $\Theta_{A, A^{\prime}}=\Theta_{A, A^{\prime \prime}}$ are, in fact, transversely cut out and give one contribution of $\Theta_{A, A^{\prime \prime}}$ in $\Theta_{A^{\prime}, A^{\prime \prime}} \circ \Theta_{A, A^{\prime}}$. (One way to see this is to note that the local picture near $\Theta_{A, A^{\prime}}$ is the same as if $A^{\prime}$ were a small Hamiltonian-isotopic copy of $A$, and the constant triangles contribute in the case of a small Hamiltonian isotopy. Alternately, lift $A, A^{\prime}$, and $A^{\prime \prime}$ to the branched double cover, perturb away the triple point, and note that 

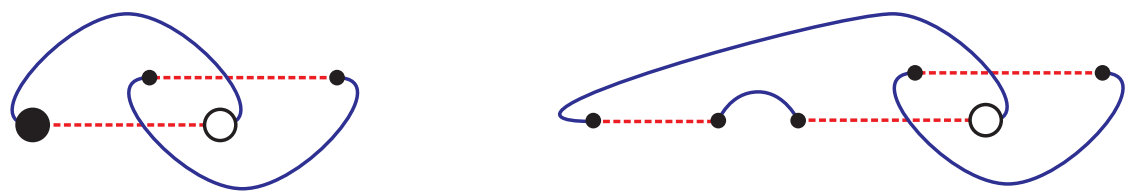

FIGURE 18. Stabilizing when the basepoint is on a one-bridge unknot. The case of the Hopf link is shown, but the un-marked components could, of course, be more complicated. The key features are that the marked component is a one-bridge unknot and the point $p_{2 n-1}$, which is drawn as a large black dot, is adjacent to the non-compact region in $\mathbb{C}$. The basepoint $p_{2 n}$ is drawn as a large white dot in both pictures.

there is a unique small triangle connecting the three generators.) It remains to see that no other triangles cancel these. However, we can read off the (projections to $\mathbb{C}$ of) corners of holomorphic triangles from the projected domain, and there is no other projected domain with non-negative multiplicities and corners at $\Theta_{A, A^{\prime}}, \Theta_{A, A^{\prime \prime}}$, and points in $\Theta_{A^{\prime}, A^{\prime \prime}}$. Thus, $\Theta_{A^{\prime}, A^{\prime \prime}} \circ \Theta_{A, A^{\prime}}=\Theta_{A, A^{\prime \prime}}$, as desired.

The same argument works for handlesliding $A_{i}$ over $A_{n}$.

Turning to stabilization invariance, recall that stabilizations of the arc diagram occur at one of the $p_{i}$. Since we have already verified isotopy and handleslide invariance, it suffices to prove stabilization invariance at one $p_{i}$ for each link component. So, if the link component containing $p_{2 n}$ involves at least $2 A_{i}$-arcs then it suffices to prove stabilization at a $p_{i}$ which is not an endpoint of $A_{n}$ or $B_{n}$. In this case, we can perform a sequence of handleslides and isotopies so that the $p_{i}$ is adjacent to the non-compact region in $\mathbb{R}^{2}$, and then the neck-stretching argument from Section 7.4 implies stabilization invariance.

The remaining case is that $A_{n}$ and $B_{n}$ together form an unknot component. Let $p_{2 n-1}$ and $p_{2 n}$ denote the endpoints of $A_{n}$ (and $B_{n}$ ); $p_{2 n}$ is the basepoint, and we will stabilize at $p_{2 n-1}$. In this case, perform a sequence of handleslides and isotopies so that $p_{2 n-1}$ is adjacent to the non-compact region and $A_{n}$ and $B_{n}$ only intersect at their endpoints (as in Figure 18). In the stabilized diagram, the unknot component has two $A_{i}$-arcs and two $B_{i}$-arcs. Of these four arcs, the two that do not contain the basepoint $p_{2 n}$ are adjacent to the non-compact region. Therefore it easily follows that the chain complex after the stabilization is chain isomorphic to the chain complex before the stabilization. See Figure 18.

Remark 7.26. Abouzaid-Smith's techniques [AS16,AS15] presumably also lead to a proof of Theorem 7.25.

7.6. Some conjectures. We start by recalling some earlier conjectures. First, Seidel-Smith conjectured a relationship between symplectic Khovanov homology and combinatorial Khovanov homology:

Conjecture 7.27. [SSO6, Conjecture 2] $K h^{\text {symp }, \ell}(L) \cong \oplus_{i-j=\ell} K h^{i, j}(L)$.

(The Khovanov differential has bigrading $(1,0)$.) Abouzaid-Smith recently proved this conjecture in characteristic 0 [AS15]; at the time of writing, the positive characteristic case remains open.

Perhaps inspired by Lee's deformation of the Khovanov complex [Lee05], Bar-Natan constructed [BN05] a bigraded chain complex $C_{B N}(L)$ freely generated over $\mathbb{F}_{2}[H]$, with 
the differential in bigrading $(1,0)$ and the formal variable $H$ in bigrading $(0,-2)$, satisfying the following: the chain homotopy type of $C_{B N}(L)$ over $\mathbb{F}_{2}[H]$ is a link invariant; $C_{B N}(L) /\{H=0\}=C_{K h}(L)$; and the homology of the localized complex $H^{-1} C_{B N}(L)$ is $2^{|L|}$ copies of $\mathbb{F}_{2}\left[H, H^{-1}\right]$. On the symplectic side, if we consider the action of $\mathbb{Z} / 2 \subset S O(2)$ on $\mathcal{Y}_{n}$ then by Theorem $7.16, \alpha^{-1} H F_{\mathbb{Z} / 2}\left(\mathcal{K}_{A}^{\prime}, \mathcal{K}_{B}\right)$ is isomorphic to $2^{|L|}$ copies of $\mathbb{F}_{2}\left[\alpha, \alpha^{-1}\right]$. Therefore, the following seems to be a reasonable conjecture.

Conjecture 7.28. There is an ungraded isomorphism of $\mathbb{F}_{2}[H] \cong \mathbb{F}_{2}[\alpha]$-modules

$$
H F_{\mathbb{Z} / 2}\left(\mathcal{K}_{A}^{\prime}, \mathcal{K}_{B}\right) \cong H_{*}\left(C_{B N}(L)\right) \text {. }
$$

Question 7.29. Is there a refinement of the above conjecture relating the single grading of $H F_{\mathbb{Z} / 2}\left(\mathcal{K}_{A}^{\prime}, \mathcal{K}_{B}\right)$ and the bigrading of $C_{B N}(L)$ ?

On a parallel front, Szabó constructed a bigraded chain complex $C_{S z}(L)$ freely generated over $\mathbb{F}_{2}[W]$, with the differential in bigrading $(1,0)$ and the formal variable $W$ in bigrading $(-1,-2)$, so that $C_{K h}(L)=C_{S z}(L) /(W=0)$, and the chain homotopy type of $C_{S z}(L)$ over $\mathbb{F}_{2}[W]$ is a link invariant [Sza15]. Moreover, he conjectured that the chain complex $C_{S z}(L)$ should be chain homotopy equivalent to a similar looking chain complex constructed earlier by Ozsváth-Szabó [OSz05] via holomorphic curves. On the symplectic side, SeidelSmith used the $\mathbb{Z} / 2=\{1, \tau\}$ action coming from $\tau(u, v)=(u,-v)$ (Equation (7.4)) to construct the $\mathbb{Z} / 2$-equivariant symplectic Khovanov cohomology $H F_{\{1, \tau\}}\left(\mathcal{K}_{A}^{\prime}, \mathcal{K}_{B}\right)$ as a module over $H^{*}(\mathbb{Z} / 2)\left(=\mathbb{F}_{2}[W]\right.$ say $)$, and propose [SS10, Section 4.5] that the resulting spectral sequence from $K h_{\text {symp }}(L)$ may be isomorphic to the Ozsváth-Szabó spectral sequence $K h(L) \Rightarrow \widehat{H F}\left(\Sigma(m(L)) \#\left(S^{2} \times S^{1}\right)\right)$ [OSz05]. Combining the two conjectures, we get:

Conjecture 7.30 (Seidel-Smith-Szabó). As modules over $\mathbb{F}_{2}[W], H F_{\{1, \tau\}}\left(\mathcal{K}_{A}^{\prime}, \mathcal{K}_{B}\right)$ is isomorphic to the homology of $C_{S z}(L)$ after collapsing the bigrading $(i, j)$ into a single grading $i-j$.

Finally, Sarkar-Seed-Szabó combined $C_{B N}(L)$ and $C_{S z}(L)$ into a single bigraded chain complex $C_{\text {tot }}(L)$, freely generated over a polynomial ring $\mathbb{F}_{2}[H, W]$, with the differential in bigrading $(1,0)$, and the formal variables $H$ and $W$ in bigradings $(0,-2)$ and $(-1,-2)$, respectively [SSS14]. The construction satisfies the following properties (listed in [SSS14, Section 1]):

- The chain homotopy type of $C_{t o t}(L)$ over $\mathbb{F}_{2}[H, W]$ is a link invariant.

- $C_{B N}(L)=C_{t o t}(L) /(W=0)$ and $C_{S z}(L)=C_{t o t}(L) /(H=0)$; in particular, $C_{K h}(L)=$ $C_{\text {tot }}(L) /(H=W=0)$.

- The homology of $H^{-1} \mathcal{C}_{t o t}$ is $2^{|L|}$ copies of $\mathbb{F}_{2}\left[H, H^{-1}, W\right]$.

On the symplectic side, there is an $O(2)$-action, and we may restrict to dihedral group

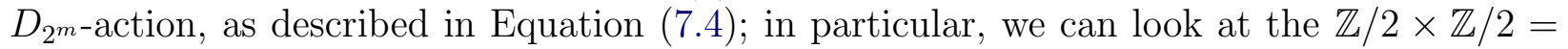
$\{1, \sigma, \tau, \sigma \tau\}$ action combining the above two actions.

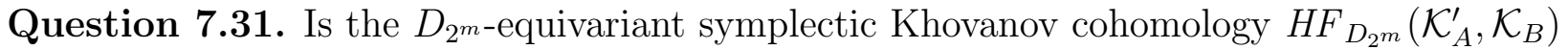
(in particular, the $\mathbb{Z} / 2 \times \mathbb{Z} / 2$-equivariant Floer cohomology) related to $C_{\text {tot }}(L)$ ? If so, how are the module structures (over $H^{*}\left(D_{2^{m}}\right)$ and $\mathbb{F}_{2}[H, W]$, respectively) related? How do the single grading on the symplectic side and the bigrading on the combinatorial side interact? 


\section{Appendix A. Instructive Examples}

The examples in this section are intended to help the reader unfamiliar with homotopy coherence to come to terms with the notions used in this paper. Vogt's original paper [Vog73] and the Kamps-Porter's book [KP97] are also pleasantly written, and the reader is encouraged to consult them for more information.

We start with examples illustrating homotopy coherent diagrams for almost complex structures and complexes, Definitions 3.3 and 3.7, respectively.

Example A.1. The first few terms of a $\mathscr{E} \mathbb{Z} / 2$-diagram in $\overline{\mathcal{J}}$ consist of:

(1) Two cylindrical almost complex structures $F(a)=J_{a}$ and $F(b)=J_{b}$.

(2) Two (equivalence classes of sequences of) eventually cylindrical complex structures $F(\alpha)=\widetilde{J}_{\alpha} \in \overline{\mathcal{J}}\left(J_{a}, J_{b}\right)$, and $F(\beta)=\widetilde{J}_{\beta} \in \overline{\mathcal{J}}\left(J_{b}, J_{a}\right)$, and two others associated to the identity maps and equal to $J_{a}$ and $J_{b}$.

(3) Two smooth families of eventually cylindrical complex structures indexed by $t \in[0,1]$ :

$$
F(\alpha, \beta)=\widetilde{J}_{(\alpha, \beta, t)} \in \overline{\mathcal{J}}\left(J_{b}, J_{b}\right) \quad F(\beta, \alpha)=\widetilde{J}_{(\beta, \alpha, t)} \in \overline{\mathcal{J}}\left(J_{a}, J_{a}\right)
$$

which satisfy:

$$
\begin{aligned}
\widetilde{J}_{(\alpha, \beta, 1)} & =\widetilde{J}_{\alpha \circ \beta}=\widetilde{J}_{\mathbb{I}_{b}}=J_{b} & \widetilde{J}_{(\alpha, \beta, 0)} & =\widetilde{J}_{\alpha} \circ \widetilde{J}_{\beta}=\left(\widetilde{J}_{\beta}, \widetilde{J}_{\alpha}\right) \\
\widetilde{J}_{(\beta, \alpha, 1)} & =\widetilde{J}_{\beta \circ \alpha}=\widetilde{J}_{\mathbb{I}_{a}}=J_{a} & \widetilde{J}_{(\beta, \alpha, 0)} & =\widetilde{J}_{\beta} \circ \widetilde{J}_{\alpha}=\left(\widetilde{J}_{\alpha}, \widetilde{J}_{\beta}\right) .
\end{aligned}
$$

and six other $[0,1]$-families of almost complex structures determined by the data of the first two steps.

(4) Two smooth families of cylindrical-at-infinity complex structures indexed by $\left(t_{1}, t_{2}\right) \in$ $[0,1]^{2}$ :

$$
\begin{array}{rlrl}
F(\alpha, \beta, \alpha) & =\widetilde{J}_{\left(\alpha, \beta, \alpha, t_{1}, t_{2}\right)} \in \overline{\mathcal{J}}\left(J_{a}, J_{a}\right) & F(\beta, \alpha, \beta) & =\widetilde{J}_{\left(\beta, \alpha, \beta, t_{1}, t_{2}\right)} \in \overline{\mathcal{J}}\left(J_{b}, J_{b}\right) \\
\text { which satisfy: } & & \widetilde{J}_{\left(\alpha, \beta, \alpha, t_{1}, 1\right)} & =\widetilde{J}_{\left(\alpha \circ \beta, \alpha, t_{1}\right)}=\widetilde{J}_{\left(\mathbb{I}_{b}, \alpha, t_{1}\right)}=\widetilde{J}_{\alpha} \\
\widetilde{J}_{\left(\alpha, \beta, \alpha, 1, t_{2}\right)} & =\widetilde{J}_{\left(\alpha, \beta \circ \alpha, t_{2}\right)}=\widetilde{J}_{\left(\alpha, \mathbb{I}_{a}, t_{2}\right)}=\widetilde{J}_{\alpha} & \widetilde{J}_{\left(\alpha, \beta, \alpha, t_{1}, 0\right)} & =\left(\widetilde{J}_{\left(\beta, \alpha, t_{2}\right)}, \widetilde{J}_{\alpha}\right) \\
\widetilde{J}_{\left(\alpha, \beta, \alpha, 0, t_{2}\right)} & =\left(\widetilde{J}_{\alpha}, \widetilde{J}_{\left(\alpha, \beta, t_{2}\right)}\right) & \widetilde{J}_{\left(\beta, \alpha, \beta, t_{1}, 1\right)} & =\widetilde{J}_{\left(\beta \circ \alpha, \beta, t_{1}\right)}=\widetilde{J}_{\left(\mathbb{I}_{a}, \beta, t_{1}\right)}=\widetilde{J}_{\beta} \\
\widetilde{J}_{\left(\beta, \alpha, \beta, 1, t_{2}\right)} & =\widetilde{J}_{\left(\beta, \alpha \circ \beta, t_{2}\right)}=\widetilde{J}_{\left(\beta, \mathbb{I}_{b}, t_{2}\right)}=\widetilde{J}_{\beta} & \widetilde{J}_{\left(\beta, \alpha, \beta, t_{1}, 0\right)} & =\left(\widetilde{J}_{\left(\alpha, \beta, t_{2}\right)}, \widetilde{J}_{\beta}\right) \\
\widetilde{J}_{\left(\beta, \alpha, \beta, 0, t_{2}\right)} & =\left(\widetilde{J}_{\beta}, \widetilde{J}_{\left(\beta, \alpha, t_{2}\right)}\right) &
\end{array}
$$

and fourteen other $[0,1]^{2}$-families of almost complex structures determined by the data of the first three steps.

Example A.2. The first few terms of a homotopy coherent $\mathscr{E} \mathbb{Z} / 2$-diagram in Kom consist of:

(1) Two chain complexes $G(a)$ and $G(b)$ over $\mathbb{F}_{2}$.

(2) Two chain maps $G_{\alpha}: G(a) \rightarrow G(b)$ and $G_{\beta}: G(b) \rightarrow G(a)$.

(3) Two maps

$$
G_{\beta, \alpha}: G(a) \rightarrow G(a) \quad G_{\alpha, \beta}: G(b) \rightarrow G(b)
$$

which satisfy:

$$
\partial \circ G_{\beta, \alpha}=G_{\beta, \alpha} \circ \partial+G_{\beta} \circ G_{\alpha}+\mathbb{I}_{G(a)} \quad \partial \circ G_{\alpha, \beta}=G_{\alpha, \beta} \circ \partial+G_{\alpha} \circ G_{\beta}+\mathbb{I}_{G(b)} .
$$


(4) Two maps

$$
G_{\alpha, \beta, \alpha}: G(a) \rightarrow G(a) \quad G_{\beta, \alpha, \beta}: G(b) \rightarrow G(b)
$$

which satisfy:

$$
\begin{aligned}
& \partial \circ G_{\alpha, \beta, \alpha}=G_{\alpha, \beta, \alpha} \circ \partial+G_{\alpha, \beta} \circ G_{\alpha}+G_{\alpha} \circ G_{\beta, \alpha} \\
& \partial \circ G_{\beta, \alpha, \beta}=G_{\beta, \alpha, \beta} \circ \partial+G_{\beta, \alpha} \circ G_{\beta}+G_{\beta} \circ G_{\alpha, \beta} .
\end{aligned}
$$

The following example illustrates homotopy colimits, Definition 3.11. In particular, we observe that a notion of iterated mapping cones defined using homotopy colimits agrees with a more direct definition in terms of complexes.

Example A.3. Let $\mathscr{C}$ be the category

$$
\mathscr{C}=\left.f_{2}\right|_{\longleftarrow} ^{\leftarrow} f_{f_{4}}^{f_{1}} \bullet
$$

A homotopy coherent $\mathscr{C}$-diagram $G: \mathscr{C} \rightarrow$ Kom induces a diagram

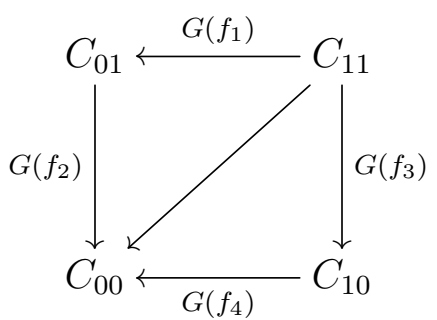

where the diagonal map is $G_{f_{2}, f_{1}}+G_{f_{4}, f_{3}}$. Since $G_{f_{2}, f_{1}}$ is a homotopy between $G\left(f_{2}\right) \circ G\left(f_{1}\right)$ and $G\left(f_{2} \circ f_{1}\right)$ and $G_{f_{4}, f_{3}}$ is a homotopy between $G\left(f_{4} \circ f_{3}\right)$ and $G\left(f_{4}\right) \circ G\left(f_{3}\right)$, the diagonal map is a homotopy between $G\left(f_{2}\right) \circ G\left(f_{1}\right)$ and $G\left(f_{4}\right) \circ G\left(f_{3}\right)$.

Let $\mathscr{C}_{+}$be the result of adding a single object $*$ to $\mathscr{C}$ and a morphism from each nonterminal vertex of $\mathscr{C}$ to 0 . The homotopy coherent diagram $G$ extends uniquely to a homotopy coherent diagram $G_{+}: \mathscr{C}_{+} \rightarrow$ Kom with $G_{+}(*)=0$ the zero complex. We label the arrows as:

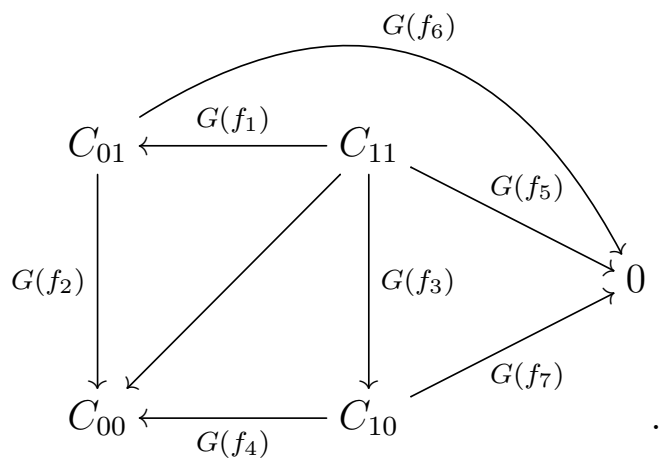

We will show that the homotopy colimit of $G_{+}$is quasi-isomorphic to the total mapping cone of the diagram (A.4). 
First, let us consider the general form of some basic differentials in the homotopy colimit. Let $f: a \rightarrow b$ be a morphism in the original category $\mathscr{C}$. Let $x \in G(a)$. Then

$$
\begin{aligned}
\partial(G(f) ;\{0,1\} ; x) & =(G(f) ;\{0,1\} ; \partial x)+(G(f) ;\{1\} ; x)+(G(f) ;\{0\} ; x) \\
& =(G(f) ;\{0,1\} ; \partial x)+x+G(f)(x) .
\end{aligned}
$$

Note that if $G(b)=0$ then $G(f)(x)=0$ automatically, so we have $d(G(f) ;\{0,1\} ; x)=$ $(G(f) ;\{0,1\} ; \partial x)+x$. Schematically, we draw the following picture:

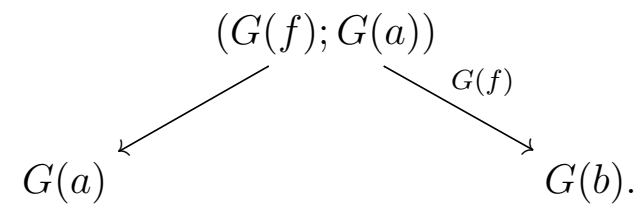

Next, let $g: b \rightarrow c$ be another morphism composable with $f$. Then we have

$$
\begin{aligned}
\partial(G(g, f) ;\{0,1\} \otimes\{0,1\} ; x)=(G(g, f) ; & \{0,1\} \otimes\{0,1\} ; \partial x)+(G(f) ;\{0,1\} ; x) \\
& +(G(g \circ f) ;\{0,1\} ; x)+(G(g) ;\{0,1\} ; G(f)(x)) \\
& +G_{g, f}(x) .
\end{aligned}
$$

Notice that if $G(b)=0$, then $G(g, f)(x)=0$ and the last term vanishes. (However, importantly, $(G(g \circ f) ;\{0,1\} ; x)$ may be nonzero, even though $g \circ f$ is a zero map.) Schematically we draw the following picture:

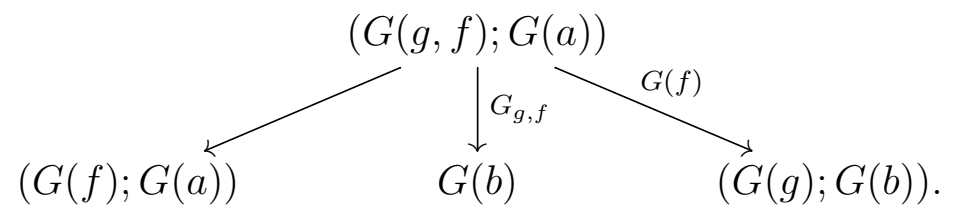

Now let us look at the homotopy colimit of the homotopy-coherent diagram (A.5). As a vector space, it is the direct sum of the following sixteen terms.

- The four chain complexes $C_{11}, C_{01}, C_{10}$, and $C_{00}$.

- Terms corresponding to the eight morphisms in the diagram, to wit,

$$
\begin{array}{llll}
\left(G\left(f_{1}\right) ;\{0,1\} ; C_{11}\right) & \left(G\left(f_{2}\right) ;\{0,1\} ; C_{01}\right) & \left(G\left(f_{3}\right) ;\{0,1\} ; C_{11}\right) & \left(G\left(f_{4}\right) ;\{0,1\} ; C_{10}\right) \\
\left(G\left(f_{5}\right) ;\{0,1\} ; C_{11}\right) & \left(G\left(f_{6}\right) ;\{0,1\} ; C_{01}\right) & \left(G\left(f_{7}\right) ;\{0,1\} ; C_{10}\right) & \left(G\left(f_{2}, f_{1}\right) ;\{0,1\} ; C_{11}\right) .
\end{array}
$$

- Four terms corresponding to pairs of composable morphisms, to wit,

$$
\begin{array}{ll}
\left(G\left(f_{2} ; f_{1}\right) ;\{0,1\} \otimes\{0,1\} ; C_{11}\right) & \left(G\left(f_{4} ; f_{3}\right) ;\{0,1\} \otimes\{0,1\} ; C_{11}\right) \\
\left(G\left(f_{6} ; f_{1}\right) ;\{0,1\} \otimes\{0,1\} ; C_{11}\right) & \left(G\left(f_{7} ; f_{3}\right) ;\{0,1\} \otimes\{0,1\} ; C_{11}\right) .
\end{array}
$$

From here on, no terms in $I_{*}^{\otimes n}$ will appear other than $\{0,1\}$ and $\{0,1\} \otimes\{0,1\}$, and which is appropriate will be clear from context. So, in the interests of readability we will omit 
these terms. The differentials on the homotopy colimit are as follows:

$$
\begin{aligned}
& \partial\left(G\left(f_{2}, f_{1}\right) ; x\right)=\left(G\left(f_{2}, f_{1}\right) ; \partial x\right)+\left(G\left(f_{1}\right) ; x\right)+\left(G\left(f_{2} \circ f_{1}\right) ; x\right)+\left(G\left(f_{2}\right) ; G\left(f_{1}\right) x\right)+G_{f_{2}, f_{1}} x \\
& \partial\left(G\left(f_{4}, f_{3}\right) ; x\right)=\left(G\left(f_{4}, f_{3}\right) ; \partial x\right)+\left(G\left(f_{3}\right) ; x\right)+\left(G\left(f_{2} \circ f_{1}\right) ; x\right)+\left(G\left(f_{4}\right) ; G\left(f_{3}\right) x\right)+G_{f_{4}, f_{3}} x \\
& \partial\left(G\left(f_{6}, f_{1}\right) ;\right)=\left(G\left(f_{6}, f_{1}\right) ; \partial x\right)+\left(G\left(f_{5}\right) ; x\right)+\left(G\left(f_{1}\right) ; x\right)+\left(G\left(f_{6}\right) ; G\left(f_{1}\right) x\right) \\
& \partial\left(G\left(f_{7}, f_{3}\right) ; x\right)=\left(G\left(f_{7}, f_{3} ; \partial x\right)+\left(G\left(f_{5}\right) ; x\right)+\left(G\left(f_{3}\right) ; x\right)+\left(G\left(f_{6}\right) ; G\left(f_{3}\right) x\right)\right. \\
& \partial\left(G\left(f_{2} \circ f_{1}\right) ; x\right)=\left(G\left(f_{2} \circ f_{1}\right) ; \partial x\right)+x+G\left(f_{2} \circ f_{1}\right) x \\
& \partial\left(G\left(f_{1}\right) ; x\right)=\left(G\left(f_{1}\right) ; \partial x\right)+x+G\left(f_{1}\right) x \\
& \partial\left(G\left(f_{2}\right) ; x\right)=\left(G\left(f_{2}\right) ; \partial x\right)+x+G\left(f_{2}\right) x \\
& \partial\left(G\left(f_{3}\right) ; x\right)=\left(G\left(f_{3}\right) ; \partial x\right)+x+G\left(f_{3}\right) x \\
& \partial\left(G\left(f_{4}\right) ; x\right)=\left(G\left(f_{4}\right) ; \partial x\right)+x+G\left(f_{4}\right) x \\
& \partial\left(G\left(f_{5}\right) ; x\right)=\left(G\left(f_{5}\right) ; \partial x\right)+x \\
& \partial\left(G\left(f_{6}\right) ; x\right)=\left(G\left(f_{6}\right) ; \partial x\right)+x \\
& \partial\left(G\left(f_{7} ; x\right)=\left(G\left(f_{7}\right) ; \partial x\right)+x\right.
\end{aligned}
$$

We will break the total complex into seven subcomplexes, six of which are acyclic and one of which is the mapping cone. The six trivial subcomplexes are split off by edge reductions along blank arrows in the homotopy colimit expressed using the diagrammatic conventions explained above; the interested reader is encouraged to draw the diagram and follow along.

Our first subcomplex $C_{1}$ has a basis consisting of all elements

$$
\begin{aligned}
& a_{x}^{1}=\left(G\left(f_{6}, f_{1}\right) ; x\right) \\
& \left.b_{x}^{1}=\left(G\left(f_{5}\right) ; x\right)+G\left(f_{1}\right) ; x\right)+\left(G\left(f_{6}\right) ; G\left(f_{1}\right) x\right)+\left(G\left(f_{6}, f_{1}\right) ; \partial x\right)
\end{aligned}
$$

for $x$ an element of $C_{11}$. Then the differential $\partial$ on this complex is $\partial\left(a_{x}^{1}\right)=b_{x}^{1}$ and $\partial\left(b_{x}^{1}\right)=0$.

Our second subcomplex $C_{2}$ has a basis consisting of all elements

$$
\begin{aligned}
a_{x}^{2}= & \left(G\left(f_{7}, f_{3}\right) ; x\right)+\left(G\left(f_{6}, f_{1}\right) ; x\right) \\
b_{x}^{2}= & \left(G\left(f_{1}\right) ; x\right)+\left(G\left(f_{3}\right) ; x\right)+\left(G\left(f_{6}\right) ; G\left(f_{1}\right) x\right) \\
& +\left(G\left(f_{7}\right) ; G\left(f_{3}\right) x\right)+\left(G\left(f_{7}, f_{3}\right) ; \partial x\right)+\left(G\left(f_{6}, f_{1}\right) ; \partial x\right)
\end{aligned}
$$

for $x \in C_{11}$. As previously, $\partial\left(a_{x}^{2}\right)=b_{x}^{2}$ and $\partial\left(b_{x}^{2}\right)=0$.

Our third subcomplex $C_{3}$ has basis consisting of all elements

$$
\begin{aligned}
a_{x}^{3}= & \left(G\left(f_{2}, f_{1}\right) ; x\right)+\left(G\left(f_{7}, f_{3}\right) ; x\right)+\left(G\left(f_{6}, f_{1}\right) ; x\right) \\
b_{x}^{3}= & \left(G\left(f_{3}\right) ; x\right)+\left(G\left(f_{2} \circ f_{1}\right) ; x\right)+\left(G\left(f_{6}\right) ; G\left(f_{1}\right) x\right) \\
& +\left(G\left(f_{2}\right) ; G\left(f_{1}\right) x\right)+\left(G\left(f_{7}\right) ; G\left(f_{3}\right) x\right)+G_{f_{2}, f_{1}} x \\
& +\left(G\left(f_{2}, f_{1}\right) ; \partial x\right)+\left(G\left(f_{7}, f_{3}\right) ; \partial x\right)+\left(G\left(f_{6}, f_{1}\right) ; \partial x\right)
\end{aligned}
$$

for $x \in C_{11}$. As previously, $\partial\left(a_{x}^{3}\right)=b_{x}^{3}$ and $\partial\left(b_{x}^{3}\right)=0$.

Our fourth subcomplex $C_{4}$ has a basis consisting of all elements

$$
\begin{aligned}
& a_{x}^{4}=\left(G\left(f_{2} \circ f_{1}\right) ; x\right) \\
& b_{x}^{4}=x+G\left(f_{2} \circ f_{1}\right) x+\left(G\left(f_{2} \circ f_{1}\right) ; \partial x\right)
\end{aligned}
$$

for $x \in C_{11}$. As previously, $\partial\left(a_{x}^{4}\right)=b_{x}^{4}$ and $\partial\left(b_{x}^{4}\right)=0$. 
Our fifth subcomplex $C_{5}$ has a basis consisting of all elements

$$
\begin{aligned}
& a_{x}^{5}=\left(G\left(f_{6}\right) ; x\right) \\
& b_{x}^{5}=x+\left(G\left(f_{6}\right) ; \partial x\right)
\end{aligned}
$$

for $x \in C_{01}$. As previously, $\partial\left(a_{x}^{5}\right)=b_{x}^{5}$ and $\partial\left(b_{x}^{5}\right)=0$.

Our sixth subcomplex $C_{6}$ has a basis consisting of all elements

$$
\begin{aligned}
& a_{x}^{6}=\left(G\left(f_{7}\right) ; x\right) \\
& b_{x}^{6}=x+\left(G\left(f_{7}\right) ; \partial x\right)
\end{aligned}
$$

for $x \in C_{10}$. As previously, $\partial\left(a_{x}^{6}\right)=b_{x}^{6}$ and $\partial\left(b_{x}^{6}\right)=0$.

Finally we come to the subcomplex remaining after splitting all six of these acyclic complexes. $C_{7}$ has a basis consisting of elements

$$
\begin{aligned}
a_{x}^{7} & =\left(G\left(f_{4}, f_{3}\right) ; x\right)+\left(G\left(f_{2}, f_{1}\right) ; x\right)+\left(G\left(f_{7}, f_{3}\right) ; x\right)+\left(G\left(f_{6}, f_{1}\right) ; x\right), \quad x \in C_{11} \\
b_{x}^{7} & =\left(G\left(f_{6}\right) ; x\right)+\left(G\left(f_{2}\right) ; x\right), \quad x \in C_{01} \\
c_{x}^{7} & =\left(G\left(f_{4}\right) ; x\right)+\left(G\left(f_{7}\right) ; x\right), \quad x \in C_{10} \\
e_{x}^{7} & =x, \quad x \in C_{00} .
\end{aligned}
$$

with differentials

$$
\begin{aligned}
& \partial a_{x}^{7}=a_{\partial x}^{7}+b_{G\left(f_{1}\right) x}^{7}+c_{G\left(f_{3}\right) x}^{7}+e_{\left(G_{f_{2}, f_{1}}+G_{f_{4}, f_{3}}\right) x}^{7} \\
& \partial b_{x}^{7}=b_{\partial x}^{7}+e_{G\left(f_{2}\right) x}^{7} \\
& c_{x}^{7}=c_{\partial x}^{7}+d_{G\left(f_{4}\right) x}^{7} \\
& e_{x}^{7}=e_{\partial x}^{7}
\end{aligned}
$$

This is the mapping cone on (A.4). Using the previous diagrammatic conventions, it is the complex shown here.

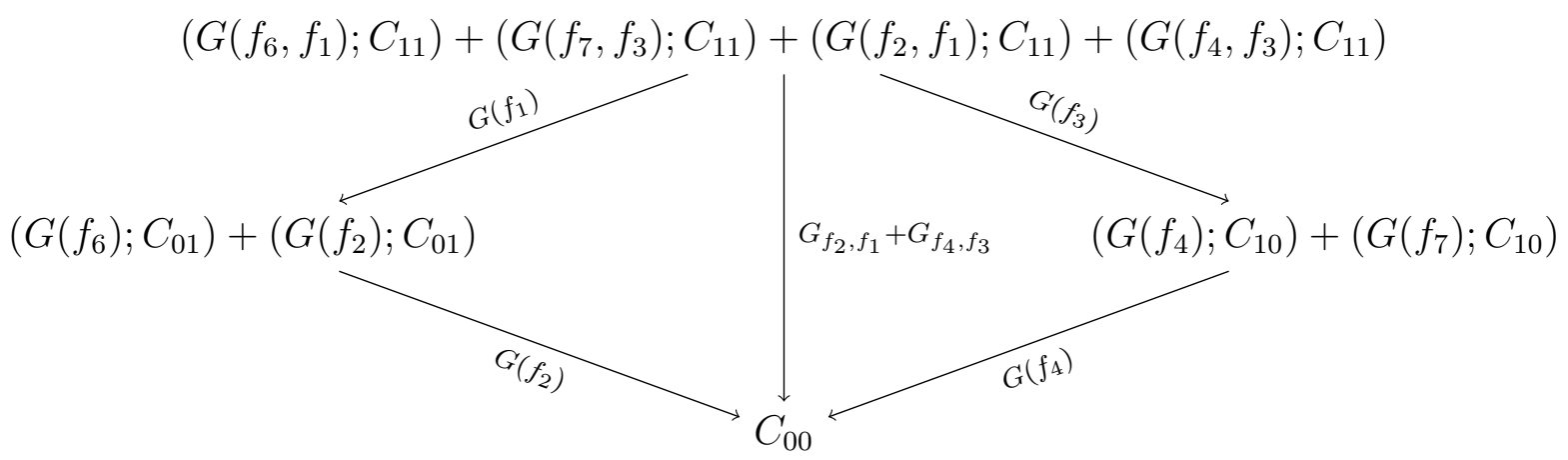

\section{REFERENCES}

[AM04] Alejandro Adem and R. James Milgram, Cohomology of finite groups, second ed., Grundlehren der Mathematischen Wissenschaften [Fundamental Principles of Mathematical Sciences], vol. 309, Springer-Verlag, Berlin, 2004.

[AS15] Mohammed Abouzaid and Ivan Smith, Khovanov homology from Floer cohomology, 2015, arXiv: 1504.01230.

[AS16] Mohammed Abouzaid and Ivan Smith, The symplectic arc algebra is formal, Duke Math. J. 165 (2016), no. 6, 985-1060. 
$\left[\mathrm{BEH}^{+} 03\right]$ Frédéric Bourgeois, Yakov Eliashberg, Helmut Hofer, Kris Wysocki, and Eduard Zehnder, Compactness results in symplectic field theory, Geom. Topol. 7 (2003), 799-888, arXiv:math.SG/ 0308183.

[Bel10] Anna Beliakova, A simplification of combinatorial link Floer homology, J. Knot Theory Ramifications 19 (2010), no. 2, 125-144.

[BK72] A. K. Bousfield and D. M. Kan, Homotopy limits, completions and localizations, Lecture Notes in Mathematics, Vol. 304, Springer-Verlag, Berlin-New York, 1972.

[BN05] Dror Bar-Natan, Khovanov's homology for tangles and cobordisms, Geom. Topol. 9 (2005), 14431499.

[CH13] Cheol-Hyun Cho and Hansol Hong, Finite group actions on Lagrangian Floer theory, 2013, arXiv: 1307.4573.

[CP86] Jean-Marc Cordier and Timothy Porter, Vogt's theorem on categories of homotopy coherent diagrams, Math. Proc. Cambridge Philos. Soc. 100 (1986), no. 1, 65-90.

[EG91] Yakov Eliashberg and Mikhael Gromov, Convex symplectic manifolds, Several complex variables and complex geometry, Part 2 (Santa Cruz, CA, 1989), Proc. Sympos. Pure Math., vol. 52, Amer. Math. Soc., Providence, RI, 1991, pp. 135-162.

[FP78] Zbigniew Fiedorowicz and Stewart Priddy, Homology of classical groups over finite fields and their associated infinite loop spaces, Lecture Notes in Mathematics, vol. 674, Springer, Berlin, 1978.

[Gri06] J. Elisenda Grigsby, Knot Floer homology in cyclic branched covers, Algebr. Geom. Topol. 6 (2006), 1355-1398.

[Hat02] Allen Hatcher, Algebraic topology, Cambridge University Press, Cambridge, 2002.

[Hen12] Kristen Hendricks, A rank inequality for the knot Floer homology of double branched covers, Algebr. Geom. Topol. 12 (2012), no. 4, 2127-2178.

[Hen15] Localization and the link Floer homology of doubly-periodic knots, J. Symplectic Geom. 13 (2015), no. 3, 545-608.

[Hen16] - A spectral sequence of the Floer cohomology of symplectomorphisms of trivial polarization class, Int. Math. Res. Not. IMRN (2016), Art. ID rnw061.

[HLS97] Helmut Hofer, Véronique Lizan, and Jean-Claude Sikorav, On genericity for holomorphic curves in four-dimensional almost-complex manifolds, J. Geom. Anal. 7 (1997), no. 1, 149-159.

[HM15] Kristen Hendricks and Ciprian Manolescu, Involutive Heegaard Floer homology, 2015, arXiv: 1507.00383.

[JT12] András Juhász and Dylan P. Thurston, Naturality and mapping class groups in Heegaard Floer homology, 2012, arXiv:1210.4996.

[Kho00] Mikhail Khovanov, A categorification of the Jones polynomial, Duke Math. J. 101 (2000), no. 3, 359-426.

[KP97] K. H. Kamps and T. Porter, Abstract homotopy and simple homotopy theory, World Scientific Publishing Co., Inc., River Edge, NJ, 1997.

[KS02] Mikhail Khovanov and Paul Seidel, Quivers, Floer cohomology, and braid group actions, J. Amer. Math. Soc. 15 (2002), no. 1, 203-271, arxiv:math.QA/0006056.

[Lee05] Eun Soo Lee, An endomorphism of the Khovanov invariant, Adv. Math. 197 (2005), no. 2, 554586 .

[Lev08] Adam Simon Levine, Computing knot Floer homology in cyclic branched covers, Algebr. Geom. Topol. 8 (2008), no. 2, 1163-1190.

[Lip06] Robert Lipshitz, A cylindrical reformulation of Heegaard Floer homology, Geom. Topol. 10 (2006), 955-1097, arXiv:math.SG/0502404.

[LL08] Dan Lee and Robert Lipshitz, Covering spaces and Q-gradings on Heegaard Floer homology, J. Symp. Geom. (2008), no. 1, 33-59.

[LOT08] Robert Lipshitz, Peter S. Ozsváth, and Dylan P. Thurston, Bordered Heegaard Floer homology: Invariance and pairing, 2008, arXiv:0810.0687v4.

[LOT11] Robert Lipshitz, Peter S. Ozsváth, and Dylan P. Thurston, Heegaard Floer homology as morphism spaces, Quantum Topol. 2 (2011), no. 4, 381-449, arXiv:1005.1248.

[LOT15] _ Bimodules in bordered Heegaard Floer homology, Geom. Topol. 19 (2015), no. 2, 525-724. 
[LT16] Robert Lipshitz and David Treumann, Noncommutative Hodge-to-de Rham spectral sequence and the Heegaard Floer homology of double covers, J. Eur. Math. Soc. (JEMS) 18 (2016), no. 2, 281-325.

[Lur09] Jacob Lurie, Higher topos theory, Annals of Mathematics Studies, vol. 170, Princeton University Press, Princeton, NJ, 2009.

[Man06] Ciprian Manolescu, Nilpotent slices, Hilbert schemes, and the Jones polynomial, Duke Math. J. 132 (2006), no. 2, 311-369.

[McC01] John McCleary, A user's guide to spectral sequences, second ed., Cambridge Studies in Advanced Mathematics, vol. 58, Cambridge University Press, Cambridge, 2001.

[MO07] Ciprian Manolescu and Brendan Owens, A concordance invariant from the Floer homology of double branched covers, Int. Math. Res. Not. IMRN (2007), no. 20, Art. ID rnm077, 21.

[MOS09] Ciprian Manolescu, Peter Ozsváth, and Sucharit Sarkar, A combinatorial description of knot Floer homology, Ann. of Math. (2) 169 (2009), no. 2, 633-660, arXiv:math.GT/0607691.

[MS04] Dusa McDuff and Dietmar Salamon, J-holomorphic curves and symplectic topology, American Mathematical Society Colloquium Publications, vol. 52, American Mathematical Society, Providence, RI, 2004.

[OSz03a] Peter S. Ozsváth and Zoltán Szabó, Absolutely graded Floer homologies and intersection forms for four-manifolds with boundary, Adv. Math. 173 (2003), no. 2, 179-261, arXiv:math.SG/0110170.

[OSz03b] Knot Floer homology and the four-ball genus, Geom. Topol. 7 (2003), 615-639, arXiv: math.GT/0301149.

[OSz04a] _ Holomorphic disks and three-manifold invariants: properties and applications, Ann. of Math. (2) 159 (2004), no. 3, 1159-1245, arXiv:math.SG/0105202.

[OSz04b] _ Holomorphic disks and topological invariants for closed three-manifolds, Ann. of Math. (2) 159 (2004), no. 3, 1027-1158, arXiv:math.SG/0101206.

[OSz05] - On the Heegaard Floer homology of branched double-covers, Adv. Math. 194 (2005), no. 1, 1-33, arXiv:math.GT/0309170.

[OSz06] _ Holomorphic triangles and invariants for smooth four-manifolds, Adv. Math. 202 (2006), no. 2, 326-400, arXiv:math.SG/0110169.

[OSz08]__ Holomorphic disks, link invariants and the multi-variable Alexander polynomial, Algebr. Geom. Topol. 8 (2008), no. 2, 615-692, arXiv:math/0512286.

[Per08] Timothy Perutz, Hamiltonian handleslides for Heegaard Floer homology, Proceedings of Gökova Geometry-Topology Conference 2007, Gökova Geometry/Topology Conference (GGT), Gökova, 2008, pp. $15-35$.

[Poź99] Marcin Poźniak, Floer homology, Novikov rings and clean intersections, Northern California Symplectic Geometry Seminar, Amer. Math. Soc. Transl. Ser. 2, vol. 196, Amer. Math. Soc., Providence, RI, 1999, pp. 119-181.

[Ras03] Jacob Rasmussen, Floer homology and knot complements, Ph.D. thesis, Harvard University, Cambridge, MA, 2003, arXiv:math.GT/0306378.

[Ras10] Jacob Rasmussen, Khovanov homology and the slice genus, Invent. Math. 182 (2010), no. 2, 419-447.

[RT11] Heather M. Russell and Julianna S. Tymoczko, Springer representations on the Khovanov Springer varieties, Math. Proc. Cambridge Philos. Soc. 151 (2011), no. 1, 59-81.

[Sei08] Paul Seidel, Fukaya categories and Picard-Lefschetz theory, Zurich Lectures in Advanced Mathematics, European Mathematical Society (EMS), Zürich, 2008.

[Sei15a] , The equivariant pair-of-pants product in fixed point Floer cohomology, Geom. Funct. Anal. 25 (2015), no. 3, 942-1007.

[Sei15b] _ Homological mirror symmetry for the quartic surface, Mem. Amer. Math. Soc. 236 (2015), no. 1116, vi+129.

[SS06] Paul Seidel and Ivan Smith, A link invariant from the symplectic geometry of nilpotent slices, Duke Math. J. 134 (2006), no. 3, 453-514.

[SS10] , Localization for involutions in Floer cohomology, Geom. Funct. Anal. 20 (2010), no. 6, 1464-1501.

[SSS14] Sucharit Sarkar, Cotton Seed, and Zoltán Szabó, A perturbation of the geometric spectral sequence in khovanov homology, 2014, arXiv:1410.2877. 
[SW10] Sucharit Sarkar and Jiajun Wang, An algorithm for computing some Heegaard Floer homologies, Ann. of Math. (2) 171 (2010), no. 2, 1213-1236, arXiv:math/0607777.

[Sza15] Zoltán Szabó, A geometric spectral sequence in Khovanov homology, J. Topol. 8 (2015), no. 4, 1017-1044.

[Twe14] Eamonn Tweedy, On the anti-diagonal filtration for the Heegaard Floer chain complex of a branched double-cover, J. Symplectic Geom. 12 (2014), no. 2, 313-363.

[Vog73] Rainer M. Vogt, Homotopy limits and colimits, Math. Z. 134 (1973), 11-52.

[Wal09] Jack W. Waldron, An invariant of link cobordisms from symplectic Khovanov homology, 2009, arXiv:0912.5067.

[Wat10] Liam Watson, A remark on Khovanov homology and two-fold branched covers, Pacific J. Math. 245 (2010), no. 2, 373-380.

[Weh09] Stephan M. Wehrli, A remark on the topology of $(n, n)$ Springer varieties, 2009, arXiv:0908.2185.

[Wu15] Weiwei Wu, Equivariant split generation and mirror symmetry of special isogenous tori, 2015, arXiv:1501.06257.

[WW12] Katrin Wehrheim and Chris T. Woodward, Floer cohomology and geometric composition of Lagrangian correspondences, Adv. Math. 230 (2012), no. 1, 177-228.

Mathematics Department, University of California, Los Angeles, CA 90095

E-mail address: hendricks@math.ucla.edu

Department of Mathematics, University of Oregon, Eugene, OR 97403

E-mail address: lipshitz@uoregon.edu

Department of Mathematics, University of California, Los Angeles, CA 90095

E-mail address: sucharit@math.ucla.edu 